\title{
QUANTUM ASPECTS OF ROAMING DYNAMICS
}

\author{
A Dissertation presented to \\ the Doctoral Committee of Casey D. Foley \\ at the University of Missouri-Columbia \\ Department of Chemistry
}

\author{
In Partial Fulfillment \\ of the Requirements for the Degree \\ Doctor of Philosophy
}

\author{
by \\ CASEY D. FOLEY \\ Dr. Arthur G. Suits, Dissertation Supervisor \\ DECEMBER 2020
}


(C) Copyright by Casey D. Foley 2020

All Rights Reserved 
The undersigned, appointed by the dean of the Graduate School, have examined the dissertation entitled

\title{
QUANTUM ASPECTS OF ROAMING DYNAMICS
}

\author{
presented by Casey D. Foley, \\ a candidate for the degree of Doctor of Philosophy of Chemistry, \\ and hereby certify that, in their opinion, it is worthy of acceptance.
}

Professor Arthur G. Suits

Professor David J. Robertson

Professor Kurt R. Brorsen

Professor Carsten A. Ullrich 
This dissertation is dedicated to my loving wife and parents who always believed in what I could accomplish despite my faults. 


\section{ACKNOWLEDGEMENTS}

I would like to thank my research advisor, Prof. Arthur Suits, for accepting me as a PhD student at a time when my future was uncertain. After spending almost three years performing analytical mass spectrometry experiments under the direction of a different research advisor, I made the tough choice to end my PhD candidacy at Wayne State University and nearly joined the workforce. Instead, I drove from Detroit, MI to Columbia, MO to meet Professor Suits and learn about his research. He welcomed me into his research group and made it an easy choice to continue my academic life at the University of Missouri. Prof. Suits has been on the cutting edge of chemical dynamics for decades and has taught me to think creatively, make hay while the sun is shining in the lab, and showed me countless tricks of the trade an experimental physical chemist needs. I can only hope that I carry some of his wisdom as my career progresses.

Prof. Bernadette Broderick guided me early on, where I did my best to assist in chirped pulsed uniform flow experiments, and she got me acquainted with chemical dynamics. Chandika Amarasinghe taught me how to fix and align lasers issues and how to use the Beta machine and where I collected all the data presented in this dissertation. He and Chaya Weeraratna also informed me of a potential opportunity to work in the Suits lab. James Thompson taught me how to use MATLAB and some key details of chemical dynamics. Greg Hall, master of many things including LabVIEW, provided a thorough tutorial on how to build LabVIEW programs and challenged many conclusions that Prof. Suits and I arrived at as we amassed formaldehyde data. Baptiste Joalland performed all the multireference calculations in this dissertation and explained to me the value of different methods. Tahereh Alavi also assisted with propargyl chloride calculations. Hongwei $\mathrm{Li}$ was beyond helpful in many areas including laser alignment, data analysis, and teaching me concepts of chemical dynamics. Graham Cooper, beyond being a friend to enjoy Mizzou and Kansas City football games with, also taught me concepts of chemical dynamics and PGOPHER basics, was essential to helping interpret HDCO PHOFEX spectra. Prof. Hua Guo and Prof. Changjian Xie were indispensable to interpreting the formaldehyde dynamics we were observing and put up with the many different calculations we asked them to perform. Jianzhuo Tu in the lab of Prof. Michael Harmata performed the paraformaldehyde-d1 synthesis that would have otherwise likely taken me a year to get done. 
Rod Schlotzhauer and Cheston Callais from machine shop at Mizzou were able to quickly fabricate different necessary for different experiments I performed on the Beta machine. Jerry Brightwell and Holly Oswald assisted me as I met the different milestones towards obtaining my Ph.D., whether it was ordering materials for my experiments, emailing me reminders of approaching deadlines, or countless other things. Holly also took care of my cats whenever I was out of town. Prof. Timothy Glass welcomed me into the Mizzou Chemistry Dept. and allowed for an easy transition during a stressful time. Finally, I would like to thank my dissertation committee members Prof. J. David Robertson, Prof. Kurt R. Brorsen, and Prof. Carsten A. Ullrich for their time and cooperation during the challenging year that is 2020 amidst the SARS-CoV-2 pandemic. The work presented in this thesis was supported by the Army Research Office and the National Science Foundation. 


\section{TABLE OF CONTENTS}

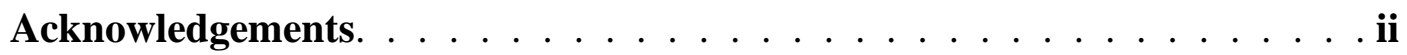

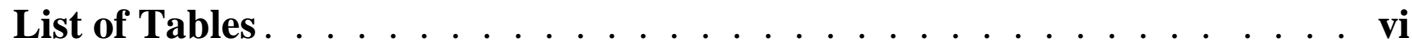

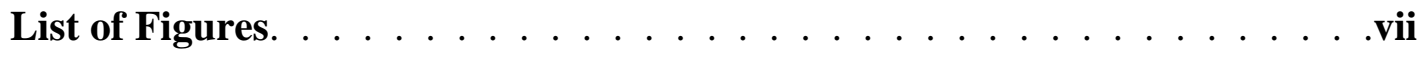

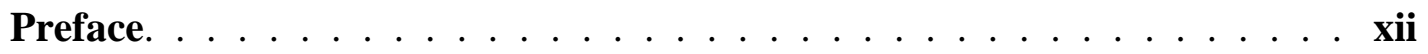

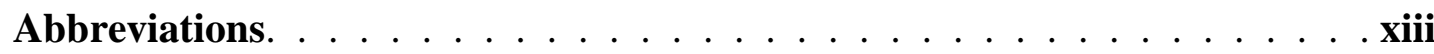

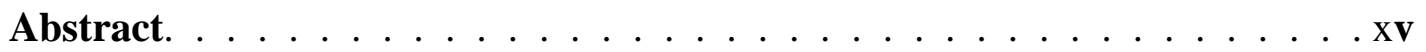

Chapter 1 -Introduction $\ldots \ldots \ldots \ldots \ldots \ldots \ldots$

1.1 Introduction. . . . . . . . . . . . . . . 1

1.2 Roaming Dynamics. . . . . . . . . . . . . . . 4

Chapter 2 - Experimental Methods . . . . . . . . . . . . . . . . . 14

2.1 Molecular Beam. . . . . . . . . . . . . . . . . . 14

2.2 Resonance-Enhanced Multiphoton Ionization. . . . . . . 15

2.3 Photofragment Imaging. . . . . . . . . . . . . . . 19

2.4 Instrument Setup. . . . . . . . . . . . . . . . . . 21

Chapter 3 - Mixed Transitions in the UV Photodissociation of Propargyl Chloride . . . . . . . . . . . . . . . 25

3.1 Introduction. . . . . . . . . . . . . . . . . . 25

3.2 Experimental Methods. . . . . . . . . . . . . . 28

3.3 Results and Discussion. . . . . . . . . . . . . . . . 32

3.4 Conclusion. . . . . . . . . . . . . . . 43

Chapter 4 - Infrared Multiphoton Excitation and Dissociation of Propargyl Chloride . . . . . . . . . . . . . . . . 46

4.1 Introduction. . . . . . . . . . . . . . . . 46

4.2 Experimental Methods. . . . . . . . . . . . . . . . . .48

4.3 Results and Discussion. . . . . . . . . . . . . . . . 49 
4.4 Conclusion. . . . . . . . . . . . . . . . . . . . 63

\section{Chapter 5 - HDCO Spectroscopy} and Radical Dissociation Thresholds. . . . . . . . . . . . . 64

5.1 Introduction. . . . . . . . . . . . . . . . . . . 64

5.2 Experimental Methods. . . . . . . . . . . . . . . . . 67

5.3 Results and Discussion . . . . . . . . . . . . . . . . 69

5.4 Conclusion. . . . . . . . . . . . . . . . . . .

Chapter 6 - Quantum Aspects of the Roaming Pathway in Formaldehyde Photodissociation . . . . . . . . . . . . 77

6.1 Introduction. . . . . . . . . . . . . . . . . . . . .77

6.2 Experimental Methods. . . . . . . . . . . . . . . . . . .78

6.3 Results . . . . . . . . . . . . . . . . . . . . . . 79

6.4 Discussion . . . . . . . . . . . . . . . 91

6.5 Conclusion. . . . . . . . . . . . . . . . . . . 101

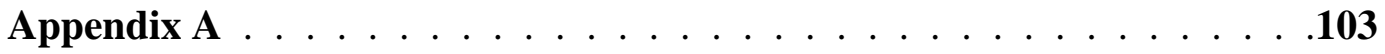

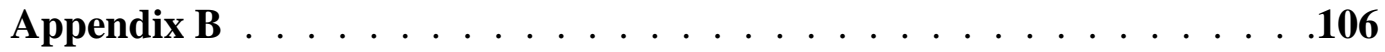

Appendix C . . . . . . . . . . . . . . . . . . . . . .119

Appendix D . . . . . . . . . . . . . . . . . . . . . 157

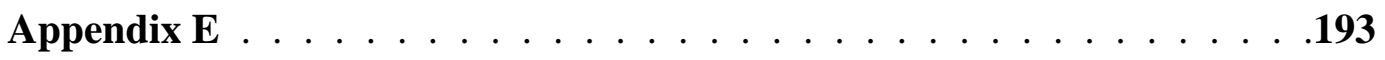

Bibliography . . . . . . . . . . . . . . . . 196

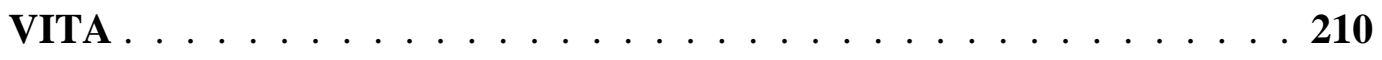




\section{LIST OF TABLES}

Table 2.1 REMPI schemes used in every chapter summarized.

Table 3.1 Summary of theoretical calculations. Excitation energies from the ground state singlet to the first 5 singlet and 6 triplet states calculated with various active spaces and multireference methods at the optimized $\operatorname{CCSD}(\mathrm{T})$ singlet ground state geometry. Oscillator strengths of the singlet-singlet transitions estimated from SA6-CASSCF(10/9) transition dipole moments and SA12-MRCI-F12+Q energies. All calculations were done with the aug $(\mathrm{Cl})$-cc-pVTZ basis set. Energies are expressed in $\mathrm{eV}$.

Table 3.2 Energy partitioning of the $\mathrm{Cl} / \mathrm{Cl}^{*}$ photofragments at indicated nominal wavelength. Precise excitation energies are given in methods. The $0 \mathrm{~K}$ dissociation energy, $68.6 \mathrm{kcal} \mathrm{mol}^{-1}$, is obtained from ref. 46. $\mathrm{E}_{\mathrm{tot}}$ and $\mathrm{Ea}_{\mathrm{vl}}$ stand for excitation energy and available energy after dissociation on the ground state, respectively, $\mathrm{E}_{\mathrm{T}}$ for the translational energy of each $\mathrm{Cl} / \mathrm{Cl}^{*}$ peak, $\mathrm{E}_{\text {int }}, \mathrm{E}_{\mathrm{rot}}$, and $\mathrm{E}_{\text {vib }}$ for the internal, rotational, and vibrational energies of $\mathrm{C}_{3} \mathrm{H}_{3}$ according to the impulsive model (see text for details). fvib and $\mathrm{f}_{\mathrm{T}}$ are the fractions of available energy appearing in $\mathrm{C}_{3} \mathrm{H}_{3}$ vibrational and total translational energies, respectively. Energies are expressed in $\mathrm{kcal} \mathrm{mol}^{-1}$.

Table 6.1 Rotational lines of $2^{1} 4^{3}$ and their energies. 


\section{LIST OF FIGURES}

Figure 1.1 Schematic illustration of $\mathrm{S}_{0}$ dissociation pathways of formaldehyde. Adapted with permission from A.G. Suits, Ann. Rev. Phys. Chem., 71, 77-100 (2020) (C) Annual Reviews.

Figure 1.2 Left. Ion images of $\mathrm{j}_{\mathrm{CO}}=40(\mathrm{~A}), \mathrm{j}_{\mathrm{CO}}=28(\mathrm{~B})$, and $\mathrm{j}_{\mathrm{CO}}=15(\mathrm{C})$. Right. Solid lines are experimental translational energy distributions of the corresponding images and dashed lines are determined by quasi-classical trajectories. Combs indicate the $\mathrm{H}_{2}$ vibrational state. Adapted with permission from D. Townsend et al, Science, 306, 1158-1161 (2004) (C) AAAS.

Figure 1.3 The roaming atom transition state, $\mathrm{TS}_{\mathrm{R}}(\mathrm{A})$. The tight transition state, $\mathrm{TS}_{\mathrm{T}}$ leading to molecular products. Potential energy curves resulting from linear interpolation. Adapted with permission from L.B. Harding et al, Phys. Chem. Chem. Phys., 9, 4055-4070 (2007) (C) the Owner Societies 2007 and L.B. Harding et al, J. Phys. Chem. A, 116, 6967-6982 (2012) @ ACS.

Figure 1.4 PHOFEX spectra of different jco used to approximate the roaming onset. Adapted with permission from S.A. Lahankar et al, Chem. Phys., 347, 288-299 (2008) (C) Elsevier.

Figure 1.5 Roaming channel branching fractions from CO ( $\mathrm{v}=0)$ from Lahankar, Sutis, and coworkers (red dots), Kable and coworkers (black dots), and a quasiclassical trajectory study. Adapted with permission from M.S. Quinn et al, J. Chem. Phys., 147, 013935 (2017) (C) AIP.

Figure 2.1 Simulated SIMION expansion for repeller voltage of $2500 \mathrm{~V}$ with three electrodes (A), $500 \mathrm{~V}$ with a single electrode (B), and $500 \mathrm{~V}$ with four electrodes. Adapted with permission from D. Townsend et al, Rev. Sci. Instrum., 74(4), 2530-2539 (2003) (C) AIP.

Figure 2.2 Images of $\mathrm{O}\left({ }^{3} \mathrm{P}_{2}\right)$ that are $\mathrm{DC}$ sliced (A), unsliced (B), and unsliced and reconstructed. Adapted with permission from D. Townsend et al, Rev. Sci. Instrum., 74(4), 2530-2539 (2003) (C) AIP.

Figure 2.3 Schematic of the ion lens assembly containing the four electrodes repeller, extractor $\left(\mathrm{L}_{1}\right)$, focusing lens $1\left(\mathrm{~L}_{2}\right)$, and focusing lens $2\left(\mathrm{~L}_{3}\right)$. Units are in mm. Adapted with permission from D. Townsend et al, Rev. Sci. Instrum., 74(4), 2530-2539 (2003) (C) AIP.

Figure 2.4 Schematic of the experimental setup with a cut-through of the ion optics assembly. Adapted with permission from D. Townsend et al, Rev. Sci. Instrum., 74(4), 25302539 (2003) (C) AIP. 
Figure 3.1 Structures of the ${ }^{1} \mathrm{~A}^{\prime}$ and ${ }^{3} \mathrm{~A}$ minima optimized at the $\operatorname{CCSD}(\mathrm{T})$ level, along with singlet-triplet separation energy $\triangle \mathrm{E}$ including $\mathrm{ZPE}$ correction.

Figure 3.2 DC slice images of $\mathrm{Cl}(\mathrm{A})$ and $\mathrm{Cl}^{*}(\mathrm{~B})$ from photodissociation of propargyl chloride near $236 \mathrm{~nm}$. Laser polarization axis is vertical.

Figure 3.3 Translational energy distributions (black) and anisotropy parameter $\beta$ (blue) of $\mathrm{Cl}$ (A) and $\mathrm{Cl}^{*}$ (B) from photodissociation of propargyl chloride near $236 \mathrm{~nm}$.

Figure 3.4 DC slice images of $\mathrm{Cl}(\mathrm{A}) \mathrm{Cl}^{*}$ (B) and $\mathrm{C}_{3} \mathrm{H}_{3}$ (C) from photodissociation of propargyl chloride near $212 \mathrm{~nm}$. Laser polarization axis is vertical.

Figure 3.5 Translational energy distributions (black) and anisotropy parameter $\beta$ (blue) of $\mathrm{Cl}$ (A) $\mathrm{Cl}^{*}(\mathrm{~B})$ and $\mathrm{C}_{3} \mathrm{H}_{3}(\mathrm{C})$ from photodissociation of propargyl chloride near 212 $\mathrm{nm}$.

Figure 3.6 Absorption spectrum of propargyl chloride (left, blue) at $295 \mathrm{~K}$ (adapted from Fahr et al.42) and excitation energies of the singlet and triplet states located below $8 \mathrm{eV}$ (3 $\mathrm{x}^{1} A^{\prime}$ (black), $3 \times{ }^{1} A^{\prime \prime}$ (blue), $3 \mathrm{x}^{3} A^{\prime}$ (red), $3 \mathrm{x}^{3} A^{\prime \prime}$ (purple), ) calculated at the MRCI-F12/aug(Cl)-cc-pVTZ level. Laser excitation wavelengths are shown with black arrows and a tentative assignment of the features observed in the experimental spectrum with dashed lines. Note that strong vibronic couplings are expected for excitations to the $\mathrm{S} 1, \mathrm{~S} 2, \mathrm{~S} 3$, and $\mathrm{S} 4$ states due in part to the presence of double-wells along the low-frequency CCC bending coordinate (see Fig. 3.7).

Figure 3.7 Potential energies of the ground state $\mathrm{S}_{0}$ and the five first singlet excited states calculated at the SA6-CASSCF(10/9)/cc-pVTZ level along the $\mathrm{q}_{14}$ normal mode.

Figure 3.8 Potential energy cuts along the $\mathrm{RC}-\mathrm{Cl}$ coordinate leading to the $\mathrm{C} 3 \mathrm{H} 3+\mathrm{Cl}$ and $\mathrm{C}_{3} \mathrm{H}_{3} *+\mathrm{Cl}$ asymptotes. The level of theory is SA12- CASSCF(10/11)/cc-pVTZ and the geometry is relaxed on the ground state by optimization at the MP2/ccpVTZ level. The color code for state symmetry and multiplicity is the same as in Fig. 5. The molecular structure corresponds to a minimum on the $2{ }^{3} A^{\prime \prime}$ state, which correlates to the $\mathrm{C} 3 \mathrm{H} 3 *+\mathrm{Cl}$ asymptote and could play a role as an intermediate before the dissociation occurs towards the ground state.

Figure 4.1 DC slice images of 1-color UV Cl (A), 2-color $\mathrm{Cl}(\mathrm{B}), 1$-color $\mathrm{UV} \mathrm{Cl}^{*}(\mathrm{C})$, and 2color $\mathrm{Cl}^{*}$ (D) from photodissociation of propargyl chloride. Laser polarization axis is vertical in the plane of the figure. 
Figure 4.2 Translational energy distributions of $\mathrm{Cl}(\mathrm{A})$ and $\mathrm{Cl}^{*}$ (B) from 1-color UV (black) and 2-color (red) photodissociation of propargyl chloride photodissociation.

Figure 4.3 Translational energy distributions (black) and anisotropy parameter $\beta$ (blue) of $\mathrm{Cl}$ from 1-color UV (A) and 2-color (B) photodissociation of propargyl chloride.

Figure 4.4 Translational energy distributions (black) and anisotropy parameter $\beta$ (blue) of $\mathrm{Cl}^{*}$ from 1-color UV (A) and 2-color (B) experimental configurations of propargyl chloride photodissociation.

Figure 4.5 Experimental HCl IRMPD REMPI spectrum (top trace) and simulation at $85 \mathrm{~K}$ (bottom trace).

Figure 4.6 DC slice image of $\mathrm{HCl}(\mathrm{v}=0, \mathrm{j}=1)(\mathrm{A})$ and translational energy distribution (black) and anisotropy parameter $\mathrm{b}$ (blue) (B) from 2-color photodissociation of propargyl chloride. Red lines show decomposition of the distribution to two components (see text).

Figure 4.7 Relative energies (in $\mathrm{kcal} \mathrm{mol}^{-1}$ ) of stationary points on the ground state singlet potential energy surface of $\mathrm{C}_{3} \mathrm{H}_{3} \mathrm{Cl}$, including zero-point energy correction. The roaming TS is encircled in red. Values are from CBS-QB3 calculations and, in parentheses, from wB97XD/aug-cc-pVDZ calculations.

Figure 4.8 CPUF rotational spectra for indicated $\mathrm{C}_{3} \mathrm{H}_{2}$ isomers following $193 \mathrm{~nm}$ photodissociation of 1,2-butadiene and propargyl chloride (A). Branching to $1-\mathrm{C}_{3} \mathrm{H}_{2}$ for propargyl chloride (blue solid line) or 1,2-butadiene (red dashed line) (B).

Figure 5.1 Schematic of relevant PES cuts with energies and pathways indicated. Adapted with permission from M.S. Quinn et al, J. Chem. Phys., 147, 013935 (2017) @ AIP.

Figure 5.2 Left. Inertial axis designations. Right. Normal modes of vibration and symmetry species. Adapted with permission from D.J. Clouthier et al, Ann. Rev. Phys. Chem., 34, 31-58 (1983) (C) Annual Reviews and D.E. Reisner et al, J. Chem. Phys., 80(12), 5968-5978 (1983) C AIP.

Figure 5.3 HDCO PHOFEX spectra with jco $=44$ REMPI detection. Green dots indicate transitions excited in imaging experiments. Inset: expanded view of select vibrational bands.

Figure 5.4 Selected images of $\mathrm{H}$ atom (left column) and D atom (right column) fragments from excitation on four different rovibrational lines of HDCO. 
Figure 5.5 Translational energy plotted against upper state energy for $\mathrm{H}$ atom (red) and D atom (blue) fragments.

Figure 5.6 D atom translational energy distributions of three selected images displayed in Figure 5.3.

Figure 5.7 $\mathrm{H}$ atom translational energy distributions of three selected images displayed in Figure 5.3.

Figure 6.1 $2^{1} 4^{3}$ PHOFEX spectrum with $\mathrm{j}_{\mathrm{CO}}=44$ REMPI detection (top) and PGOPHER simulation (bottom). Commonly used ortho rotational lines and the four para rotational lines are labeled.

Figure 6.2 Rotational distributions for $\mathrm{CO}(\mathrm{v}=0)$ following excitation of a range of parent $\mathrm{H}_{2} \mathrm{CO}$ rotational levels on the $2^{1} 4^{3}$ band. All have been normalized to the same total population.

Figure 6.3 DC slice images of $\mathrm{j}_{\mathrm{CO}}=28(\mathrm{~A}), \mathrm{j}_{\mathrm{CO}}=37(\mathrm{~B})$ and $\mathrm{j}_{\mathrm{CO}}=45(\mathrm{~B})$ for a series of ortho and para excitations of $2^{1} 4^{3}$.

Figure 6.4 Total translational energy distributions of a series jco images for a series of ortho and para excitations of $2^{1} 4^{3}$.

Figure 6.5 DC slice images of a series of $2^{1} 6^{1}$ para excitations of $\mathrm{j}_{\mathrm{CO}}=23(\mathrm{~A})$ and $\mathrm{j}_{\mathrm{CO}}=45$ (B). Associated total translational energy distributions of $\mathrm{j}_{\mathrm{CO}}=23$ (C) and $\mathrm{j}_{\mathrm{CO}}=45$ (D).

Figure 6.6 Raw CO rotational distributions for several ortho and para excitations on the $2^{1} 4^{1}$ band.

Figure 6.7 DC slice images of $\mathrm{j}_{\mathrm{CO}}=23$ dissociated from $2^{2} 4^{1}(\mathrm{~A}), \mathrm{j}_{\mathrm{CO}}=45$ dissociated from $2^{2} 4^{1}(B), j_{C O}=23$ dissociated from $2^{2} 4^{3}(C), j_{C O}=45$ dissociated from $2^{2} 4^{3}$ (D).

Figure 6.8 Associated total translational energy distributions for the images in Fig. 6.7 for a series of ortho and para excitations of $2^{2} 4^{3}$ (left) and $2^{2} 4^{3}$ (right).

Figure 6.9 Roaming fraction (orange) relative to the overall CO rotational distribution (purple) for $3_{03}$ (top) and $2_{02}$ (bottom) on $2^{1} 4^{3}$.

Figure 6.10 Symmetry coupling "blocks" describing how an initially prepared state on $\mathrm{S}_{1}$ couples to $\mathrm{S}_{0}$. 
Figure 6.11 Energy ladder of $\mathrm{H}_{2} \mathrm{CO}$ (left) and $\mathrm{H}+\mathrm{HCO}$ (right) rotational states. The states involved in a resonance are indicated by the red box. Adapted with permission from A.C. Terentis et al, J. Chem. Phys., 108 (8), 3187-3198 (1998) (C) AIP.

Figure 6.12 Relative energy vs roaming fractions for ortho (red circles) and para transitions (blue diamonds). The region where a resonance between $\mathrm{H}_{2} \mathrm{CO}$ and $\mathrm{H}+\mathrm{HCO}$ states is observed is indicated by the blue box, with associated $\mathrm{CO}$ rotational distributions. Other ortho transitions (green box) and associated $\mathrm{CO}$ rotational distributions are also included.

Figure 6.13 Total translational energy distributions for $\mathrm{j} \mathrm{co}=23$ and 28 for excitations near the identified resonance.

Figure 6.14 In-plane restricted (red) vs. normal (blue) trajectory results.

Figure 6.15 Wavefunction near the radical threshold shown for dihedral angle of $78^{\circ}$. Tight $\mathrm{TS}$ and roaming saddle point structures are marked. 


\section{PREFACE}

This dissertation is based on the following refereed publications and soon to be published formaldehyde and HDCO results that will yield multiple publications. The publication listed for Chapter 6 is nearing completion and will be submitted by the end of 2020 .

Chapter 3 - C.D. Foley, B. Joalland, S.T. Alavi, A.G. Suits. Mixed Transitions in the UV Photodissociation of Propargyl Chloride Revealed by Slice Imaging and Multireference ab initio Calculations. Phys. Chem. Chem. Phys. 20: 27474-27481, 2018.

Chapter 4 - C.D. Foley, S.T. Alavi, B. Joalland, B.M. Broderick, N. Dias, A.G. Suits. Imaging the Infrared Multiphoton Excitation and Dissociation of Propargyl Chloride. Phys. Chem. Chem. Phys. 21: 1528-1535, 2018.

Chapter 5 - C.D. Foley, G.A. Cooper, J. Tu, M. Harmata, A.G. Suits. HDCO Radical Dissociation Thresholds by Velocity Map Imaging. Mol. Phys. DOI: 10.1080/00268976.2020.1813344, 2020.

Chapter 6 - C.D. Foley, C. Xie, H. Guo and A. G. Suits. Quantum Roaming Dynamics in $\mathrm{H}_{2} \mathrm{CO}$ at Threshold. in preparation. 


\section{ABBREVIATIONS}

\begin{tabular}{|c|c|}
\hline PES & potential energy surface \\
\hline RRKM & Rice-Ramsperger-Kassel-Marcus \\
\hline TST & transition state theory \\
\hline UV & ultraviolet \\
\hline IRMPE & infrared multiphoton excitation \\
\hline IRMPD & infrared multiphoton dissociation \\
\hline $\mathrm{TS}$ & transition state \\
\hline REMPI & resonance-enhanced multiphoton ionization \\
\hline $\mathrm{DC}$ & direct current \\
\hline $\mathrm{KE}$ & kinetic energy \\
\hline TED & translational energy distribution \\
\hline QCT & quasi-classical trajectory \\
\hline $\mathrm{S} / \mathrm{N}$ & signal-to-noise \\
\hline PTS & photofragment translational spectroscopy \\
\hline PMT & photomultiplier tube \\
\hline LIF & laser-induced fluorescence \\
\hline $\mathrm{MCP}$ & microchannel plate \\
\hline VMI & velocity map imaging \\
\hline $\mathrm{TOF}$ & time-of-flight \\
\hline Fin $A$ & finite slice analysis \\
\hline VUV & vacuum ultraviolet \\
\hline $\mathrm{ZPE}$ & zero-point energy \\
\hline $\mathrm{FC}$ & Franck-Condon \\
\hline CASSCF & complete active space self-consistent field \\
\hline
\end{tabular}


TEA CPUF transverse excitation atmospheric

chirped pulse quasi-uniform flow 


\begin{abstract}
Decades of experimental and theoretical achievements built on the foundations of thermochemistry and quantum mechanics have birthed the field of molecular dynamics where intimate details of individual reactive events can be mapped. On the ground state of formaldehyde, two dissociation pathways are commonly known, one proceeding over a high barrier through a three-center, tight transition state to give molecular products, and another via homolytic simple bond fission to radical products. A third, distinct ground state dissociation pathway first seen in formaldehyde 15 years ago, the 'roaming' mechanism, involves incipient radicals, but the $\mathrm{H}$ atom samples a large, flat region of the PES, roaming in the van der Waals region, leading to an intramolecular $\mathrm{H}+\mathrm{HCO}$ reaction. Roaming reactions have been visualized from a classical perspective with only a few exceptions in the literature despite the likely quantum nature of the process. A major objective of the presented work is to reveal quantum aspects of roaming reactions. Here, a few different molecules $\left(\mathrm{C}_{3} \mathrm{H}_{3} \mathrm{Cl}, \mathrm{HDCO}\right.$, and $\left.\mathrm{H}_{2} \mathrm{CO}\right)$ were investigated with the goal of characterizing roaming radicals on the ground state, where roaming observations have primarily occurred.
\end{abstract}

Knowledge of the excited states of propargyl chloride was necessary to understand the experimental observations from investigation of its ground state. Ultraviolet (UV) photodissociation and state-specific detection with velocity map imaging of $\mathrm{Cl}, \mathrm{Cl}^{*}$, and $\mathrm{C}_{3} \mathrm{H}_{3}$ were interpreted with the aid of multireference calculations to characterize the nature of the electronic excitations. A series of triplet states were identified to preferentially dissociate to $\mathrm{Cl}$ or $\mathrm{Cl}^{*}$. Infrared multiphoton excitation and infrared multiphoton dissociation (IRMPD) of propargyl chloride enhanced UV processes and allowed for radical dissociation at threshold. IRMPD on the 
ground state produced $\mathrm{HCl}$ following isomerization to 1-chloroallene, where a roaming-like transition state with $\mathrm{Cl}$ in an abstraction geometry is adopted.

Accurate $\mathrm{H}$ and $\mathrm{D}$ atom ground state radical thresholds of singly deuterated formaldehyde, HDCO, were obtained from velocity map imaging to aid future studies HDCO dynamics studies. The different radical thresholds, arising from the difference in zero-point energies, is a purely quantum phenomenon. PHOFEX spectra over a wide range were collected, creating a library of known frequencies of rotational lines that can be used to study the dynamics of different vibrational bands and the energy dependence of different processes. Heats of formation of HDCO and DCO were also determined.

Finally, a detailed examination of the photochemical dynamics in both ortho and para nuclear spin isomers of formaldehyde is provided, exploring the full range of parent rotational levels from $\mathrm{J}=0$ to 4 , initially prepared via excitation of specific rotational lines of a range of vibrational bands on the first singlet excited state $\left(2^{1} 4^{1}, 2^{1} 6^{1}, 2^{1} 4^{3}, 2^{2} 4^{1}, 2^{2} 4^{3}\right)$. Measurements of the entire $\mathrm{CO}(\mathrm{v}=0)$ product rotational distributions are combined with velocity map imaging of selected CO product states to obtain a complete picture of the photochemical dynamics for each specific parent rotational level. Distributions are found to vary dramatically with small changes in total energy, effects not captured at all by classical treatments. Full quantum state correlations reveal interactions among three formaldehyde dissociation continua, yielding rich insight into the dynamics of this highly excited molecule as it decays to products. An orbiting resonance dominates the dynamics, though other possible dynamical phenomena are noted. 


\section{CHAPTER 1 INTRODUCTION}

\subsection{Introduction}

The fundamental understanding of chemical reactions grew from the foundation of thermochemistry. Heats of formation of reactants and products are essential to inform where the energy in different steps of a reaction is partitioned, and databases are maintained to tabulate these values. ${ }^{1,2}$ Thermochemistry says nothing of the necessary steps to change from one substance to another. Quantum mechanics expanded the scientific pursuit to use molecular structure to understand reactivity and created detailed methods to interpret and predict spectra. Spectral databases $^{3-7}$ containing energies of different quantum states and transitions of thousands of molecules and powerful software ${ }^{8}$ now exist to aid in our spectral assignments and predictions. Decades of experimental and theoretical achievements built on these foundations has birthed the field of molecular dynamics where intimate details of individual reactive events can be mapped. Crucial technological developments such as molecular beams, high speed vacuum pumps, and high power density lasers with narrow linewidths for quantum state-resolved detection of atoms and molecules saw a proliferation of chemical dynamics experiments. Knowledge of the fundamental interactions of isolated reactions provides a panoramic view of the convoluted network of reactions that occur in astrochemical, atmospheric, and combustion processes. In the gas phase, isolated molecules and single reactive events, whether unimolecular dissociation or bimolecular collisions, can be investigated. Key concepts of elementary chemical reactions and processes have been developed through gas phase studies, culminating in the 1986 Nobel Prize in Chemistry being shared by Dudley R. Herschbach, Yuan T. Lee, and John C. Polanyi. ${ }^{9-11}$ 
These concepts learned from simple reactions remain broadly applicable to many different, more complex systems today. Furthermore, experiments studying simple reactions were essential in the development of robust computational methods that are used to build the potential energy surfaces (PES) that shape predictions of the possible pathways that are most likely to occur in a reaction. Electronic structure methods continue to improve and can completely describe ground and excited states, and how they are coupled. Stationary point structures on the energy landscape can be accurately determined and are essential to predicting rate constants in unimolecular processes as a function of energy by the Rice-Ramsperger-Kassel-Marcus (RRKM) expression used in transition state theory (TST) of chemical reactivity. ${ }^{12-16}$ In the RRKM expression, the transition state barrier is defined as the highest point on the minimum energy path between reactants and products. ${ }^{17}$ Numerous systems, however, have been identified experimentally and theoretically that do not follow the minimum energy path and constitute a significant portion of the reactive flux. Variation in the unimolecular rate constant with energy and the magnitude of the rate constant in van der Waals molecules, for example, have been known to disagree with RRKM theory. ${ }^{18-20}$ These pathways that stray from the typical, minimum energy pathway have come to be known as 'roaming' pathways.

Roaming reactions have been visualized from a classical perspective with only a few exceptions in the literature. ${ }^{21,22}$ Quasi-classical, ${ }^{23-27}$ statistical, ${ }^{28-31}$ and TST phase space perspectives $^{32-35}$ have primarily been used to treat the roaming mechanism despite the extreme quantum nature of the process, which will be described in detail in the next section. Experiments focused on uncovering the quantum aspects that affect the dynamics have also not been conducted apart from theoretical investigations of $\mathrm{H}+\mathrm{MgH} \rightarrow \mathrm{Mg}+\mathrm{H}_{2} \cdot{ }^{21,22,36}$ Most roaming systems are considered too large or energetic for a quantum mechanical description, and there are a many 
dynamical possibilities that could possibly obscure the determination of a quantum effect. Although $\mathrm{NO}_{3}$ showed the importance of roaming in excited electronic states, ${ }^{37-39}$ roaming observations have primarily occurred on the ground state, and in this thesis experiments were performed on a few different molecules with the goal of investigating roaming radicals on the ground state. A major objective of the present work is to reveal quantum aspects of roaming reactions.

Complex excited dynamics of propargyl chloride were observed while preparing for investigation of its ground state dynamics. ${ }^{40}$ Knowledge of the excited states of propargyl chloride are necessary to interpret the experimental observations from investigation of its ground state, and provide rich insight into the electronic excitations of a substituted $s p$ hybridized system. ${ }^{41}$ Thus, ultraviolet (UV) photodissociation of propargyl chloride is first covered in Chapter 3 before moving on to infrared multiphoton excitation (IRMPE) and infrared multiphoton dissociation (IRMPD) of propargyl chloride in Chapter 4. Experiments on singly deuterated formaldehyde, $\mathrm{HDCO}$, are then presented in Chapter 5 where $\mathrm{H}$ and $\mathrm{D}$ atom radical thresholds are determined to aid future studies that will probe the effect of symmetry on formaldehyde dynamics. ${ }^{42}$ This difference, arising from the difference in zero-point energies, is a purely quantum phenomenon, although a familiar one. Finally, the most revelatory results, quantum aspects of $\mathrm{H}_{2} \mathrm{CO}$ photodissociation dynamics, are discussed in Chapter 6. Because the roaming mechanism is vital to understanding the impact of the presented results, an overview of the phenomenon is first provided. 


\subsection{Roaming Dynamics}

Though formaldehyde photodissociation dynamics and spectroscopy will be covered in greater detail in Chapters 5 and 6, the most instructive and well-studied example of roaming is that of formaldehyde. Selected roaming investigations of formaldehyde are thus used to explain the mechanism. A characterization of the roaming mechanism was first provided through joint theoretical and experimental studies of $\mathrm{H}_{2} \mathrm{CO} \cdot{ }^{24,43,44}$ Earlier studies had reported related dynamical behavior, though without painting a full picture of the underlying dynamics or identifying the mechanism causing the observables. ${ }^{45-50}$

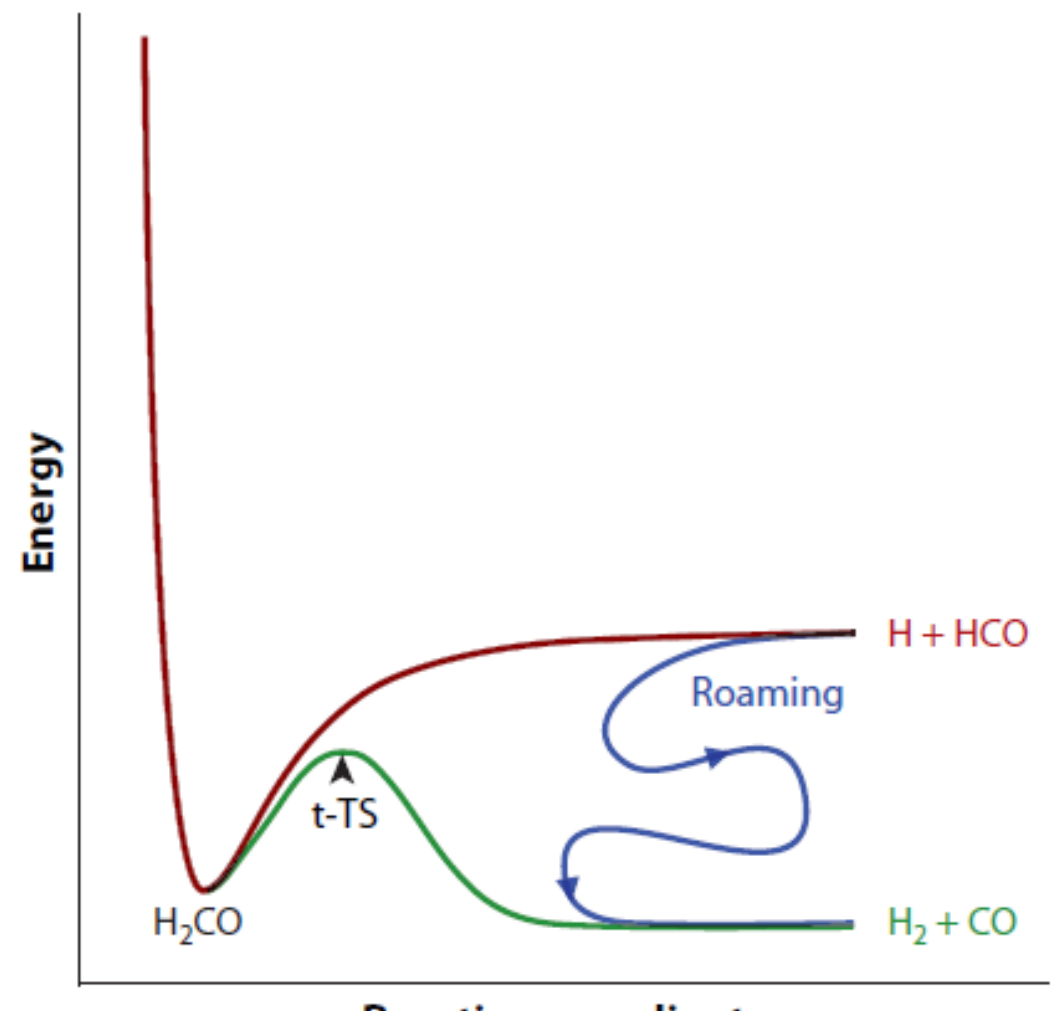

\section{Reaction coordinate}

Fig. 1.1 Schematic illustration of $S_{0}$ dissociation pathways of formaldehyde. Adapted with permission from A.G. Suits, Ann. Rev. Phys. Chem., 71, 77-100 (2020) (C) Annual Reviews.

On the ground state of formaldehyde, dissociation by a third way was identified and described in detail. ${ }^{24}$ The commonly known pathway to molecular fragments, Equation 1.1, proceeds over a high barrier through a three-center, tight transition state (TS) (Figure 1.1, green 
line). The other established pathway to radical fragments by homolytic simple bond fission, Eq. 1.2, opens at higher energy (Fig.1.1, red line). The third, distinct pathway involves incipient radicals, but the $\mathrm{H}$ atom samples a large, flat region of the PES, roaming in the van der Waals region, leading to an intramolecular $\mathrm{H}+\mathrm{HCO}$ reaction (Fig. 1.3, blue line), Eq. 1.3.

$$
\begin{gathered}
\mathrm{H}_{2} \mathrm{CO} \rightarrow \mathrm{H}_{2}+\mathrm{CO} \\
\mathrm{H}_{2} \mathrm{CO} \rightarrow \mathrm{H}+\mathrm{HCO} \\
\mathrm{H}_{2} \mathrm{CO} \rightarrow[\mathrm{H}--\mathrm{HCO}] \rightarrow \mathrm{H}_{2}+\mathrm{CO}
\end{gathered}
$$

Evidence of an alternate pathway to molecular products in formaldehyde dissociation was first observed by Moore and coworkers. ${ }^{51}$ When energies above the radical threshold were used to excite formaldehyde, the $\mathrm{CO}$ rotational distribution had a larger low-jco population, with $j_{\mathrm{co}}$ the CO rotational quantum number. Two explanations were offered, one stated that the anharmonicity of the transition state at higher energies allows for additional geometries to be sampled and produce $\mathrm{CO}$ with lower rotational excitation. The other explanation was the presence of a distinct pathway to molecular products related to opening of the radical channel and a subsequent intramolecular hydrogen abstraction.

More than a decade later, high resolution photofragment imaging of vibrationless $\mathrm{CO}$ rotational states from $\mathrm{H}_{2} \mathrm{CO}$ photodissociation was performed to resolve the question of the dynamics involved. ${ }^{24}$ Formaldehyde was excited with a single photon from a ground state, $\mathrm{S}_{0}$, of an initial rovibrational state energy, $\mathrm{E}_{\mathrm{S} 0}$, to a rovibrational state of the first singlet excited state, $\mathrm{S}_{1}$, at a precise energy, $\mathrm{E}_{\mathrm{S} 1}$, above the radical threshold. The energy of the photon, $h v$, was,

$$
h v=E_{S I}-E_{S 0}
$$


Internal conversion to the ground state, $S_{0}$, produces vibrationally excited formaldehyde that subsequently dissociates via the pathways described in Eqs. 1.1 to 1.3. Specific CO rotational states were probed via resonance-enhanced multiphoton ionization (REMPI), and by using weak direct current (DC) velocity mapping focusing fields the recoiling products were imaged. Velocity distributions were thus visualized for different $\mathrm{CO}$ rotational states, with smaller to larger radii structure corresponding to increasing velocity of the detected $\mathrm{CO}$ fragment.

For the dissociation $\mathrm{H}_{2} \mathrm{CO} \rightarrow \mathrm{H}_{2}+\mathrm{CO}$, products recoil at a distribution of velocities. If the velocity of $\mathrm{CO}$ can be measured, the total translational energy $\left(\mathrm{E}_{\mathrm{T}(\mathrm{CO})}\right)$ of $\mathrm{CO}$ can be calculated by Eq. 1.4 using the mass of $\mathrm{CO}$ and its detected velocity.

$$
E_{T(C O)}=\frac{1}{2} m_{C O} v_{C O}^{2}
$$

Conservation of momentum dictates that,

$$
m_{\mathrm{CO}} v_{\mathrm{CO}}+m_{\mathrm{H} 2} v_{\mathrm{H} 2}=0
$$

The total translational energy release $\left(\mathrm{E}_{\mathrm{T}(\mathrm{total})}\right)$ from the dissociation event contained in $\mathrm{CO}$ and $\mathrm{H}_{2}$ can thus be determined:

$$
\begin{gathered}
E_{T(\text { total })}=E_{T(\mathrm{CO})}+E_{T(\mathrm{H} 2)} \\
=E_{T(\mathrm{CO})}+\frac{1}{2} m_{\mathrm{H} 2} v_{H 2}{ }^{2} \\
=E_{T(\mathrm{CO})}+\frac{1}{2} m_{\mathrm{H} 2}\left(\frac{m_{\mathrm{CO}} v_{C O}}{m_{H 2}}\right)^{2} \\
=E_{T(\mathrm{CO})}+\left(\frac{m_{C O}}{m_{H 2}}\right)\left(\frac{1}{2} m_{C O} v_{C O}^{2}\right)
\end{gathered}
$$




$$
\begin{aligned}
& =E_{T(C O)}+E_{T(C O)} \frac{m_{C O}}{m_{H 2}} \\
& =E_{T(C O)} \frac{m_{C O}+m_{H 2}}{m_{H 2}} \\
& =E_{T(C O)} \frac{m_{H 2 C O}}{m_{H 2}}
\end{aligned}
$$

The available energy in the system is:

$$
E_{\text {available }}=E_{S I}-\Delta H_{f}
$$

where $\Delta \mathrm{H}_{\mathrm{f}}$ is the difference in the $0 \mathrm{~K}$ heats of formation of the reactant, $\mathrm{H}_{2} \mathrm{CO}$, and products, $\mathrm{H}_{2}$ $+\mathrm{CO}$. The available energy must be distributed amongst the degrees of freedom of $\mathrm{H}_{2}$ and $\mathrm{CO}$, or

$$
E_{\text {total }}=E_{T(\text { total })}+E_{I N T, H 2}+E_{I N T, C O}
$$

where $\mathrm{E}_{\mathrm{INT}, \mathrm{H} 2}$ and $\mathrm{E}_{\mathrm{INT}, \mathrm{CO}}$ are the internal energies of $\mathrm{H}_{2}$ and $\mathrm{CO}$, respectively.

State-resolved detection via REMPI allows for the internal energy of CO to be known exactly by virtue of the detected quantum state, and the energy in the system can thus be tracked. Only the internal energy of $\mathrm{H}_{2}$ is unknown, and ground state $\mathrm{H}_{2}$ rovibrational levels have well defined energies. Smaller to larger radii structure in $\mathrm{CO}$ photofragment images thus correspond to decreasing internal energy of the $\mathrm{H}_{2}$ cofragment. Rotational states of $\mathrm{j}_{\mathrm{CO}}=40,28$ and 15 were imaged (Fig. 1.2, left) and the translational energy distributions (TEDs) were extracted from the images (Fig. 1.2, right). For $\mathrm{j}_{\mathrm{CO}}=40,28$ and $15, \mathrm{H}_{2}$ vibrational states from $\mathrm{v}=0$ to $3, \mathrm{v}=0$ to 7 in a bimodal distribution, and v $=5$ to 7 were formed, respectively. Quasi-classical trajectories (QCT) were performed from two different geometries, one at the global $\mathrm{S}_{0}$ minimum and the other with one $\mathrm{CH}$ bond initially stretched to twice the equilibrium length. TEDs produced by these 
trajectories were able to qualitatively match the experimental data (Fig. 1.2, right, dashed lines) and confirm the existence of the roaming mechanism.
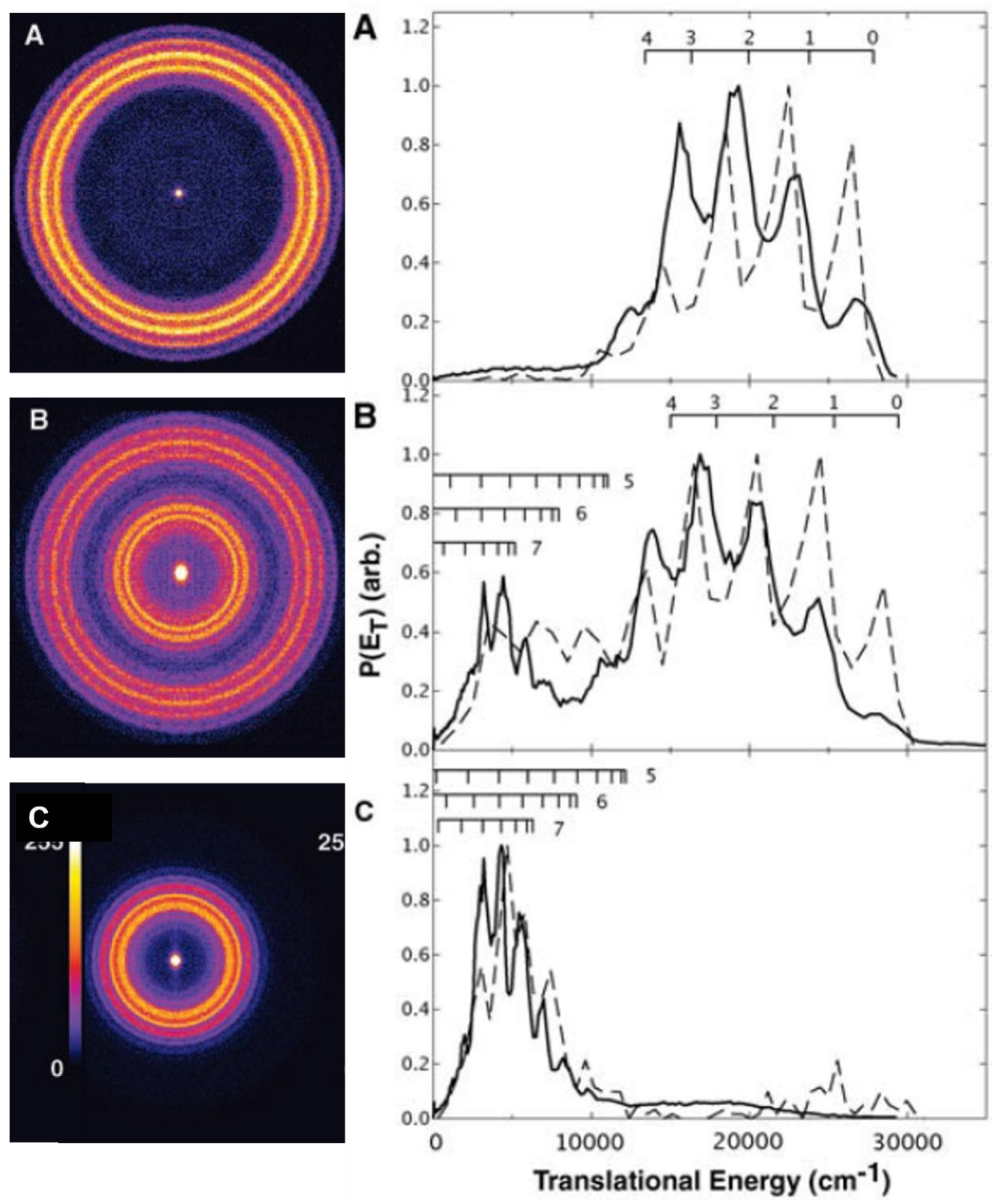

Fig. 1.2 Left. Ion images of $\mathrm{j}_{\mathrm{CO}}=40(\mathrm{~A}), \mathrm{j}_{\mathrm{CO}}=28(\mathrm{~B})$, and $\mathrm{j}_{\mathrm{CO}}=15(\mathrm{C})$. Right. Solid lines are experimental translational energy distributions of the corresponding images and dashed lines are determined by quasi-classical trajectories. Combs indicate the $\mathrm{H}_{2}$ vibrational state. Adapted with permission from D. Townsend et al, Science, 306, 1158-1161 (2004) (C) AAAS.

Key aspects of TST are challenged by roaming reactions. In TST, reactants and products are associated with distinct regions of configuration space and are necessarily separated by an $n-1$ dimensional hypersurface, where $n$ are the internal degrees of freedom of the system. Any pathway 
connecting reactants to products must pass through this dividing surface. A variationally optimized dividing surface is referred to as the variational transition state. Though first-order saddle points and transition states are fundamentally different geometric objects, the transition state dividing surface often contains a first-order saddle point and sometimes these are referred to interchangeably. Soon after roaming was first reported, Klippenstein and coworkers identified a roaming saddle point (Fig. 1.3A) with an imaginary frequency of $140 \mathrm{~cm}^{-1}$ and two lowest bound frequencies of only $20 \mathrm{~cm}^{-1}$ and $80 \mathrm{~cm}^{-1} \cdot{ }^{30}$ Roaming saddle points have been useful in identifying

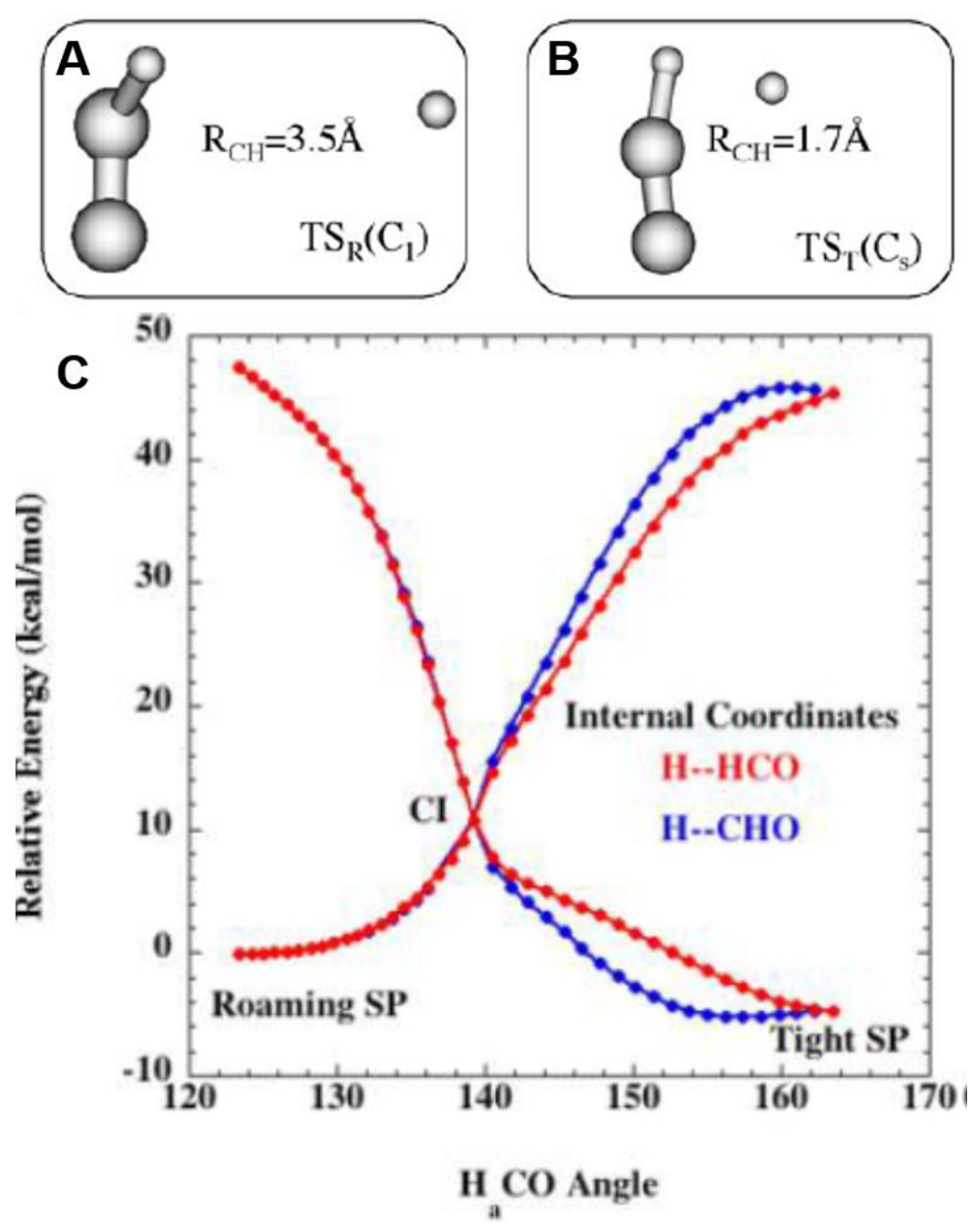

Fig. 1.3 The roaming atom transition state, $\mathrm{TS}_{\mathrm{R}}(\mathrm{A})$. The tight transition state, $\mathrm{TS}_{\mathrm{T}}$ leading to molecular products. Potential energy curves resulting from linear interpolation. Adapted with permission from L.B. Harding et al, Phys. Chem. Chem. Phys., 9, 4055-4070 (2007) (C) the Owner Societies 2007 and L.B. Harding et al, J. Phys. Chem. A, 116, 6967-6982 (2012) @ ACS. 
roaming dynamics in many other systems, but their relevance remains controversial. Because these saddle points are in flat, anharmonic regions of the PES, TST assumptions of harmonic frequencies and no dynamical recrossing are invalidated, making determination of accurate rate coefficients difficult to impossible. Furthermore, roaming product flux may not necessarily come close to the configuration of the saddle point. The relation of roaming saddle points to the typical tight saddle point $^{30}$ (Fig. 1.3B) and the effect on the underlying dynamics is also uncertain, though a dividing surface connecting these saddle points in formaldehyde have likely pass through a conical intersection. (Fig. 1.3C) ${ }^{29}$ The separability of these two regions suggested that the pathways are energetically separable, distinct pathways from a kinetic point of view, but we will visit this question in detail in Chapter 6. ${ }^{29}$

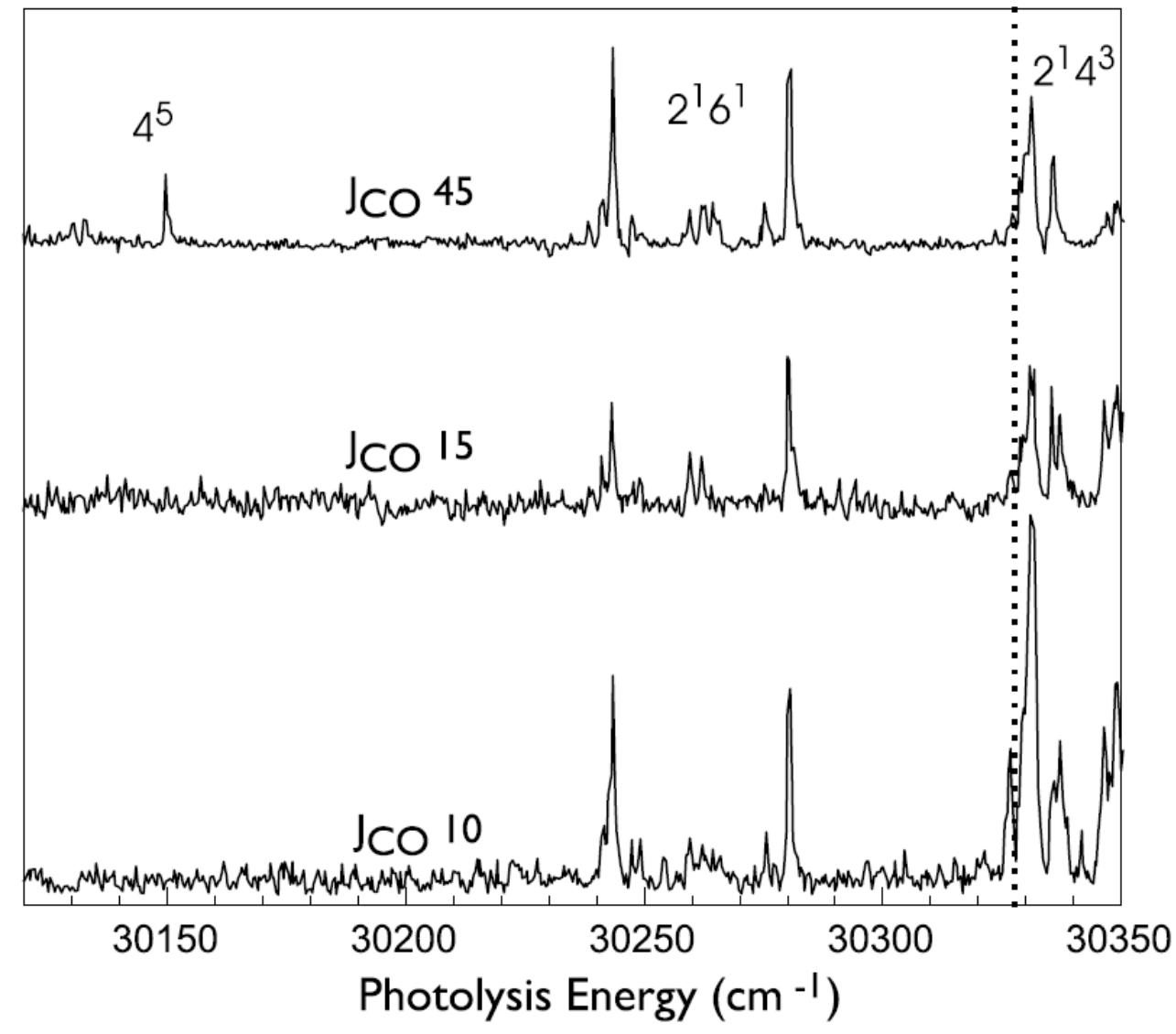

Fig. 1.4 PHOFEX spectra of different jco used to approximate the roaming onset. Adapted with permission from S.A. Lahankar et al, Chem. Phys., 347, 288299 (2008) () Elsevier. 
Following the initial investigations of formaldehyde, there were questions regarding the energy at which the roaming channel opened and the energy dependence of roaming branching. Suits and coworkers collected photofragment excitation (PHOFEX) spectra to determine approximately the energy of the roaming onset (Fig. 1.4). ${ }^{52}$ By fixing a probe laser to detect a low jco state, exclusively produced by roaming, and scanning the pump laser from low to high energy, the first appearance of a peak indicates that the roaming threshold is at least as low as that pump energy. Because formaldehyde is excited at specific, resonant energies corresponding to welldefined rotational lines, it is possible that the roaming onset would be lower than the first detected peak. The roaming onset was determined to be at least $80 \mathrm{~cm}^{-1}$ below the radical threshold. ${ }^{52}$

An energy dependence study by Lahankar et al. spanning more than $1200 \mathrm{~cm}^{-1}$ indicated an increasing roaming fraction with energy. ${ }^{25}$ An increased propensity to roam was concluded by observing a larger population of vibrationally excited $\mathrm{H}_{2}$ cofragments in a $j_{\mathrm{CO}}=28$ image and an increasing population of $j_{\mathrm{CO}}=15$ but relatively uniform $j_{\mathrm{CO}}=45$ population with energy in a PHOFEX scan. The CO rotational distribution, however, obtained from the commonly used excitation energy of $\sim 30340 \mathrm{~cm}^{-1}$ was assumed to be invariant. Full CO rotational distributions over a broader range of energy, $\sim 6000 \mathrm{~cm}^{-1}$, were later collected by Kable and coworkers that showed the rotational distribution from the tight TS pathway markedly increased with energy while that from the roaming pathway stayed relatively the same. ${ }^{53}$ The fraction of $\mathrm{H}_{2}$ and $\mathrm{CO}$ from roaming was determined to be relatively constant at $\sim 20 \%$ over the entire energy range (Fig. 1.5). However, these measurements were made for a single rotational level on each vibrational band, and we will see this is not adequate to capture the full picture. A comparison of experimental results from the Lahankar and Kable and papers is included in Fig. 1.5 in addition to results from QCT recently performed by Houston and coworkers. ${ }^{54}$ The results to be presented in Chapter 6, 
however, show that profound quantum effects are manifested in $j_{\mathrm{CO}}$ images and $\mathrm{CO}$ rotational distributions at the radical dissociation threshold and fade with increasing energy.

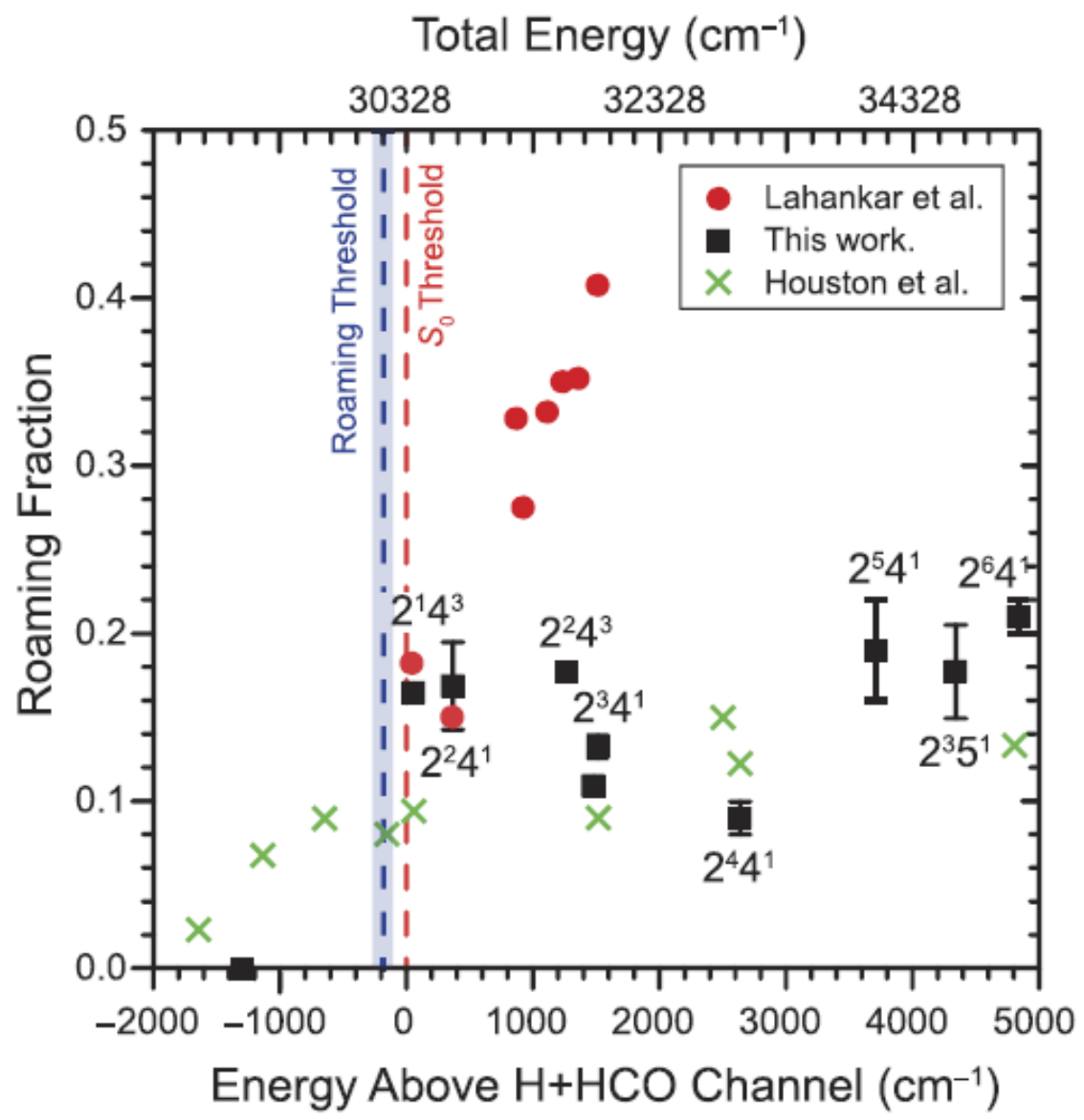

Fig. 1.5 Roaming channel branching fractions from $\mathrm{CO}(\mathrm{v}=0)$ from Lahankar, Sutis, and coworkers (red dots), Kable and coworkers (black dots), and a quasiclassical trajectory study. Adapted with permission from M.S. Quinn et al, J. Chem. Phys., 147, 013935 (2017) (C) AIP.

Roaming is now viewed as a universal aspect of chemical reactivity with numerous theoretical and experimental investigations revealing the phenomenon in different classes of compounds and environments. ${ }^{44}$ For example, roaming has been identified in aldehydes, ${ }^{24,55,56}$ ketones, ${ }^{57}$ alkanes, ${ }^{28}$ and nitro compounds ${ }^{26,37-39,58-60}$ and processes such as hydrocarbon combustion, ${ }^{61-65}$ atmospheric chemistry, ${ }^{66,67}$ and isomerization. ${ }^{26,59,68,69}$ Here, the library of compounds that roam is expanded to included propargyl chloride, ${ }^{41}$ and quantum aspects of roaming are described for the first time through a new approach to studying formaldehyde 
photodissociation dynamics. Before illustrating these newly discovered dynamics of propargyl chloride and formaldehyde, a description of the experimental methods is provided. 


\section{CHAPTER 2 \\ EXPERIMENTAL METHODS}

\subsection{Molecular Beam}

Essential to these experiments is a population of parent molecules that are confined to a small, well-defined number of quantum states. An ensemble of molecules spread over a large range of initial states permits more rotational and vibrational lines to be excited, creating congested spectra and poorer signal-to-noise $(\mathrm{S} / \mathrm{N})$ of the states that are desired to be studied. A medium that does not introduce changes to the sample or congest molecular spectra, an issue in matrix isolation experiments, for example, is necessary. A low temperature ensemble of molecules in a collisionfree environment is also required to prevent thermal decomposition and intermolecular processes. A molecular beam created by a supersonic expansion of pressurized gas into high vacuum is utilized to create these experimental conditions.

Molecular rotations are cooled to temperatures well below the freezing point of the molecule in a supersonic expansion. ${ }^{70-76}$ The expansion is isentropic, converting random thermal motion into directed mass flow. The translational temperature is defined by the width of the velocity distribution, which narrows in the expansion. The overall flow velocity increases while the local speed of sound decreases, creating a flow above Mach number 1, or supersonic. Early in the expansion when density is still sufficiently high, collisions between molecules cool vibrational (to some extent) and rotational degrees of freedom that remain populated. Equilibration between translation and rotational degrees of freedom is more efficient than between translation and vibrational degrees of freedom, though molecules are mostly in $\mathrm{v}=0$ in a room temperature Boltzmann distribution. Molecules are often diluted in a noble gas such as He or Ar because they do not store energy in rotational or vibrational degrees of freedom, they give narrow velocity 
distributions and higher peak intensities, and to reduce cluster formation of the parent molecule of interest.

For an isentropic expansion, the isentropic equation relates temperature and pressure,

$$
\frac{T}{T_{0}}={\frac{P}{P_{0}}}^{(\gamma-1) / \gamma}
$$

where $\mathrm{T}$ and $\mathrm{T}_{0}$ are final and initial temperatures, $\mathrm{P}$ and $\mathrm{P}_{0}$ are final and initial pressures, and $\gamma$ is the specific heat ratio $\mathrm{C}_{\mathrm{p}} / \mathrm{C}_{\mathrm{v}}$. A greater pressure difference between the pressurized reservoir and the region the gas will expand into creates a lower molecular beam temperature.

For the experiments in this dissertation, gas phase molecules of interest were contained in a reservoir between 1000 and 1700 Torr at a concentration of $\sim 5 \%$ unless specifically stated. The reservoir pressurized a valve, in this case a pulsed solenoid General valve made by Parker Hannifin. The source chamber the molecular beam expands into is maintained at $\sim 10^{-6}$ torr. While the valve is pulsing, the source chamber pressure increases by two orders of magnitude. The result is a molecular beam with a parent molecule with a rotational temperature that is below $25 \mathrm{~K}$.

\subsection{Photofragment Imaging}

The famous Stern-Gerlach experiment is likely the first beam imaging ever performed. Attempting to illustrate space quantization of orbital angular momentum, a collimated beam of silver atoms was passed through a magnetic field, where the beam consequently split and was imaged on a glass plate. ${ }^{77,78}$ Spin was detected, not orbital angular momentum, but it did present a new way to study photochemical processes. Photoabsorption experiments of $\mathrm{CH}_{3}$ and $\mathrm{CD}_{3}$ using photographic plates are another of the earliest examples of imaging chemical dynamics. ${ }^{79}$ Photolysis mapping employed a glass hemisphere with a tellurium film reacted with 
photofragment radicals and enabled the first detection of previously predicted anisotropic photofragment angular distributions..$^{80,81}$

Photofragment translational spectroscopy (PTS) subsequently became widespread using the universal detection method electron impact ionization. PTS involved detecting the anisotropy of angular distributions from bimolecular collisions or unimolecular dissociation by recording mass spectra at different angles with a rotatable detector and sometimes rotatable molecular beam sources. ${ }^{82-89}$ PTS and Doppler probe ${ }^{90,91}$ methods were the methods of choice used in photodissociation dynamics studies for decades. Other methods were also developed that paved the way for photofragment imaging such as a state-specific imaging with an 80 photomultiplier tube (PMT) array using laser-induced fluorescence (LIF), ${ }^{92}$ and a more commonly used technique by Los and coworkers that could map pairs of photofragments in time and position on a microchannel plate (MCP). ${ }^{93-98}$

Chandler and Houston developed a method to map the dissociation products spatially on a MCP coupled to a phosphor screen. ${ }^{99}$ Methyl iodide was photodissociated, methyl and iodine fragments were allowed to separate, traveling at a particular recoil velocity, and the methyl fragment was ionized state-specifically with a laser and extracted by a grounded mesh screen after the repeller ion lens. The location of the ions was detected on the MCP/phosphor and imaged with a polaroid film. Ground state $\mathrm{CH}_{3}$, and excited umbrella mode and $\mathrm{CH}$ stretch components were discerned, and vibrational level depended branching of I and I* were determined. ${ }^{100}$ The method offered state-specific detection, a leap in collection efficiency, and a simpler setup.

Eppink and Parker improved the experimental setup by removing extractor grids and obstructive ion optics that blurred images and limited velocity resolution. ${ }^{101}$ The focusing 
conditions of their ion optics improved velocity resolution by an order magnitude, resulting in the first demonstration of velocity map imaging (VMI). Kitsopoulos later introduced time slicing, where ion optics are turned on a short time after dissociation and ionization, ${ }^{102}$ allowing for the ion cloud to expand along the time-of-flight (TOF), causing a spread in arrival time. Utilizing nanosecond gating of the $\mathrm{MCP} /$ phosphor allowed for only the central slice of the 3D velocity distribution to be imaged, where previously the entire 3D velocity distribution had been imaged as a projection on the 2D detector. The method, unfortunately, sacrificed VMI focusing due to the necessity of grids to achieve uniform acceleration of ions and ion density had to be kept low to overcome space charge effects. The ability to image only the central slice of the 3D ion cloud and its corresponding velocity distribution should not be understated. Unsliced images introduce noise, reduce resolution, and require reconstruction methods that require cylindrical symmetry that may not be compatible with certain studies such vector correlations. Another generation of VMI, DC slice imaging, overcame these shortcomings by reducing the electric field in the initial acceleration region and adding an additional ion lens for refocusing. ${ }^{103}$ The results described here were all performed on the original DC slice imaging machine, and the experimental geometry of the apparatus and imaging details are described.

When a molecule is photodissociated, its photoproducts may be subsequently ionized with a focused probe laser. Upon photodissociation with unpolarized photons, photoproducts expand with recoil velocities in all directions, a 3D expansion known as a Newton sphere. Ionization of photoproducts immediately after dissociation between the repeller and extractor allows for a sphere of ions to be expanded temporally, while allowing their velocity to be spatially mapped on the position sensitive $\mathrm{MCP} / \mathrm{phosphor}$ detector. Because fragments travel in all directions, an accurate TED is obtained by measuring the central slice of the Newton sphere, which only contains 
photofragments that were initially recoiling parallel to the plane of the detector. The reduced repeller voltage and additional focusing lens of the DC slice configuration allows for a significantly expanded TOF and ion cloud size (Fig. 2.1). Using SIMION 7.0, expansion of TOF width from 40 to $190 \mathrm{~ns}$ was demonstrated for ${ }^{35} \mathrm{Cl}$ with $1 \mathrm{eV}$ translation energy, simply by reducing the repeller voltage from $2500 \mathrm{~V}$ to $500 \mathrm{~V}$ (Figs. 2.1A and 2.1B). The width of the newton sphere itself expands further to fill a larger portion of the detector, resulting in higher resolution. The addition of another focusing lens expands the TOF to $400 \mathrm{~ns}$ (2.1C). A comparison of $\mathrm{O}\left({ }^{3} \mathrm{P}_{2}\right)$ images from $\mathrm{NO}_{2}$ photodissociation illustrates the advantage of DC slicing (Fig. 2.2). Multiple rings that can inform the underlying dissociation dynamics are clear for the sliced image (Fig. 2.2A), but are obscured in the unsliced image (Fig. 2.2B), and only become resolved upon image reconstruction, which produces a line of noise about the symmetry axis (2.2C).

The angular distributions of the rings in images often possess varying degrees of anisotropy, as in Fig. 2.2. The images in Fig. 2.2 are anisotropic, due to the use of vertically polarized light that is parallel to the plane of the detector. The anisotropy of a component of a given velocity distribution is determined by extracting the intensity of a particular radius as a function of $\theta$, the angle relative to the polarization vector of the dissociation laser. Laser light in all experiments in this dissertation, except IR photons, was vertically polarized, parallel to the plane of the detector. For a one photon dissociation, angular distributions are obtained by fitting an image at each radius to the well-known expression ${ }^{104,105}$

$$
I(\theta) \propto 1+\beta\left(P_{2}(\cos \theta)\right)
$$

where $\mathrm{P}_{2}$ is the second Legendre polynomial, $\frac{1}{2}\left(3 \cos ^{2} \theta-1\right)$. The anisotropy parameter $\beta$ characterizes the photofragment angular distribution, ranging from -1 ("perpendicular") to +2 
("parallel") for the case of linear photolysis polarization. If a photofragment angular distribution is isotropic with $\beta \approx 0$ this does not necessarily imply a dissociation that is both parallel and perpendicular, but is most likely indicative of a slow dissociation process where molecular orientation had time to be scrambled prior to detection.
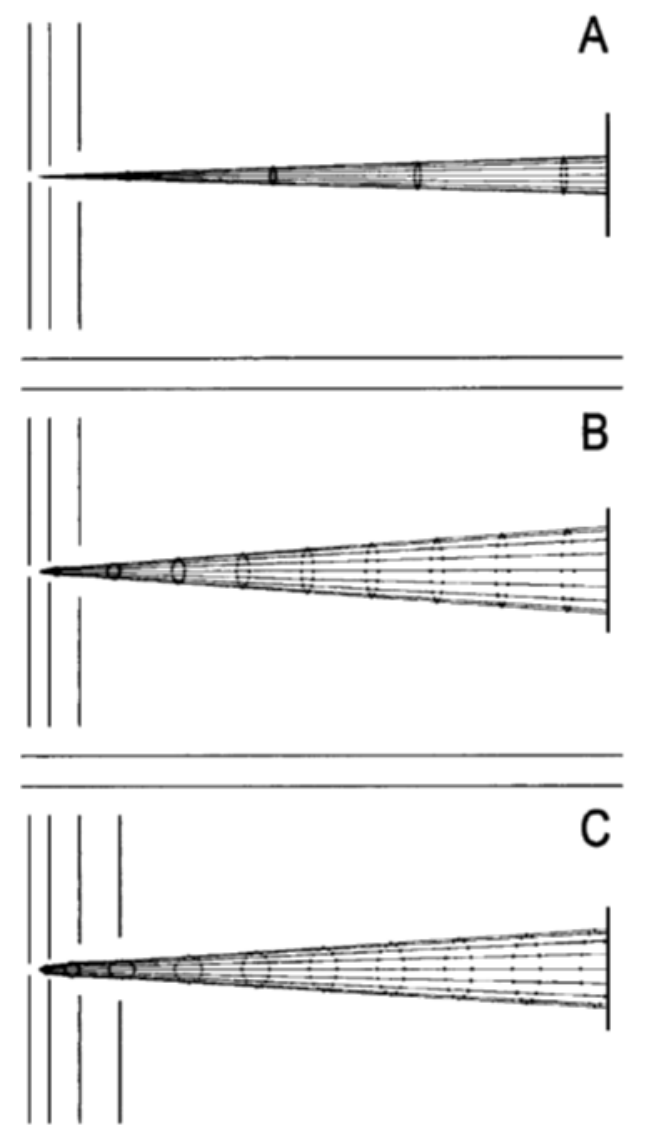

Fig. 2.1 Simulated SIMION expansion for repeller voltage of $2500 \mathrm{~V}$ with three electrodes (A), $500 \mathrm{~V}$ with a single electrode (B), and $500 \mathrm{~V}$ with four electrodes. Adapted with permission from D. Townsend et al, Rev. Sci. Instrum., 74(4), 2530-2539 (2003) (C AIP.

\subsection{Resonance-Enhanced Multiphoton Ionization}

Quantum state-specific detection of atoms and molecules is a valuable for detailed investigation of photodissociation dynamics, and essential for revealing quantum aspects of roaming dynamics. REMPI is a technique that allows for state-specific detection, and when polarized laser beams are employed can reveal photofragment angular distributions when coupled 
with VMI. REMPI was initially used to study electronic spectroscopy of atoms and molecules, where photon energies resonant with electronic states lead to an increase in ion intensity due to an increase in ionization efficiency when scanning the wavelength of a tunable laser.

A

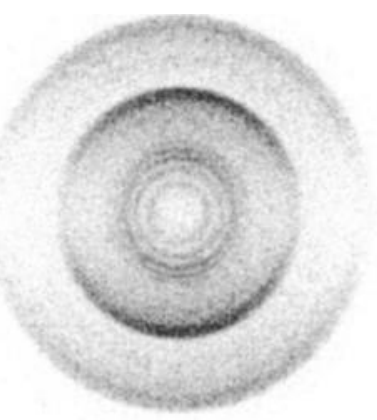

B

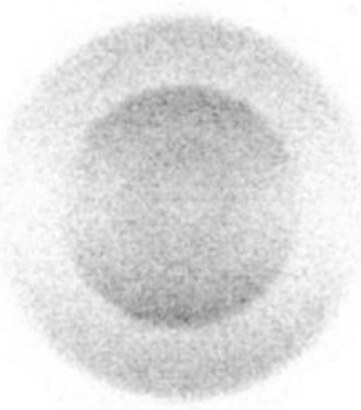

C

Fig. 2.2 Images of $\mathrm{O}\left({ }^{3} \mathrm{P}_{2}\right)$ that are DC sliced (A), unsliced (B), and unsliced and reconstructed. Adapted with permission from D. Townsend et al, Rev. Sci. Instrum., 74(4), 2530-2539 (2003) (C) AIP.

REMPI detection schemes are referred to as $(m+n)$, where $m$ is the number of resonant photons to excite an atom or molecule from a ground to an exited state and $\mathrm{n}$ is the number required to take the energy past the ionization continuum. For example, here $\mathrm{CO}$ photofragments dissociated from formaldehyde are detected via $(2+1)$ REMPI by a B $\left({ }^{1} \Sigma^{+} \mathrm{g}\right) \leftarrow \mathrm{X}\left({ }^{1} \Sigma^{+} \mathrm{g}\right)$ excitation using the $\mathrm{Q}$ branch of the transition. ${ }^{106}$ REMPI detection was also used for $\mathrm{Cl}^{3}{ }^{3} \mathrm{Cl}^{*},{ }^{3} \mathrm{C}_{3} \mathrm{H}_{3}$, 
$\mathrm{HCl},{ }^{107,108}$ and $\mathrm{H}$ atom. ${ }^{3}$ Table 2.1 summarizes the REMPI schemes used in each chapter. Although the velocity of a neutral photofragment following ionization remains largely unchanged because nearly all the excess energy in the ionization process is carried by the light electron, the limiting factor in velocity resolution is often due to non-negligible blurring of the velocity distribution from ionization. ${ }^{109,110}$

Table 2.1 REMPI schemes used to ionize photofragments

\begin{tabular}{ccccc}
\hline Species & Transition & Scheme & $\begin{array}{c}\text { Photon Energy } \\
\left(\mathrm{cm}^{-1}\right)\end{array}$ & Chapters \\
\hline $\mathrm{Cl}$ & ${ }^{2} \mathrm{D}^{\circ}{ }_{3 / 2} \leftarrow{ }^{2} \mathrm{P}^{\circ}{ }_{3 / 2}$ & $2+1$ & 42494.2 & 3,4 \\
$\mathrm{Cl}$ & ${ }^{2} \mathrm{P}^{\circ}{ }_{3 / 2} \leftarrow{ }^{2} \mathrm{P}^{\circ}{ }_{3 / 2}$ & $1+1$ & 47157.1 & 3 \\
$\mathrm{Cl}^{*}$ & ${ }^{2} \mathrm{P}_{3 / 2}^{\circ} \leftarrow{ }^{2} \mathrm{P}^{\circ}{ }_{1 / 2}$ & $2+1$ & 42280.0 & 3 \\
$\mathrm{Cl}^{*}$ & ${ }^{2} \mathrm{P}_{3 / 2}^{\circ} \leftarrow{ }^{2} \mathrm{P}^{\circ}{ }_{1 / 2}$ & $1+1$ & 46715.9 & 3 \\
$\mathrm{C}_{3} \mathrm{H}_{3}$ & $3{ }^{2} \mathrm{~B}_{2} \leftarrow 1{ }^{2} \mathrm{~B}_{2}$ & $1+1$ & $\sim 47170$ & 3 \\
$\mathrm{Cl}^{*}$ & ${ }^{2} \mathrm{P}_{3 / 2}^{\circ} \leftarrow{ }^{2} \mathrm{P}^{\circ}{ }_{1 / 2}$ & $2+1$ & 42721.22 & 4 \\
$\mathrm{HCl}$ & $\mathrm{F}^{1} \Delta_{2} \leftarrow \leftarrow \mathrm{X}^{1} \Sigma^{+}$ & $2+1$ & 41890.1 & 4 \\
$\mathrm{H}$ & $2 \mathrm{~s}\left({ }^{2} \mathrm{~S}_{1 / 2}\right) \leftarrow 1 \mathrm{~s}\left({ }^{2} \mathrm{~S}_{1 / 2}\right)$ & $2+1$ & 41129.6 & 5,6 \\
$\mathrm{CO}$ & $\mathrm{B}\left({ }^{1} \Sigma_{\mathrm{g}}^{+}\right) \leftarrow \mathrm{X}\left({ }^{1} \Sigma_{\mathrm{g}}^{+}\right)$ & $2+1$ & $\sim 43480$ & 5,6 \\
\hline
\end{tabular}

\subsection{Instrument Setup}

The machine used in these experiments is composed of many essential components and has previously been described in detail. ${ }^{103}$ The primary sections of the machine include the source chamber, main chamber, TOF tube, and detector, with each containing multiple important parts. The source chamber is pumped by a Varian V 300HT turbomolecular pump. The main chamber is pumped by an Osaka TG1113MBW-09 magnetically levitated turbomolecular pump. The source 
chamber is maintained at $10^{-6}$ Torr and contains a General valve with a skimmer cone mounted in front it on the aluminum plate separating the source from the main chamber. The skimmer cone has $1 \mathrm{~mm}$ aperture and samples the central part of supersonic expansion, resulting in a cool, collimated beam entering the main chamber, which is maintained at $3 \times 10^{-8}$ Torr. While the valve is pulsing, the source chamber pressure increases by two orders of magnitude and the main chamber increases by an order of magnitude. The main chamber has the four electrode DC slice imaging ion optics (Fig. 2.3).

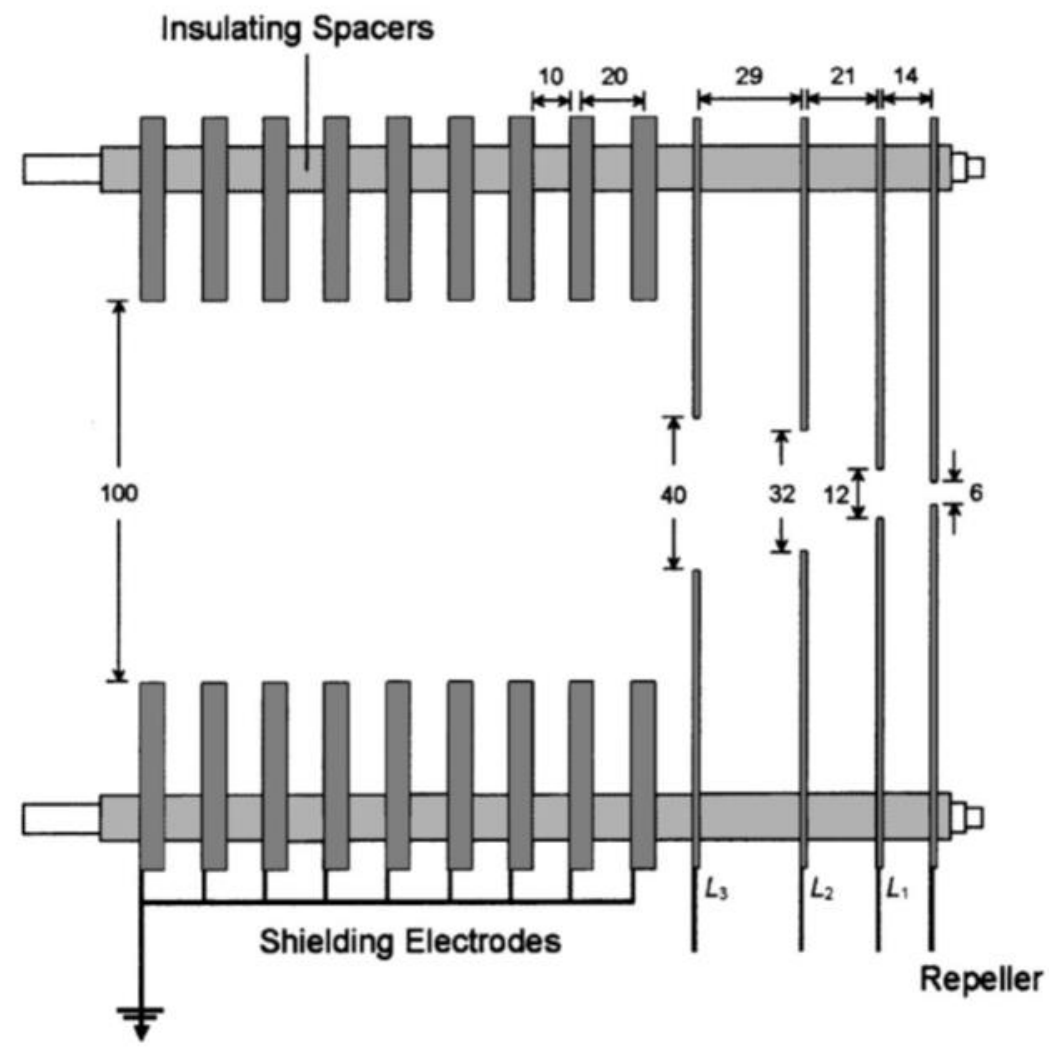

Fig. 2.3 Schematic of the ion lens assembly containing the four electrodes repeller, extractor $\left(\mathrm{L}_{1}\right)$, focusing lens $1\left(\mathrm{~L}_{2}\right)$, and focusing lens $2\left(\mathrm{~L}_{3}\right)$. Units are in $\mathrm{mm}$. Adapted with permission from D. Townsend et al, Rev. Sci. Instrum., 74(4), 2530-2539 (2003) @ AIP.

After the final focusing lens of the ion optics, a series of grounding electrodes were included to ensure there is no electric field penetrating into the TOF tube. Mu-metal also lines the TOF tube to protect against interfering magnetic fields. At the end of the TOF tube is a detector 
composed of a $75 \mathrm{~mm}$ MCP coupled to a P-47 phosphor screen (BOS-75-OPT01, Beam Imaging Solutions). The front plate of the MCP was grounded, while the back plate was pulsed to $2.1 \mathrm{kV}$ from a bias of $1.2 \mathrm{kV}$, and the phosphor voltage was $4.3 \mathrm{kV}$. Images were captured using a USB CCD camera (iDS uEye UI-2230SE-M-GL) and in-house data acquisition software NuACQ. The experiment was performed at $10 \mathrm{~Hz}$ with laser, valve, detector, and camera timings maintained by a Berkeley Nucleonics Delay Generator (model 577). Image reconstruction and data extraction were done using our finite slice analysis method, FinA. ${ }^{111,112}$ A PMT was also used to monitor and record TOF signal on a Keysight InfiniiVision DSOX3024A, 200 MHz, 4 GSa/s oscilloscope. These, in conjunction with a LabView program written in house wre used to record PHOFEX and REMPI spectra. The pump laser consisted of frequency doubled light from a dye laser (Sirah Cobra-Stretch, model CBST-LG-24) pumped by an Nd:YAG (Continuum Precision II, model PR9020). The probe laser consisted of frequency tripled light from a dye laser (Sirah CobraStretch, model CBST-LG-24) pumped by an Nd:YAG (Spectra-Physics Quanta-Ray, model LAB190-10H). The geometry of the experiment, Fig. 2.4, is that of a typical pump-probe photofragment imaging approach. The experimental configuration was exactly the same as in Fig. 2.4, except a dye laser and frequency doubling stage were added in front of the Nd:YAG to achieve the necessary pump photon energy.

Velocity distributions of imaged photoproduct ions are obtained using a $\mathrm{ms}^{-1} / \mathrm{pixel}$ calibration factor to convert camera pixels to velocity. A molecule with well-known ground and excited states, dissociation energy $\left(\mathrm{D}_{0}\right)$, and well-defined quantum states of photofragments is used to obtain the calibration factor. Here, the experimental setup was calibrated using OCS $+h v \rightarrow$ $\mathrm{CO}(\mathrm{v}, \mathrm{j})+\mathrm{S}\left({ }^{1} \mathrm{D}\right)$, with detection at $\mathrm{v}=0, j_{\mathrm{CO}}=47$ via $(2+1)$ REMPI by a $\mathrm{Q}(\mathrm{B} \leftarrow \mathrm{X})$ excitation. ${ }^{106}$ Internal energy of OCS following excitation and $\mathrm{D}_{0}$ are well-defined ${ }^{113,114}$ and the calibration 
experiment is "one-color", with the same laser used as dissociation and probe. If laser and ion focusing conditions are optimized, the velocity components of different sulfur isotopes can be resolved in $\mathrm{CO}$ images.

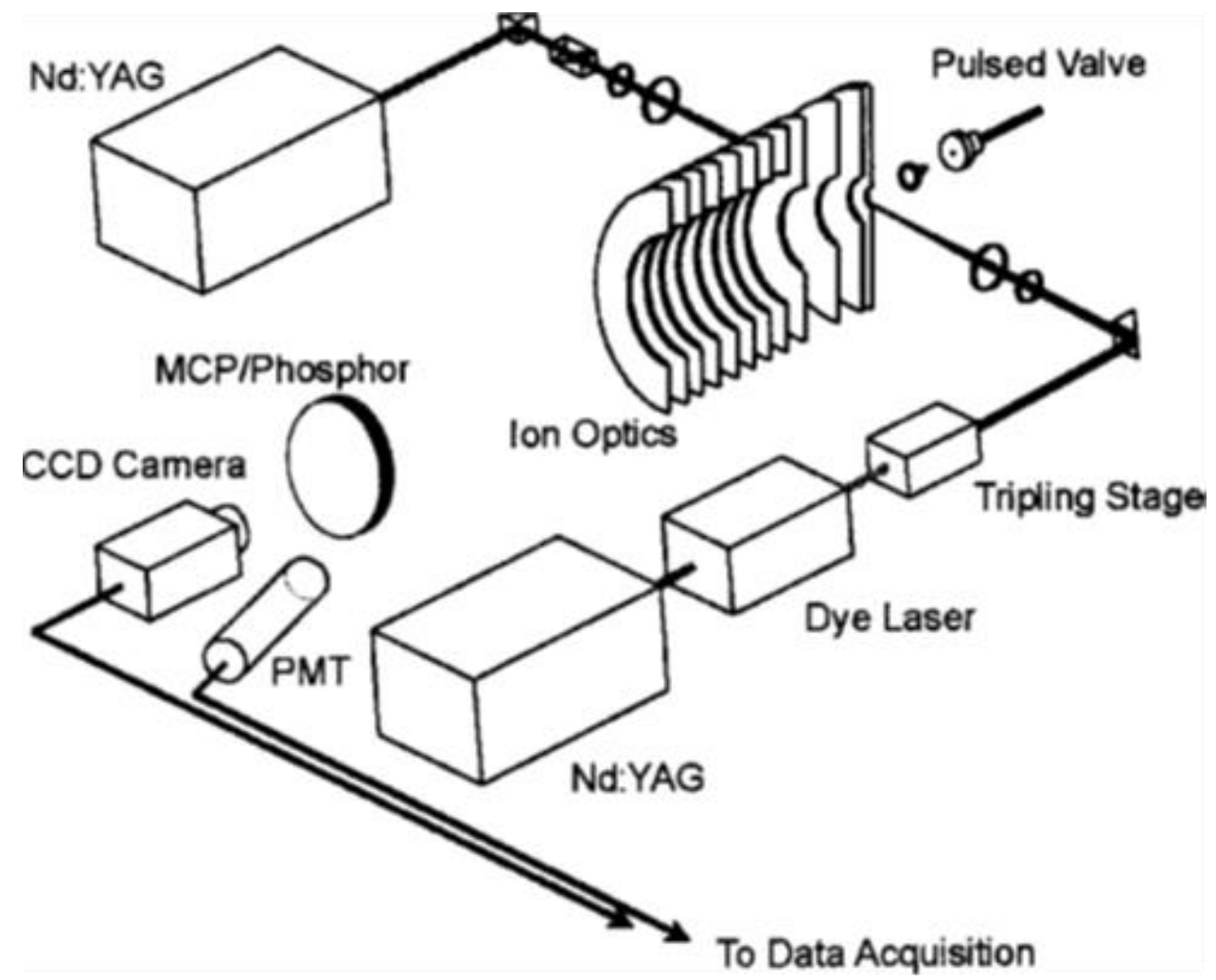

Fig. 2.4 Schematic of the experimental setup with a cut-through of the ion optics assembly. Adapted with permission from D. Townsend et al, Rev. Sci. Instrum., 74(4), 2530-2539 (2003) @ AIP. 


\section{CHAPTER 3}

\section{MIXED TRANSITIONS IN THE UV PHOTODISSOCIATION OF PROPARGYL CHLORIDE}

\subsection{Introduction}

Photodissociation dynamics of alkyl halides continue to garner interest after decades of research. ${ }^{15-121}$ Extensive experimental evidence of the photodissociation of methyl halides has revealed that dissociation via the first allowed electronic transition occurs directly from the n $\sigma^{*}$ state. ${ }^{122-125}$ Though the primary dissociation process in methyl halides is $\mathrm{C}-\mathrm{X}$ bond fission, $\mathrm{HX}$ elimination has been suggested as a channel for $\mathrm{CH}_{3} \mathrm{I}^{126}$ and was later reported in the photodissociation of $\mathrm{CH}_{3} \mathrm{Cl}$ following excitation via the second absorption band. ${ }^{127}$ Larger alkyl halides have more complex dissociation dynamics ${ }^{116,128-130}$ and for $\pi$-bonded systems, there is an alternative set of possibilities due to the presence of multiple chromophores and excited states. $^{131,132}$ Decreasing the degree of saturation, as in vinyl chloride, nearly removes the contribution from n $\sigma^{*}$ and $\pi \sigma^{*}$ to the predominantly $\pi \pi^{*}$ excited state prior to $\mathrm{C}-\mathrm{Cl}$ bond breaking from the excited state. ${ }^{133}$ Allyl chloride also has multiple dissociation pathways with at least two $\mathrm{HCl}$ elimination channels and two mechanisms of liberating $\mathrm{Cl} .{ }^{134}$ All referral to $\mathrm{Cl}$ here indicates the $\mathrm{Cl}^{35}$ isotope. For triple-bonded molecules such as propargyl chloride with two $\pi$ systems, one coplanar with the carbon-halogen backbone, one perpendicular to it, interesting effects on the electronic structure and dissociation dynamics can be expected.

The first strong absorption in propargyl chloride has a maximum at $185 \mathrm{~nm}$, so this transition is readily accessed at $193 \mathrm{~nm}$ where it has been studied in some detail. Both $\mathrm{Cl}$ and $\mathrm{HCl}$ products have been detected. ${ }^{135-137}$ In 1984 Kawasaki and coworkers used PTS to study the photodissociation of a range of halogenated hydrocarbons, including propargyl chloride. ${ }^{135}$ In that 
system they found $\mathrm{m} / \mathrm{z}=35\left(\mathrm{Cl}^{+}\right)$sharply peaked at high translational energies, while $\mathrm{m} / \mathrm{z}=36$ showed both low and high translational energy peaks. The high translational energy peak was attributed to momentum-matched $\mathrm{C}_{3} \mathrm{H}_{3}{ }^{+}$while the low energy portion was attributed to the $\mathrm{HCl}$ product. Overall, the fast fragments were attributed to fragmentation directly from a repulsive n $\sigma^{*}$ state, while the slow $\mathrm{HCl}$ was considered the result of intersystem crossing to a triplet state from the initially prepared $\pi \pi^{*}$ state. No product branching was reported because it was not possible to isolate the $\mathrm{HCl}^{+}$from the $\mathrm{C}_{3}{ }^{+}$arising from $\mathrm{C}_{3} \mathrm{H}_{3}$ cracking upon electron impact ionization.

In 1996, Butler and coworkers used emission spectroscopy and configuration interactionsingles ab initio calculations to investigate the contributions of various electronic configurations to the nominal $\pi \pi^{*}$ excited state in unsaturated hydrocarbon chlorides. ${ }^{133}$ Vinyl chloride and cisallyl chloride showed emission features analogous to ethylene, dominated by $\mathrm{C}=\mathrm{C}$ stretch suggesting the clear $\pi \pi^{*}$ nature of the excitation. In propargyl chloride, both the $\mathrm{C}-\mathrm{Cl}$ stretch and $\mathrm{C}=\mathrm{C}$ stretch were prominent in the emission spectra, indicating that the molecule must initially be in an excited state with a high degree of $\sigma^{*}$ character. Ab initio calculations clearly supported these results, with vinyl chloride and cis-allyl chloride showing fairly simple $\pi^{*}$ excited state character, while for propargyl chloride the transition was best described as excitation from the in-plane $\pi$ orbital to a mixture of $\pi^{*}$ and $\sigma^{*}$, with the latter dominant.

Soon after, Lee and Lin performed a PTS study at $193 \mathrm{~nm}$ in which they observed a slow component in the $\mathrm{m} / \mathrm{z}=35$ product, which they assigned exclusively to fragmentation of $\mathrm{HCl}$ on ionization, leading to inferred $\mathrm{Cl}: \mathrm{HCl}$ branching of 1:0.19. ${ }^{136}$ Harper et al. later found that the $\mathrm{Cl}+\mathrm{HCl}+$ fraction produced from electron impact ionization is 0.3 , considerably lower than the 0.72 value estimated by Lee and Lin. ${ }^{138}$ Furthermore, in 2003 Neumark and coworkers detected slow $\mathrm{C}_{3} \mathrm{H}_{3}$ fragments from propargyl chloride in photofragment translational spectroscopy 
experiments ${ }^{139}$ using tunable vacuum ultraviolet (VUV) photoionization. Butler later observed the same, ${ }^{137}$ likely due to improved S/N. Butler, Pratt, and coworkers sought to resolve the $\mathrm{HCl}$ elimination and $\mathrm{Cl}$ product channel branching using velocity map imaging and REMPI. ${ }^{137}$ Using state-specific detection, they were also able to determine the relative contributions of ground state $\mathrm{Cl}\left({ }^{2} \mathrm{P}_{3 / 2}\right)$ and spin-orbit excited $\mathrm{Cl}^{*}\left({ }^{2} \mathrm{P}_{1 / 2}\right)$ product channels. Approximately $95 \%$ of the $\mathrm{Cl}$ products were at high translational energy, in equal fractions of $\mathrm{Cl}$ and $\mathrm{Cl}^{*}$. Fragments at relatively low translational energy were only seen in the ground spin-orbit state, similar to what was previously observed in $\mathrm{Cl}$ photofragments of allyl chloride. ${ }^{115}$

Butler and coworkers also employed imaging with VUV $(9.67 \mathrm{eV})$ photoionization of the $\mathrm{C}_{3} \mathrm{H}_{3}$ product, and found a good match between the $\mathrm{Cl}$ and $\mathrm{C}_{3} \mathrm{H}_{3}$ data with a slow component (5\% in each).${ }^{137} \mathrm{~A}$ clear assignment of the slow $\mathrm{Cl}$ channel was not possible, but two explanations were considered: either dissociation from a lower-lying electronic state of propargyl chloride, or formation of electronically excited propargyl radicals. $\mathrm{HCl}$ elimination was also detected using electron impact ionization and estimated to be between $10 \%$ and $30 \%$ branching for propargyl chloride dissociation at $193 \mathrm{~nm}$. None of the several $\mathrm{C}_{3} \mathrm{H}_{2}$ isomers first produced as a cofragment of $\mathrm{HCl}$ were identified.

In a related study around the same time, Fan and Pratt examined propargyl bromide dissociation at $193 \mathrm{~nm}$ with imaging detection of $\mathrm{Br}$ and $\mathrm{Br}^{*}$ and tunable VUV ionization of $\mathrm{C}_{3} \mathrm{H}_{3} .{ }^{140}$ All distributions were bimodal, with a strong peak around $10 \mathrm{kcal}^{*} \mathrm{~mol}^{-1}$ and a weak peak around $35 \mathrm{kcal}^{*} \mathrm{~mol}^{-1}$. The $\mathrm{C}_{3} \mathrm{H}_{3}$ data could be matched well assuming a $0.3 \mathrm{Br}+0.7 \mathrm{Br} *$ branching. Tunable VUV from 8.97 to $9.86 \mathrm{eV}$ was used to detect $\mathrm{C}_{3} \mathrm{H}_{3}$ both from $\mathrm{C}_{3} \mathrm{H}_{3} \mathrm{Br}$ and $\mathrm{C}_{3} \mathrm{H}_{3} \mathrm{Cl}$, but no clear variation was seen in the spectra. A later investigation of $\mathrm{C}-\mathrm{Br}$ bond fission dynamics with dissociation at $234 \mathrm{~nm}$ observed high and low kinetic energy components corresponding to 
two dissociation channels. ${ }^{141}$ The high kinetic energy channel was explained as direct dissociation from a singlet $\sigma^{*}$ state while the low kinetic energy channel was attributed to internal conversion to a lower lying triplet state. A highly mixed excited state character based on $\mathrm{Br}$ angular distributions was also reported.

Propargyl chloride photodissociation studies have so far primarily sought to determine the branching in dissociation channels that originate from the strong absorption near $193 \mathrm{~nm}$. Here, we present an imaging investigation of propargyl chloride dissociation at longer wavelengths, with state-specific detection using REMPI probe of $\mathrm{Cl}$ and $\mathrm{Cl}^{*}$ and $1+1$ ionization of $\mathrm{C}_{3} \mathrm{H}_{3}$. The experimental data is interpreted with the aid of multireference calculations to characterize the nature of the electronic excitations.

\subsection{Experimental Methods}

A molecular beam containing $7 \% \mathrm{C}_{3} \mathrm{H}_{3} \mathrm{Cl}$ was generated by passing helium through $\mathrm{C}_{3} \mathrm{H}_{3} \mathrm{Cl}$ in a bubbler at $0{ }^{\circ} \mathrm{C}$. UV radiation at wavelengths near 212 and $236 \mathrm{~nm}$ was focused onto the molecular beam with a $30 \mathrm{~cm}$ focal length fused silica lens. These wavelengths were chosen for specific $\mathrm{Cl}$ and $\mathrm{Cl}^{*} \mathrm{REMPI}$ transitions: $\mathrm{For} \mathrm{Cl}$ and $\mathrm{Cl}^{*}$ the experiments are all one-color. For $\mathrm{Cl}$ detection the transitions were ${ }^{2} \mathrm{D}_{3 / 2}^{\circ} \leftarrow{ }^{2} \mathrm{P}_{3 / 2}^{\circ}\left(84988.48 \mathrm{~cm}^{-1}\right)$ and ${ }^{2} \mathrm{P}_{3 / 2}^{\circ} \leftarrow^{2} \mathrm{P}_{3 / 2}^{\circ}\left(94314.21 \mathrm{~cm}^{-}\right.$

${ }^{1}$ ), while for $\mathrm{Cl}^{*}$, the transitions were ${ }^{2} \mathrm{P}_{3 / 2}^{\circ} \leftarrow^{2} \mathrm{P}^{\circ}{ }_{1 / 2}\left(84560.08 \mathrm{~cm}^{-1}\right)$ and ${ }^{2} \mathrm{P}_{3 / 2}^{\circ} \leftarrow^{2} \mathrm{P}^{\circ}{ }_{1 / 2}(93431.85$ $\left.\mathrm{cm}^{-1}\right) \cdot{ }^{142,143}$ In each case, the wavelength was scanned repeatedly across the Doppler profile while recording the images. For $\mathrm{C}_{3} \mathrm{H}_{3}$ detection, the light was detuned slightly from the $\mathrm{Cl}$ or $\mathrm{Cl}^{*}$ transition. All UV light was polarized vertically, parallel to the plane of the detector. Image reconstruction and data extraction, including anisotropy parameters such as $\beta$, were done using FinA. ${ }^{111,112}$ This analysis has been shown to yield accurate angular distributions, giving $\beta$ values 
within 0.02 typically. Uncertainties in the energy-dependent $\beta$ values are dominated by statistics as may be inferred from the noise in the plots.

Theoretical calculations were performed in which the global minima on both singlet and triplet ground state potential energy surfaces of $\mathrm{C}_{3} \mathrm{H}_{3} \mathrm{Cl}$ were optimized at the $\operatorname{CCSD}(\mathrm{T})$ level with the cc-pVTZ basis for $\mathrm{H}$ and $\mathrm{C}^{144}$ and augcc-pVTZ for $\mathrm{Cl}^{145,146}$ (designated as aug(Cl)-cc-pVTZ), followed by frequency calculations in the harmonic approximation. The singlet-triplet separation was thus determined including correction for zero-point energy (ZPE). Single-point calculations at the $\operatorname{CCSD}(\mathrm{T})$ optimized geometry of the singlet ground state ${ }^{1} \mathrm{~A}^{\prime}$, which belongs to the $\mathrm{C}_{\mathrm{s}}$ group, were performed with various multireference methods to assess the energy levels in the FranckCondon (FC) region. Complete active space self-consistent field (CASSCF) ${ }^{147,148}$ calculations were used with the wave function state-averaged over 12 states $\left(3 \times{ }^{1} A^{\prime}, 3 \times{ }^{1} A^{\prime \prime}, 3 \times{ }^{3} A^{\prime}, 3 \mathrm{x}\right.$ ${ }^{3} A^{\prime \prime}$, "SA12-CASSCF"). Several active spaces were tested, i.e. 14 electrons in 13 orbitals ( $8 a^{\prime}$ and $\left.5 a^{\prime \prime}\right), 14$ electrons in 11 orbitals ( $7 a^{\prime}$ and $\left.4 a^{\prime \prime}\right), 10$ electrons in 11 orbitals ( $7 a^{\prime}$ and $\left.4 a^{\prime \prime}\right)$, and 10 electrons in 9 orbitals ( $6 a^{\prime}$ and $\left.3 a^{\prime \prime}\right)$. For both $14 / 11$ and 10/9 active spaces, the CASSCF wave functions were used to perform XMS-CASPT2 calculations (multi-state complete active space, second order perturbation theory) in order to treat dynamical electronic correlation (imaginary level shift of 0.4 a.u.), ${ }^{149}$ while the CASSCF(10/9) wave function was also used for MRCI-F12 calculations, which provides here the most accurate evaluation of the energy levels after Davidson's correction. ${ }^{150}$ Additionally, two types of CASSCF scans were performed. First, $\operatorname{CASSCF}(10 / 9)$ single point calculations along the $\mathrm{q}_{14}$ vibrational mode, an antisymmetric CCC bend located at $301 \mathrm{~cm}^{-1}$ at the $\operatorname{CSSD}(\mathrm{T})$ level (and likely to be significantly populated), were carried out in order to evaluate which states might exhibit vibronic couplings due to the occurrence of double-wells along this coordinate, as pointed out by Eisfeld in the case of propargyl radical. ${ }^{151}$ 
The wave function was state-averaged over the 6 first singlet states. Second, CASSCF(10/11) single point calculations were performed along the $\mathrm{C}-\mathrm{Cl}$ coordinate, with geometry relaxed on the singlet ground state at the MP2 level of theory. ${ }^{152}$ In this case the wave function was state averaged over the six singlets and six triplets states mentioned above, which merge in two mixed asymptotes as $\mathrm{R} \rightarrow \infty$, i.e. $\mathrm{C}_{3} \mathrm{H}_{3}+\mathrm{Cl}$ and $\mathrm{C}_{3} \mathrm{H}_{3} *+\mathrm{Cl}$. For both CASSCF scans, the cc-pVTZ basis set was used for $\mathrm{H}, \mathrm{C}$, and $\mathrm{Cl}$. All calculations were performed with MOLPRO. ${ }^{153}$ The calculations are summarized in Table 3.1, and structures of ${ }^{1} A^{\prime \prime}$ and ${ }^{3} A$ minima are shown in Fig. 3.1. Energy, ZPE, Cartesian coordinates and vibrational frequencies of the ${ }^{1} A^{\prime \prime},{ }^{3} A$, and ${ }^{3} A^{\prime \prime}$ minima can be found in Appendix A.

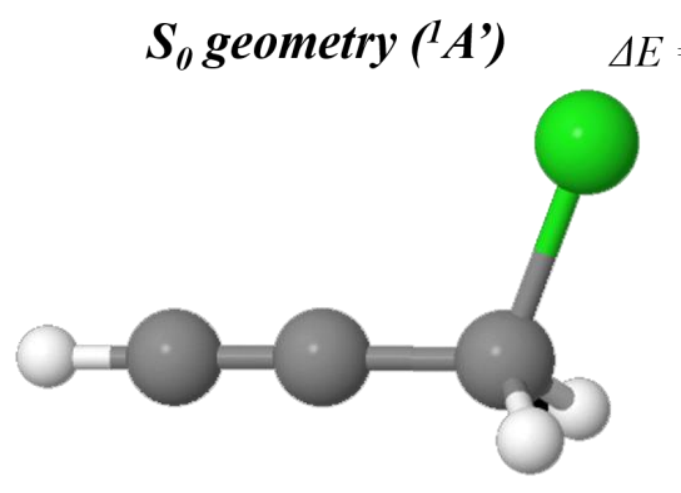

side view

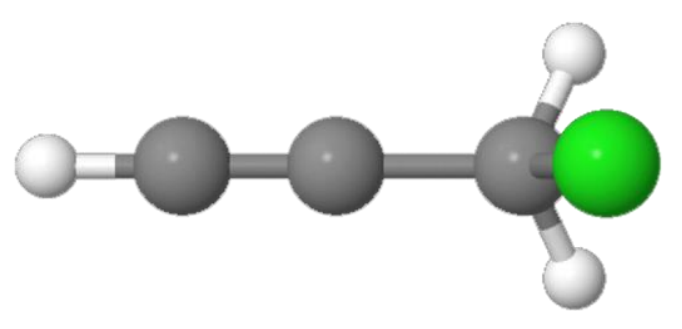

top view

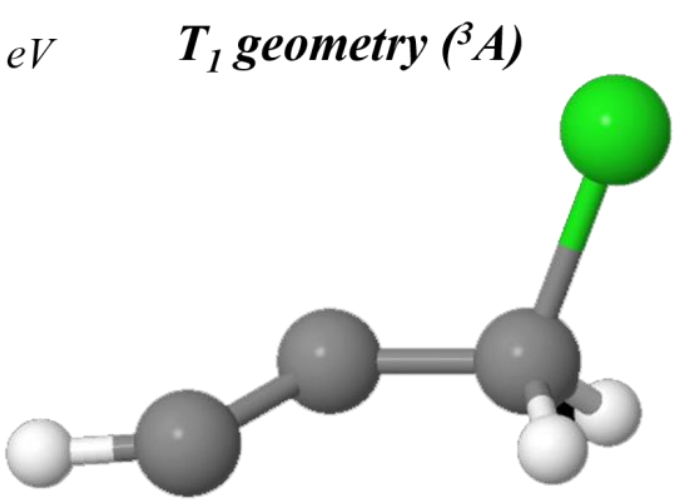

side view

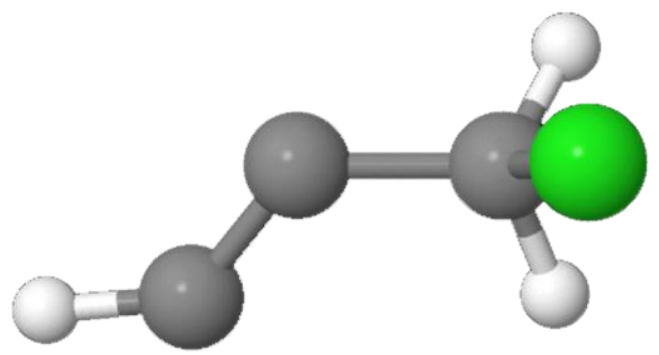

top view

Fig. 3.1 Structures of the ${ }^{1} \mathrm{~A}^{\prime}$ and ${ }^{3} \mathrm{~A}$ minima optimized at the $\operatorname{CCSD}(\mathrm{T})$ level, along with singlet-triplet separation energy, $\triangle \mathrm{E}$, including $\mathrm{ZPE}$ correction. 


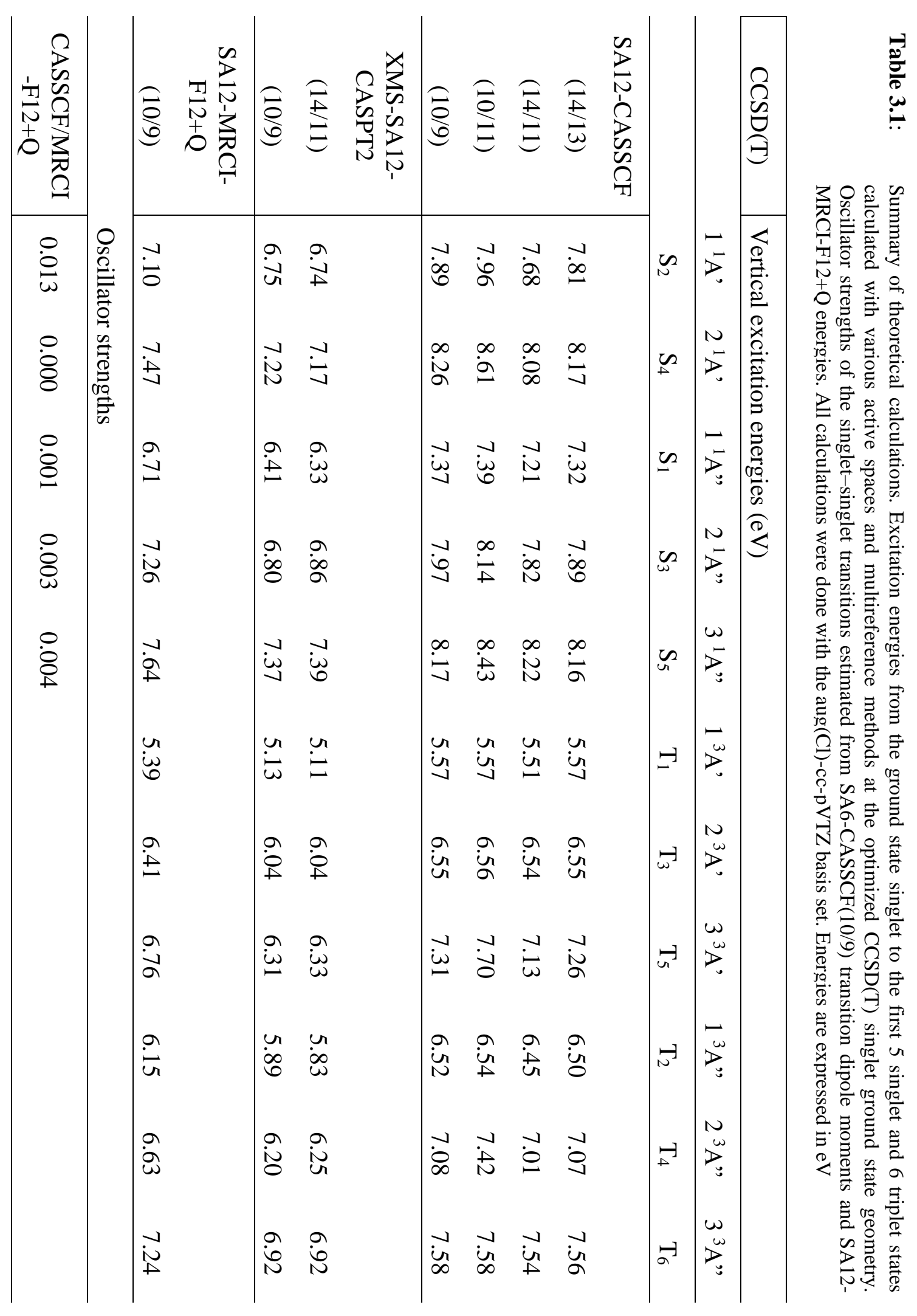




\subsection{Results and Discussion}

We first present imaging results for UV dissociation of propargyl chloride near $236 \mathrm{~nm}$. Fig. 3.2 shows DC slice images obtained for detection of ground state $\mathrm{Cl}$ atom at $235.3 \mathrm{~nm}$ and for spin-orbit excited $\mathrm{Cl}^{*}$ at $236.5 \mathrm{~nm}$. The $\mathrm{Cl}$ image in Fig. 3.2A exhibits two components: a broad inner ring and a sharp outer ring. Both regions show recoil preferentially oriented along the laser polarization direction. The $\mathrm{Cl}^{*}$ image in Fig. 3.2B shows a single ring, which is wider on the sides than on the poles. This unusual feature arises from an inner perpendicular distribution overlapping with an outer nearly isotropic distribution. Fig. 3.3A and B give the total translational energy distributions $\mathrm{P}\left(\mathrm{E}_{\mathrm{T}}\right)$ obtained from the images in Fig. 3.2A and B, respectively. Superimposed on
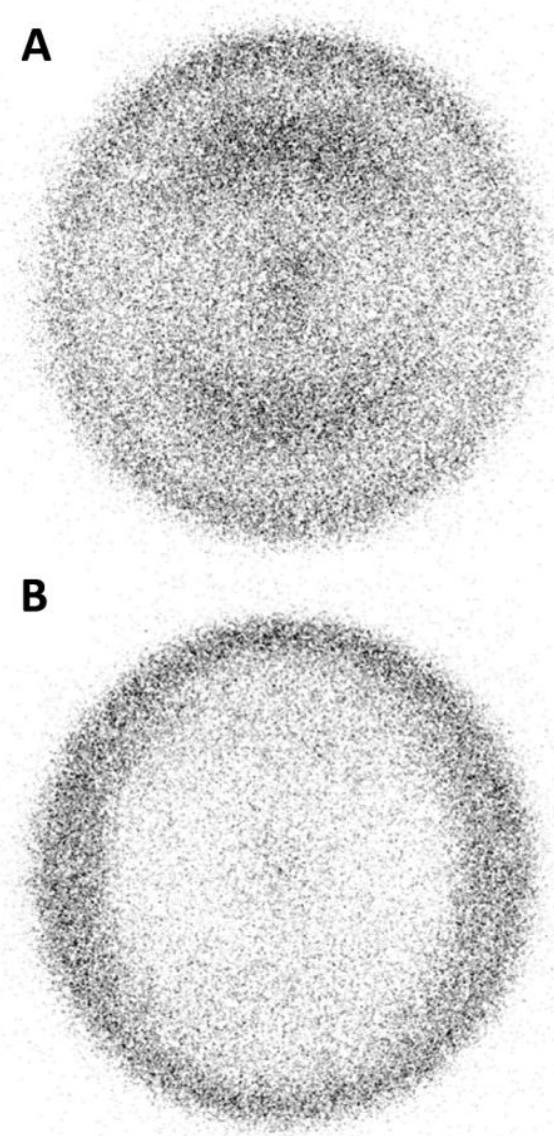

Fig. 3.2 DC slice images of $\mathrm{Cl}(\mathrm{A})$ and $\mathrm{Cl} *(\mathrm{~B})$ from photodissociation of propargyl chloride near $236 \mathrm{~nm}$. Laser polarization axis is vertical. 
the TEDs in Fig. 3.3A and B are the associated angular distributions given as a function of translational energy.

We first examine the $\mathrm{Cl}^{*}$ distributions, as they allow to understand the $\mathrm{Cl}$ distributions more clearly. The inner, perpendicular component of the $\mathrm{Cl}^{*}$ TEDs appears as a shoulder with a maximum around $20 \mathrm{kcal} \mathrm{mol}^{-1}$, with the primary peak reaching a maximum around $26 \mathrm{kcal} \mathrm{mol}^{-}$ 1. The corresponding $\beta$ values reach a minimum of -0.5 near the peak of the inner shoulder, but then rise to slightly positive values. The ground state $\mathrm{Cl}$ TEDs appears bimodal as we have noted above. The angular distributions of $\mathrm{Cl}$ and $\mathrm{Cl}^{*}$ show clear similarities for the outer peak. Ignoring
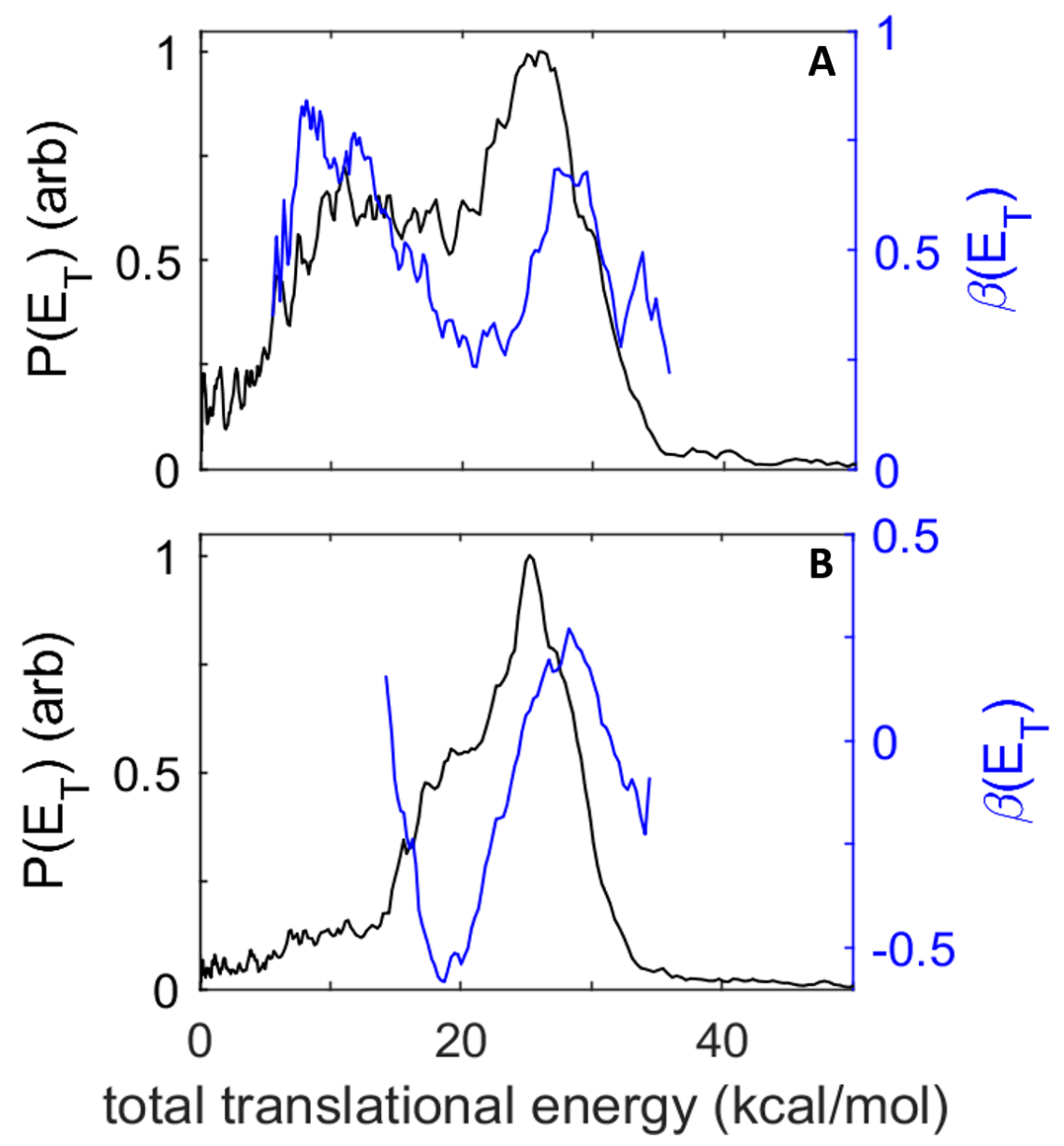

Fig. 3.3 Translational energy distributions (black) and anisotropy parameter $\beta$ (blue) of $\mathrm{Cl}$ (A) and $\mathrm{Cl}^{*}$ (B) from photodissociation of propargyl chloride near $236 \mathrm{~nm}$. 
for the moment the distinct low energy peak around $10 \mathrm{kcal} \mathrm{mol}^{-1}$, the main peak shows the same trend as $\mathrm{Cl}^{*}$, with a minimum in $\beta$ around $20 \mathrm{kcal} \mathrm{mol}^{-1}$ that rises sharply to a substantially more parallel value, although for the $\mathrm{Cl}$ case all $\beta$ values are positive. This suggests that the main peak is also composite, consisting of overlapping components peaking around 20 and $26 \mathrm{kcal} \mathrm{kcal} \mathrm{mol}^{-}$ ${ }^{1}$ just as for $\mathrm{Cl}^{*}$. The additional lower energy peak is even more strongly parallel, with $\beta$ values approaching +0.8 .

Fig. 3.4 shows DC slice images for photodissociation near 212 nm. Fig. 3.4A is the sliced image for $\mathrm{Cl}$, Fig. 3.4B is $\mathrm{Cl} *$ and Fig. $3.4 \mathrm{C}$ is an image obtained at $\mathrm{m} / \mathrm{z}=39, \mathrm{C}_{3} \mathrm{H}_{3}$. We did not detect $\mathrm{m} / \mathrm{z}=39$ in the vicinity of the $236 \mathrm{~nm}$ excitation. The ground state $\mathrm{Cl}$ image shows a fast, sharp ring that appears somewhat perpendicular, along with a weaker slow isotropic feature barely visible inside the fast, sharp ring. The $\mathrm{Cl}^{*}$ Image shows a bimodal distribution with features that appear to be largely isotropic. The $\mathrm{C}_{3} \mathrm{H}_{3}$ image shows two main components that are somewhat parallel: A fast outer ring that matches with the $\mathrm{Cl}$ and $\mathrm{Cl}^{*}$ images and a sharp inner ring that does not appear in the $\mathrm{Cl}$ atom images. The secondary inner ring that appears in the $\mathrm{Cl}^{*}$ image does not appear clearly in the $\mathrm{C}_{3} \mathrm{H}_{3}$ images. The translational energy and energy-dependent angular distributions derived from the images in Fig. 3.4 are shown in Fig. 3.5. The $\mathrm{Cl}$ distribution shows the main fast peak around $32 \mathrm{kcal} \mathrm{mol}^{-1}$, tapering to lower energy suggesting some evidence of a second component as in the $\mathrm{Cl}^{*}$ image. That inner component shows a $\beta$ near zero, but the main peak is consistently perpendicular $(\beta=-0.3)$. The $\mathrm{Cl}^{*}$ distribution has an analogous fast feature, along with a distinct component associated with the inner ring. The angular distribution overall is largely isotropic but varies from $\beta=-0.2$ to 0.2 across the two components. The $\mathrm{C}_{3} \mathrm{H}_{3}$ TEDs can be seen to be a combination of a component momentum-matched to the $\mathrm{Cl}$ and $\mathrm{Cl}^{*}$ and a distinct 
feature peaking around $10 \mathrm{kcal} \mathrm{mol}^{-1}$ mentioned above. The energy dependent $\beta$ values range from near 0 to around 0.4 .
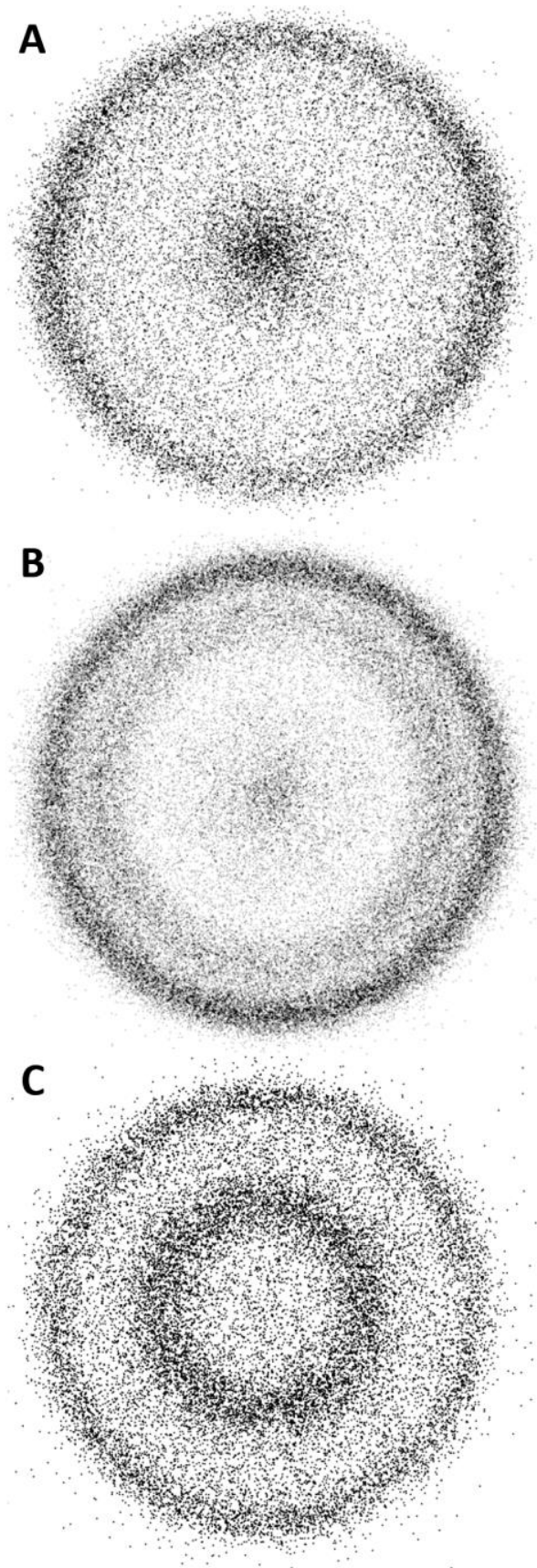

Fig. 3.4 DC slice images of $\mathrm{Cl}(\mathrm{A}) \mathrm{Cl}^{*}(\mathrm{~B})$ and $\mathrm{C}_{3} \mathrm{H}_{3}(\mathrm{C})$ from photodissociation of propargyl chloride near $212 \mathrm{~nm}$. Laser polarization axis is vertical. 

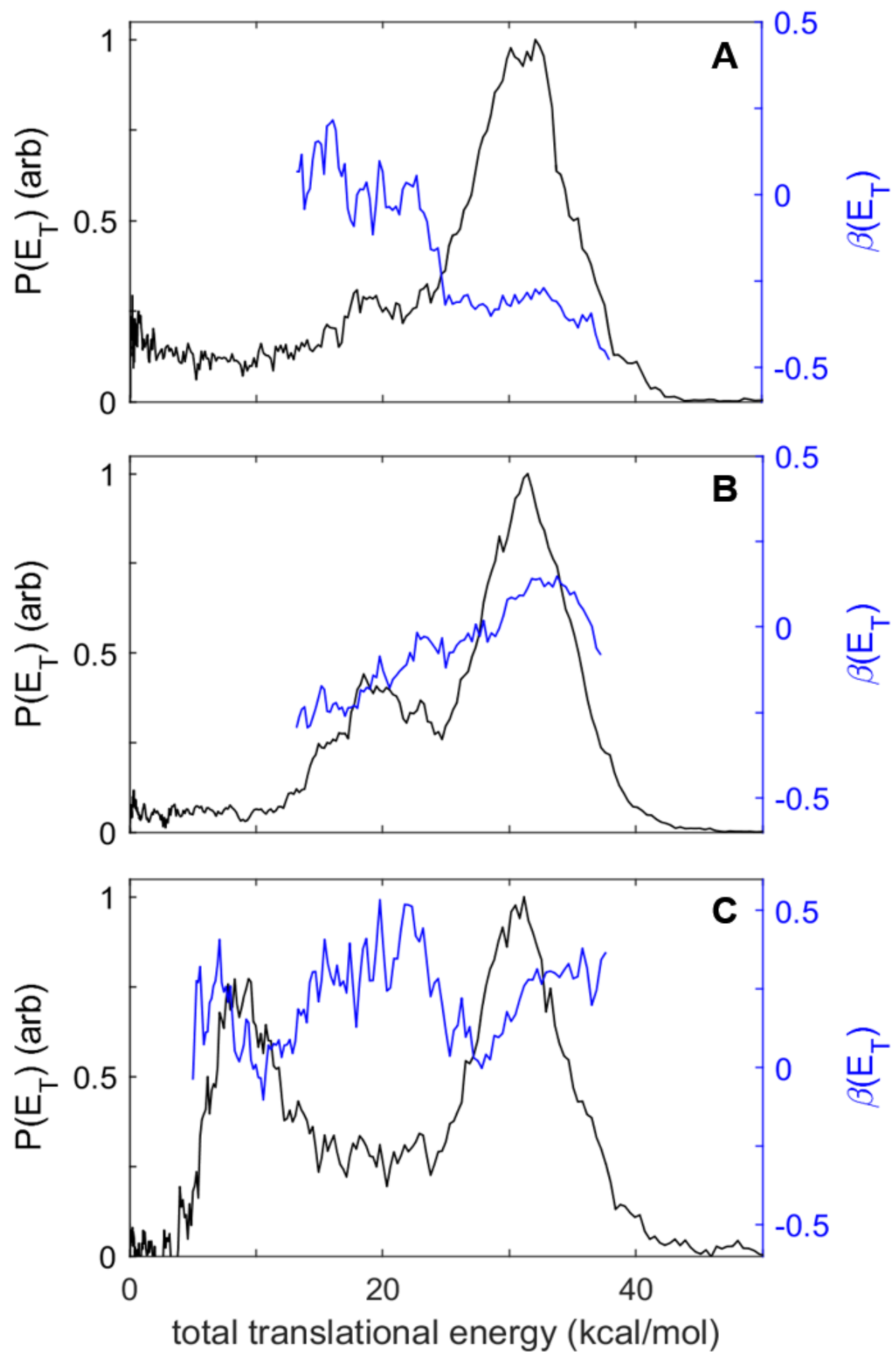

Fig. 3.5 Translational energy distributions (black) and anisotropy parameter $\beta$ (blue) of $\mathrm{Cl}$ (A) $\mathrm{Cl}^{*}$ (B) and $\mathrm{C}_{3} \mathrm{H}_{3}$ (C) from photodissociation of propargyl chloride near $212 \mathrm{~nm}$. 
Theoretical calculations of the propargyl radical absorption spectrum by Eisfeld indicate a strong $2{ }^{2} B_{1} \leftarrow 1^{2} B_{1}$ electronic absorption beginning around $335 \mathrm{~nm}$, and a much weaker one, identified as $3{ }^{2} B_{1} \leftarrow 1^{2} B_{1}$, extending from $240 \mathrm{~nm}$ to $185 \mathrm{~nm}$, with a vertical maximum near 206 nm. ${ }^{151}$ We believe the fast outer ring in the $\mathrm{C}_{3} \mathrm{H}_{3}$ image in Fig. $3 \mathrm{C}$ results from $1+1$ REMPI via this $3{ }^{2} B_{1} \leftarrow 1^{2} B_{1}$ transition. The relatively intense inner feature in the $\mathrm{C}_{3} \mathrm{H}_{3}$ that does not match the $\mathrm{Cl}$ data must have a different origin entirely. As discussed below, we can readily assign this feature to 2-photon dissociative ionization of propargyl chloride yielding the aromatic $\mathrm{c}-\mathrm{C}_{3} \mathrm{H}_{3}{ }^{+}$ ion with $\mathrm{Cl}$, while the sharp cut-off on the low-energy side likely reflects secondary decomposition of the hot $\mathrm{c}-\mathrm{C}_{3} \mathrm{H}_{3}{ }^{+}$ion.

The absorption spectrum recorded by Fahr et al. for propargyl chloride (Fig. 3.6) is reproduced to guide the following discussion. ${ }^{6,154}$ Marked on the figure are the two wavelength regions examined here in addition to the well-studied $193 \mathrm{~nm}$ excimer line. For propargyl chloride, the first strong absorption appears at $185 \mathrm{~nm}$, while for propargyl bromide this excitation is shifted to slightly longer wavelength. ${ }^{6,154}$ In addition, for propargyl bromide there is a weak broad maximum around $230 \mathrm{~nm}$ that is up to two orders of magnitude weaker in the propargyl chloride spectrum, strongly suggesting a triplet excitation present in this vicinity. The results of our multireference calculations at the MRCI-F12/aug(Cl)-cc-pVTZ level, also shown in Fig. 3.6, confirm the presence of a series of low-lying triplet states, the first of which, $1^{3} A^{\prime}\left(\mathrm{T}_{1}\right)$ has a vertical excitation energy of $5.39 \mathrm{eV}$, which is very near the photon energy at $236 \mathrm{~nm}$. Following $\mathrm{T}_{1}$ are a pair of states, $1^{3} A^{\prime \prime}$ and $2{ }^{3} A^{\prime}\left(\mathrm{T}_{2}\right.$ and $\left.\mathrm{T}_{3}\right)$ very close at 6.15 and $6.41 \mathrm{eV}$, respectively. There are additional triplets at higher energy, but for our purpose the next transition of importance is likely the first singlet excited state $1{ }^{1} A^{\prime \prime}\left(\mathrm{S}_{1}\right)$ at $6.71 \mathrm{eV}$. We note the vertical transitions to the lower singlet states appear at higher energy than the associated features in the spectrum. This is 
ascribed to vibronic coupling, likely involving the antisymmetric $\mathrm{CCC}$ bending mode ( $\left.\mathrm{v}_{14}\right)$ along which $S_{1}, S_{2}, S_{3}$, and $S_{4}$ are found to exhibit double-wells (see Fig. 3.7).

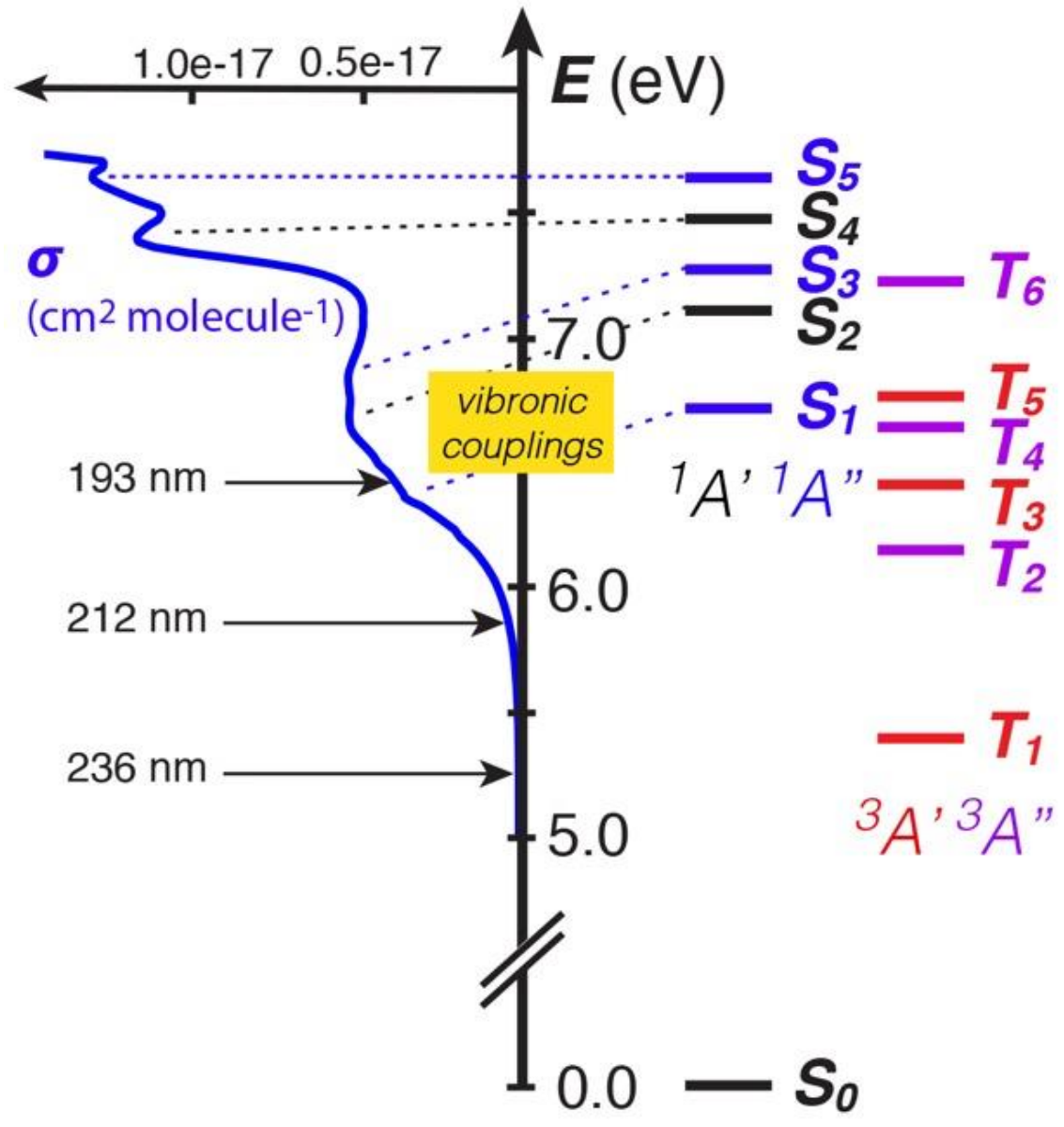

Fig. 3.6 Absorption spectrum of propargyl chloride (left, blue) at $295 \mathrm{~K}$ (adapted from Fahr et al.) and excitation energies of the singlet and triplet states located below $8 \mathrm{eV}\left(3 \times 1{ }^{\prime} A^{\prime}\right.$ (black), $3 \times 1{ }^{\prime \prime} A^{\prime \prime}$ (blue), $3 \times{ }^{3} A^{\prime}$ (red), $3 \times 3^{3} A^{\prime \prime}$ (purple), calculated at the MRCI-F12/aug(Cl)-cc-pVTZ level. Laser excitation wavelengths are shown with black arrows and a tentative assignment of the features observed in the experimental spectrum with dashed lines. Note that strong vibronic couplings are expected for excitations to the $S_{1}, S_{2}, S_{3}$, and $S_{4}$ states due in part to the presence of double-wells along the low-frequency CCC bending coordinate (see Fig. 3.7).

In the following, we refer to the potential energy cuts given in Fig. 3.8. We first consider the inner component of the ground state $\mathrm{Cl}$ product at $236 \mathrm{~nm}$, which peaks at $11 \mathrm{kcal} \mathrm{mol}^{-1}$ total 


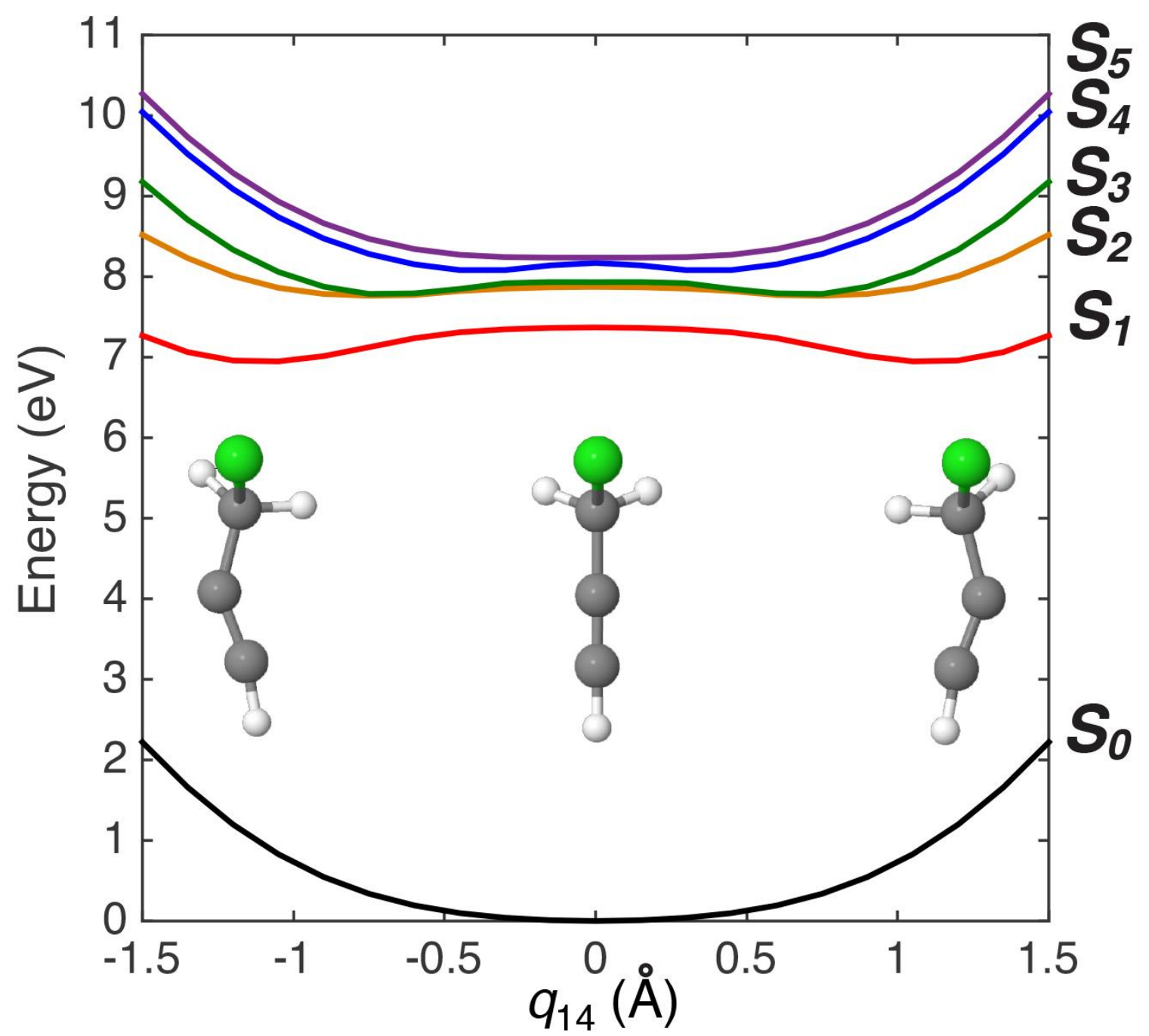

Fig. 3.7 Potential energies of the ground state $S_{0}$ and the five first singlet excited states calculated at the SA6-CASSCF(10/9)/cc-pVTZ level along the $\mathrm{q}_{14}$ normal mode.

translational energy and shows considerable anisotropy. There is no related contribution in the $\mathrm{Cl}^{*}$ distribution, and as we move to shorter wavelength, this component disappears entirely. We assign this contribution to excitation to the $T_{1}$ state, which results in ground state products and does not cross another potential surface along the reaction coordinate. We then find the two nearly overlapping peaks that appear both in $\mathrm{Cl}$ and $\mathrm{Cl}^{*}$ images and TEDs. In this case, for $\mathrm{Cl}^{*}$ the inner peak is strongly perpendicular, while the outer peak is predominantly parallel. In fact, the same trend is seen for the $\mathrm{Cl}$ data, but shifted to higher $\beta$ values. This suggests excitations that branch 
to both products, with the perpendicular component branching more to $\mathrm{Cl}^{*}$. We can thus clearly associate the perpendicular contribution with the $1{ }^{3} A^{\prime \prime}$ state, $\mathrm{T}_{2}$, and the outer, parallel component with the $2{ }^{3} A^{\prime}$ state, $T_{3}$. According to the calculations, these are separated by only $0.26 \mathrm{eV}$ in the FC region. A crossing of these surfaces is seen in Fig. 3.8, with the lower $1^{3} A^{\prime \prime}$ likely preferentially following a nonadiabatic path to the $\mathrm{Cl}^{*}$ asymptote.

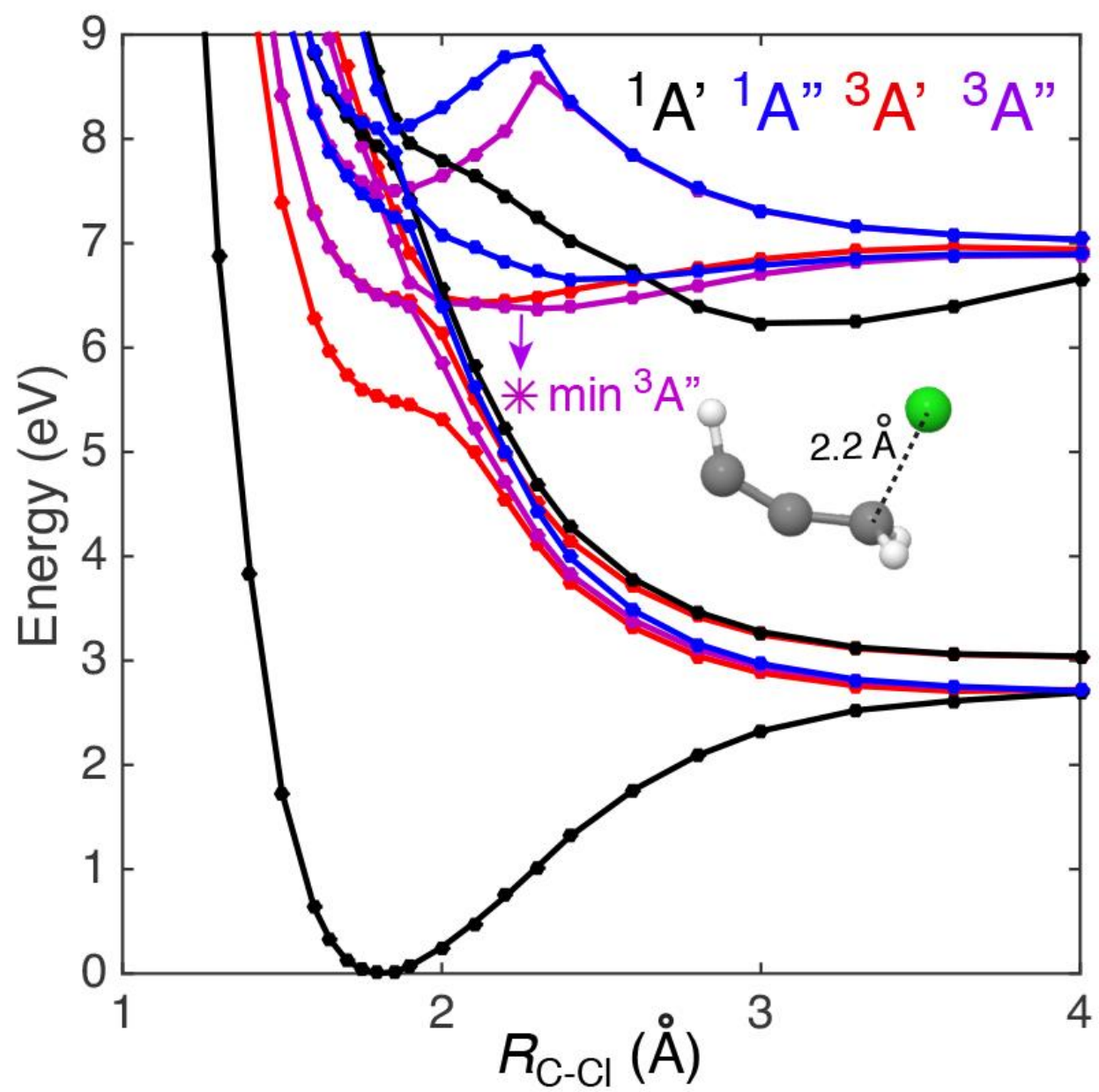

Fig. 3.8 Potential energy cuts along the $\mathrm{RC}-\mathrm{Cl}$ coordinate leading to the $\mathrm{C} 3 \mathrm{H} 3+\mathrm{Cl}$ and $\mathrm{C}_{3} \mathrm{H}_{3} *+\mathrm{Cl}$ asymptotes. The level of theory is SA12- CASSCF(10/11)/ccpVTZ and the geometry is relaxed on the ground state by optimization at the MP2/cc-pVTZ level. The color code for state symmetry and multiplicity is the same as in Fig. 5. The molecular structure corresponds to a minimum on the $2^{3} A^{\prime \prime}$ state, which correlates to the $\mathrm{C} 3 \mathrm{H} 3 *+\mathrm{Cl}$ asymptote and could play a role as an intermediate before the dissociation occurs towards the ground state. 
Around $212 \mathrm{~nm}$, the striking aspect of the data is that the angular distributions are now nearly isotropic except for the main peak for the $\mathrm{Cl}$ case. Moreover, for the $\mathrm{Cl}^{*}$, two well-separated components appear, peaking at 20 and $32 \mathrm{kcal} \mathrm{mol}^{-1}$. We assign these to predissociation, likely by the same triplet states, following excitation to the bound $\mathrm{S}_{1}$ state. Considering the $\mathrm{C}_{3} \mathrm{H}_{3}{ }^{+}$data, the outer region agrees well with the $\mathrm{Cl} / \mathrm{Cl}^{*}$ data, and we ascribe this signal to ionization of the propargyl radical photoproduct by $1+1$ ionization through $3{ }^{2} B_{2} \leftarrow 1^{2} B_{2}$ transition, which Eisfeld finds has a maximum around $220 \mathrm{~nm} .{ }^{151}$ It is not clear, however, whether there is important vibrational selectivity in this REMPI process. The good agreement between the $\mathrm{Cl} / \mathrm{Cl}^{*}$ and $\mathrm{C}_{3} \mathrm{H}_{3}{ }^{+}$ signals on the shape of the outer peak suggests there is no strong selectivity over the levels produced there. The bimodal distribution observed in the $\mathrm{Cl}^{*}$ data is much weaker in the $\mathrm{Cl}$ distribution and although not readily apparent in the image, it shows up in the integrated distribution. The anisotropy seen in the $\mathrm{Cl}$ image is not present in the $\mathrm{Cl}^{*}$ nor in the $\mathrm{C}_{3} \mathrm{H}_{3}{ }^{+}$, suggesting this is the result of angular momentum alignment that modulates the sensitivity in these one-color experiments. Indeed, in separate two-color experiments to be reported elsewhere we find evidence for alignment in the $\mathrm{Cl}$ atom following dissociation in the deep $\mathrm{UV}$, but not at $230 \mathrm{~nm}$ or longer. The puzzling aspect of reconciling the $\mathrm{Cl} / \mathrm{Cl} *$ data and the $\mathrm{C}_{3} \mathrm{H}_{3}$ result is that the latter angular distributions appear overall somewhat parallel and do not match any combination of the $\mathrm{Cl}$ and $\mathrm{Cl}^{*}$ distributions. Given our lack of a detailed understanding of the $\mathrm{C}_{3} \mathrm{H}_{3}$ ionization process and the noise in the data, we place less confidence in the $\mathrm{C}_{3} \mathrm{H}_{3}$ angular distributions.

The intense peak at $10 \mathrm{kcal} \mathrm{mol}^{-1}$ in the $\mathrm{C}_{3} \mathrm{H}_{3}{ }^{+}$has no corresponding contribution in the $\mathrm{Cl}$ signals, therefore it does not arise from ionization of a $\mathrm{C}_{3} \mathrm{H}_{3}$ photoproduct. Instead, it results from 2-photon dissociative ionization of propargyl chloride via the $S_{1}$ state. The total two-photon energy here is $11.58 \mathrm{eV}$. The appearance energy of $\mathrm{C}_{3} \mathrm{H}_{3}{ }^{+}$from propargyl chloride has been reported as 
$11.02 \mathrm{eV}$, and the product ion in this case has been identified as the cyclopropenium cation. ${ }^{155}$ Given the $0 \mathrm{~K}$ heats of formation of $\mathrm{c}_{-} \mathrm{C}_{3} \mathrm{H}_{3}{ }^{+}\left(256.9 \mathrm{kcal} \mathrm{mol}^{-1}\right), \mathrm{Cl}\left(28.6 \mathrm{kcal} \mathrm{mol}^{-1}\right)$ and $\mathrm{C}_{3} \mathrm{H}_{3} \mathrm{Cl}$ $\left(44.6 \mathrm{kcal} \mathrm{mol}^{-1}\right),{ }^{1,2,156}$ we find the available energy for this channel to be $1.18 \mathrm{eV}$ or $27 \mathrm{kcal} \mathrm{mol}^{-}$ ${ }^{1}$. The barrier for $\mathrm{Cl}$ elimination to form $\mathrm{c}-\mathrm{C}_{3} \mathrm{H}_{3}{ }^{+}$is indicated by the onset of the peak at $8 \mathrm{kcal} \mathrm{mol}^{-}$ ${ }^{1}$, in good agreement with the earlier estimate of $0.32 \mathrm{eV} .{ }^{155}$ An interesting aspect of the $2{ }^{3} A^{\prime \prime}, \mathrm{T}_{4}$ state is the potential well correlated with the $\mathrm{C}_{3} \mathrm{H}_{3} *+\mathrm{Cl}$ asymptote. The molecular structure of this minimum is displayed in Fig. 6 and we note this as a possible long-lived intermediate before dissociation occurs towards the ground state products.

These results may be contrasted with the $193 \mathrm{~nm}$ dissociation studied by Butler and others. ${ }^{137}$ In that case, both $\mathrm{Cl}$ and $\mathrm{Cl}^{*}$ appeared as a strong peak at $40 \mathrm{kcal} \mathrm{mol}^{-1}$ that was slightly parallel, while a much weaker contribution at $8 \mathrm{kcal} \mathrm{mol}^{-1}$ was seen in the $\mathrm{Cl}$ only. Based on the theoretical calculations presented here, we can ascribe the primary peak to the allowed $S_{2}$ absorption consistent with the earlier assignments. The minor peak seen at $8 \mathrm{kcal} \mathrm{mol}^{-1}$ that appears in $\mathrm{Cl}$ (and propargyl) likely arises from a distinct transition and gives rise to propargyl radical in the ${ }^{2} B_{2}$ first excited state. An analogous feature is seen much more strongly in propargyl bromide dissociation suggesting perhaps that it arises from one of the triplet excitations in this region.

The repulsive dissociation dynamics observed suggest a simple model of internal energy partitioning, and we follow here the treatment of Fan and Pratt for propargyl bromide. ${ }^{140}$ For an initially rotation-less parent molecule, the final rotational excitation of the propargyl radical must be equal in magnitude to the exit orbital angular momentum. If we assume impulsive energy release along the $\mathrm{C}-\mathrm{Cl}$ bond, and direct recoil between the two fragments from this geometry, then in a classical picture we can relate the final rotational energy to the recoil energy as 


$$
E_{\text {rot }}=\frac{\mu_{R-C l} b^{2}}{I} E_{T}
$$

where $\mathrm{m}$ is the reduced mass of the propargyl-Cl system, $\mathrm{b}$ the exit impact parameter, and I the moment of inertia of the propargyl radical about the $\mathrm{C}$ axis. Using $140 \mathrm{pm}$ for $b$ based on the

calculated propargyl chloride geometry, and the propargyl radical moment of inertia $8.83 \times 10^{-46}$ $\mathrm{kg}^{*} \mathrm{~m}^{2}$, we find $\mathrm{E}_{\mathrm{rot}}=0.68 \mathrm{E}_{\mathrm{T}}$. This allows us to estimate the rotational excitation accompanying each peak, and we can attribute the balance of the available energy to vibration. The results for each identified $\mathrm{Cl}$ and $\mathrm{Cl}^{*}$ peak are given in Table 3.2. The fraction of available energy appearing in vibration ranges from $13 \%$ for the fast peak to $64 \%$ for the slow peak for $\mathrm{Cl}$ at $236 \mathrm{~nm}$, corresponding to 7 to $34 \mathrm{kcal} \mathrm{mol}^{-1}$, respectively. Similar values are obtained for $\mathrm{Cl}^{*}$. We can obtain one estimate of the possible vibrational excitation in the propargyl radical simply by calculating the relaxation energy from the geometry of the parent molecule, which we find to be $11.7 \mathrm{kcal} \mathrm{mol}^{-1}$. Values in Table 3.2 that are much higher than this suggest impulsive vibrational excitation for the direct dissociation processes, with energy randomization also possibly contributing for the predissociation near $212 \mathrm{~nm}$.

\subsection{Conclusion}

DC slice imaging experiments have uncovered complex dissociation dynamics of propargyl chloride at 212 and $236 \mathrm{~nm}$ involving both triplet and singlet excitations. High-level theoretical calculations were used to characterize the electronic states necessary to interpret the TEDs of $\mathrm{Cl}$ and $\mathrm{Cl}^{*}$ images and their associated anisotropy. Photoexcitation to the $\mathrm{T}_{1}$ state at $236 \mathrm{~nm}$ results in dissociation to ground state $\mathrm{Cl}$ and $\mathrm{C}_{3} \mathrm{H}_{3}$ exclusively. Photoexcitation at $236 \mathrm{~nm}$ also accesses $\mathrm{T}_{2}$ and $\mathrm{T}_{3}$, whose overlapping excitations branch to both $\mathrm{Cl}$ and $\mathrm{Cl}^{*}$. The perpendicular component of the excitation is associated with the $1^{3} A^{\prime \prime}$ state, $\mathrm{T}_{2}$, and preferably branches to $\mathrm{Cl}^{*}$, while the 
parallel component is associated with the $2^{3} A^{\prime}$ state, $T_{3}$. Photoexcitation at $212 \mathrm{~nm}$ initially accesses the bound $\mathrm{S}_{1}$ state followed by predissociation by triplet states. The repulsive dissociation dynamics likely cause impulsive vibrational excitation from direct dissociation and energy randomization in predissociation processes. Multireference ab initio calculations are essential to decipher the complex dynamics involving this high density of singlet and triplet excited electronic states. 


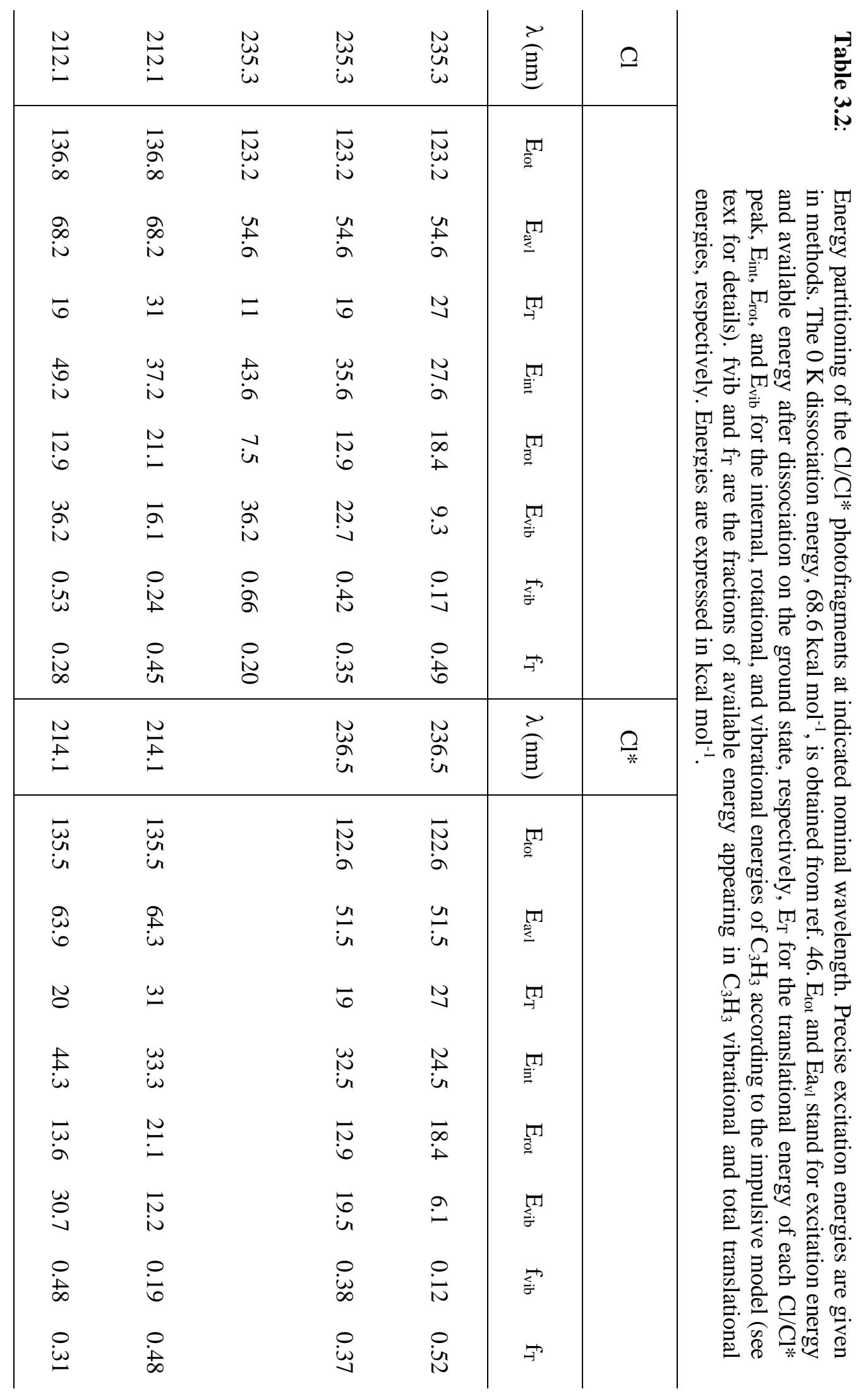




\section{CHAPTER 4}

\section{INFRARED MULTIPHOTON EXCIATION AND DISSOCIATION OF PROPARGYL CHLORIDE}

\subsection{Introduction}

High-power line-tunable transverse excitation atmospheric (TEA)- $\mathrm{CO}_{2}$ lasers stimulated the emergence of IRMPD studies in the 1970s and 1980s. ${ }^{157-166}$ This permitted the investigation of ground state unimolecular decomposition of molecules under collisionless conditions in molecular beams. ${ }^{37,55,167-170}$ Although applied with considerable success to reveal the global features of these processes, the methods were in wide use prior to the development of state-selected velocity map imaging techniques. ${ }^{99,101,103}$ The latter method is capable of extremely high velocity resolution and at the same time quantum state-specific product detection, affording deep insight into photochemical processes in favorable cases. ${ }^{171-175}$

Our interest in combining IRMPD with imaging is inspired in part by a desire to explore roaming radical reactions in detail. Although $\mathrm{NO}_{3}$ dissociation clearly showed the importance of roaming in the excited electronic state, ${ }^{37,38}$ in general roaming reactions are more easily seen and characterized on the ground electronic state. IRMPD combined with velocity map imaging thus offers a means to explore this novel aspect of reaction dynamics in detail. Recently, we began adapting IRMPD for high resolution DC slice imaging applications. ${ }^{37,176-178}$ In IRMPD of vinyl chloride, for example, state-resolved $\mathrm{HCl}$ detection showed both modest rotational excitation and very low translational energy release, consistent with 3-center $\mathrm{HCl}$ elimination producing cold vinylidene as a coproduct. ${ }^{178}$ Propargyl chloride experiments have primarily sought to determine the branching of dissociation channels that originate from the strong excitation near $193 \mathrm{~nm} .^{135-137}$ Butler and coworkers summarized photodissociation of propargyl chloride and other halogenated 
hydrocarbons in their imaging study in $2006 .{ }^{137} \mathrm{C}-\mathrm{Cl}$ bond fission products were found to branch to $\mathrm{Cl}$ and $\mathrm{Cl}^{*}$ equally and slow translational energy peaks were determined not to be the result of $\mathrm{HCl}$ fragmentation as previously thought. The previous chapter presented an imaging investigation at longer UV wavelengths that used multireference calculations to characterize the electronic excitations of propargyl chloride from 5.0 to $8.0 \mathrm{eV} .{ }^{40} \mathrm{~A}$ high density of singlet and triplet excited electronic states were identified and complex, repulsive dissociation dynamics were observed with both direct and predissociation processes. At $236 \mathrm{~nm}$ three distinct triplet excited states were accessed giving distinct features in the images. A slow component producing exclusively ground state products with a parallel angular distribution was assigned to $T_{1}$. Evidence for $T_{2}$ and $T_{3}$ excitations were also seen in a fast peak comprising distinct perpendicular and parallel components, respectively. Furthermore, $\mathrm{T}_{2}$ and $\mathrm{T}_{3}$ preferentially branched to spin-orbit excited and ground state $\mathrm{Cl}$, respectively. Evidence was also seen for predissociation of a bound $\mathrm{S}_{1}$ state accessed when propargyl chloride was excited at $212 \mathrm{~nm}$. Here, we combine IRMPE of propargyl chloride with state-specific detection using REMPI with DC slice imaging to examine the dynamics of dissociation both on the ground state near threshold and in vibrationally mediated photodissociation through these electronically excited states. The experimental data is interpreted using theoretical calculations to determine minima and transition states en route to products and aid in identifying the $\mathrm{C}_{3} \mathrm{H}_{2}$ product isomer formed. We also performed complementary chirped-

pulse mm-wave spectroscopy experiments in a quasi-uniform flow (CPUF) which support the computational identification of the $\mathrm{C}_{3} \mathrm{H}_{2}$ product isomer as the linear propadienylidene species.

\subsection{Experimental Methods}

A molecular beam containing $7 \% \mathrm{C}_{3} \mathrm{H}_{3} \mathrm{Cl}$ was generated by passing helium through $\mathrm{C}_{3} \mathrm{H}_{3} \mathrm{Cl}$ in a bubbler at $0{ }^{\circ} \mathrm{C}$. Infrared (IR) radiation from a grating-tuned $\mathrm{TEA}-\mathrm{CO}_{2}$ laser at 10.44 
$\mu \mathrm{m}$ was used to excite $\mathrm{C}_{3} \mathrm{H}_{3} \mathrm{Cl}$ by strong absorption via the $\mathrm{C}-\mathrm{C}$ stretch. ${ }^{7,179}$ A $35 \mathrm{~cm}$ focal length $\mathrm{ZnSe}$ lens was used to focus the measured $\sim 650 \mathrm{~mJ}$ per pulse (fluence $7.2 \mathrm{~J} \mathrm{~cm}^{2}$ ) onto the molecular beam slightly upstream of the UV laser. The IR beam was fired $\sim 800$ ns before the UV beam. UV radiation around $241 \mathrm{~nm}$ and $0.8 \mathrm{~mJ}$ per pulse was focused onto the molecular beam slightly downstream from the IR laser beam with a UV fused silica lens and used to ionize the $\mathrm{HCl}$ product by $(2+1)$ REMPI via the $\mathrm{R}$ and $\mathrm{S}$ branches of the $\mathrm{F}^{1} \Delta_{2} \leftarrow \leftarrow \mathrm{X}^{1} \Sigma^{+}$transition. ${ }^{107}$ Unlike other transitions in this region, this REMPI transition exclusively produces $\mathrm{HCl}^{+}$and minimal fragmentation. For the $\mathrm{Cl}$ detection experiments, UV radiation near $235 \mathrm{~nm}$ was focused onto the molecular beam with a $30 \mathrm{~cm}$ focal length fused silica lens. These wavelengths were chosen for specific $\mathrm{Cl}\left({ }^{2} \mathrm{P}_{3 / 2}\right)$ and $\mathrm{Cl} *\left({ }^{2} \mathrm{P}_{1 / 2}\right)$ REMPI transitions. For $\mathrm{Cl}$ detection the transition was ${ }^{2} \mathrm{D}^{\circ}{ }_{3 / 2} \leftarrow^{2} \mathrm{P}^{\circ}{ }_{3 / 2}\left(84988.48 \mathrm{~cm}^{-1}\right)$, while for $\mathrm{Cl}^{*}$ the transition was ${ }^{2} \mathrm{P}_{3 / 2}^{\circ} \leftarrow^{2} \mathrm{P}^{\circ}{ }_{1 / 2}\left(85442.43 \mathrm{~cm}^{-}\right.$ $\left.{ }^{1}\right) .{ }^{142,143}$ In each case, the wavelength was scanned repeatedly across the Doppler profile while recording the images. All UV light was polarized vertically, parallel to the plane of the detector. Image reconstruction and data extraction were performed using FinA ${ }^{111,112}$ in the same manner as the UV photodissociation of propargyl chloride.

CPUF spectroscopy experiments were used to confirm imaging results. The CPUF apparatus has been described before, but a brief description of relevant information is included here. ${ }^{180-182}$ The chirped-pulse mm-wave spectrometer covering the range $70-90 \mathrm{GHz}$ is coupled to a pulsed quasi-uniform flow. The propargyl chloride is seeded at $1 \%$ in helium and expanded from a Laval nozzle forming a quasi-uniform flow at a total density of $\sim 10^{16} \mathrm{~cm}^{-3}$ and a temperature of 10 to $40 \mathrm{~K}$. Photodissociation of the propargyl chloride by a loosely focused $193 \mathrm{~nm}$ laser beam ( $\sim 10 \mathrm{~mJ}$ per pulse) gives several products including the propargyl radical with $\mathrm{Cl}$ atoms, and $\mathrm{HCl}$ with $\mathrm{C}_{3} \mathrm{H}_{2}$. Absorption of a second $193 \mathrm{~nm}$ photon by the propargyl radical also gives $\mathrm{C}_{3} \mathrm{H}_{2}$ 
products, and the isomer-specific branching is the subject of studies reported elsewhere. ${ }^{183}$ The photoproducts are internally cooled in the flow, after which they are excited by $\pi / 2$ pulses of mmwave radiation tuned to known transitions of $\mathrm{C}_{3} \mathrm{H}_{2}$ isomers at time intervals of $10 \mu \mathrm{s}$. After each mm-wave pulse, the free induction decay of the excited molecules is acquired and accumulated in the time domain, then Fourier-transformed to give the rotational spectrum from which branching is determined as described previously. Results for propargyl chloride shown here are compared to results obtained previously for 1,2-butadiene to isolate the component that is associated with $\mathrm{HCl}$ elimination.

To explore the ground state potential energy surface of the $\mathrm{C}_{3} \mathrm{H}_{3} \mathrm{Cl}$ system, we employed the DFT functional wB97XD, ${ }^{184}$ which incorporates an empirical correction for long-range interactions, in conjunction with the aug-cc-pVDZ basis set. ${ }^{144-146}$ Further calculations were performed using the composite CBS-QB3 method. ${ }^{185-187}$ The structures of the minima and transition states were fully optimized and their vibrational frequencies estimated within the harmonic approximation. ${ }^{185,188}$ The transition states found in each pathway were confirmed by intrinsic reaction coordinate calculations. All calculations were performed with the Gaussian 16W package. ${ }^{188}$ Associated files are provided in Appendix B.

\subsection{Results and Discussion}

Ground state $\mathrm{Cl}$ atom images from photodissociation of propargyl chloride near $235 \mathrm{~nm}$ are first presented. Fig. 4.1A shows a DC slice image obtained with the UV probe laser alone (" 1 color') and Fig. 4.1B shows probe following the high-power $\mathrm{CO}_{2}$ laser pulse ('“2-color'). The 1color image is bimodal with a broad inner ring and a sharper outer ring with recoil along the laser polarization direction. As described in the previous chapter, we determined that the inner and outer 
rings were the result of excitation to triplet states of propargyl chloride, namely $\mathrm{T}_{1}$ and $\mathrm{T}_{2} / \mathrm{T}_{3}$, respectively. We discuss the mixed composition of the outer ring further below.
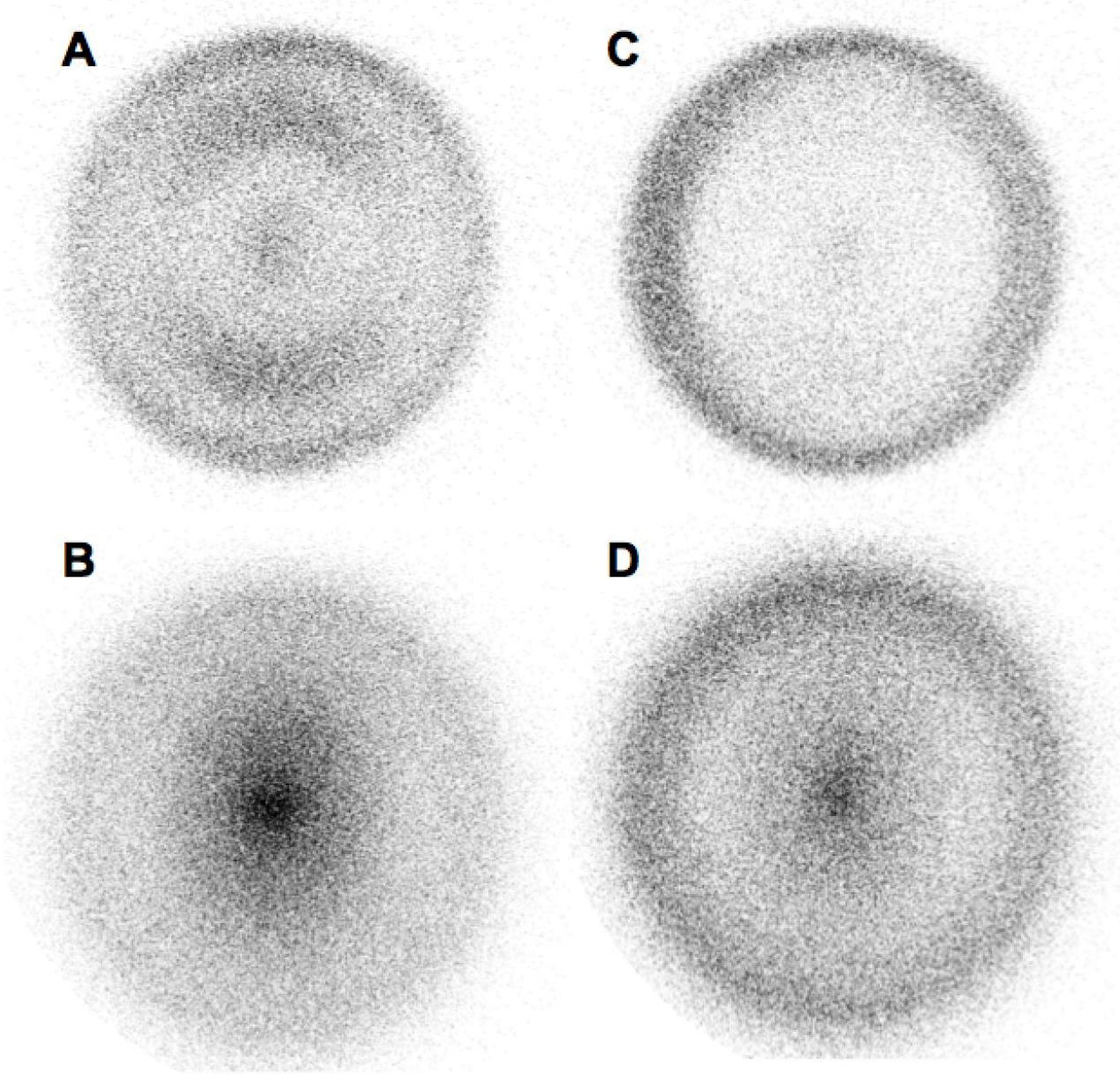

Fig. 4.1 DC slice images of 1-color $\mathrm{UV} \mathrm{Cl}(\mathrm{A}), 2$-color $\mathrm{Cl}(\mathrm{B}), 1$-color $\mathrm{UV} \mathrm{Cl}^{*}(\mathrm{C})$, and 2-color $\mathrm{Cl}^{*}$ (D) from photodissociation of propargyl chloride. Laser polarization axis is vertical in the plane of the figure.

The 2-color image shows profound changes. The total signal increases by roughly a factor of two and a varying enhancement is seen in the yield at all recoil energies, with the central region becoming very intense. It is clear this enhancement must be due, in large part, to IR-enhanced UV dissociation, since the enhancement closely follows the distributions we observed in the UV-only images. The effects may be seen more clearly in the TEDs displayed in Fig. 4.2A. The region we assigned previously to the $T_{1}$ excitation near $10 \mathrm{kcal} \mathrm{mol}^{-1}$ is enhanced by $\sim 30 \%$, while the main 
$T_{2} / T_{3}$ peak is not strongly enhanced. Instead, the distribution extends to a significantly higher limiting translational energy: rather than the sharp cutoff around $35 \mathrm{kcal} \mathrm{mol}^{-1}$, the distribution tails off slowly to greater than $50 \mathrm{kcal} \mathrm{mol}^{-1}$. Considering this entire distribution there is a similar large IR enhancement for the $T_{2} / T_{3}$ contribution but it is spread over a broad, higher energy range.
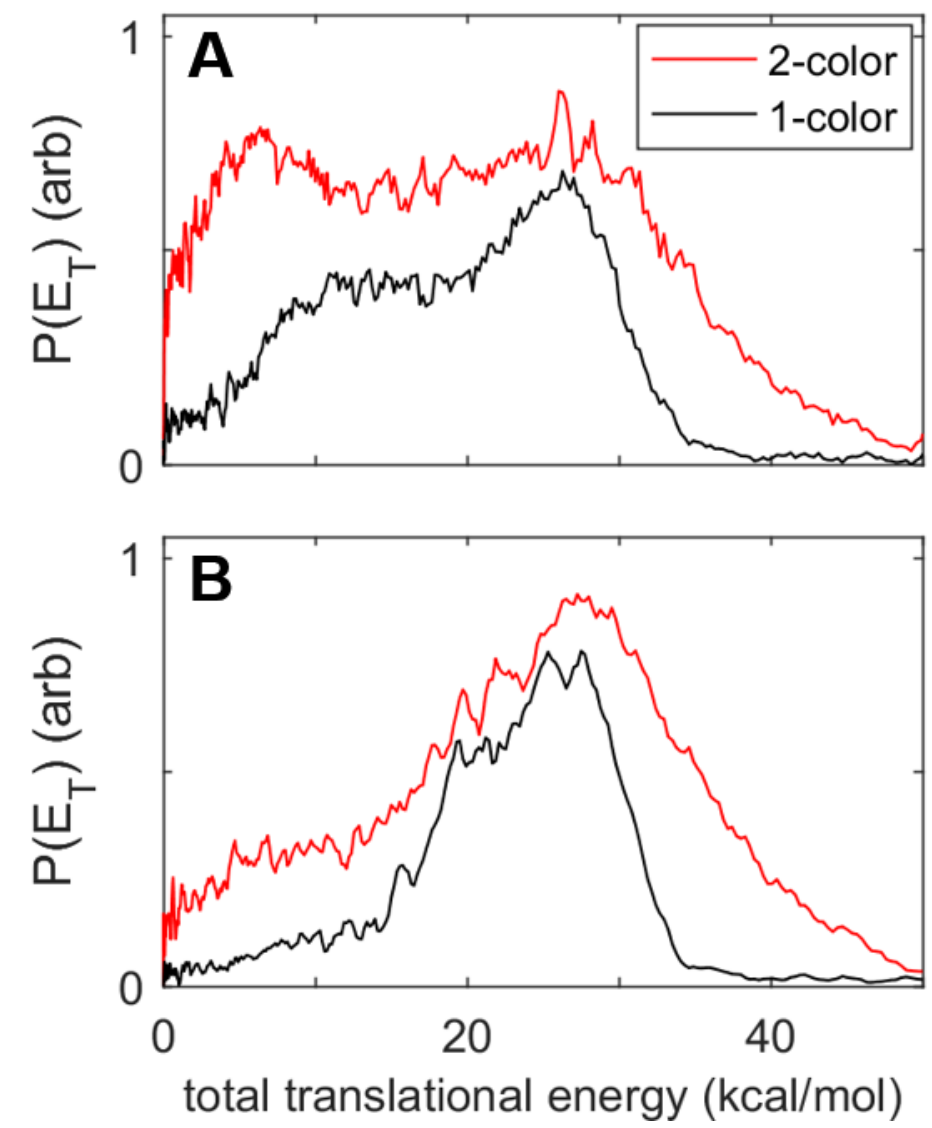

Fig. 4.2 Translational energy distributions of $\mathrm{Cl}(\mathrm{A})$ and $\mathrm{Cl}^{*}$ (B) from 1-color UV (black) and 2-color (red) photodissociation of propargyl chloride photodissociation.

We previously examined energy partitioning in the UV-only dissociation and found for the main peak that $\sim 45 \%$ of the available energy appeared in translation. If this fraction is maintained for this IR enhanced dissociation, then we can infer that the maximum excitation of the molecules that undergo IR enhanced dissociation via the $\mathrm{T}_{2} / \mathrm{T}_{3}$ excitation is $\sim 42 \mathrm{kcal} \mathrm{mol}^{-1}$, i.e., on the order of 16 IR photons. Interestingly, we do not see such a shift in the maximum translational energy for the $\mathrm{T}_{1}$ contribution. This is likely because the $\mathrm{C}-\mathrm{Cl}$ coordinate on $\mathrm{T}_{1}$ surface is not as steeply 
sloped in the $\mathrm{FC}$ region compared to $\mathrm{T}_{2} / \mathrm{T}_{3}$, so additional vibrational energy does not yield a greater impulse. Additional vibrational excitation does, however, increase the FC envelope to give greater excitation probability. The other very notable feature of the 2-color vs. 1-color images and TEDs for $\mathrm{Cl}$ is the prominent low energy peak that only appears in the 2-color result. This is a peak near $5 \mathrm{kcal} \mathrm{mol}^{-1}$ that we ascribe to IRMPD leading to $\mathrm{Cl}$ atom elimination with the UV serving only as probe.

Analogous results for $\mathrm{Cl}^{*}$ are shown in the Fig. 4.1C and D images, showing marked differences from the ground state $\mathrm{Cl}$ case. The 1-color $\mathrm{Cl}^{*}$ image does not show the $10 \mathrm{kcal} \mathrm{mol}^{-1}$ contribution assigned to $T_{1}$ excitation. It also shows an interesting structure in the main peak that is perpendicular for the inner component and parallel for the outer component, as discussed previously. We assigned the inner component to the $T_{2}$ excitation and the outer component to $T_{3}$ and argued that the former branches more to $\mathrm{Cl}^{*}$, accounting for the more strongly perpendicular aspect of the inner component of the main peak for $\mathrm{Cl}^{*}$ compared to $\mathrm{Cl}$. The 2-color results for $\mathrm{Cl}^{*}$ also show new and enhanced dissociation processes. There is a slight enhancement of the peaks corresponding to $T_{2}$ and $T_{3}$, and again a strong broad feature extending to $50 \mathrm{kcal} \mathrm{mol}^{-1}$ or so. The $\mathrm{Cl}^{*}$ data also exhibits a low energy feature around $5 \mathrm{kcal} \mathrm{mol}^{-1}$ consistent with IRMPD.

The $\mathrm{Cl}$ and $\mathrm{Cl}^{*}$ images indicate dissociation by IRMPD and concurrent IR-enhanced UV photodissociation. Analysis of the angular distributions at different regions of the image further assists in disentangling the contribution from each. $\beta$ is given as a function of recoil energy in Fig. 4.3 and 4.4. We reproduce the TEDs in these plots to facilitate the comparison. Fig. 4.3A gives the 1-color result for $\mathrm{Cl}$, Fig. 4.3B gives the 2-color result. The low translational energy release and nearly isotropic angular distribution of the image center supports the assignment of the slow component to IRMPD. The IR-enhanced photodissociation distribution which we assign to $\mathrm{T}_{1}$ 
shows a reduction in the anisotropy from $\beta \sim 0.7$ to 0.4 . We attribute this to rovibrational excitation of the parent induced by the $\mathrm{CO}_{2}$ laser leading to dissociation from the rotating parent molecule and from a wider range of geometries. For the region of the main peak around $20 \mathrm{kcal} \mathrm{mol}^{-1}$ that we associate primarily with $T_{2}$ excitation, there is little impact on the anisotropy although there is a significant enhancement in the signal. One possible explanation is that the excitation shifts more to $T_{3}$ which is more parallel, but at the same time there is a reduction in overall anisotropy, and these effects cancel. In the region of the main peak associated with $\mathrm{T}_{3}$, we again see a significant reduction in anisotropy, from $\beta \sim 0.7$ to 0.3 . However, beyond $35 \mathrm{kcal} \mathrm{mol}^{-1}$, the $\beta$ value rises significantly, to about 0.8 . We believe this indicates a contribution from $S_{2}$ now making an appearance, as direct excitation from distorted geometries becomes possible. $S_{2}$ is the first strongly
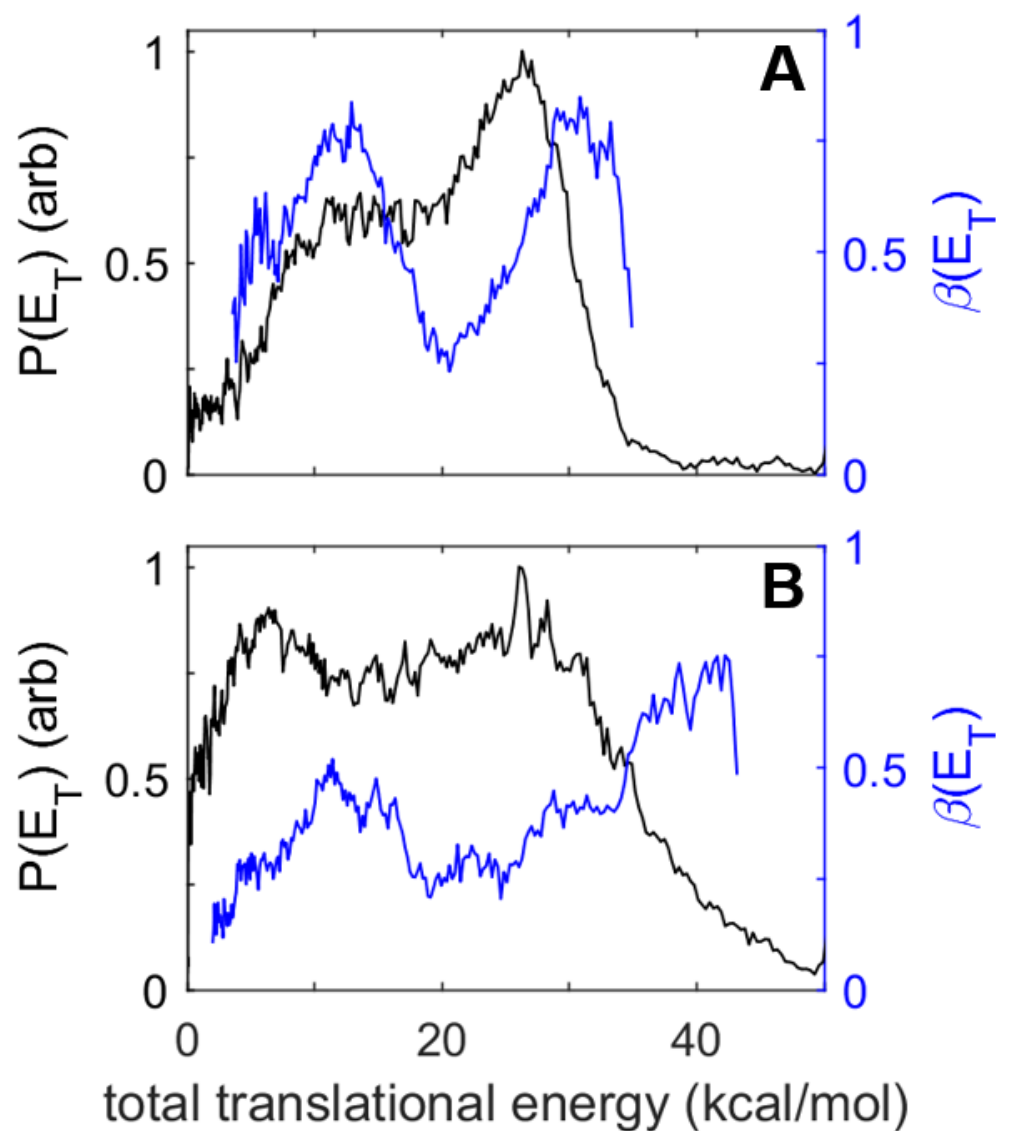

Fig. 4.3 Translational energy distributions (black) and anisotropy parameter $\beta$ (blue) of $\mathrm{Cl}$ from 1-color UV (A) and 2-color (B) photodissociation of propargyl chloride. 
allowed transition. The $\mathrm{Cl}^{*}$ angular distributions given in Fig. 4.4A and $\mathrm{B}$ also show a significant reduction in overall anisotropy. The peak associated with $T_{2}$ changes from $\beta \sim-0.4$ to 0.1 . The main peak associated with $T_{3}$ shows only a modest reduction in anisotropy, while again the new signal beyond $32 \mathrm{kcal} \mathrm{mol}^{-1}$ shows an increase in $\beta$ just as in the case of $\mathrm{Cl}$, again suggesting access to $S_{2}$ for these hot molecules.
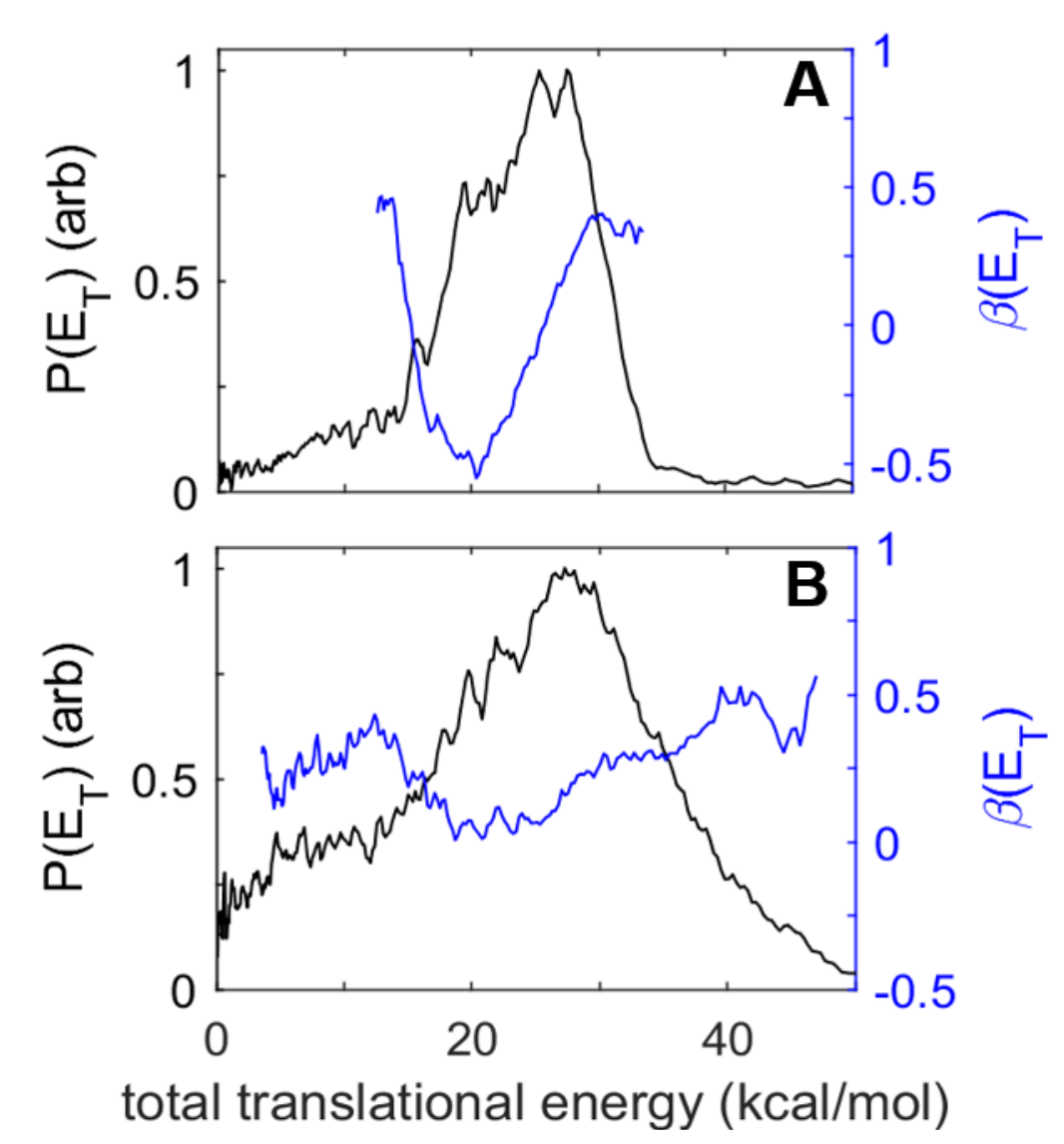

Fig. 4.4 Translational energy distributions (black) and anisotropy parameter $\beta$ (blue) of $\mathrm{Cl}^{*}$ from 1-color UV (A) and 2-color (B) experimental configurations of propargyl chloride photodissociation.

Initial excitation by intense IR light gives access to unexplored parts of the potential energy surfaces, both in IRMPD and IR-enhanced UV photodissociation. The key difference between the two is that for IRMPD, we exclusively study dissociation from the ground electronic state. Previously, we reported that UV photodissociation at $235 \mathrm{~nm}$ does not yield $\mathrm{HCl}$ elimination and ascribed this to processes involving largely repulsive excited states. With IRMPD, however, we 
find evidence that the $\mathrm{HCl}$ elimination channel opens, as we detect $\mathrm{HCl}$ state-selectively for the first time.

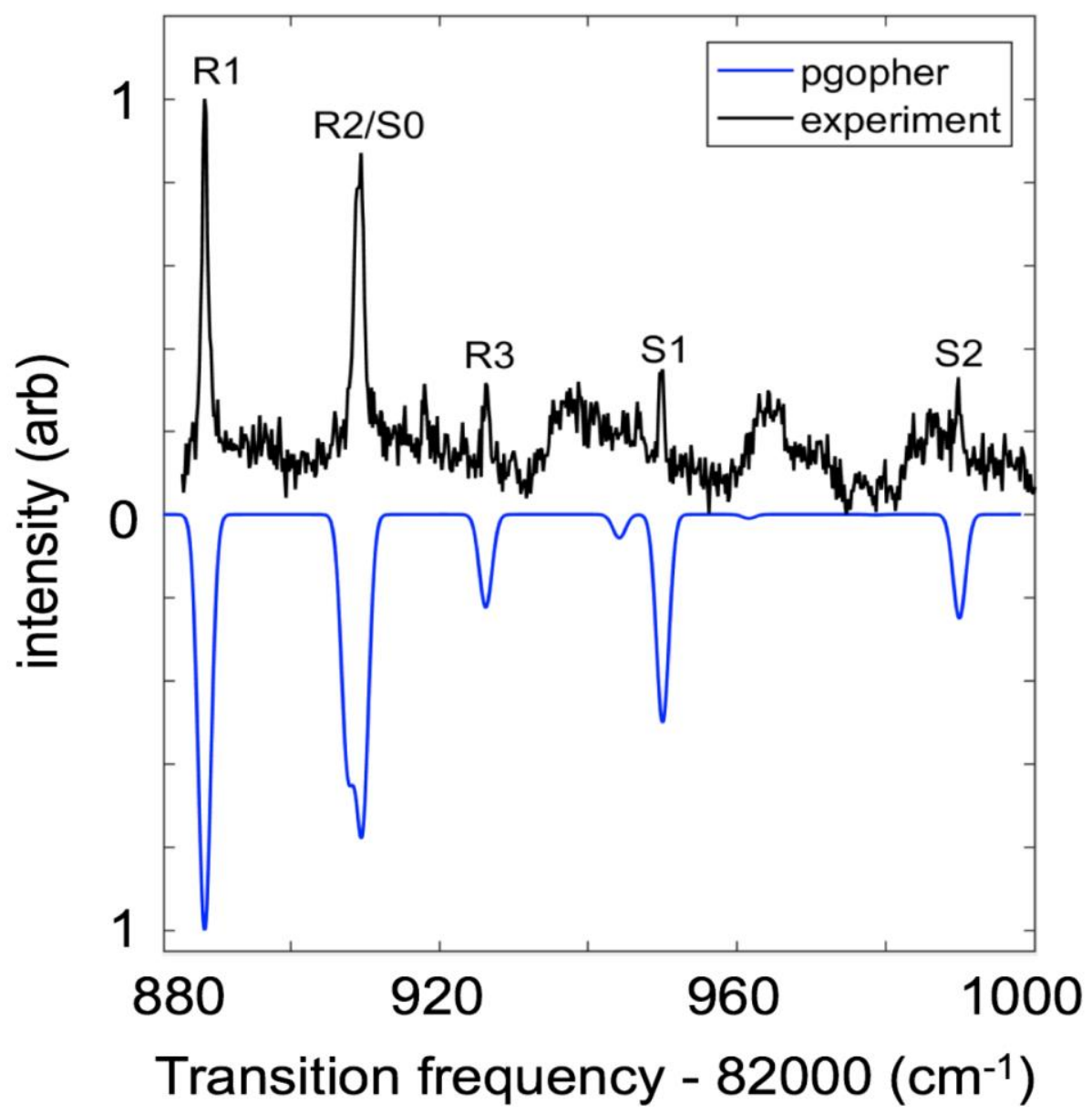

Fig. 4.5 Experimental HCl IRMPD REMPI spectrum (top trace) and simulation at 85 $\mathrm{K}$ (bottom trace).

The $\mathrm{HCl}$ rotational distribution was obtained by scanning the probe laser after the IR excitation. The experimental REMPI spectrum can be seen in the top trace of Fig. 4.5 with $\mathrm{HCl}$ products detected on the $\mathrm{S}$ and $\mathrm{R}$ branches of the $\mathrm{F}^{1} \Delta_{2} \mathrm{X}^{1} \Sigma^{+}(0,0)$ transition. Unfortunately, the spectrum is quite noisy owing to non-resonant background signals in which there is also some regular oscillation from etalon effects at a window. By modeling the relative abundance of the rotational population using PGOPHER (bottom trace Fig. 4.5), ${ }^{8,189}$ we inferred that $\mathrm{HCl}$ fragments were produced very cold with a rotational temperature of $\sim 85 \mathrm{~K}$. 
We have also recorded $\mathrm{HCl}$ images on several transitions and we show the results for $\mathrm{R}(1)$ in Fig. 4.6A. The image shows an intense central spot tapering to very low intensity that extends at considerable distance from the image center. Analysis of the image after subtraction of the background signal gives the distributions in Fig. 4.6B. The $\mathrm{P}\left(\mathrm{E}_{\mathrm{T}}\right)$ shows a peak at very low translational energy, near $1 \mathrm{kcal} \mathrm{mol}^{-1}$, dropping quickly to around $10 \mathrm{kcal} \mathrm{mol}^{-1}$, after which there is a tail out to $\sim 60 \mathrm{kcal} \mathrm{mol}^{-1}$. This clearly appears to consist of two components: one of these peaks at low energy typical of a ground state, barrierless dissociation, while the other encompasses

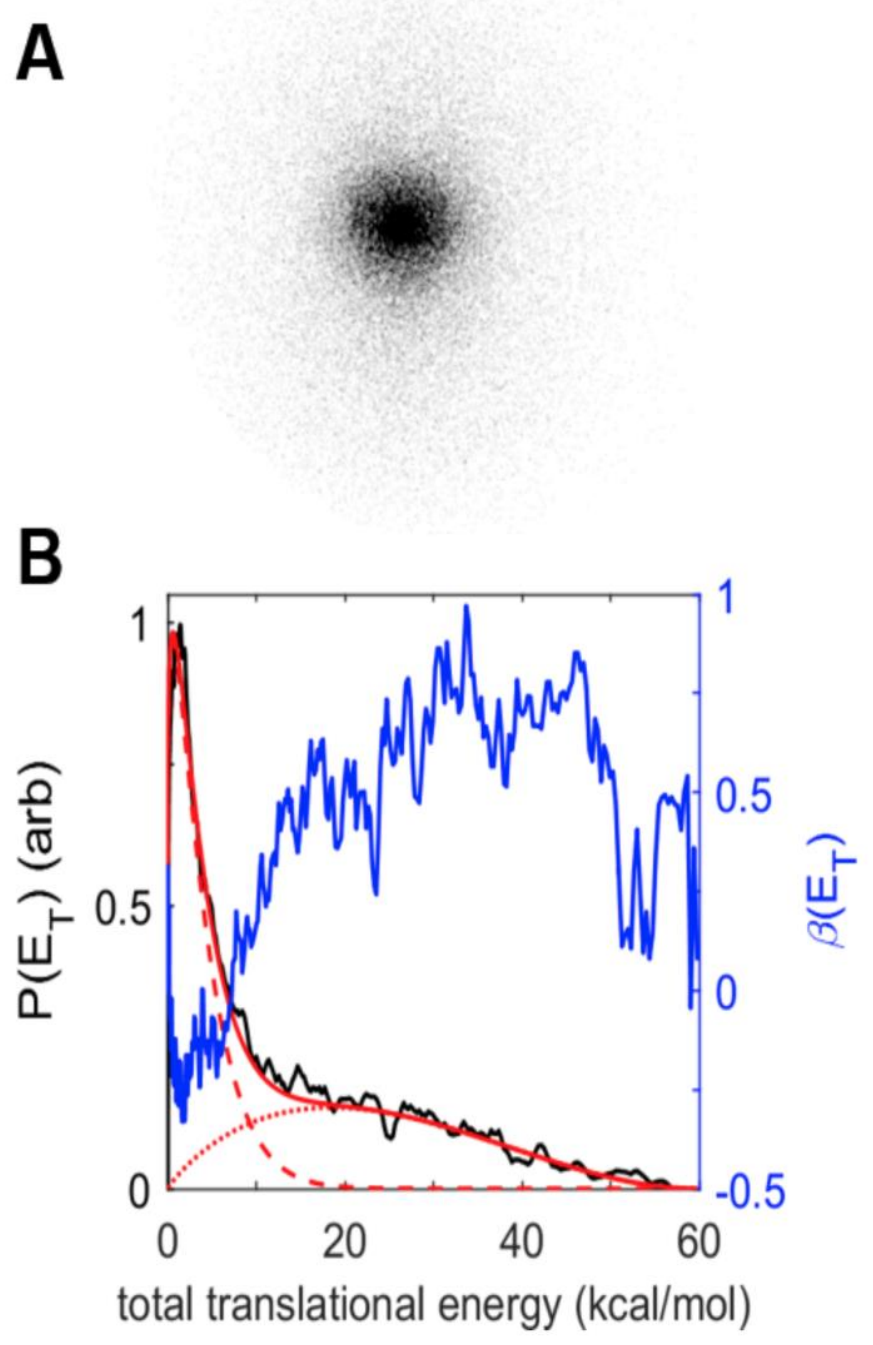

Fig. 4.6 DC slice image of $\mathrm{HCl}(\mathrm{v}=0, \mathrm{j}=1)(\mathrm{A})$ and translational energy distribution (black) and anisotropy parameter b (blue) (B) from 2-color photodissociation of propargyl chloride. Red lines show decomposition of the distribution to two components (see text). 
a broad distribution such as over a barrier or from an excited state process. We have fitted it to a sum of two distributions each using a flexible form that readily represents these two components:

$$
P\left(E_{T}\right)=a\left(\frac{E_{T}}{E_{\text {Tmax }}}\right)^{b}\left(\frac{1-E_{T}}{E_{T \max }}\right)^{c}
$$

and the result is shown in red in Fig. 4.6B. Also shown in Fig. 4.6B is the recoil-dependent angular distribution summarized as $\beta\left(\mathrm{E}_{\mathrm{T}}\right)$.

It is very illuminating to examine these considering results from Butler and coworkers at $193 \mathrm{~nm}$. They exerted considerable effort to distinguish the contributions of $\mathrm{C}_{3}{ }^{+}(\mathrm{m} / \mathrm{z}=36)$ from $\mathrm{C}_{3} \mathrm{H}_{3}$ fragmentation in the electron impact ionizer to the $\mathrm{HCl}^{+}$signal at the same mass-to-charge ratio. ${ }^{137}$ They concluded that the $\mathrm{HCl}$ elimination $\mathrm{P}\left(\mathrm{E}_{\mathrm{T}}\right)$ is bimodal with a minor peak at low translational energy and a broad one extending to $60 \mathrm{kcal} \mathrm{mol}^{-1}$ or so, much as we see here. However, their low energy peak was roughly ten-fold lower than what is seen here. We have argued above that initial vibrational excitation gains direct access to $S_{2}$, so it is reasonable to expect a component in our $\mathrm{HCl}$ distribution that also resembles the $193 \mathrm{~nm}$ result which was assigned to $\mathrm{S}_{2}$. We suggest that the slow component is ground state dissociation, mostly from direct IRMPD, but there may be a small contribution from internal conversion from $\mathrm{S}_{2}$ that would also account for the analogous peak in the $193 \mathrm{~nm}$ result. The broad fast component is then entirely due to IRenhanced UV dissociation through $\mathbf{S}_{2}$. This is confirmed by the angular distributions which are close to isotropic at low recoil energy but become predominantly parallel in the region of the dissociation attributed to $S_{2}$. One question that arises is how we could obtain a rotational temperature of $85 \mathrm{~K}$ given the broad component of the TEDs that extends to such high energy. To fit both we would clearly require two different rotational temperatures. Unfortunately, the high TEDs associated to the $S_{2}$ contribution is likely so Doppler broadened that it is lost in the 
considerable noise in the REMPI spectrum, and we only detect the intense features near line centers that are associated to the IRMPD.

In our previous study we examined the excited state potential surfaces in some detail. Here, to aid in understanding the IRMPD, we characterize stationary points on the ground electronic state. The relative ZPEs of all structures are shown in Fig. 4.7. The threshold for $\mathrm{Cl}$ elimination is calculated to be $69.8 \mathrm{kcal} \mathrm{mol}^{-1}$, in reasonable agreement with the value from the active thermochemical tables of $68.6 \mathrm{kcal} \mathrm{mol}^{-1} .{ }^{1,156}$ Four different pathways have been identified for $\mathrm{HCl}$ elimination to aid in understanding the dynamics of that process. Two of these lead to formation of linear propadienylidene $\left(1-\mathrm{C}_{3} \mathrm{H}_{2}\right)$ and another pathway gives cyclic propadienylidene (c- $\mathrm{C}_{3} \mathrm{H}_{2}$ ) as the co-product of $\mathrm{HCl}$, while a fourth gives singlet propargylene. The barrier for elimination giving the cyclic product is prohibitively high at $127 \mathrm{kcal} \mathrm{mol}^{-1}$. There is a pathway for direct $\mathrm{HCl}$ elimination from propargyl chloride giving $1-\mathrm{C}_{3} \mathrm{H}_{2}$ with a barrier of $103 \mathrm{kcal} \mathrm{mol}^{-1}$. Direct $\mathrm{HCl}$ loss to form singlet propargylene, $\mathrm{HCCCH}$, is also possible as shown. We were unable to locate a transition state for this and conclude it is barrierless and endoergic by $88 \mathrm{kcal} \mathrm{mol}^{-1}$.

Alternatively, isomerization to 1-chloroallene has a barrier of only $58.8 \mathrm{kcal} \mathrm{mol}^{-1}$, after which there is dissociation at $66 \mathrm{kcal} \mathrm{mol}^{-1}$. This is the lowest energy decomposition pathway. In this pathway two transition states are found: one corresponding to the isomerization to 1chloroallene and the other to $\mathrm{HCl}$ formation from 1-chloroallene leading to a van der Waals well. The second transition state separating 1-chloroallene and the well, highlighted in Fig. 4.7 has the $\mathrm{Cl}$ atom in an abstraction geometry rather than a 3-center or 4-center $\mathrm{HCl}$ elimination structure. This is much as what we have seen for $\mathrm{HCl}$ elimination in $\mathrm{Cl}+$ butene reactions, for which only TSs of roaming character connect the strongly bound adducts to the elimination of $\mathrm{HCl}$ at the allylic sites. ${ }^{190,191}$ 
That is, the $\mathrm{Cl}$ atom must come off of 1-chloroallene to attain the abstraction geometry. This view is supported by the energy of the TS that is near that of the dissociated radical pair, as well as its low vibrational frequencies: the TS has an imaginary frequency of $-193.35 \mathrm{~cm}^{-1}$ and two low bound frequencies of 72.76 and $196.20 \mathrm{~cm}^{-1}$.

To support our identification of the $\mathrm{C}_{3} \mathrm{H}_{2}$ structure, we examined the $193 \mathrm{~nm}$ photodissociation of propargyl chloride in our CPUF apparatus, and the results are shown in Fig. 4.8. Although this is a different wavelength from that employed in the imaging study above, in both cases we believe the $\mathrm{HCl}$ results from dissociation on the ground state, so we expect the same product isomer. Results for propargyl chloride are presented along with those for 1,2-butadiene that were previously studied to understand branching in propargyl radical dissociation. ${ }^{183}$ These experiments were performed in a quasi-uniform flow at low temperature but in a collisional environment to permit relaxation of the products to the local temperature $(\sim 14 \mathrm{~K})$ for detection. In the previous study, propargyl radical was first prepared by photodissociation of 1,2-butadiene. Absorption of a second photon gives the radical products which are then cooled in the flow and detected. In Fig. 4.8A we show representative raw signals on the indicated rotational transitions for $1-\mathrm{C}_{3} \mathrm{H}_{2}$ and $\mathrm{c}-\mathrm{C}_{3} \mathrm{H}_{2}$ but additional transitions are included and averaged (after normalizing by the density and weighting by the linestrength) to give the branching, $1-\mathrm{C}_{3} \mathrm{H}_{2} /\left(1-\mathrm{C}_{3} \mathrm{H}_{2}+\mathrm{c}-\mathrm{C}_{3} \mathrm{H}_{2}\right)$, shown in Fig. 4.8B. The red spectra indicate times in the flow we associate with nascent photochemical products after cooling to the flow temperature, 60-70 ms after the photolysis laser. We attribute the intensity and time-dependence of $\mathrm{C}_{3} \mathrm{H}_{2}$ signals from 1,2-butadiene exclusively to $\mathrm{H}$ atom loss from propargyl radical, with negligible contribution of $\mathrm{CH}_{4}$ loss from the parent molecule. Also shown in Fig. 4.8 is the analogous result for propargyl chloride photodissociation. Although propargyl chloride does 


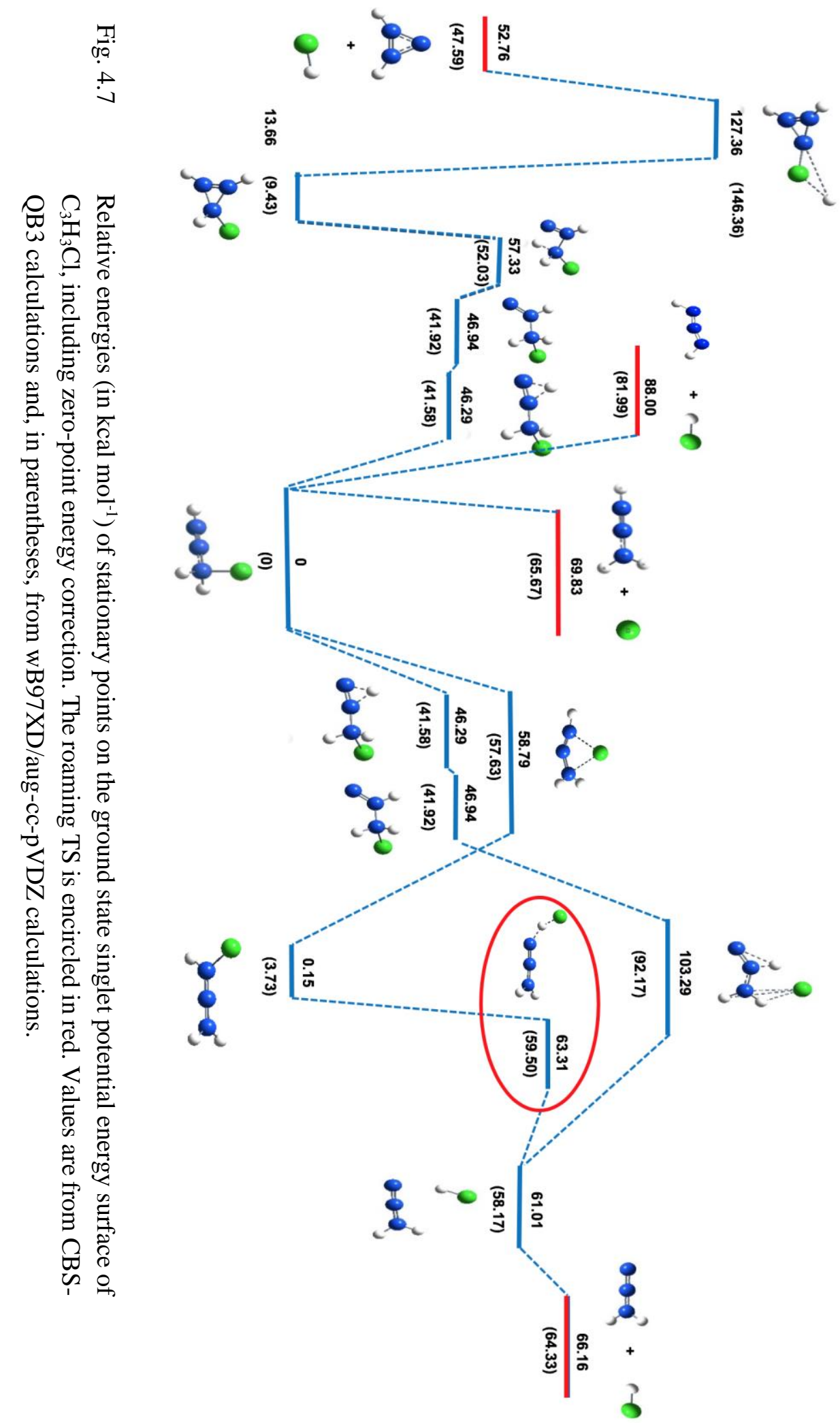


produce propargyl radical that can subsequently undergo the same chemistry, $\mathrm{C}_{3} \mathrm{H}_{2}$ products are also clearly formed directly by $\mathrm{HCl}$ loss from the propargyl chloride parent as discussed extensively in the literature. Thus, the two precursors give rise to different branching fractions between the $\mathrm{C}_{3} \mathrm{H}_{2}$ isomers. In addition to $\mathrm{H}$ atom loss from the propargyl radical that occurs for both propargyl chloride and 1,2-butadiene, for the former there is also direct $\mathrm{HCl}$ elimination. The increased branching to linear over cyclic- $\mathrm{C}_{3} \mathrm{H}_{2}$ seen in Fig. 4.8 shows that $1-\mathrm{C}_{3} \mathrm{H}_{2}$ is the dominant isomer formed following $\mathrm{HCl}$ elimination from propargyl chloride. Subsequent disappearance of 1- $\mathrm{C}_{3} \mathrm{H}_{2}$ and appearance of intense $c-\mathrm{C}_{3} \mathrm{H}_{2}$ (after $70 \mu \mathrm{s}$ ) is ascribed to $\mathrm{H}$-atom catalyzed isomerization in the high-density region of the flow as discussed previously. ${ }^{183}$

Singlet propargylene is also a possible product isomer, but we cannot detect it owing to its negligible dipole moment. We would expect this to undergo $\mathrm{H}$ catalyzed isomerization to $\mathrm{c}-\mathrm{C}_{3} \mathrm{H}_{2}$, which would appear at later times, just as we have shown for the triplet propargylene and $1-\mathrm{C}_{3} \mathrm{H}_{2}$. Much less of the late $\mathrm{c}-\mathrm{C}_{3} \mathrm{H}_{2}$ from $\mathrm{H}$-catalyzed isomerization is observed in the case of propargyl chloride. This may reflect secondary chemistry involving $\mathrm{Cl}$ atoms, but in any case, it does not suggest the presence of a distinct dark channel. The much higher energy onset of $\mathrm{HCCCH}$ also suggests it would be weak or negligible relative to the $\mathrm{HCl}$ loss from chloroallene under conditions of IRMPD. Although we can readily determine the branching between the different isomers detected, we do not know the probability of direct propargyl chloride photodissociation vs. absorption of a second photon by the propargyl radical, so we cannot determine the branching for $\mathrm{HCl}$ elimination from this data. In short, these rotational spectra for propargyl chloride photodissociation at $193 \mathrm{~nm}$ show enhanced formation of $1-\mathrm{C}_{3} \mathrm{H}_{2}$ compared to 1,2-butadiene, supporting its assignment as the product isomer as identified in the ab initio calculations. 


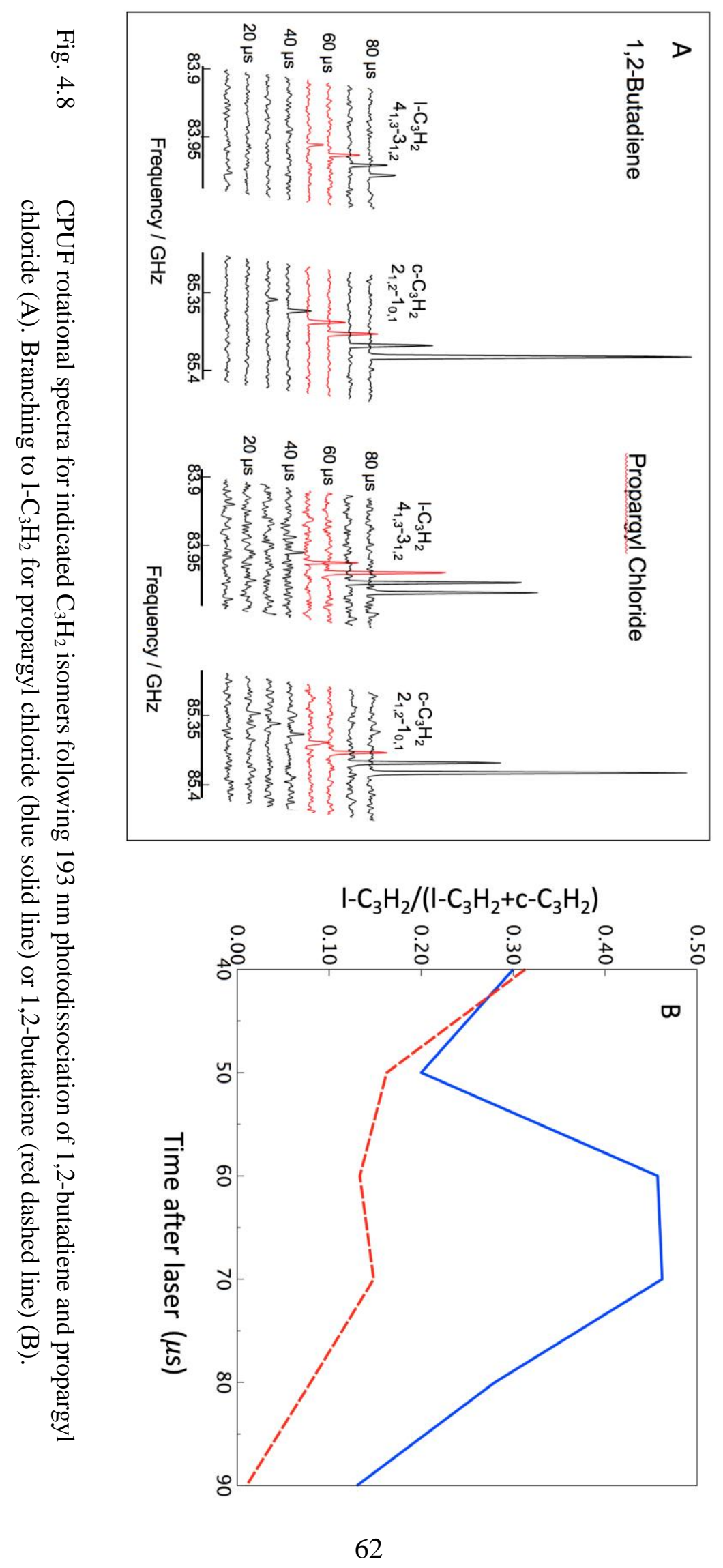




\subsection{Conclusion}

TEDs of $\mathrm{Cl}$ fragments reveal dissociation from multiple pathways, including UV, IRenhanced UV, and IRMPD processes in these experiments. IRMPE results in the enhancement of UV photodissociation processes and additional fast fragments and parallel anisotropy which we attribute to access to $\mathrm{S}_{2}$ from distorted geometries. IRMPD produces a high abundance of slow $\mathrm{Cl}$ fragments and less slow $\mathrm{Cl}^{*}$ fragments. We interpret these fragments peaking at low energy to be the result of dissociation near threshold from the ground state. $\mathrm{HCl}$ is likely formed both by IRMPD, peaking at low energy, and from $\mathrm{S}_{2}$ extending to $60 \mathrm{kcal} \mathrm{mol}^{-1}$. A quantum chemical survey of the ground state PES indicates that the lowest energy decomposition pathway involves isomerization to 1-chloroallene followed by a roaming-like transition state that assumes an abstraction geometry to produce $\mathrm{HCl}$ and $1-\mathrm{C}_{3} \mathrm{H}_{2}$. This is in line with the cold rotational temperature measured for the low energy peak. CPUF experiments, although performed at 193 $\mathrm{nm}$, further support the production of $1-\mathrm{C}_{3} \mathrm{H}_{2}$ in photodissociation of propargyl chloride. Results presented here combined with our recent characterization of its UV photodissociation provide an improved understanding of the potential energy landscape and associated dissociation dynamics of propargyl chloride. 


\section{CHAPTER 5}

\section{HDCO SPECTROSCOPY AND RADICAL DISSOCIATION THRESHOLDS}

\subsection{Introduction}

Formaldehyde has been vital to advancements in spectroscopy and the understanding of polyatomic dissociation dynamics. ${ }^{24,30,31,44,170,192-202}$ The most well-studied four atom molecule, it is tractable theoretically and easily studied via absorption of frequency doubled light from YAGpumped dye lasers for electronic spectroscopy. ${ }^{51,194,203-205}$ Infrared and microwave radiation have also been used for thorough vibrational and rotational investigations, respectively. ${ }^{206-214}$ Accessible and long-lived singlet and triplet excited states permit state-specific excitation, and radical and molecular dissociation pathways in formaldehyde make it a perfect prototype system to develop an understanding of various dynamical events including characterization of the roaming mechanism. ${ }^{24,43,44} \mathrm{We}$ are interested in investigation of quantum effects in roaming, and ZPE differences for isotopologues represent a singular quantum effect whose impact on roaming have yet to be explored. In this chapter we present an initial determination of the H- and D- loss thresholds in HDCO, which in turn allows for a precise determination of the heats of formation of HDCO and DCO. While different symmetry and ZPE are different for $\mathrm{HDCO}$ and $\mathrm{H}_{2} \mathrm{CO}$, energy flow dynamics are analogous. A schematic of potential energy curves and relevant energies important to formaldehyde spectroscopy and dynamics is shown in Fig. 5.1. ${ }^{53}$

Investigation of the isotopologues $\mathrm{D}_{2} \mathrm{CO}$ and $\mathrm{HDCO}$ and comparison to $\mathrm{H}_{2} \mathrm{CO}$ has improved spectroscopic theory and provided general insight into molecular dissociation dynamics. ${ }^{215-219}$ Formaldehyde is also one of the most abundant molecules in interstellar space and HDCO has been detected in OMC-1, cometary objects, and protostars. ${ }^{20,221}$ Observation of 


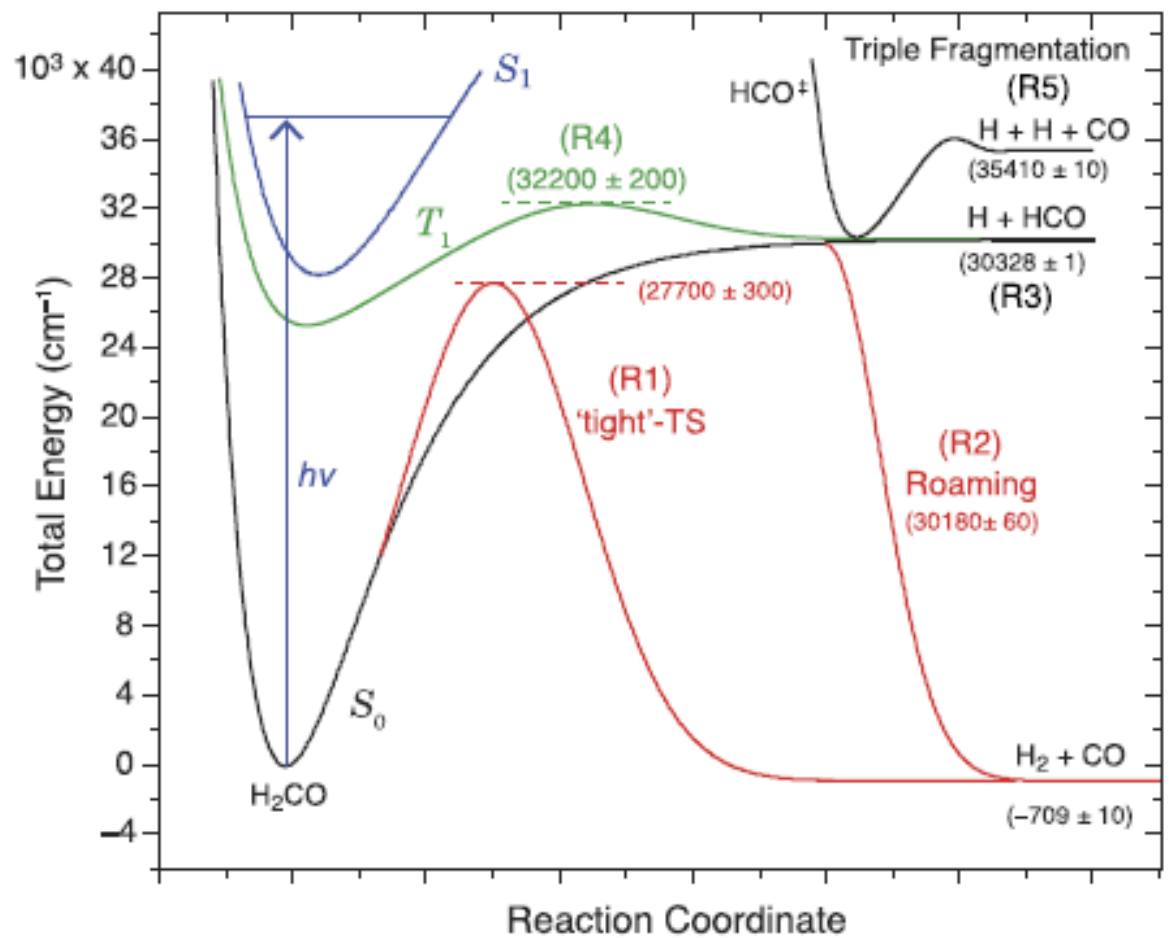

Fig. 5.1 Schematic of relevant PES cuts with energies and pathways indicated. Adapted with permission from M.S. Quinn et al, J. Chem. Phys., 147, 013935 (2017) (C) AIP.

deuterated species may help determine the physical and chemical conditions of star-forming regions. ${ }^{20,222-224}$ Extensive rotational analyses have been performed by microwave and millimeter wave methods, including a study using several spectrometers and older measurements compared to ab initio predictions. ${ }^{208}$ Infrared and Raman spectra of HDCO were first reported by Davidson, Stoicheff, and Bernstein. ${ }^{211}$ The ground vibrational state was further characterized through analysis of rotational fine structure by Job, Sethuraman, and Innes, ${ }^{216} v_{3}, v_{5}$ and $v_{6}$ were investigated by Johns and McKellar using conventional infrared absorption and CO laser Stark spectroscopy, ${ }^{209,210}$ and finally, all fundamentals were updated and summarized by Clouthier and co-workers. ${ }^{197}$ Large scale variational calculations were also performed to determine the vibrational structure and mixing, ${ }^{206,207}$ and higher resolution measurements have improved agreement between theoretical and experimental line intensities and positions in addition to new line lists over a broader frequency range. $^{225}$ 
In addition to ground state characterization, Job and co-workers provided a thorough vibrational and rotational analysis of the ${ }^{1} \mathrm{~A}_{2}-{ }^{1} \mathrm{~A}_{1}$ system, ${ }^{216}$ including determination of excited state fundamentals. Bands of this system were later reinvestigated with higher resolution and magnetic rotation, ${ }^{226}$ leading to a reassignment of $v_{3}$ to $v_{6}$ and deduction of a mechanism for selection rules. A similar vibrational and rotational analysis of the ${ }^{3} \mathrm{~A}_{2}-{ }^{1} \mathrm{~A}_{1}$ system for four vibronic bands, as was done for ${ }^{1} \mathrm{~A}_{2}-{ }^{1} \mathrm{~A}_{1}$, determined the triplet geometry and identified Coriolis perturbations. ${ }^{227}$ Normal modes of vibration and symmetry species of $\mathrm{H}_{2} \mathrm{CO}$, analogous to $\mathrm{HDCO}$, are included in Fig. 5.2. ${ }^{197,228}$ Designation of inertial axes in cartesian coordinates are also indicated.
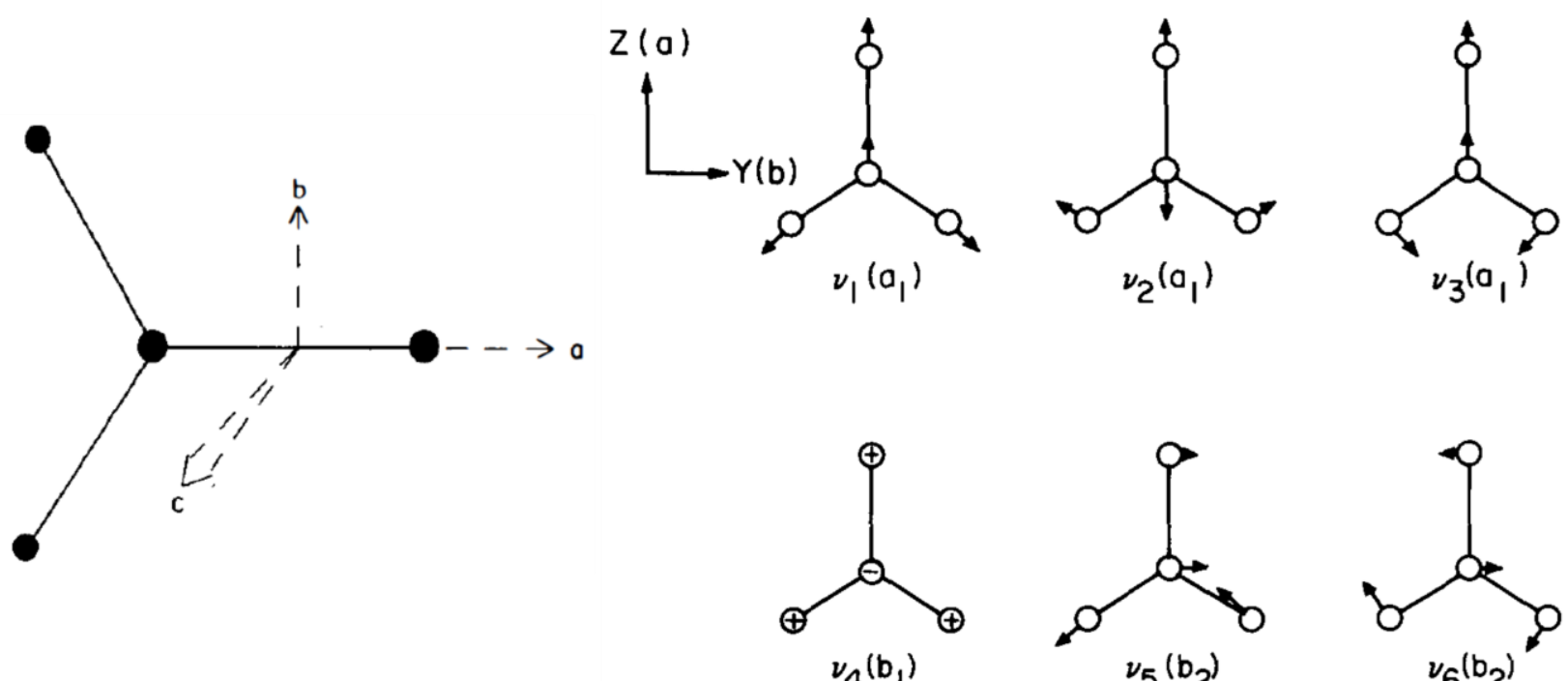

Fig. 5.2

Left. Inertial axis designations. Right. Normal modes of vibration and symmetry species. Adapted with permission from D.J. Clouthier et al, Ann. Rev. Phys. Chem., 34, 31-58 (1983) (C) Annual Reviews and D.E. Reisner et al, J. Chem. Phys., 80(12), 5968-5978 (1983) @ AIP.

Deuterium substitution results in longer fluorescence decay times and causes the $\mathrm{CO}$ rotational distribution from the tight transition state pathway to be shifted higher. ${ }^{218,219,229,230}$ Shorter lifetimes in $\mathrm{H}_{2} \mathrm{CO}$ are ascribed to $\mathrm{S}_{1}$ coupling to the $\mathrm{S}_{0}$ "lumpy continuum" where ground state vibrational levels are broadened by tunneling through the barrier to molecular products. ${ }^{194,201,218,220}$ A higher CO rotational distribution can be explained using the rotational 
reflection principle, where the maximum $j_{\mathrm{CO}}$ is scaled with the square root of the reduced mass of deuterium substitution of $\mathrm{H}_{2} \cdot{ }^{215,231}$ A comprehensive study of $\mathrm{H}_{2} \mathrm{CO}, \mathrm{HDCO}$, and $\mathrm{D}_{2} \mathrm{CO}$ using photoacoustic absorption and VUV laser-induced fluorescence detection of atomic products then determined $\mathrm{T}_{1}$ barrier height, the $\mathrm{S}_{1}-\mathrm{T}_{1}$ intersystem crossing rate, and radical dissociation thresholds. ${ }^{232}$ Threshold energies were calculated assuming a Born-Oppenheimer potential, and threshold differences arise from ZPE differences. For $\mathrm{H}_{2} \mathrm{CO}$ and $\mathrm{D}_{2} \mathrm{CO}$, lower bounds were determined experimentally by comparing photoacoustic and PHOFEX spectra and upper bounds were set at the origin of the vibrational band where the PHOFEX and photoacoustic spectra match completely.

Decades of experimental and theoretical work have since been able to determine the radical threshold for $\mathrm{H}_{2} \mathrm{CO}, 30327.6 \pm 0.9 \mathrm{~cm}^{-1}$, accurately. ${ }^{2,215,233}$ The same cannot be said for HDCO. For HDCO, only the D atom radical threshold has been obtained, derived from an extrapolation of the H/D branching ratio to infinity. ${ }^{232}$ The experimental and calculated thresholds reported have a $\sim 100 \mathrm{~cm}^{-1}$ uncertainty and have not been updated for more than three decades. Using $\mathrm{H}$ and D atom REMPI detection and velocity map imaging, ${ }^{101,103}$ translational energy determination of atomic fragments at a range of energies allows for a more accurate determination of HDCO ground state radical thresholds with little uncertainty.

\subsection{Experimental Methods}

Synthesis of paraformaldehyde-d1 was adapted from a previously described method, which is detailed below. ${ }^{234} \mathrm{CDBr}_{3}(50 \mathrm{~g})$ was added to an oven-dried $200 \mathrm{ml}$ round bottom flask, attached to a reflux condenser and an argon-filled balloon, and then Bu3SnH (57 g) was added dropwise carefully. After addition and waiting for $10 \mathrm{~min}$, the reaction mixture was heated to reflux for 25 
h. The mixture then was distilled to give pure $\mathrm{CDHBr}_{2} \cdot \mathrm{CDHBr}_{2}(15 \mathrm{~g})$ was placed in a $200 \mathrm{~mL}$ round bottom flask. Glacial acetic acid $(54 \mathrm{ml})$ and acetic anhydride $(6 \mathrm{ml})$ were added. Potassium acetate (26 g) was then added in one portion and the reflux condenser and an argon-filled balloon were quickly attached to the round bottom flask. The reaction mixture was heated at reflux for 40 h. The mixture was then allowed to cool to room temperature and $150 \mathrm{ml}$ of diethyl ether were added. White precipitated potassium bromide was filtered off several times and more diethyl ether was added until there was no precipitated potassium bromide formed. The filtrates were combined, and the solvent was removed by a rotary evaporator to afford the pure d-methylene diacetate in $78 \%$ yield. d-Methylene diacetate $(9 \mathrm{~g})$ was placed in a $25 \mathrm{~mL}$ round bottom flask and HPLC grade water $(1 \mathrm{ml})$ and conc. $\mathrm{HCl}(1 \mathrm{ml})$ were added. The reaction mixture was then heated to reflux for $24 \mathrm{~h}$. The solvent was removed by oil pump and paraformaldehyde-d 1 was formed as a white powder.

HDCO monomers were produced by a method adapted from previous experiments in which monomers are liberated from paraformaldehyde-d1 upon heating. ${ }^{233}$ Paraformaldehyde-d1, anhydrous $\mathrm{MgSO}_{4}$, and glass wool were packed into a $\mathrm{KF}$ elbow before the valve. The $\mathrm{KF}$ elbow, valve, and connecting stainless steel parts were wrapped with braided fiberglass heating cord and heated to $\sim 70^{\circ} \mathrm{C}$ to liberate $\mathrm{HDCO}$ monomers from paraformaldehyde-d1. A 930 Torr helium backing pressure resulted in a $\sim 5 \%$ formaldehyde concentration and a beam temperature of $\sim 18$ K. Beam temperature was approximated from relative populations of HDCO rovibrational lines and known experimental conditions of $\mathrm{H}_{2} \mathrm{CO}$ experiments also being performed.

UV light was generated by frequency doubled or tripled narrow linewidth $\left(0.1 \mathrm{~cm}^{-1}\right)$ dye lasers pumped by Nd:YAG lasers. Pump energies were less than $\sim 0.2 \mathrm{~mJ} /$ pulse and probe energies were $\sim 0.2 \mathrm{~mJ} /$ pulse. PHOFEX spectra were obtained by scanning the excitation/dissociation laser 
from 30,100 to $31,800 \mathrm{~cm}^{-1}$ for rovibrational bands on $S_{1}$ and using REMPI detection of $\mathrm{j}_{\mathrm{CO}}=44$ via $\mathrm{Q}\left(\mathrm{B}^{1} \Sigma^{+} \leftarrow \mathrm{X}^{1} \Sigma^{+}\right) .{ }^{106}$ Spectral simulations were done using $\mathrm{PGOPHER}^{8}$ and a fit was performed to assign rotational structure in vibrational bands of the experimental spectra. HDCO was then photodissociated with different pump frequencies corresponding to different rovibrational lines. HDCO ground and excited state $\mathrm{J}$ were between 0 and 4 , with a rotational energy typically around $6 \mathrm{~cm}^{-1}$ and up to $26 \mathrm{~cm}^{-1} . \mathrm{H}$ and $\mathrm{D}$ atoms were detected by $2+1$ REMPI via $2 \mathrm{~s}\left({ }^{2} \mathrm{~S}_{1 / 2}\right) \leftarrow 1 \mathrm{~s}\left({ }^{2} \mathrm{~S}_{1 / 2}\right)$ near $243 \mathrm{~nm} \cdot{ }^{3,110,235,236}$

\subsection{Results and Discussion}

Excitation energies were determined by first obtaining PHOFEX spectra of HDCO over a wide range. CO REMPI was used as the probe because molecular dissociation occurs well below both radical thresholds via a tight transition state pathway and this way background from $\mathrm{H}$ or $\mathrm{D}$ atoms from formyl radical multiphoton dissociation can also be avoided. A dense PHOFEX spectrum with more than twenty vibrational bands was recorded, Fig. 5.3. The spectral density is further demonstrated over a $250 \mathrm{~cm}^{-1}$ range, Figure 5.3 inset, with at least three partially overlapped vibrational bands, $2^{2} 6^{1}, 2^{2} 4^{3}$, and $2^{2} 4^{2} 6^{1}$. Green dots above the transitions indicate the rotational lines used to prepare $\mathrm{HDCO}$ on $\mathrm{S}_{1}$ in the imaging experiments. The radical thresholds determined in this work are also marked in Fig. 5.5.

Upon imaging $\mathrm{H}$ and $\mathrm{D}$ atom photoproducts using four different pump energies (Fig. 5.4 left and right columns, respectively), features with radii that increase with energy are observed. The top image is recorded on the rovibrational line where $\mathrm{H}$ and D atoms were first detected, indicated by the small center spot. The three subsequent images have pump energies increased by hundreds of wavenumbers. The increase in the maximum radius at which ions are observed with pump energy reflects the excess of the pump energy above the radical threshold. 


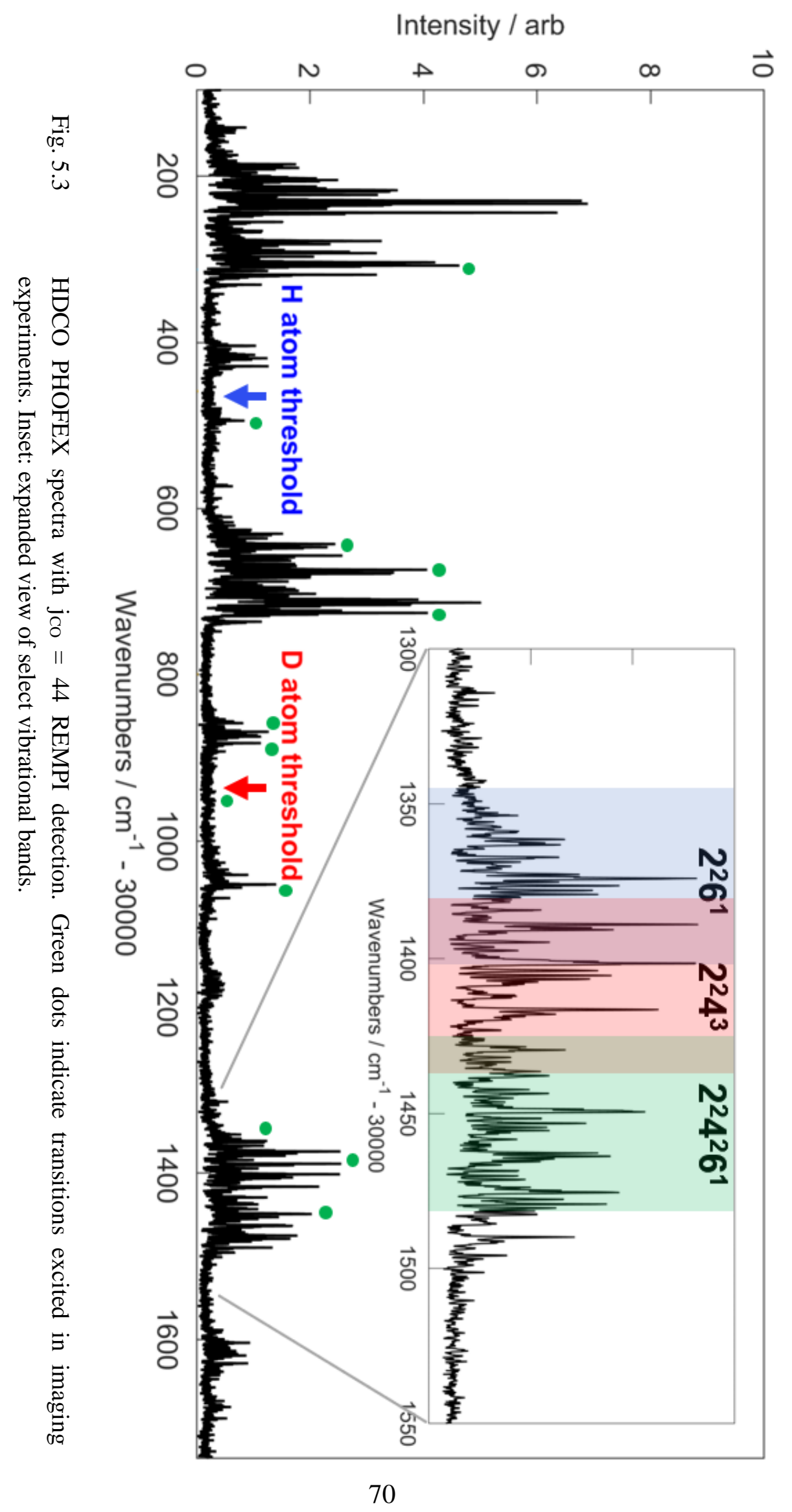


The intercept found in a plot of the translational energy vs. the upper state energy of the transition, Fig. 5.5, allows for the radical threshold energy to be determined more accurately than simply using the lowest energy rotational line that yielded atomic fragments. $\mathrm{H}$ and $\mathrm{D}$ atom thresholds of $30448 \pm 5$ and $30949 \pm 3 \mathrm{~cm}^{-1}$ were thus determined. Using the latter value with the heats of formation of D and HCO from the Active Thermochemical Tables, ${ }^{1,156}$ we obtain $\Delta \mathrm{H}_{0}{ }^{\mathrm{f}}(0$ $\mathrm{K})=-109.03 \pm 0.10 \mathrm{~kJ} / \mathrm{mol}$ for HDCO. Using this with the $\mathrm{H}$ atom threshold we obtain $\Delta \mathrm{H}_{0}{ }^{\mathrm{f}}(0 \mathrm{~K})$ $=39.17 \pm 0.14 \mathrm{~kJ} / \mathrm{mol}$ for DCO.

D atom TEDs, Fig. 5.6, for three of the images in Fig. 5.4 show the expected behavior, with increased pump energy directly correlating with increased translational energy. H atom TEDs, Fig. 5.7, for three of the images in Fig. 5.4, are not broad and structureless like the D atom $\mathrm{P}(\mathrm{E})$. The $\mathrm{H}$ atom image from the $31449.3 \mathrm{~cm}^{-1}$ excitation also exhibits a less intense faster distribution of fragments around an intense center spot, indicating concomitant excitation of the DCO bend.

Many $\mathrm{S}_{1}$ vibrational bands have previously been observed, and ${ }^{1} \mathrm{~A}_{2}-{ }^{1} \mathrm{~A}_{1}$ rovibrational lines assigned. Only select frequency ranges, however, have been assigned, likely due to spectral complexity and the relatively low resolution of previous measurements. Here, we have recorded PHOFEX spectra, Fig. 5.3, over an entire region at energies spanning nearly $2000 \mathrm{~cm}^{-1}$. The density of rovibrational lines and overlapping vibrational bands is exhibited by different colored boxes highlighting three different vibrational bands in Fig. 5.3 inset. A detailed analysis and assignment of these vibrational bands will be reported later.

Previously published calculated and experimental thresholds (often calculated for the different isotopologues using vibrational frequencies to predict the difference) were at least $35 \mathrm{~cm}^{-}$ ${ }^{1}$ lower than $\mathrm{H}$ and D atom thresholds of $30448 \pm 5$ and $30949 \pm 3 \mathrm{~cm}^{-1}$ determined here. A linear 


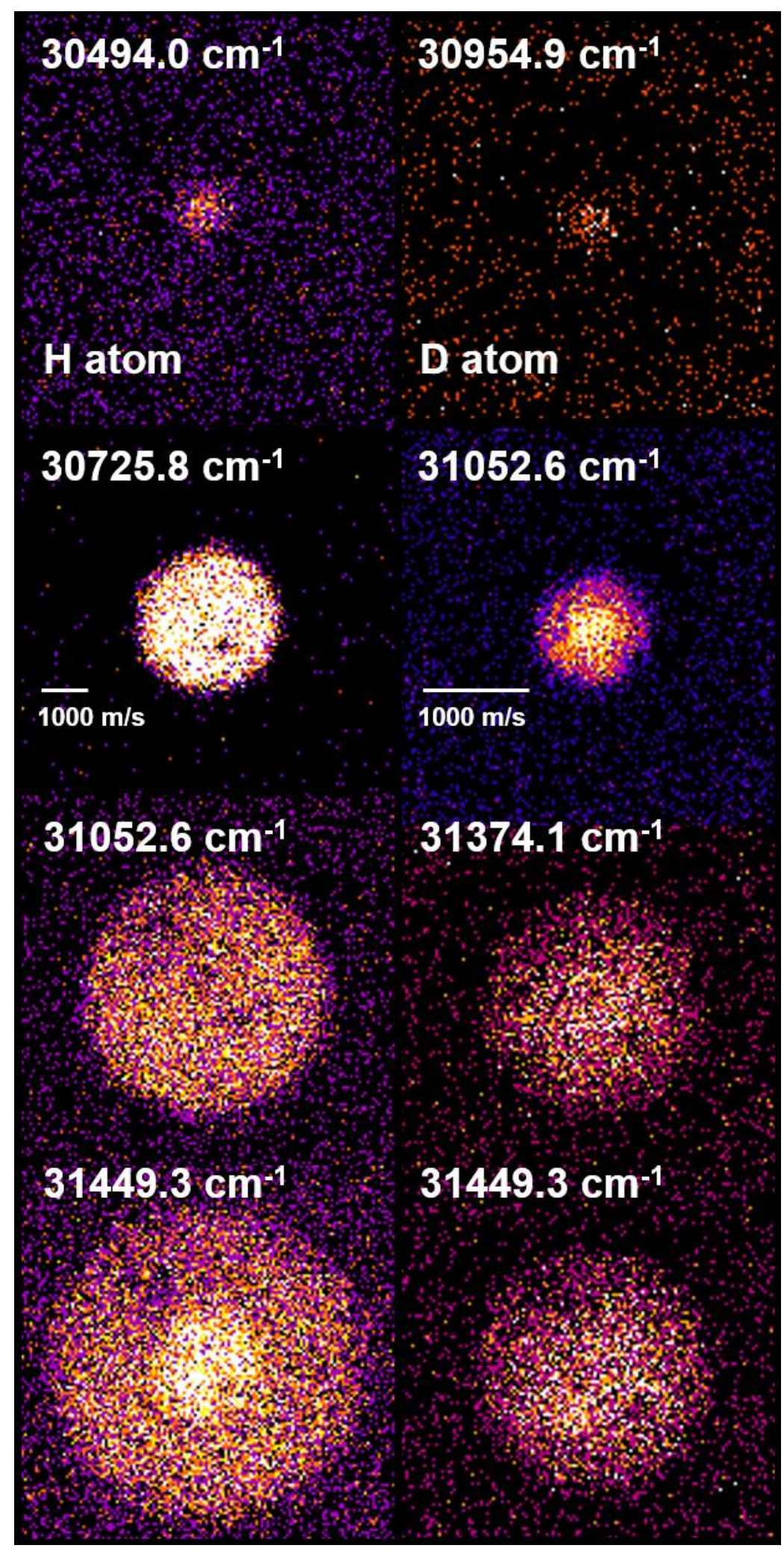

Fig. 5.4 Selected images of $\mathrm{H}$ atom (left column) and $\mathrm{D}$ atom fragments (right column) from excitation on different rovibrational lines of HDCO. 
least squares analysis was performed to determine the intercepts and the standard error of the dependent variable was used to determine the uncertainty. Uncertainty in these thresholds arises principally from the determination of the radius. Radii of images were determined by averaging the radius obtained in each quadrant separately, resulting in a standard deviation between 4 and 30 $\mathrm{cm}^{-1}$ for each point. Larger standard deviations at higher energies reflected in the error bars of Fig. 5.5 that contain two standard deviations, are a consequence of uniform uncertainty in image radii giving a quadratic dependence of the uncertainty in energy.

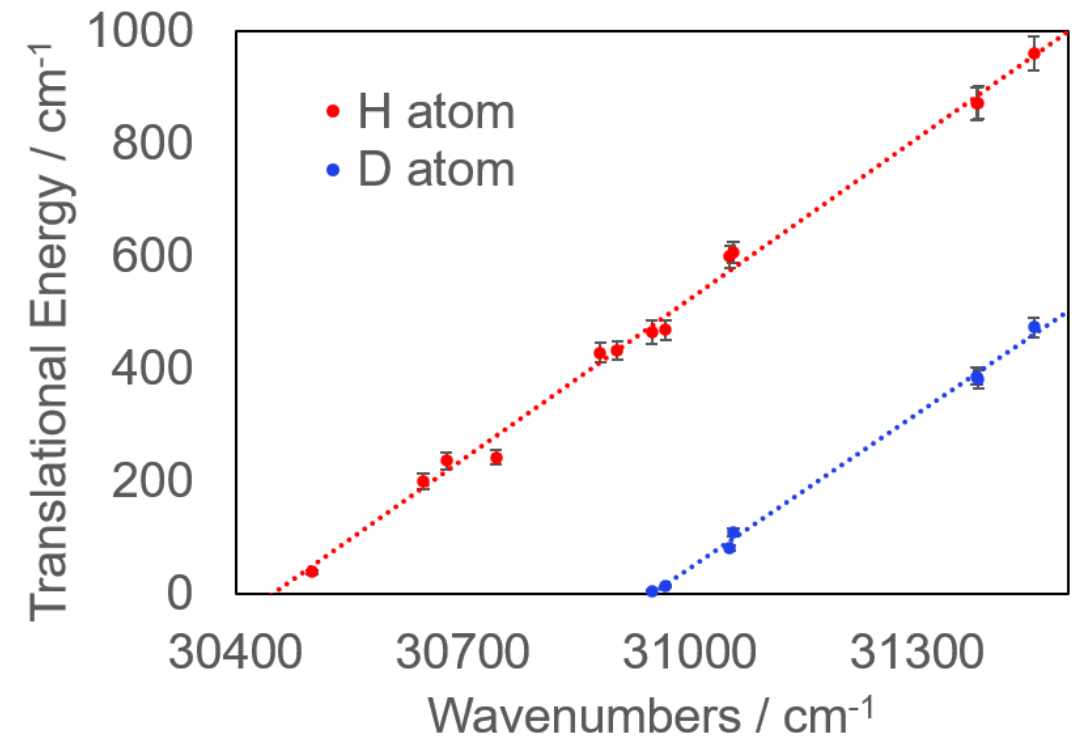

Fig. 5.5 Translational energy plotted against upper state energy for H atom (red) and D atom (blue) fragments.

This study offers the first reported experimental value for the $\mathrm{H}$ atom ground state radical threshold for HDCO. As for the D atom threshold, we note that at a pump energy of $30885.6 \mathrm{~cm}^{-1}$, an energy near the upper bound of the measured and calculated threshold range previously reported, D atoms were no longer detected. The previous experimental determination of the D atom threshold derives from a rough extrapolation of the H/D branching ratio. ${ }^{232}$ While the new value lies above the upper limit of the range determined in that study, the measurement here of 
translational energy release from a range of excitation energies has the advantage of allowing for a direct determination.

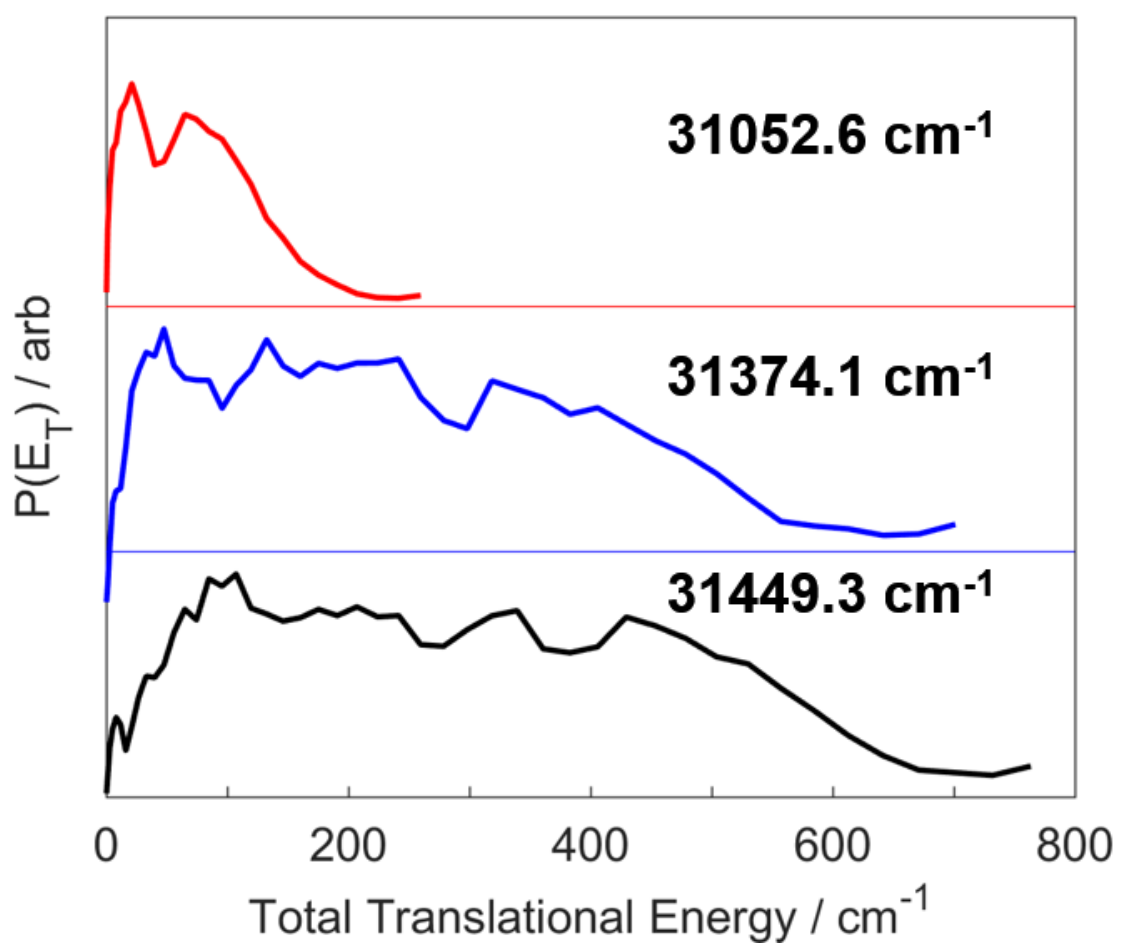

Fig. 5.6 D atom translational energy distributions of three selected images displayed in Figure 5.4.

Excitation of HDCO at all energies resulted in relatively Uniform D atom TEDs, Fig. 5.6.

Those for $\mathrm{H}$ atom peaked at a higher energy corresponding to its nearly $500 \mathrm{~cm}^{-1}$ lower threshold, as is reflected in the $\mathrm{P}(\mathrm{E})$ plots from $31052.6 \mathrm{~cm}^{-1}$ excitation in Fig. 5.6 and 5.7 The lower $\mathrm{H}$ atom threshold also results in more available energy for its cofragment DCO. At the highest excitation energy used, $31449.3 \mathrm{~cm}^{-1}$, the $\mathrm{H}$ atom $\mathrm{P}(\mathrm{E})$ in Fig. 5.7 decreases with increasing translational energy beyond $140 \mathrm{~cm}^{-1}$ then rises again above $\sim 200 \mathrm{~cm}^{-1}$. A total translational energy of $140 \mathrm{~cm}^{-}$ ${ }^{1}$ corresponds to a DCO internal energy of $866 \mathrm{~cm}^{-1}$, closely matching the frequency of the $\sim 850$ $\mathrm{cm}^{-1}$ DCO bending mode. ${ }^{237-242}$ All other vibrational modes are higher in energy and just beyond the available energy. The HCO bending mode, $\sim 1080 \mathrm{~cm}^{-1}$, is not seen in the D atom P(E) in Fig. 5.6 because the excitation energy is insufficient. The $\mathrm{H}$ atom $\mathrm{P}(\mathrm{E})$, particularly at high energy 
beyond the DCO bending mode, does not appear to be purely statistical. Future studies are necessary to investigate the underlying dynamics and potential involvement of the $T_{1}$ barrier. Previously, the distribution of population in HCO states characterized by different values of $K_{c}$, the quantum number describing projection of angular momentum onto the c-axis, was sensitive to initially prepared $K_{c}$ of $\mathrm{H}_{2} \mathrm{CO} .{ }^{233}$ Oscillations in detailed $\mathrm{HCO}$ product state distributions over a more than $300 \mathrm{~cm}^{-1}$ range could not be explained, showing deviations from calculations based on phase space theory when $K_{a}$, the quantum number describing the projection of angular momentum onto the a axis, and $K_{c}$ were included. ${ }^{243}$

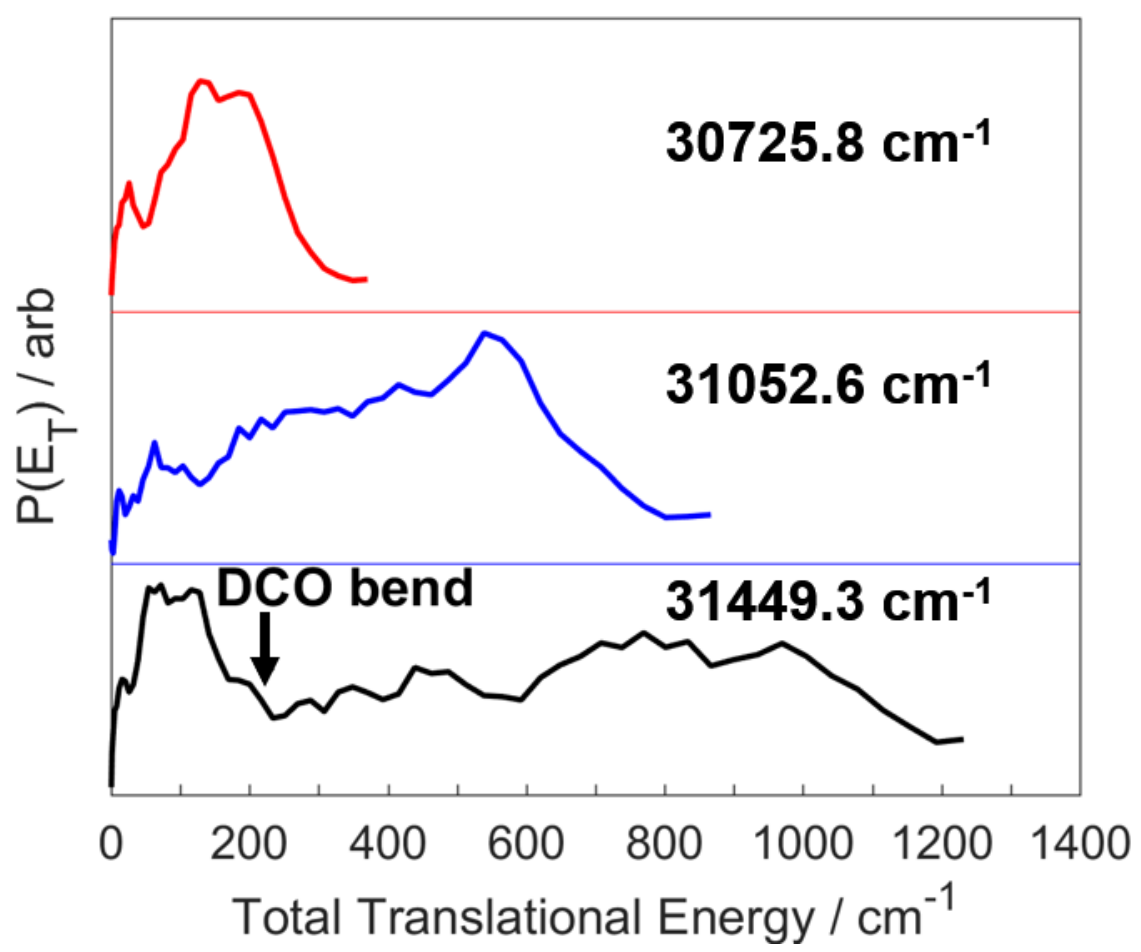

Fig. 5.7 H atom translational energy distributions of three selected images displayed in Figure 5.4.

\subsection{Conclusion}

PHOFEX spectra over a wide range will allow for the assignment of more vibrational bands and determination of spectroscopic constants. Known frequencies of rotational lines can be used to study the dynamics of different vibrational bands and the energy dependence 
of different processes. Accurate ground state radical thresholds obtained from velocity map imaging will assist future HDCO dynamics studies. Threshold phenomena such as competing roaming and non-roaming molecular pathways that occur in $\mathrm{H}_{2} \mathrm{CO}$ require exact threshold energies and an understanding of the underlying dynamics in the radical dissociation channel. The effect on HDCO of excitation at each atomic threshold and the energies in between each may reveal essential information on the roaming mechanism and assist in refining the PESs. 


\section{CHAPTER 6}

\section{QUANTUM ASPECTS OF THE ROAMING PATHWAY IN FORMALDEHYDE PHOTODISSOCIATION}

\subsection{Introduction}

The signatures of formaldehyde roaming dynamics were introduced in Chapter 1. Here I investigate quantum aspects of the mechanism. Quantum modulation and control is of enormous importance as the world inches towards the age of quantum computing. Identification of the influence of quantum mechanics on the dynamics of a system as with as many degrees of freedom as formaldehyde could provide universal insights, similar to the initial roaming study. ${ }^{24,44}$ At the roaming threshold in formaldehyde the $\mathrm{H}$ atom de Broglie wavelength is up to $7 \AA$, behaving like a diffuse wave spreading over the molecule. ${ }^{44}$ Quantization of vibrational and rotational motion gives rise to ZPE, which is a challenge for QCT investigations of roaming. ${ }^{244-246}$ Both roaming and tight TS pathways can produce $\mathrm{CO}$ with moderate rotational excitation, resulting in a range of $j_{\mathrm{CO}}$ states produced from both pathways, as demonstrated in Chapter 1 (Fig. 1.2B). If both $\mathrm{CO}$ and $\mathrm{H}_{2}$ product pairs are produced in the same rovibrational state from both pathways, quantum interferences will occur, but their manifestation is not clear. Quantum mechanics allows for tunneling to radical and molecular products and tunneling or corner-cutting to molecular products via the tight TS or roaming saddlepoint. ${ }^{30,201}$

Formaldehyde exists as two nuclear spin isomers, ortho and para, with a 3:1 statistical weight advantage for the ortho form and no interconversion between them on the timescales of these experiments. The earlier investigations of roaming have focused on ortho formaldehyde owing to the enhanced sensitivity the statistical advantage provides, but some para levels have also been studied and found to give a greater roaming yield on specific CO product states. ${ }^{202}$ Here, a 
detailed examination of the photochemical dynamics in both ortho and para formaldehyde exploring the full range of parent rotational levels from $\mathrm{J}=0$ to 4 is provided. Measurements of the entire $\mathrm{CO}(\mathrm{v}=0)$ product rotational distributions are combined for each with velocity map imaging of selected CO product states to obtain a complete picture of the photochemical dynamics for each specific parent rotational level.

\subsection{Experimental Methods}

$\mathrm{H}_{2} \mathrm{CO}$ monomers were produced by a method adapted from previous experiments in which monomers are liberated from paraformaldehyde upon heating. ${ }^{233}$ Paraformaldehyde, anhydrous $\mathrm{MgSO}_{4}$, and glass wool were packed into a KF elbow before the valve. The KF elbow, valve, and connecting stainless steel parts were wrapped with braided fiberglass heating cord and heated to $\sim 70^{\circ} \mathrm{C}$ to liberate $\mathrm{H}_{2} \mathrm{CO}$ monomers from paraformaldehyde. A 930 Torr helium backing pressure resulted in a $\sim 5 \%$ formaldehyde concentration and a beam temperature of between 10 and $20 \mathrm{~K}$. Beam temperature was approximated from relative populations of $\mathrm{H}_{2} \mathrm{CO}$ rovibrational lines could be adjusted approximately by changing the backing pressure and the valve timing, allowing for an earlier part of the supersonic expansion to be sampled.

UV light was generated by frequency doubled or tripled narrow linewidth $\left(0.1 \mathrm{~cm}^{-1}\right)$ dye lasers pumped by Nd:YAG lasers. Both pump and probe energies were less than $0.2 \mathrm{~mJ} / \mathrm{pulse}$. PHOFEX spectra were obtained by scanning the excitation/dissociation laser from 29460 to 31560 $\mathrm{cm}^{-1}$ for rovibrational bands on $\mathrm{S}_{1}$ and using REMPI detection of various $j_{C O}$ levels via $\mathrm{Q}\left(\mathrm{B}^{1} \Sigma^{+} \leftarrow \mathrm{X}^{1} \Sigma^{+}\right)^{106}$ around $230 \mathrm{~nm}$ and $\mathrm{H}$ atoms via $2+1$ REMPI via $2 \mathrm{~s}\left({ }^{2} \mathrm{~S}_{1 / 2}\right) \leftarrow 1 \mathrm{~s}\left({ }^{2} \mathrm{~S}_{1 / 2}\right)$ near 243

nm..$^{3,110,235,236}$ Vibrational bands on $S_{1}$ covered in this scan range include $2^{1} 4^{1}, 2^{1} 6^{1}, 2^{1} 4^{3}, 2^{2} 4^{1}$, and 
$2^{2} 4^{3}$. Spectral simulations were done using PGOPHER $^{8}$ and a fit was performed to assign rotational structure in vibrational bands of the experimental spectra.

CO REMPI spectra were obtained by fixing the excitation/dissociation laser on a particular parent rovibrational line and scanning the probe laser from 43455 to $43506 \mathrm{~cm}^{-1}$, covering $j_{\mathrm{CO}}=0$ to 63 . Accounting for $2 J+1$ degeneracy weighting provides the $\mathrm{CO}$ rotational distribution from photodissociation at the selected parent state. $\mathrm{H}_{2} \mathrm{CO}$ ground and excited state $J$ were between 0 and 4 , with a rotational energy typically around $6 \mathrm{~cm}^{-1}$ and up to $43 \mathrm{~cm}^{-1}$. Imaging of various $j_{\mathrm{CO}}$ levels was also performed following excitation and dissociation of parent rovibrational lines.

\subsection{Results}

PHOFEX spectra of many formaldehyde vibrational bands of $S_{1}$ have been collected in multiple studies. ${ }^{25,233,247}$ Exact energies of the range of rotational lines, however, have not been reported; more often the value of the transition used is given, usually without specifying which line. A PHOFEX spectrum of the $2^{1} 4^{3}$ vibrational band, was first collected because it is just above the roaming threshold and has a large oscillator strength (Fig. 6.1, top). Rotational lines were assigned by simulating the spectrum (Fig. 6.1, bottom) using PGOPHER. The most intense ortho transitions, such as ${ }^{p} Q_{1,0}(1) \rightarrow 1_{01}$ and the overlapping ${ }^{r} R_{1,0}(1) \rightarrow 2_{21} /{ }^{r} R_{1,1}(1) \rightarrow 2_{20}$ lines, are often used. ${ }^{51,53,204}$ The para transitions, closely spaced asymmetry doublets in the upper state, in the box of Fig. 6.1 were studied here in addition to other ortho transitions. The energy of each $2^{1} 4^{3}$ transition used to excite formaldehyde, lower state energy, and upper state energy can be found in Table 6.1 .

Rotational lines of asymmetric tops are described by the quantum numbers of the upper and lower states in the transition, 


$$
{ }^{\Delta K a} \Delta J_{K a^{\prime \prime}, K c^{\prime \prime}}\left(J^{\prime \prime}\right) \rightarrow J^{\prime} K a^{\prime}, K c^{\prime}
$$

where $J^{\prime \prime}, K_{a}{ }^{\prime \prime}$, and $K_{c}{ }^{\prime \prime}$ are the rotational quantum number in the lower state, the projection of rotational angular momentum onto the $a$ inertial axis in the lower state, and the projection of angular momentum onto the $c$ inertial axis in the lower state, respectively. Analogously, $J^{\prime}, K_{a}{ }^{\prime}$, and $K_{c}{ }^{\prime}$ describe the upper state, and $\Delta J, \Delta K_{a}$, and $\Delta K_{c}$ are the difference between each. Letters $p$, $q$, and $r$ describe $\Delta K_{a}=-1,0$, and 1 , and $P, Q$, and $R$ describe $\Delta J=-1,0$, and 1 , respectively.

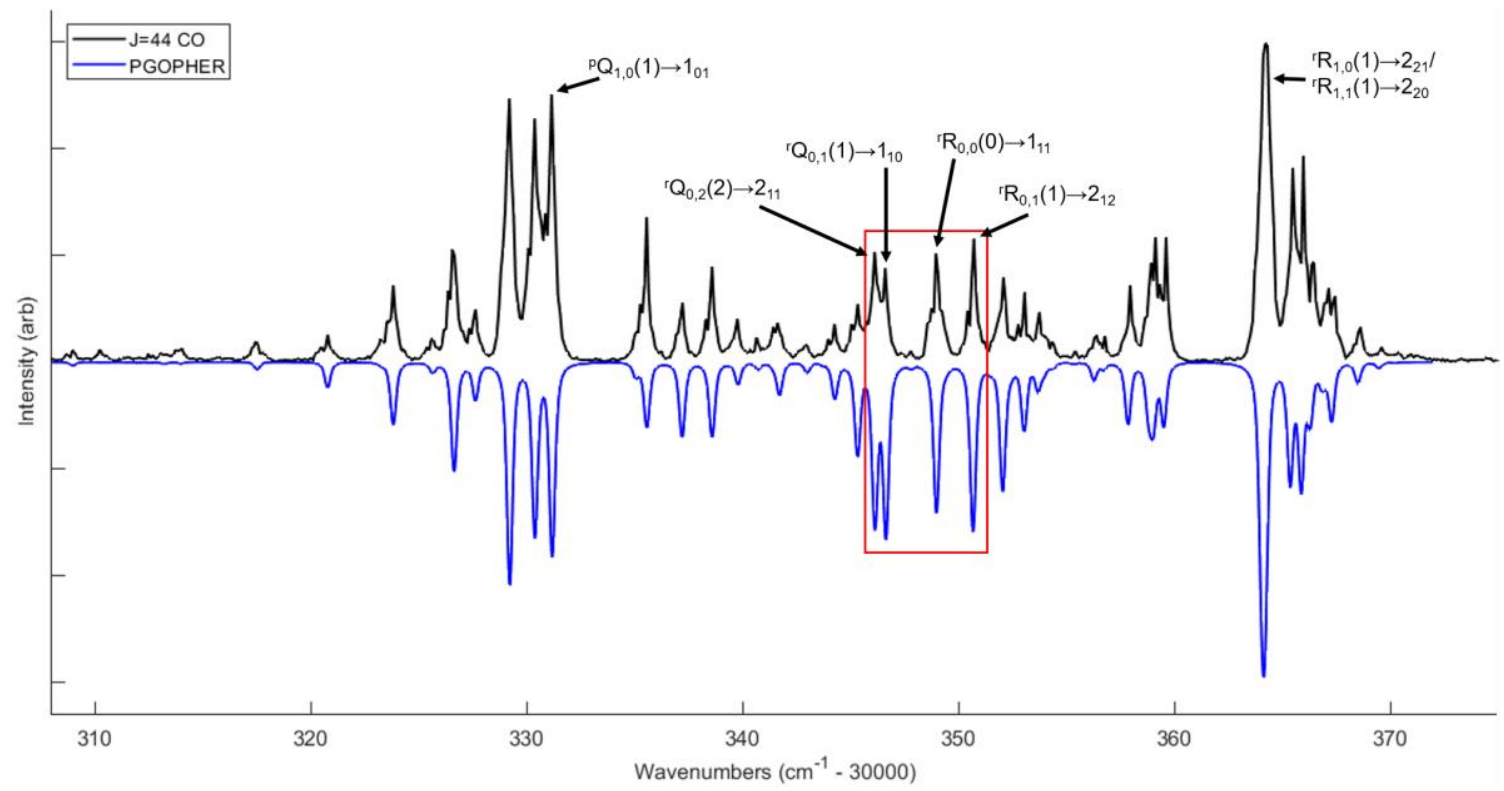

Fig. 6.1 $22^{1} 4^{3}$ PHOFEX spectrum with $j_{\mathrm{CO}}=44$ REMPI detection (top) and PGOPHER simulation (bottom). Commonly used ortho rotational lines and the four para rotational lines are labeled.

CO rotational distributions were collected by fixing the pump laser on each different ortho and para transition and scanning the probe laser. Product $\mathrm{CO}(\mathrm{v}=0)$ rotational distributions, noting that $\mathrm{v}=0$ is the dominant $\mathrm{CO}$ vibrational level produced at this energy, are revealed to be significantly different (Fig. 6.2). Raw spectra not shown in this Chapter are provided in Appendix E. Rotational levels below $j_{\mathrm{CO}}=20$ are largely from roaming events, and those above $j_{\mathrm{CO}}=30$ result almost exclusively from dissociation over the conventional tight TS. Fig. 6.2A shows the distributions following excitation of ortho formaldehyde to the lowest five rotational levels in $S_{1}$, 
$0_{00}$ through $4_{04}$, corresponding to the upper state rotational quantum numbers. Fig. 6.2B gives the analogous plot for the para levels, of which there are four in the same energy region. These are closely spaced asymmetry doublets $1_{10}, 1_{11}$ and $2_{11}, 2_{12}$.

Table 6.1 Rotational lines of $2^{1} 4^{3}$ and their energies

\begin{tabular}{|c|c|c|c|}
\hline Rotational line & Position & Lower state energy & Upper state energy \\
\hline${ }^{\mathrm{p}} \mathrm{P}_{1,5}(5) \rightarrow 4_{04}$ & 30317.5 & 43.4 & 30360.9 \\
\hline${ }^{\mathrm{p}} \mathrm{P}_{1,1}(1) \rightarrow 0_{00}$ & 30329.2 & 10.5 & 30339.8 \\
\hline${ }^{\mathrm{p}} \mathrm{Q}_{1,1}(2) \rightarrow 22_{02}$ & 30330.4 & 15.7 & 30346.1 \\
\hline${ }^{\mathrm{p}} \mathrm{Q}_{1,0}(1) \rightarrow 1_{01}$ & 30331.2 & 10.7 & 30341.9 \\
\hline${ }^{\mathrm{p}} \mathrm{R}_{1,2}(2) \rightarrow 3_{03}$ & 30337.2 & 15.2 & 30352.5 \\
\hline${ }^{\mathrm{r}} \mathrm{Q}_{0,2}(2) \rightarrow 2{ }_{11}$ & 30346.1 & 7.3 & 30353.4 \\
\hline${ }^{\mathrm{r}} \mathrm{Q}_{0,1}(1) \rightarrow 1_{10}$ & 30346.7 & 2.4 & 30349.1 \\
\hline${ }^{\mathrm{r}} \mathrm{R}_{0,0}(0) \rightarrow 1_{11}$ & 30349.0 & 0.0 & 30349.0 \\
\hline${ }^{\mathrm{r}} \mathrm{R}_{0,1}(1) \rightarrow 2_{12}$ & 30350.7 & 2.4 & 30353.1 \\
\hline${ }^{\mathrm{r}} \mathrm{R}_{1,0}(1) \rightarrow 2_{21} /$ & $30364.06 /$ & $10.7 /$ & $30374.76 /$ \\
\hline${ }^{\mathrm{r}} \mathrm{R}_{1,1}(1) \rightarrow 2_{20}$ & 30364.22 & 10.5 & 30374.76 \\
\hline
\end{tabular}

Oscillations in these CO rotational distributions are clearly seen: Those associated with roaming were recently inferred from measurement of the $\mathrm{H}_{2}$ distributions for $\mathrm{v}=6$ and above and discussed further below. ${ }^{248}$ Here we see the entire sweep of the distributions and observe obvious bimodality also in the products from the tight TS with negligible change in excitation energy. At high $j_{\mathrm{co}}$, there appears to be one maximum near $j_{\mathrm{CO}}=32$ and another near $j_{\mathrm{CO}}=45$. These effects 
are not captured by any classical treatment of the dynamics, but it is interesting to note the dip in the main peak can be seen in the distributions reported by van Zee et al. in the early work that anticipated roaming in formaldehyde ${ }^{51}$ as well as in recent measurements from the Kable group. ${ }^{53}$ The para levels also show the same bimodality in the tight TS pathway, with $1_{10}$ resembling 303 , while $1_{11}$ is dominated by the high-jco peak and shows a minimum around $j_{\mathrm{CO}}=32$. We note the difference in excited state energy for these $j_{\mathrm{CO}}=1$ para levels is only $0.09 \mathrm{~cm}^{-1}$ (Table 6.1). The 211 and $2{ }_{12}$ distributions show two minima within the main peak. The roaming regions also show oscillations, but these are generally more subtle. One point is obvious in comparing Fig. 6.2A and 6.2B: except for the $3_{03}$ level, all the ortho distributions are quite similar, but the para distributions vary dramatically. Furthermore, the $3_{03}$ level stands out as distinct from all the others, with the high-jco region resembling $1_{10}$, but also featuring enhanced low jco.

Images of $\mathrm{j}_{\mathrm{CO}}=28,37,45$ obtained by velocity map imaging following excitation of a
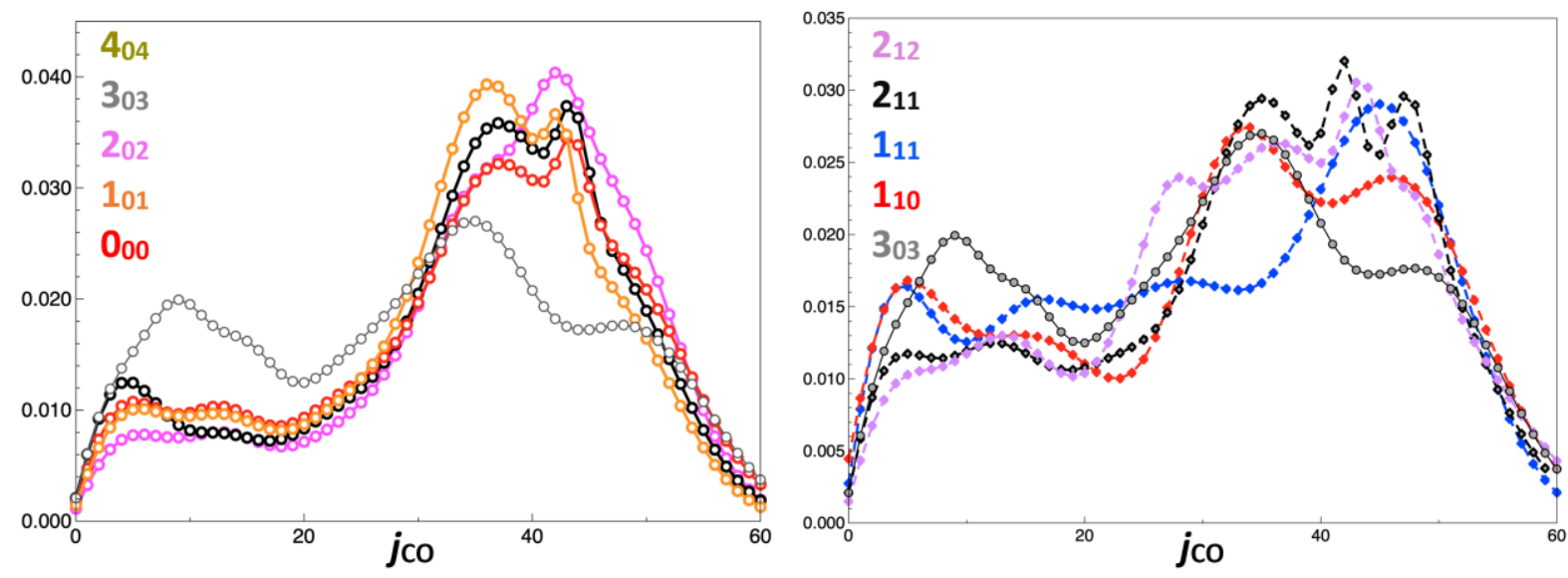

Fig. 6.2 Rotational distributions for $\mathrm{CO}(\mathrm{v}=0)$ following excitation of a range of parent $\mathrm{H}_{2} \mathrm{CO}$ rotational levels on the $2^{1} 4^{3}$ band. All have been normalized to the same total population.

range of parent rotational levels are presented (Figs. 6.3). As discussed in Chapter 1, the low translational energy peaks indicate highly vibrationally excited $\mathrm{H}_{2}$ from roaming, while the high translational energy contribution is from the conventional transition state pathway. 


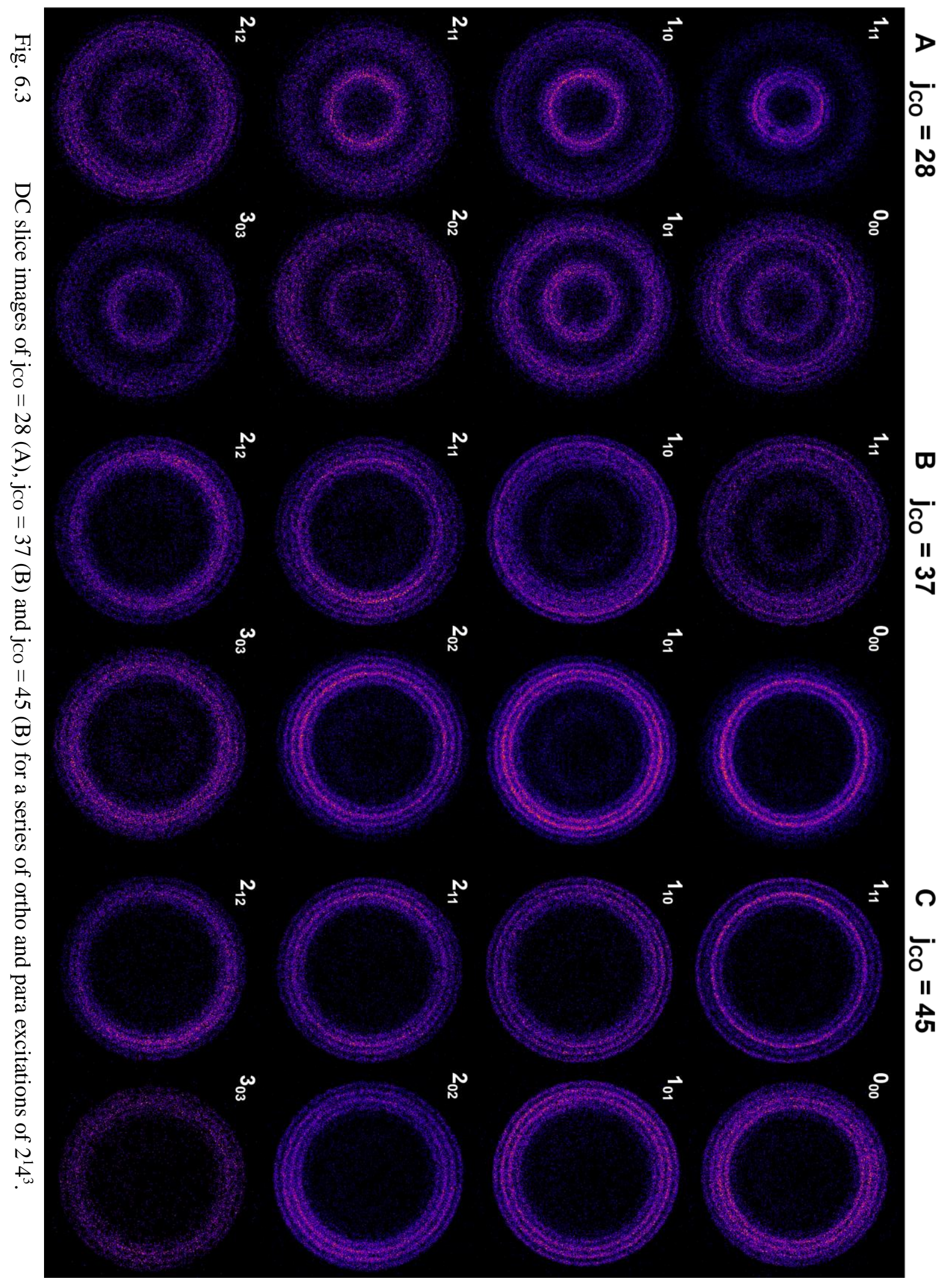


Both para $\left(1_{11}, 1_{10}, 2_{11}\right.$, and $2_{11}$ in the upper state $)$ and ortho $\left(0_{00}, 1_{01}, 2_{02}\right.$, and $3_{03}$ in the upper state) transitions are included. Associated total TEDs, normalized separately with a threepoint smooth applied, in addition to $j_{\mathrm{CO}}=23,32,50$ TEDs are also shown (Figs. 6.4). Low-jco pure roaming distributions are mostly similar in all cases, with minor fluctuations in $v_{\mathrm{H} 2}=7,8$ and 9, and is not shown here. A detailed investigation uncovering bimodality of $\mathrm{H}_{2}(v, j)$ distributions produced via roaming was recently published and perhaps is related to these fluctuations. ${ }^{248} \mathrm{~A}$ more complete set and all associated images and can be found in Appendix C and Appendix D, respectively. The progression from low to high $j_{\mathrm{CO}}$ shows the inner rings corresponding to vibrationally excited $\mathrm{H}_{2}, v_{\mathrm{H} 2}>5$, dissipate for every excitation, as is expected with the long-range roaming reaction incapable of forming high $j_{\mathrm{CO}}$ and correlated low $v_{\mathrm{H} 2}$ (Fig. 6.3). The relative intensity of the inner vs. outer rings wildly vary depending on the excitation. Para transitions clearly exhibit a higher propensity to roam, especially $1_{11}$, than ortho transitions, though $3_{03}$ has relatively more intense inner rings than the other ortho transitions (Fig. 6.3A). Differences in the relative intensities can be seen in $j_{\mathrm{CO}}=37$ (Fig. 6.3B) and $j_{\mathrm{CO}}=45$ (Fig. 6.3C) images, thought they are not immediately as identifiable in the $j_{\mathrm{CO}}=28$ images where roaming can be detected.

Peaks in TEDs reflect the correlated $\mathrm{H}_{2}$ internal state distributions (Fig. 6.4), as indicated by the combs above, and can readily be ascribed to roaming or tight TS pathways as there is little overlap between these at high and low $v_{\mathrm{H} 2}$. Only the $j_{\mathrm{H} 2}=0$ or 1 states are marked in the combs. Nuclear spin is conserved in formaldehyde photodissociation, meaning only even and odd $j_{\mathrm{H} 2}$ sates can be formed for para and ortho excitations, respectively. ${ }^{52,249,250}$ The distributions in Fig. 6.4 have been separately normalized by their areas to facilitate comparison. As observed in the images, the para levels tend to show much greater variation in the correlated state distributions as well as in the roaming fraction in the regions where both roaming and tight TS pathways contribute. The 
ortho levels in Fig. 6.4 show more uniform distributions, and the roaming fraction for each $\mathrm{CO}$ product state is mostly consistent regardless of the parent level excited, although there are some clear deviations, most significantly for $3_{03}$. An interesting exception in the region $j_{\mathrm{CO}}=32-37$, where the ortho levels show significant variance. The shifting TEDs, however, do not follow any trend with $\mathrm{J}$ of the ortho transition. This comparison is quantified by the variance, $\mathrm{s}^{2}$, indicated for each $j_{\mathrm{CO}}$ product level in each figure. We see an interesting exception in the region $\mathrm{j}_{\mathrm{CO}}=32-39$.

In that region, the ortho levels show much greater variance while for the para levels it is reduced
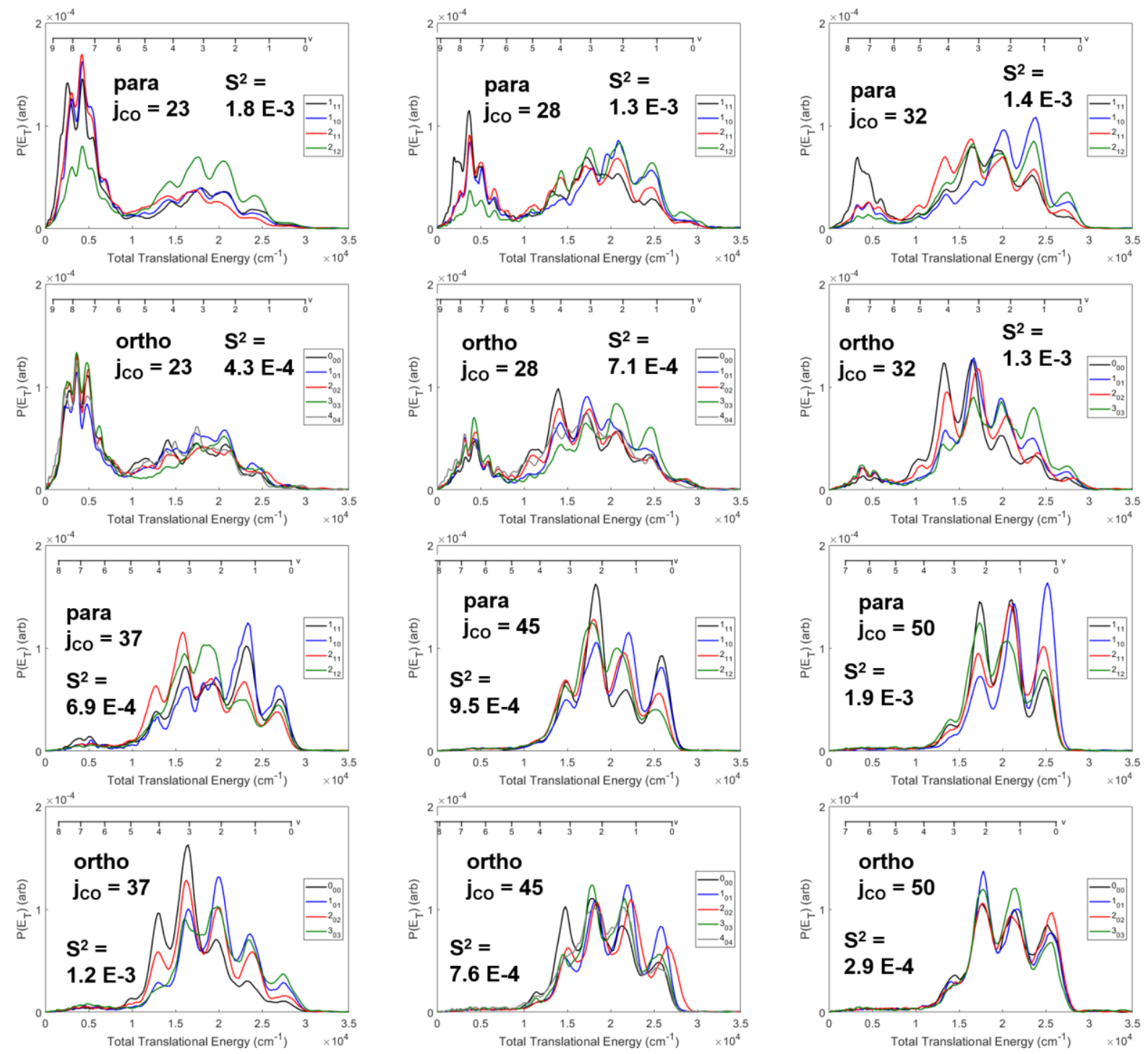

Fig. 6.4

Total translational energy distributions of a series jco images for a series of ortho and para excitations of $2^{1} 4^{3}$. 
there. For purely tight TS pathways, $j_{\mathrm{CO}}=50$ TEDs are notably similar, though the $j_{\mathrm{CO}}=45 \mathrm{TED}$ for $0_{00}$ has a larger population of $v_{\mathrm{H} 2}=3$. The TEDs from para transitions exhibit extreme differences where roaming is a possible pathway, and similar observations apply for the $j_{\mathrm{CO}}=50$ TED, where the relative $v_{\mathrm{H} 2}=0-2$ populations fluctuate for every transition. The sum of these observations indicates that the underlying dynamics are not governed by one phenomenon. Excitation via different rotational lines not only modulate the branching where roaming and tight TS pathways are present, but also affect TEDs associated exclusively from the tight TS pathway. Furthermore, the varying dynamics are not exclusive to para transitions, but can also be seen for ortho transitions, and the drastically different TEDs are affected by altering the upper state energy by only $2 \mathrm{~cm}^{-1}$.

The roaming threshold is first crossed on vibrational band $2^{1} 6^{1}$, which has an origin value of $\mathrm{T}_{0}=30252 \mathrm{~cm}^{-1}$, close to that of $2^{1} 4^{3}, \mathrm{~T}_{0}=30340 \mathrm{~cm}^{-1}$. Though not analyzed as thoroughly as $2^{1} 4^{3}$ here, similar effects are observed (Fig. 6.5). The $j_{\mathrm{CO}}=23$ images (Fig. 6.5A) and associated TEDs (Fig. 6.5C) show modulation of the roaming population, with more produced from $1_{11}$ compared to $1_{10}$. An effect is not observed for $j_{\mathrm{CO}}=45$ images (Fig. 6.5B) and associated TEDs (Fig. 6.5D). The correlated $v_{\mathrm{H} 2}=0-2$ population of $j_{\mathrm{CO}}=45$ varies for in a similar way to $2^{1} 4^{3}$. Images and TEDs are noisier due to weaker transition strength of rotational lines on a vibrational band involving $v_{6}$ instead of $v_{4}$.

Below the roaming threshold, the tight TS pathway governs the formation of $\mathrm{j}_{\mathrm{co}}$ and rotational distributions are unaffected by the roaming pathway. The closest vibrational band to threshold is $2^{1} 4^{1}$. Excitation via a range of ortho and para levels produce $\mathrm{CO}$ rotational distributions with extremely different structures that can be seen in the raw REMPI spectra (Fig. 6.6). The varying structure of the rotational distributions confirm the presence of complicated dynamics 
related with only the tight TS pathway similar to those observed in high $\mathrm{j}_{\mathrm{Co}}$ images above the roaming threshold.

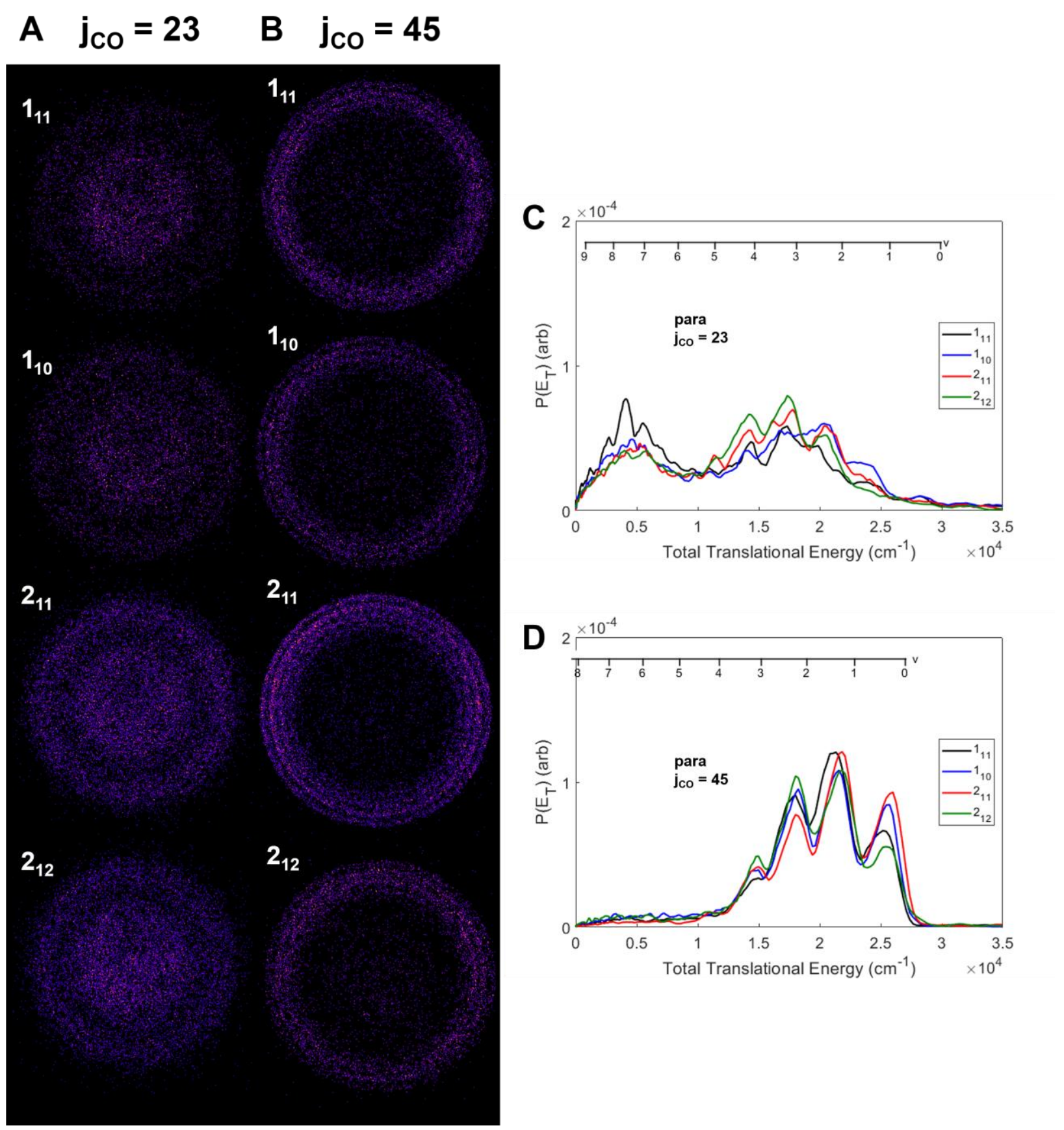

Fig. 6.5 DC slice images of a series of $2^{1} 6^{1}$ para excitations of $j_{\mathrm{CO}}=23(\mathrm{~A})$ and $\mathrm{j}_{\mathrm{CO}}=45(\mathrm{~B})$. Associated total translational energy distributions of $\mathrm{j}_{\mathrm{CO}}=23(\mathrm{C})$ and $\mathrm{j}_{\mathrm{CO}}=45$ (D).

The observations presented thus far have been near the radical and roaming thresholds, where alternate dynamical effects may dominate that are not present or are obscured at higher 
energies. Higher energy vibrational bands $2^{2} 4^{1}, \mathrm{~T}_{0}=30659 \mathrm{~cm}^{-1}$, and $2^{2} 4^{3}, \mathrm{~T}_{0}=31532 \mathrm{~cm}^{-1},{ }^{197}$ were thus investigated in a similar manner as $2^{1} 4^{3}$. Initial investigations were focused on para transitions, and there is no ortho data for these vibrational bands. PHOFEX spectra for $2^{2} 4^{1}$ and $2^{2} 4^{3}$ were collected first using different $j_{\mathrm{CO}}$ level and $\mathrm{H}$ atom REMPI detections methods. Because these vibrational bands contain the same modes, $v_{2}$ and $v_{4}$, with different quanta, PHOFEX spectra generally have the same structure and relative intensities. Different spectra will only be presented when they aid discussion, and the rest can be found in Appendix E. Presently, we examine $j_{\mathrm{CO}}=$ 23 and 45 images for $2^{2} 4^{1}$ and $2^{2} 4^{3}$. Images for $2^{2} 4^{1}$ (Fig. 6.7A and 6.7B) and $2^{2} 4^{3}(6.7 \mathrm{C}$ and D) do not have immediately discernible differences. The associated TEDs for the $j_{\mathrm{CO}}=23$ and 45 images (Fig. 6.8) confirm that the parent state does not significantly affect the TEDs as the para transitions of $2^{1} 4^{3}$ did, except for $v_{\mathrm{H} 2}=0-3$ population of $j_{\mathrm{CO}}=45$ varying for $2^{2} 4^{1}$. The roaming populations in $j_{\mathrm{CO}}=23$ do have minor fluctuations for $2^{2} 4^{1}$, but TEDs for $2^{2} 4^{3}$ are unaffected.

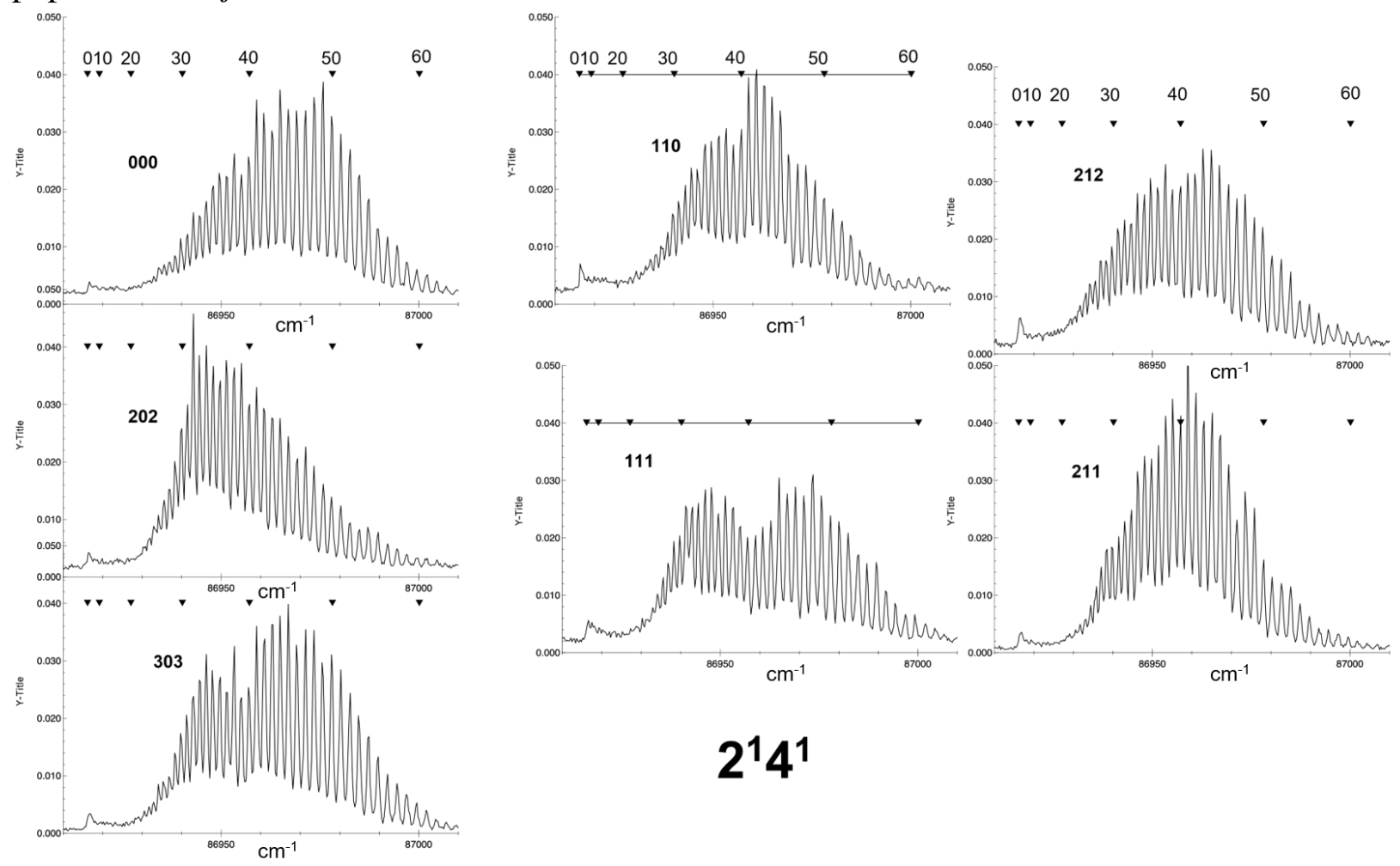

Fig. 6.6 Raw CO REMPI spectra for several ortho and para excitations on the $2^{1} 4^{1}$ band. 
The overall roaming fraction for a particular $\mathrm{H}_{2} \mathrm{CO}$ parent state can be determined by calculating the roaming fraction for each TED (defined as the fraction giving $\mathrm{H}_{2} v>5$ ), then weighting these by the rotational distributions in Fig. 6.2. This is shown for two examples in Fig.

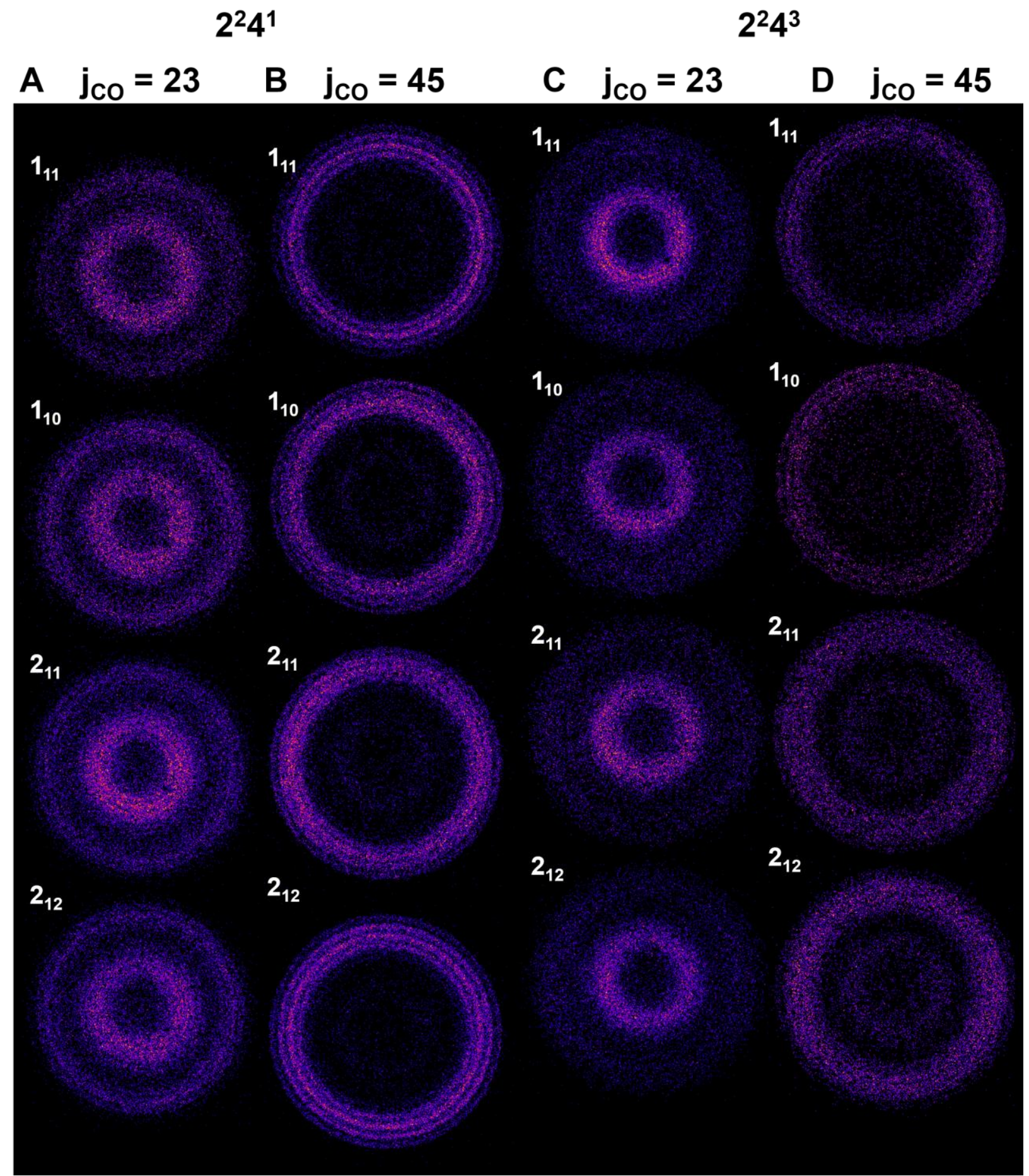

Fig. 6.7 DC slice images of $j_{\mathrm{CO}}=23$ dissociated from $2^{2} 4^{1}(\mathrm{~A}), j_{\mathrm{CO}}=45$ dissociated from $2^{2} 4^{1}$ $(\mathrm{B}), j_{\mathrm{CO}}=23$ dissociated from $2^{2} 4^{3}(\mathrm{C}), j_{\mathrm{CO}}=45$ dissociated from $2^{2} 4^{3}(\mathrm{D})$. 
6.9. Integration of this weighted distribution for all products gives us the overall roaming fraction, also shown in Fig 6.9. The roaming fraction for the para levels is $\sim 30 \%$, while for the ortho levels it is $\sim 20 \%$ apart from the anomalous 303 level. That level yields the highest roaming fraction, $35 \%$. The total roaming fraction varies by a factor of 2 between the 202 and 303 levels, a difference in excitation energy of only $4 \mathrm{~cm}^{-1}$. It is the large variation in the rotational distributions rather than the variation in a given TED that chiefly determines the overall roaming fraction for a given excited level. Results for the ortho levels are largely consistent with previous measurements of Lahankar et al. $(\sim 18 \%)^{52}$ and Kable and coworkers $(\sim 16 \%)^{53}$ but in both cases this was obtained for a single transition (to $22_{21} / 2_{20}$ ); the surprising variations seen here suggest that a single level measurement cannot give the full picture, and this is true for roaming as well as the tight TS pathway.
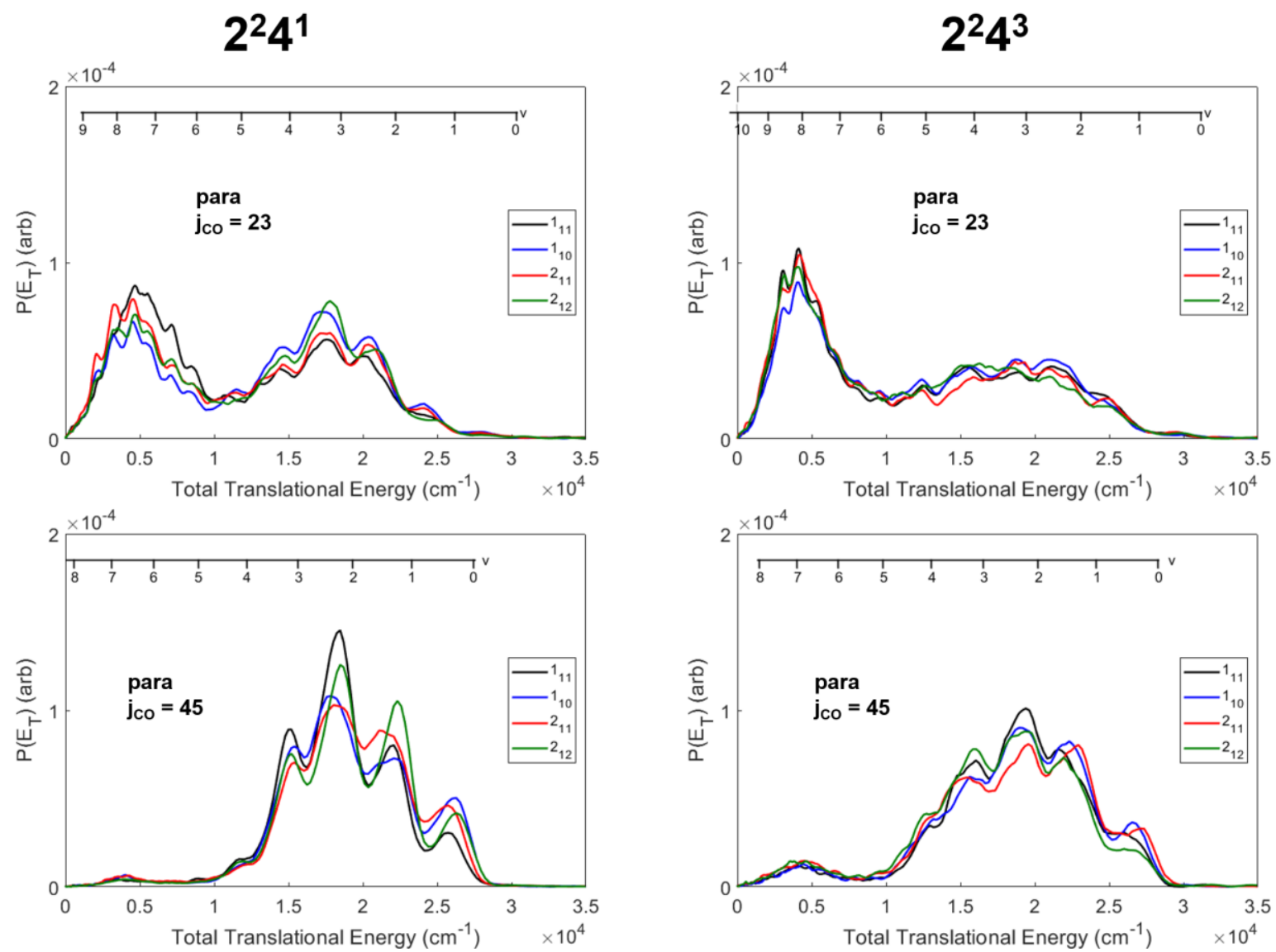

Fig. 6.8 Associated total translational energy distributions for the images in Fig. 6.7 for a series of ortho and para excitations of $2^{2} 4^{3}$ (left) and $2^{2} 4^{3}$ (right). 

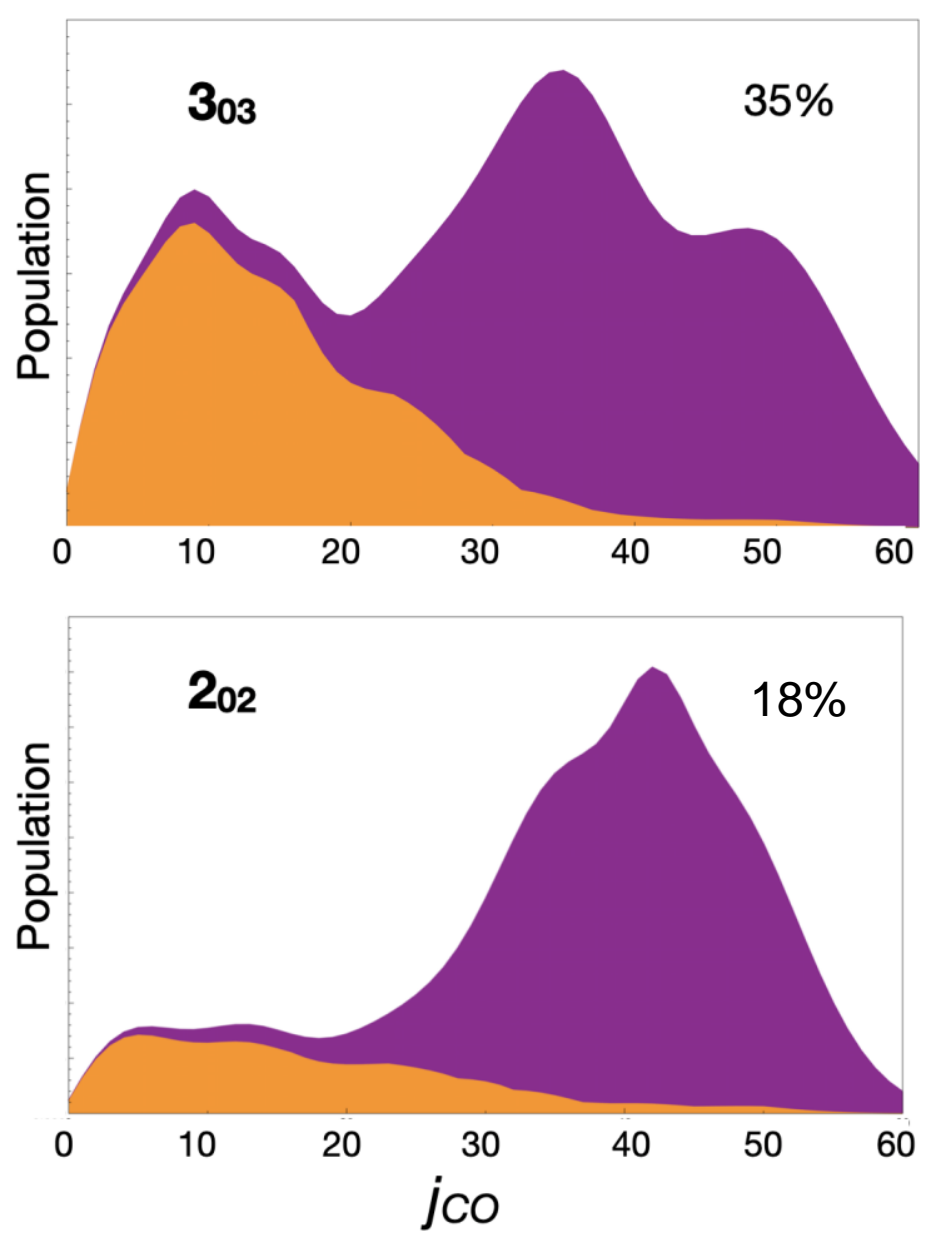

Fig. 6.9 Roaming fraction (orange) relative to the overall CO rotational distribution (purple) for $3_{03}$ (top) and $2_{02}$ (bottom) on $2^{1} 4^{3}$.

\subsection{Discussion}

Now we seek a mechanism that could give rise to these huge variations in the product state distributions associated with changes in parent quantum state but only small variations in total energy. We should state at the outset that our understanding of these complex dynamics is still developing. We start by making clear that these are quantum effects. All the full-dimensional theoretical treatments of roaming in $\mathrm{H}_{2} \mathrm{CO}$ to-date have employed QCT methods, ${ }^{23,27,54,196,202,251}$ and these were key in identifying the roaming mechanism in the first place. ${ }^{24}$ They have largely been successful at capturing the broad features of the dynamics. One such study examined the effect of parent rotation on formaldehyde decomposition both for the roaming and radical 
channels. They found moderate suppression of the roaming fraction at threshold (from $18 \%$ for total $J=0$ to $16 \%$ for $J=30$ ) and this became a modest enhancement at higher excitation energies. ${ }^{252}$ However, nothing resembling the dramatic effects we see here were reported, nor have our collaborators Guo and Xie seen them in QCT investigations of the effect of parent rotation. They have also used QCT to explore the effect of the initial geometry on $\mathrm{S}_{0}$ accessed by internal conversion, comparing bend excited to planar geometries. No differences were seen, and this is also consistent with previous reports. ${ }^{170,251,253}$

As classical treatments are unable to capture the effects we observe, we turn to considering the quantum properties of the dynamics. For this system at the energies considered here, a full dimensional quantum treatment is currently out of reach, so we will seek a qualitative picture to account for these observations. There are several possibilities, and it is likely that all play a role to some extent. These involve processes that depend on the symmetry of the initially prepared rotational level, Coriolis coupling following internal conversion, nearby coincidental resonances accessed following internal conversion, and differential loss to the radical channel that varies with rotational state.

To this end we first consider the symmetry of the initially prepared state in $S_{1}$, its coupling to $\mathrm{S}_{0}$, and how this can impact the subsequent dynamics in $\mathrm{S}_{0}$. Ergodicity has long been used to explain the dynamics following internal conversion to $S_{0}$ and estimated from Stark tuning spectra near the energy of the $\mathrm{S}_{0}$ saddle point. ${ }^{194,254}$ Moreover, in QCT studies, no effects associated with initial geometry have been seen, supporting the notion that, at these energies, the system is largely ergodic. Results here show clearly this is not the case: strict ergodicity is inconsistent with observations such as that odd para transitions lead to a larger roaming population or the consistent differences between $v_{\mathrm{H} 2}$ tight TS pathway from odd and even para transitions. When studying the 
effect of $2^{1} 4^{1}$ parent rotational state on fragment anisotropy, Butenhoff and coworkers observed what appeared to be random variations in anisotropy, with $\beta$ varying from 0 to 0.5 for a range of $J$ $=0$ to 4 para transitions. ${ }^{203}$ They also observed fluctuations in maximum jco and fluorescence lifetimes when different parent rotations were prepared. ${ }^{204}$ The results were attributed to the chaotic nature of the vibrational wavefunction projecting on the TS, with the configuration of the molecule at the moment of dissociation directly affecting the vibrational excitation of the $\mathrm{H}_{2}$ fragment and the rotational distribution of the $\mathrm{CO}$ fragment. These experiments were below the roaming threshold, though some experiments performed above threshold have observed para transitions enhance roaming, with QCT was not able to match the experimental result. ${ }^{202}$

Now we consider the effects of Coriolis interactions in the dynamics on $\mathrm{S}_{0}$. The relevant symmetry considerations are summarized in the diagram in Fig. 6.10, which is framed in terms of the symmetry of the zero-order normal mode vibrations. These are mixed by Coriolis interactions, ${ }^{255}$ i.e., vibration-rotation coupling, an effect that plays a significant role in intramolecular vibrational energy redistribution. ${ }^{256,257}$ This coupling preserves the overall rovibrational symmetry but destroys the "goodness" of the $K_{a}$ and $K_{c}$ quantum numbers and changes the vibrational character of the wavefunction. Absorption to the non-planar $\mathrm{S}_{1}$ state from the vibrational ground state of $\mathrm{H}_{2} \mathrm{CO}$ is nominally forbidden but vibronically allowed for odd values of out-of-plane bend $\left(v_{4}, b_{1}\right.$ symmetry) or asymmetric in-plane bends $\left(v_{5}\right.$ and $v_{6}, b_{2}$ symmetry). $\mathrm{S}_{1}$ has $a_{2}$ electronic symmetry, while the ground state and the molecular products are $a_{1}$. Internal conversion back to $S_{0}$ thus involves a change in electronic character from $a_{2}$ to $a_{1}$, and this is compensated by odd changes in $b_{1}$ and $b_{2}$ symmetry vibrations to preserve the total vibronic symmetry. The rotational states established in the photoexcitation are not changed in the internal conversion, so we have labeled these and discuss them according to the states as they are prepared 
in $\mathrm{S}_{1}$. The "good quantum numbers," those that are conserved regardless of the decay pathway, include total $J$, its space-fixed projection, $M_{J}$, and total parity.

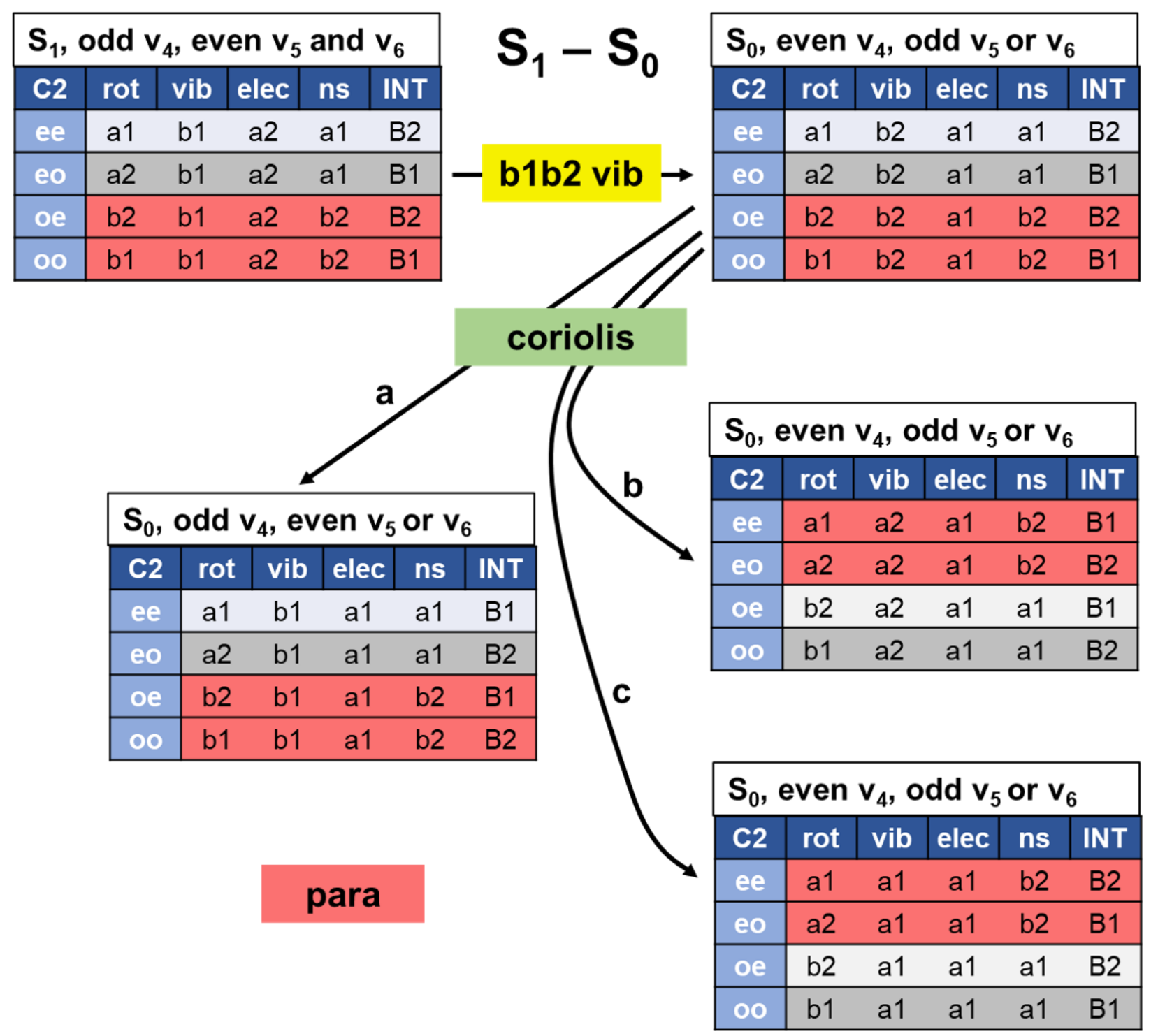

Fig. 6.10 Symmetry coupling "blocks" describing how an initially prepared state on $\mathrm{S}_{1}$ couples to $\mathrm{S}_{0}$.

There are four rotational symmetry species that vary as the values of $K_{\mathrm{a}}$ and $K_{\mathrm{c}}$ are even or odd, and four vibrational symmetries possible reflecting the four symmetry species of the $G_{4}$ molecular symmetry group of the molecule, which is isomorphic to $\mathrm{C}_{2 \mathrm{v}}$ (Fig. 6.10). The total internal symmetry of formaldehyde is either $B_{1}$ or $B_{2}$, odd or even total parity, respectively. These together then give four zero-order symmetry "blocks" as shown in Fig. 6.10, of odd or even total 
parity and ortho or para nuclear spin, but with different vibrational symmetry. Although the total rovibrational symmetry and parity will be maintained on $\mathrm{S}_{0}$, Coriolis coupling will mix these zeroorder levels. These effects in formaldehyde have been studied in detail by Field and coworkers at lower excitation energies and including higher values of $J$ and $K_{a}$ than considered here, ${ }^{255}$ but their discussions are relevant for the present observations. The internal conversion populates an initial state of $b_{2}$ vibrational symmetry (even $v_{4}$, odd, $v_{5}$ or $v_{6}$ ); a-axis Coriolis coupling can give $b_{1}$ vibrational symmetry, b-axis gives $a_{2}$ vibrational symmetry (odd $v_{4}$ and $v_{5}$ or $v_{6}$ ), and c-axis gives $a_{1}$ vibrational symmetry. At these high vibrational energies, the density of states is very high and Coriolis coupling among higher rotational levels is effectively complete. However, for lower rotational levels selection rules can limit or eliminate such coupling. For $J=0$, clearly there is no Coriolis interaction. The approximate selection rules are that $a$-axis coupling is proportional to $K_{\mathrm{a}}$, and $b$ - or $c$-axis coupling is proportional to $J-K_{\text {a. }}$.

For $K_{a}=0$, i.e., all the ortho levels considered here, there is thus no $a$-axis coupling. This means there is no coupling to zero-order vibrational levels that are odd $b_{1}$ (out-of-plane) character. However, $b$-axis coupling is permitted for these levels and will populate levels of $a_{2}\left(b_{1}+b_{2}\right)$ symmetry. One clear difference between these lowest ortho levels and the para levels is thus the absence of $a$-axis Coriolis coupling for the former, and the more limited or vanishing $b$ - and $c$-axis coupling for the respective odd and even total parities of the latter. For the para levels, odd $b_{1}$ vibrational character indicates a node in the plane and thus less pure planarity, suggesting reduced tight TS dissociation events and enhanced roaming, as the tight TS is planar and roaming is known to be favored by non-planar trajectories..$^{30,52,195}$ The ortho levels have complementary behavior. Although this qualitatively accounts for the effects we observe for the para levels, it is not 


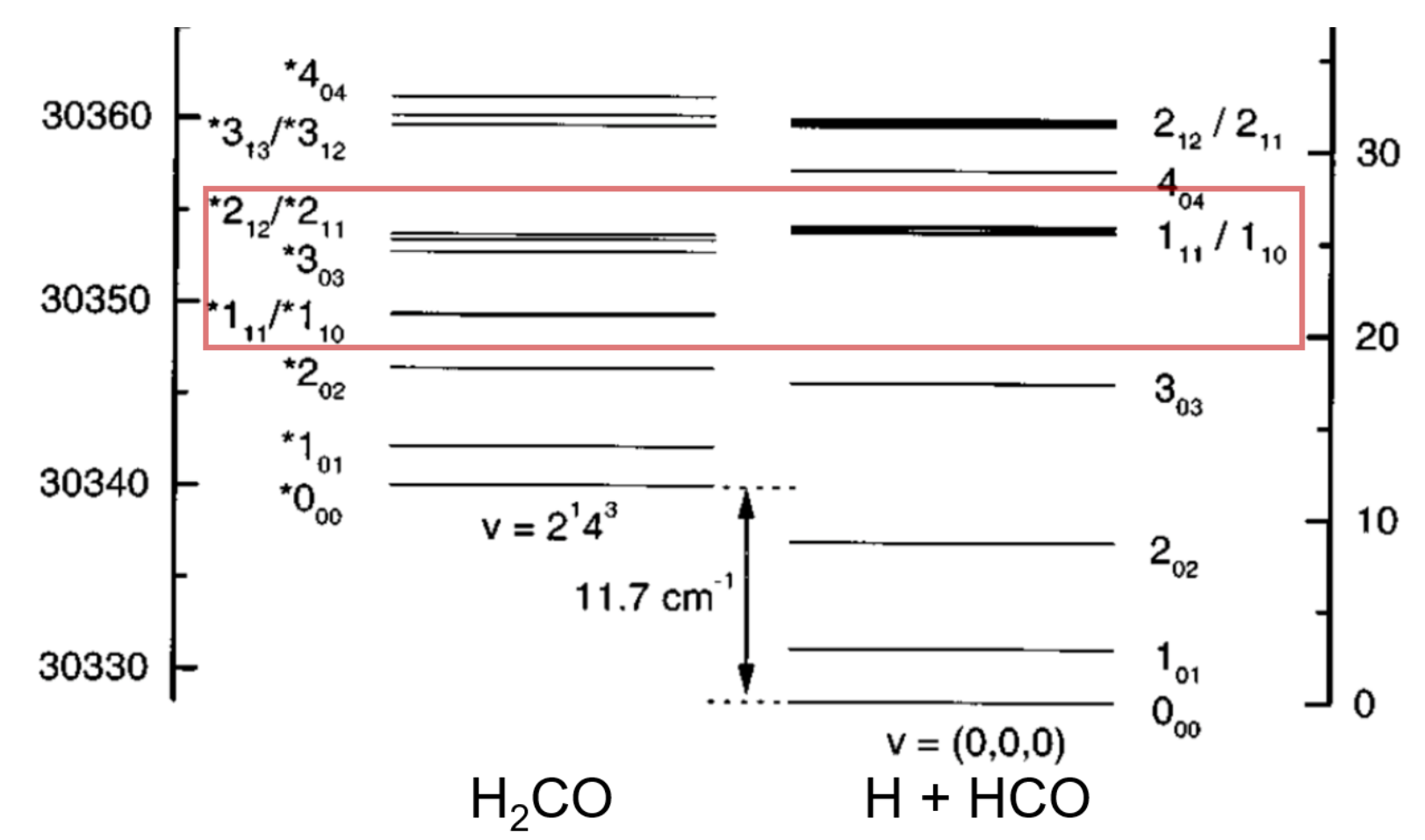

Fig. 6.11 Energy ladder of $\mathrm{H}_{2} \mathrm{CO}$ (left) and $\mathrm{H}+\mathrm{HCO}$ (right) rotational states. The states involved in a resonance are indicated by the red box. Adapted with permission from A.C. Terentis et al, J. Chem. Phys., 108 (8), 3187-3198 (1998) (C) AIP.

consistent with the very large roaming yield on 303 , and the fact that the other commonly studied $K_{a}=2$ ortho levels show little roaming resembling the other ortho levels aside from $3_{03}$.

The dynamics following excitation to $3_{03}$ can be explained by resonant coupling of the parent $\mathrm{H}_{2} \mathrm{CO}$ state to $\mathrm{H}+\mathrm{HCO}\left(1_{11} / 1_{10}\right)$. The $3_{03}$ level (Fig. 6.11 , left) is within $5 \mathrm{~cm}^{-1}$ of $\mathrm{H}+\mathrm{HCO}$, $1_{11} / 1_{10}$ (Fig. 6.11, right), while all other ortho levels are not within $10 \mathrm{~cm}^{-1}$ of such a resonant coupling. ${ }^{233}$ These levels can be brought into resonance in the roaming region, permitting $a$-axis Coriolis interaction through a decoupling of the body-fixed and space-fixed frames, which promotes out-of-plane motion, and is favored by roaming trajectories. $\mathrm{H}+\mathrm{HCO}\left(1_{11} / 1_{10}\right)$ can be resonant with the $\mathrm{H}_{2} \mathrm{CO}$ states in the red box of Fig. 6.11 when $\mathrm{H}$ is in the 3 to 5 angstrom region, ${ }^{233}$ so it greatly impacts the roaming yield and at the same time strongly modulates the tight TS channel. The nature of this resonance is still being understood, but it clearly has a strong effect on 
three pathways, exhibited by differences in the rotational distributions. Roaming is effectively a cold collision of a hot molecule, and the dynamics here resemble that of orbiting collisions. Orbiting resonances have been manifested in different ways, ${ }^{258}$ one study saw broadening of rotational linewidths from van der Waals predissociation. ${ }^{259}$ Roaming in formaldehyde is more complicated, where it is an $\mathrm{H}$ atom scattering off an asymmetric top. In Fig. 6.12 we plot the roaming fraction against the energy of each level relative to the $2^{1} 4^{3}$ origin. Evidence of a resonance can clearly be seen near the para transitions, marked by blue diamonds, and the single $3_{03}$ ortho transition in this window. The region of this resonance is indicated in the blue box of Fig. 6.12, and the oscillating structure of the accompanying $\mathrm{CO}$ rotational distributions from these excitations are also included. It may well be that that $\mathrm{H}+\mathrm{HCO}\left(1_{11} / 1_{10}\right)$ leads to greatly enhanced
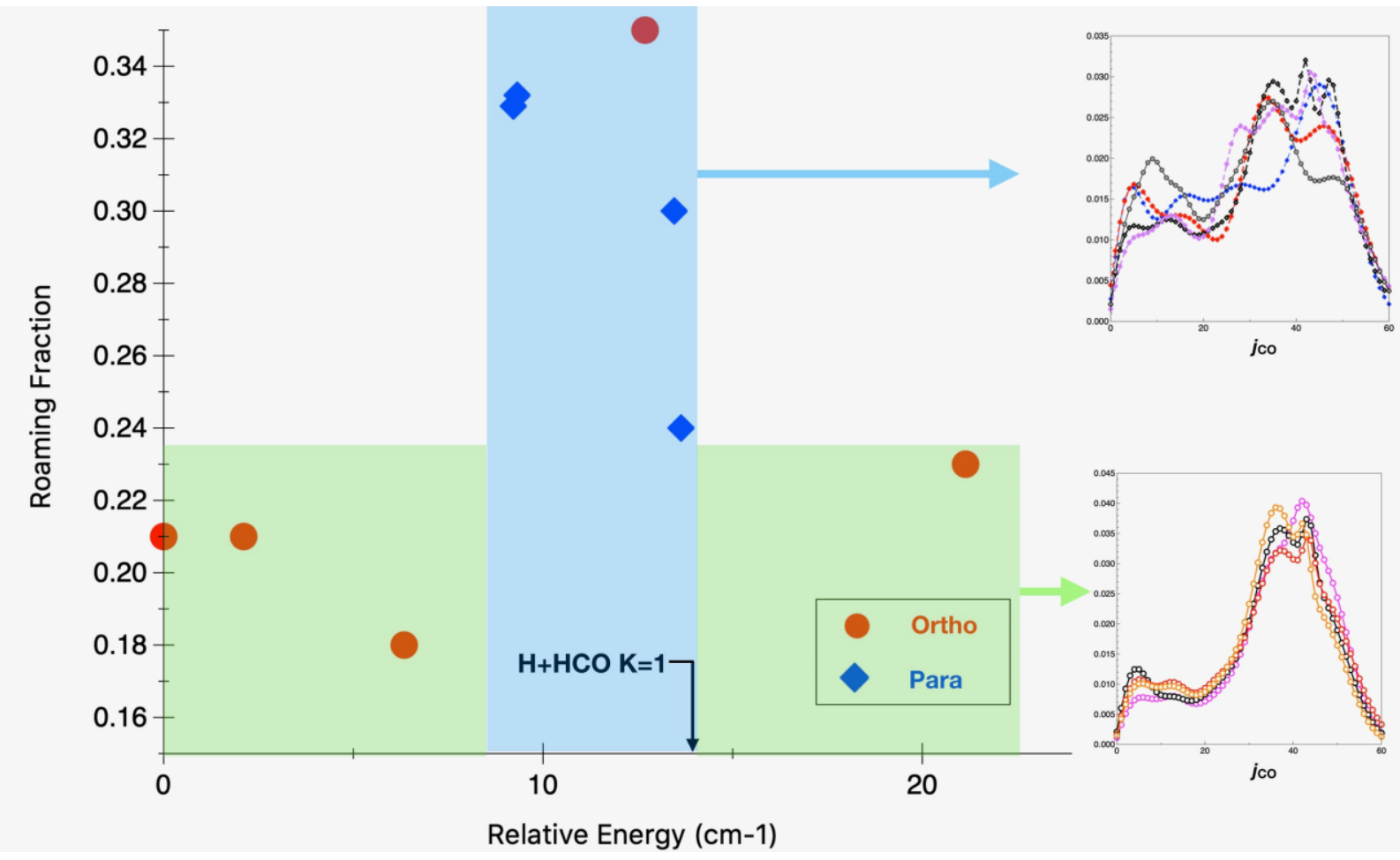

Fig. 6.12 Relative energy vs roaming fractions for ortho (red circles) and para transitions (blue diamonds). The region where a resonance between $\mathrm{H}_{2} \mathrm{CO}$ and $\mathrm{H}+\mathrm{HCO}$ states is observed is indicated by the blue box, with associated $\mathrm{CO}$ rotational distributions. Other ortho transitions (green box) and associated $\mathrm{CO}$ rotational distributions are also included. 
a-axis Coriolis coupling into $b_{1}$ symmetry, orbiting of the $\mathrm{H}$ atom around the $a$-axis and enhanced roaming. Furthermore, whether the out-of-plane motion travels in two directions around HCO and how it affects the roaming yield must be determined. While we present Coriolis coupling as a possible contributing factor to parent rotational state-dependent dynamics, the orbiting resonance clearly dominates any underlying dynamics or resonances. Considering the translational energy
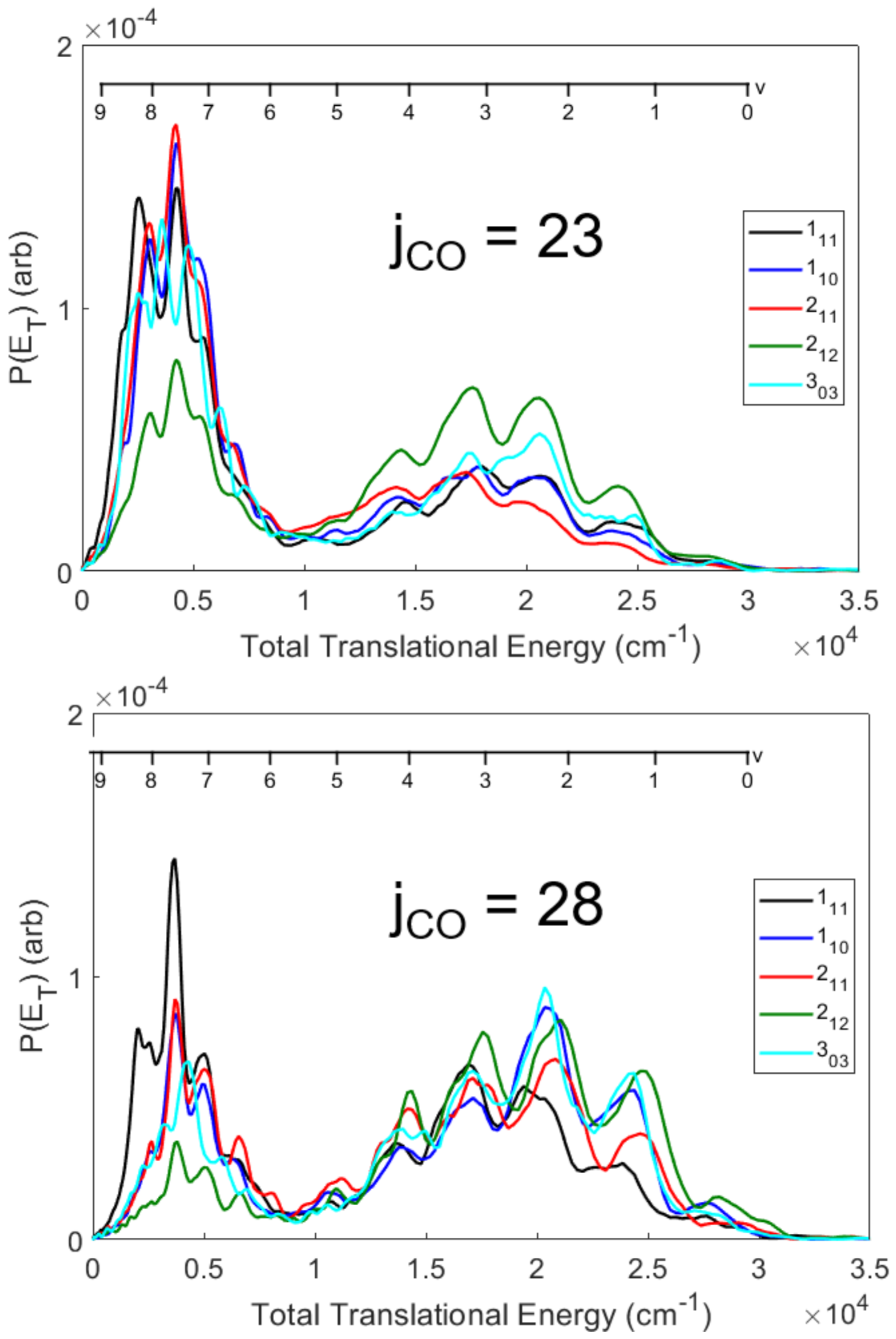

Fig. 6.13 Total translational energy distributions for $\mathrm{j}_{\mathrm{CO}}=23$ and 28 for excitations near the identified resonance. 
distributions again, we note $3_{03}$ shows similar structure to the $\mathrm{J}=1$ para transitions (Fig. 6.13), and the resonance is likely strongest in between energies of these states.

To explore possible distinct dynamics associated with planar vs. non-planar pathways, we compared in-plane restricted and normal dissociation events via QCT and the results are given in Fig. 6.14. In-plane restricted trajectories showed a decrease in the roaming population, indicating that if formaldehyde were dynamically restricted to an in-plane geometry, the roaming population would be reduced, and more high jco levels would be populated. Conversely, if in-plane trajectories were excluded, roaming would be enhanced and the tight TS pathway would be reduced, supporting that the pathways are interconnected. Though Harding and coworkers believed that the pathways are dynamically distinct, another barrierless pathway between the roaming saddle point and tight TS has been suggested. ${ }^{260}$ We performed a 3D quantum mechanical model using the coordinates $\mathrm{R}, \theta$, and $\varphi$, where $\mathrm{R}$ is the distance between the roaming $\mathrm{H}$ atom and the HCO center of mass, $\theta$ is the dihedral angle, and $\varphi$ is the out-of-plane angle. HCO was fixed at its equilibrium geometry, and the energy was at $33082 \mathrm{~cm}^{-1}$, which is $5 \mathrm{~cm}^{-1}$ below the $\mathrm{H}+\mathrm{HCO}$ asymptote on the PES. With $\theta=78^{\circ}$, the wavefunction extends out-of-plane across many configurations, including regions where roaming saddle point and tight TS structures can be accessed (Fig. 6.15).
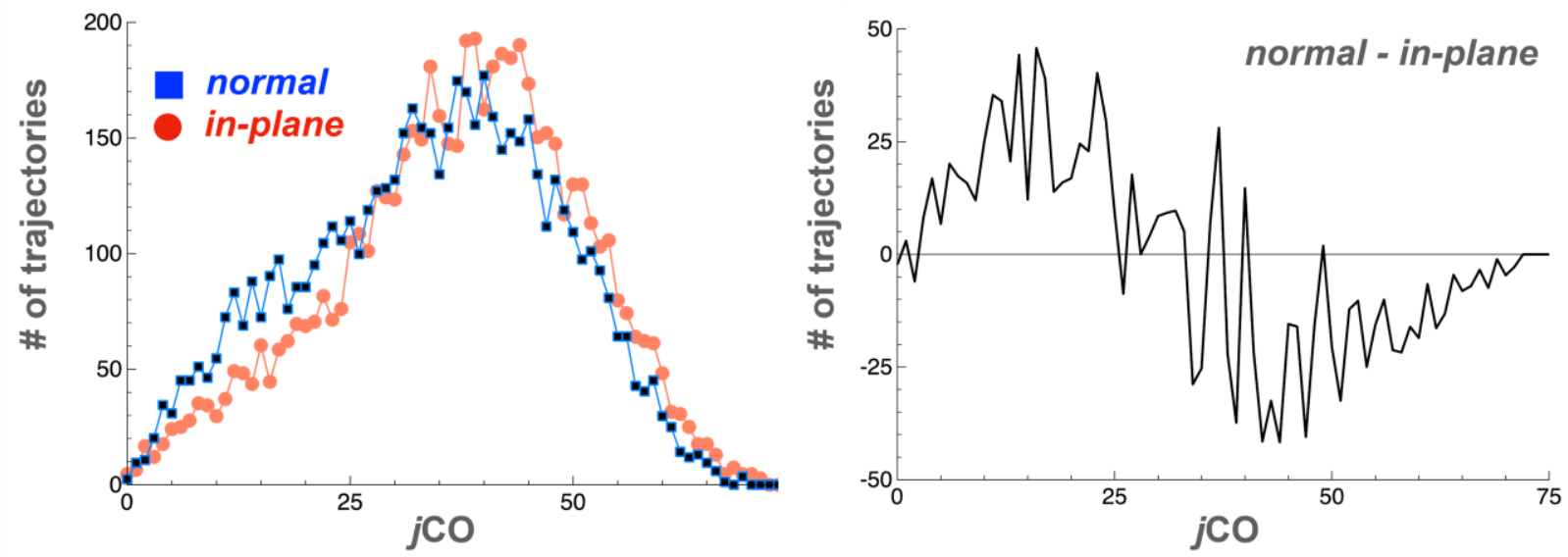

Fig. 6.14 In-plane restricted (red) vs. normal (blue) trajectory results. 


\section{$\theta=78.36 \mathrm{deg}$}

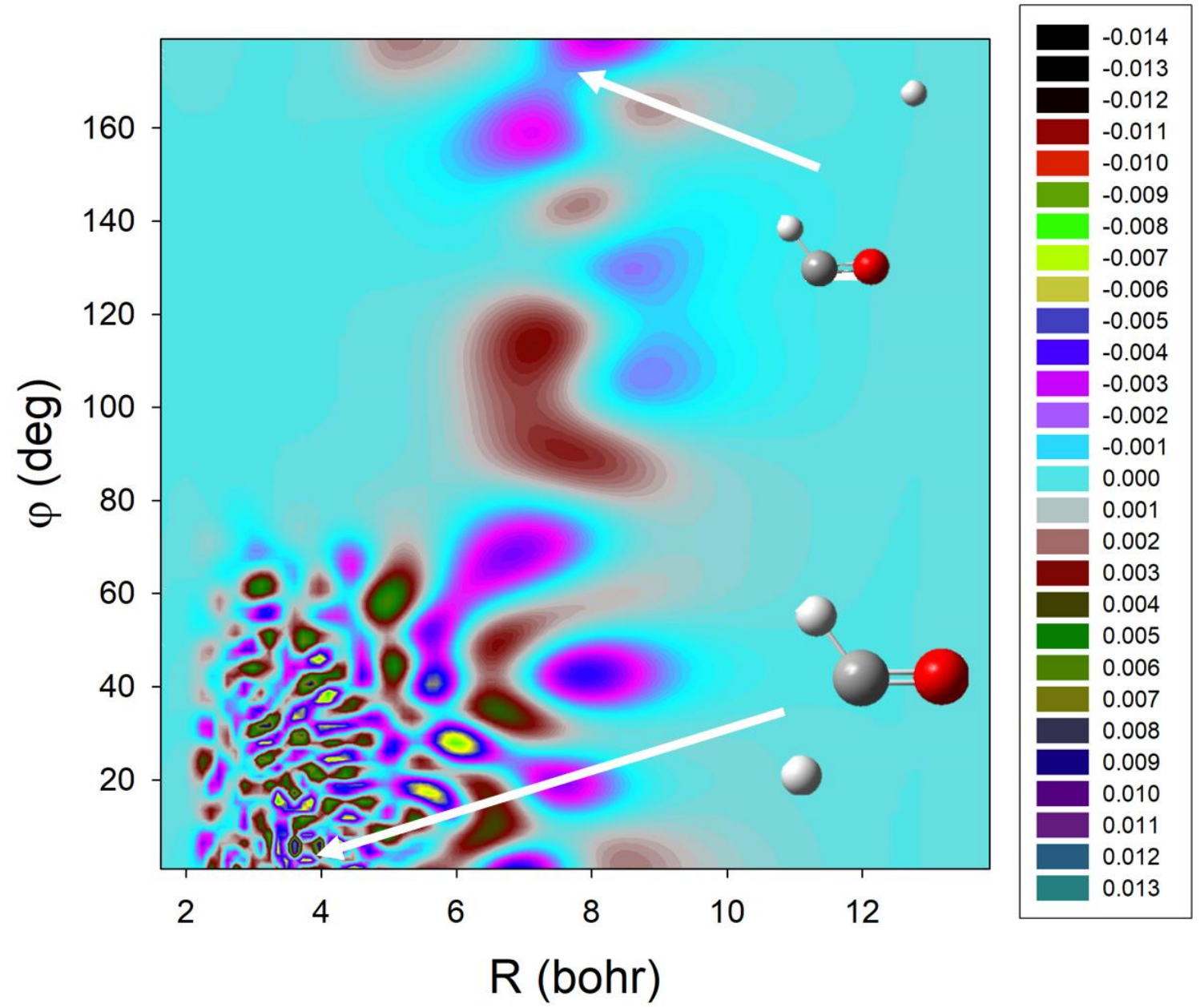

Fig. 6.15 Wavefunction near the radical threshold shown for dihedral angle of $78^{\circ}$. Tight TS and roaming saddle point structures are marked.

Rotational state distributions are usually smooth and highly inverted. They are often explained as a one-to-one mapping of the transition-state bending wavefunction onto product rotation. This mapping of the bound wavefunction on product rotational distributions is known as the well-established rotational reflection principle. ${ }^{231}$ The rotational reflection principle is a general description, and it is too simple to account for all observations. Butenhoff and coworkers concluded in quantum state-correlated experiments that the anisotropy of the potential felt by the $\mathrm{CO}$ is much larger than that felt by the $\mathrm{H}_{2}$ and the coupling of parent zero-point vibrational motions at the transition state to the product angular momentum accounts for the observed product 
distribution widths. ${ }^{261}$ Results from our collaborators indicate that exit channel anisotropy of the PES is responsible for the high rotational excitation seen from the tight-TS pathway, not simply mapping of the bending wavefunction at the TS. Another possibility is thus that the energy is partitioned differently at the TS in the exit channel. If energy is sequestered in another mode, very different parts of the PES would be accessed en route to dissociation and would produce different $j_{\text {CO }}$ distributions. This is consistent with our observed distributions if we compare the tight-TS products $0_{00}$ with $1_{01}$, for example. The former exhibits a relatively large yield of $\mathrm{H}_{2} v=3,4$ in the $j_{\mathrm{CO}}=36$ TED (Fig. 6.4). The $1_{01}$ distribution has a greater yield of $\mathrm{H}_{2} v=0$ to 2. The latter also has less of the lower component of the bimodal rotational distribution. Differential loss to the radical channel should also be considered to explain the observed dynamics, including the similarity of some $j_{\mathrm{CO}}=32$ to 39 TEDs. Initial observations of the relative intensities of ortho and para using $j_{\mathrm{CO}}=36$ and 39 REMPI detection are relatively similar while the $\mathrm{H}$ atom relative intensities are significantly different. Further investigation is needed to quantify this, with consideration given to the lower state energies and beam conditions.

\subsection{Conclusion}

Despite the massive library of data that has been collected on formaldehyde dynamics over decades, this is the first demonstration of quantum effects in. roaming. A symmetry-dependent coupling model that likely contributes to parent rotational-state dependent dynamics was presented. More importantly, resonant coupling between $\mathrm{H}_{2} \mathrm{CO}$ and $\mathrm{H}+\mathrm{HCO}$ decouples the bodyfixed and space-fixed frames, resulting in enhanced roaming that we contribute to the promotion of out-of-plane motion by the resonance. The resonance is not fully understood, but it has the character of an orbiting resonance and modulates the dynamics of three different pathways. An initial framework to understand these effects has been built, but much remains to be done. 
Investigation of systems with strong demonstrated Coriolis coupling such as ozone ${ }^{262}$ or $\mathrm{Cl}_{2} \mathrm{O}_{2}{ }^{263}$ may be fruitful. A more complicated system with active questions regarding roaming fractions, ${ }^{65,264-267}$ acetaldehyde, may benefit from studying the molecule through the lens of the work presented here.

Because there was variation in TEDs from the tight TS pathways, the $2^{1} 4^{1}$ vibrational band must be investigated, where $\mathrm{CO}$ and $\mathrm{H}_{2}$ can only be formed via the tight TS. Imaging of jco can only help to confirm and clarify the dynamics. Similarly, ortho transitions must be further analyzed on all vibrational bands. Different $J$ excitations resulted in very different dynamics. Only $J=1$ and 2 para transitions were studied, and investigation of $J=3$ is necessary to determine any effects that may be occurring with increased rotational energy of para levels.

The massive dataset presented helped clarify the dynamics, but also brought questions to light that need to be answered. For example, when adding linear combinations of $0_{00}$ and $1_{01}$ TEDs, not presented here, they almost exactly match that of 202 . There seems to suggest clusters of similar underlying resonances are involved, likely involving the $\mathrm{H}-\mathrm{H}$ stretching mode. We find other combinations of these TEDs together closely match, perhaps showing where interferences among a few underlying resonances are present. The picture of formaldehyde dynamics is not nearly resolved. A statement by Polanyi in the publication summarizing his Nobel lecture ${ }^{11}$ rings true, "The great epics of reaction dynamics remain to be written." 


\section{APPENDIX A}

Table S1:

Ground state of $\mathrm{C}_{3} \mathrm{H}_{3} \mathrm{Cl}$ ( $\left.{ }^{1} A^{\prime}\right)$ optimized at the $\mathrm{CCSD}(\mathrm{T}) / \operatorname{aug}(\mathrm{Cl})$-cc-pVTZ level

ENERGY $=-575.56728562 \mathrm{Ha}$

$\mathrm{ZPE}=0.04716543 \mathrm{Ha}$

CARTESIAN COORDINATES

$\begin{array}{llll}\mathrm{C} & 0.4367844380 & 0.0000000000 & -2.3874194829 \\ \mathrm{C} & -0.1520975865 & 0.0000000000 & -1.3297318213 \\ \mathrm{C} & -0.8768894695 & 0.0000000000 & -0.0646301285 \\ \mathrm{H} & 0.9580751769 & 0.0000000000 & -3.3142810597 \\ \mathrm{H} & -1.4973763398 & -0.8882609783 & 0.0313868647 \\ \mathrm{H} & -1.4973763398 & 0.8882609783 & 0.0313868647 \\ \mathrm{Cl} & 0.2484590578 & 0.0000000000 & 1.3460919985\end{array}$

HARMONIC VIBRATIONAL FREQUENCIES

Mode Wavenumber

$v_{15} \quad 170.84$

$v_{14} \quad 301.29$

$v_{13} \quad 450.97$

$v_{12} \quad 629.72$

$v_{11} \quad 651.08$

$v_{10} \quad 732.85$

$v_{9} \quad 925.37$

$v_{8} \quad 967.08$

$v_{7} \quad 1204.77$

$v_{6} \quad 1295.56$

v5 $\quad 1482.75$

$v_{4} \quad 2177.33$

$v_{3} \quad 3097.40$

$v_{2} \quad 3151.86$

$v_{1} \quad 3464.36$ 
Table S2:

Triplet state of $\mathrm{C}_{3} \mathrm{H}_{3} \mathrm{Cl}\left({ }^{3} A\right)$ optimized at the $\mathrm{CCSD}(\mathrm{T}) / \mathrm{aug}(\mathrm{Cl})$-cc-pVTZ level

ENERGY $=-575.41942349 \mathrm{Ha}$

$\mathrm{ZPE}=0.04520042 \mathrm{Ha}$

\section{CARTESIAN COORDINATES}

$\begin{array}{llll}\mathrm{C} & -2.3684697260 & -0.2858934013 & -0.2622056316 \\ \mathrm{C} & -1.2545559590 & -0.0353974356 & 0.4424412724 \\ \mathrm{C} & -0.0675225017 & 0.7232010646 & 0.0168929165 \\ \mathrm{H} & -3.2879363268 & -0.8114955612 & -0.0467411998 \\ \mathrm{H} & 0.1635803920 & 1.5502375651 & 0.6854440770 \\ \mathrm{H} & -0.2489357151 & 1.1127077726 & -0.9940194932 \\ \mathrm{Cl} & 1.4084768366 & -0.3067020041 & -0.0662769413\end{array}$

\section{HARMONIC VIBRATIONAL FREQUENCIES}

Mode Wavenumber

$v_{15} \quad 121.77$

$v_{14} \quad 303.47$

$v_{13} \quad 367.11$

$v_{12} \quad 633.14$

$v_{11} \quad 744.94$

$v_{10} \quad 848.76$

$v_{9} \quad 909.52$

$v_{8} \quad 1081.01$

$v_{7} \quad 1163.71$

$v_{6} \quad 1267.70$

$v_{5} \quad 1436.15$

$v_{4} \quad 1627.73$

$v_{3} \quad 2992.13$

$v_{2} \quad 3125.09$

$v_{1} \quad 3218.48$ 


\section{Table S3:}

\section{Triplet minimum depicted in Figure 3.8}

Structure optimized at the SA3-CASSCF(12/10)/6-311+G* level by averaging 3 states of ${ }^{3} A^{\prime \prime}$ symmetry. Geometry optimization performed on the second state $\left(2^{3} A^{\prime \prime}\right)$

\begin{tabular}{llll}
\multicolumn{4}{l}{ CARTESIAN COORDINATES } \\
$\mathrm{C}$ & -2.5726863851 & -0.2750602787 & 0.00000000000 \\
$\mathrm{C}$ & -1.4071124980 & 0.3681859360 & 0.0000000000 \\
$\mathrm{C}$ & -0.1554475076 & 0.9403644055 & 0.0000000000 \\
$\mathrm{H}$ & -2.7229817860 & -1.3405000015 & 0.00000000000 \\
$\mathrm{H}$ & 0.1194439651 & 1.4533595487 & 0.9041760805 \\
$\mathrm{H}$ & 0.1194439651 & 1.4533595487 & -0.9041760805 \\
$\mathrm{Cl}$ & 1.4835392466 & -0.5937261587 & 0.00000000000
\end{tabular}

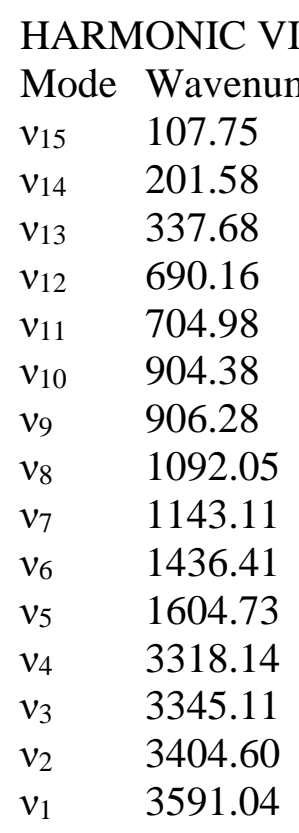

Single-point energies calculated at the SA12-CASSCF(10/11)/aug(Cl)-cc-pVTZ level $\boldsymbol{E}\left(\boldsymbol{X}^{1} A^{\prime}\right)=-574.88799992 \mathrm{Ha}$

$\boldsymbol{E}\left(\boldsymbol{1}^{1} A^{\prime}\right)=-574.75772884 \mathrm{Ha}$

$\boldsymbol{E}\left(2{ }^{1} A^{\prime}\right)=-574.70259682 \mathrm{Ha}$

$\boldsymbol{E}\left(\mathbf{1}^{1} A^{\prime \prime}\right)=-574.76948111 \mathrm{Ha}$

$\boldsymbol{E}\left(2{ }^{1} A^{\prime \prime}\right)=-574.73499153 \mathrm{Ha}$

$\boldsymbol{E}\left(\boldsymbol{3}^{1} A^{\prime \prime}\right)=-574.67084574 \mathrm{Ha}$

$\boldsymbol{E}\left(\mathbf{1}^{3} A^{\prime}\right)=-574.80520730 \mathrm{Ha}$

$\boldsymbol{E}\left(\boldsymbol{2}^{3} A^{\prime}\right)=-574.76441731 \mathrm{Ha}$

$\boldsymbol{E}\left(\boldsymbol{3}^{3} A^{\prime}\right)=-574.72693181 \mathrm{Ha}$

$\boldsymbol{E}\left(\mathbf{1}^{3} A^{\prime \prime}\right)=-574.77697624 \mathrm{Ha}$

$\boldsymbol{E}\left(2^{3} A^{\prime \prime}\right)=-574.75962547 \mathrm{Ha}$ (energy depicted by an asterisk in Fig. 3.8)

$\boldsymbol{E}\left(\boldsymbol{3}^{3} A^{\prime \prime}\right)=-574.67301351 \mathrm{Ha}$ 


\section{APPENDIX B}

Two dissociation pathways for propargyl chloride through which it is dissociated to linear $\mathrm{C}_{3} \mathrm{H}_{2}$ and $\mathrm{HCl}$. Our calculations show a third reaction pathway with higher barrier energy in which cyclic $\mathrm{C}_{3} \mathrm{H}_{2}$ is produced. The geometry of structures of transition states were confirmed by intrinsic reaction coordinate (IRC) calculations. The optimized geometry of reactant, transition states and products with these two levels of theory as well as IRC pathway of the roaming transition state are given below:

Pathway 1:

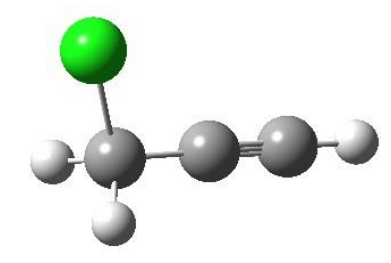

reactant

Z-matrix:

DFT-WB97xD/cc-pvDz

$\begin{array}{lrrr}\mathrm{H} & -1.10675600 & 0.69537000 & 0.00000100 \\ \mathrm{H} & 2.30229500 & 3.06458200 & -0.89525300 \\ \mathrm{H} & 2.30244100 & 3.06453100 & 0.89483100 \\ \mathrm{C} & -0.15911100 & 1.19280200 & -0.00006300 \\ \mathrm{C} & 0.91105500 & 1.75360800 & -0.00013500 \\ \mathrm{C} & 2.19074300 & 2.44408100 & -0.00022000 \\ \mathrm{Cl} & 3.58198300 & 1.28361600 & -0.00036600\end{array}$

CBS-QB3:

$\begin{array}{lrrr}\mathrm{H} & -3.26549500 & -0.89171800 & 0.00024100 \\ \mathrm{H} & 0.13799600 & 1.45170600 & -0.88870800 \\ \mathrm{H} & 0.13814100 & 1.45165500 & 0.88876800 \\ \mathrm{C} & -2.32341200 & -0.40018300 & 0.00017800 \\ \mathrm{C} & -1.25506400 & 0.14685000 & 0.00010700 \\ \mathrm{C} & 0.01710900 & 0.83366200 & 0.00002200 \\ \mathrm{Cl} & 1.43279800 & -0.32315400 & -0.00012600\end{array}$




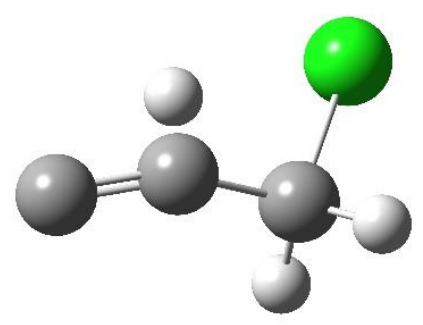

Transition state1

Z-matrix:

DFT-WB97xD/cc-pvDz

$\begin{array}{lrrr}\mathrm{H} & -0.49341600 & -0.35552000 & -0.57299600 \\ \mathrm{H} & 1.32994300 & 1.50891500 & -1.05681900 \\ \mathrm{H} & 1.42681300 & 1.81380800 & 0.70611000 \\ \mathrm{C} & -1.16235100 & 0.25778300 & 0.78567300 \\ \mathrm{C} & -0.05785800 & 0.41954100 & 0.14549200 \\ \mathrm{C} & 1.25985500 & 1.05621700 & -0.06403500 \\ \mathrm{Cl} & 2.61060600 & -0.13740000 & 0.05536900\end{array}$

\section{CBS-QB3:}

$\begin{array}{lrrr}\mathrm{H} & 1.59833500 & -0.50873800 & 1.09464500 \\ \mathrm{H} & -0.09924200 & 1.49734100 & 0.80650900 \\ \mathrm{H} & 0.04831000 & 1.33221500 & -0.95893900 \\ \mathrm{C} & 2.43406700 & -0.43654900 & -0.21305300 \\ \mathrm{C} & 1.29942100 & 0.04041300 & 0.12709500 \\ \mathrm{C} & 0.04652700 & 0.79701000 & -0.01250300 \\ \mathrm{Cl} & -1.42514700 & -0.27800400 & -0.02067300\end{array}$


Z-matrix:

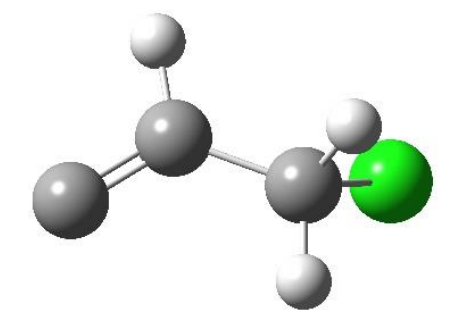

Product 1

DFT-WB97xD/cc-pvDz

$\begin{array}{lrrr}\mathrm{H} & -1.47886600 & 0.89556200 & 0.01832900 \\ \mathrm{H} & 0.90022800 & 1.70365100 & 0.70735900 \\ \mathrm{H} & 0.49543800 & 1.36839100 & 2.41917200 \\ \mathrm{C} & -2.11657100 & 1.47172400 & 1.83716000 \\ \mathrm{C} & -1.14074500 & 1.13404300 & 1.03747300 \\ \mathrm{C} & 0.31665200 & 1.07101600 & 1.38256000 \\ \mathrm{Cl} & 0.95946400 & -0.60666400 & 1.19376200\end{array}$

CBS-QB3:

$\begin{array}{lrrr}\mathrm{H} & 1.35452200 & -0.59775700 & 1.33066600 \\ \mathrm{H} & -0.04408600 & 1.50768300 & 0.76453500 \\ \mathrm{H} & 0.23143900 & 1.20735500 & -0.96770400 \\ \mathrm{C} & 2.33843300 & -0.33230200 & -0.39873500 \\ \mathrm{C} & 1.30174900 & -0.10798100 & 0.35359400 \\ \mathrm{C} & 0.11823600 & 0.74307900 & 0.00802900 \\ \mathrm{Cl} & -1.41719900 & -0.23141500 & -0.05322500\end{array}$




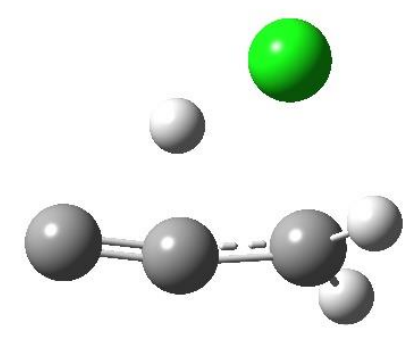

Transition state2

Z-matrix:

DFT-WB97xD/cc-pvDz

$\begin{array}{lrrr}\mathrm{H} & 0.37675000 & 1.95772200 & 0.47580800 \\ \mathrm{H} & 1.82832100 & 3.92831500 & -0.47327700 \\ \mathrm{H} & 1.78280200 & 3.97736400 & 1.38061700 \\ \mathrm{C} & -1.26715300 & 2.96945700 & 0.40378400 \\ \mathrm{C} & -0.08419500 & 3.42442200 & 0.42142500 \\ \mathrm{C} & 1.29710600 & 3.67884900 & 0.44844200 \\ \mathrm{Cl} & 1.81272700 & 1.52027300 & 0.52087000\end{array}$

CBS-QB3:

$\begin{array}{lrrr}\mathrm{H} & 0.24622600 & -0.72641700 & 0.45444700 \\ \mathrm{H} & 0.03349400 & 1.60611300 & 0.66064200 \\ \mathrm{H} & 1.28374600 & 1.89936200 & -0.69242200 \\ \mathrm{C} & 2.09524600 & -1.02137300 & -0.08217800 \\ \mathrm{C} & 1.21217100 & -0.08609200 & 0.11330700 \\ \mathrm{C} & 0.79654300 & 1.22009600 & 0.00178900 \\ \mathrm{Cl} & -1.54042500 & -0.20322600 & -0.03648100\end{array}$



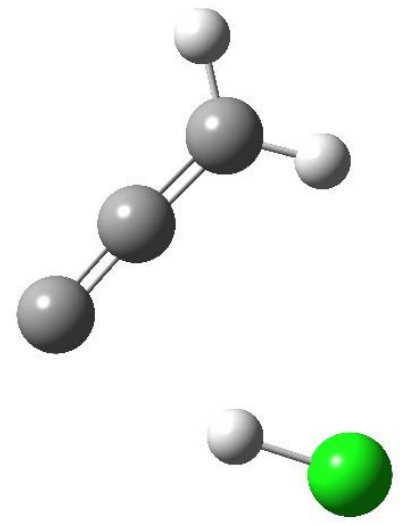

Complex

Z-matrix:

DFT-WB97xD/cc-pvDz

$\begin{array}{lrrr}\mathrm{H} & 3.10388900 & 1.82355900 & -0.14473200 \\ \mathrm{H} & 1.82457000 & 3.15317800 & 0.18162300 \\ \mathrm{C} & 0.27059000 & 0.19129000 & 0.18712800 \\ \mathrm{C} & 1.12701700 & 1.15535600 & 0.12676500 \\ \mathrm{C} & 2.06229300 & 2.09457700 & 0.05126700 \\ \mathrm{H} & 2.17748600 & -1.10667000 & -0.23982900 \\ \mathrm{Cl} & 3.39580400 & -1.50792400 & -0.48038500\end{array}$

\section{CBS-QB3:}

$\begin{array}{lrrr}\mathrm{H} & -0.91404800 & 1.52938600 & -0.92541900 \\ \mathrm{H} & -0.91331600 & 1.52889300 & 0.92538400 \\ \mathrm{C} & -2.79045800 & -1.03847400 & 0.00002100 \\ \mathrm{C} & -2.04769600 & 0.00844600 & 0.00002200 \\ \mathrm{C} & -1.25694700 & 1.07051000 & -0.00000300 \\ \mathrm{H} & 1.58630800 & -1.26568700 & -0.00055900 \\ \mathrm{Cl} & 2.16539200 & -0.11973400 & 0.00002100\end{array}$


Pathway 2:

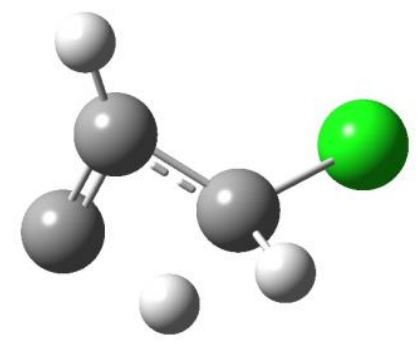

Transition state 2

Z-matrix:

DFT-WB97xD/cc-pvDz

$\begin{array}{lrrr}\mathrm{H} & -4.27598500 & 0.84154600 & -0.83078000 \\ \mathrm{H} & -2.24304300 & 2.70854200 & 0.38087300 \\ \mathrm{H} & -3.57298700 & 2.44849900 & 1.68777300 \\ \mathrm{C} & -4.61281700 & 1.97933700 & 1.09616200 \\ \mathrm{C} & -4.02201000 & 1.40736400 & 0.05294600 \\ \mathrm{C} & -2.83351300 & 1.86156100 & 0.73671200 \\ \mathrm{Cl} & -1.78946600 & 0.67933900 & 1.58049900\end{array}$

CBS-QB3:

$\begin{array}{lrrr}\mathrm{H} & -1.70010500 & 1.62735200 & -0.22351700 \\ \mathrm{H} & -0.21015900 & -0.14616900 & 1.65885400 \\ \mathrm{H} & -0.77771900 & -1.23802300 & 0.24962400 \\ \mathrm{C} & -1.74437100 & -0.61946400 & -0.38130300 \\ \mathrm{C} & -1.38406400 & 0.61369000 & -0.06448900 \\ \mathrm{C} & -0.28342600 & -0.06785700 & 0.57846600 \\ \mathrm{Cl} & 1.36230300 & 0.01168400 & -0.14594100\end{array}$


Z-matrix:

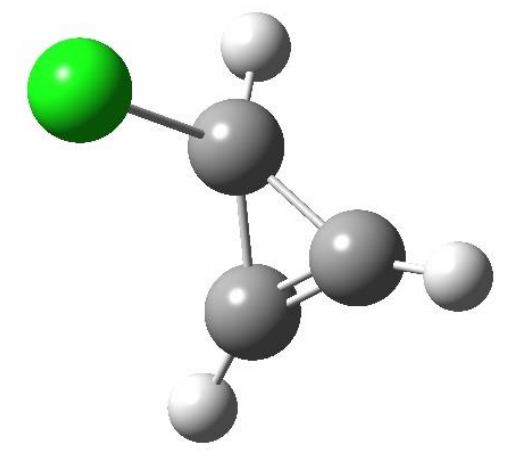

\section{DFT-WB97xD/cc-pvDz}

$\begin{array}{lrrr}\mathrm{H} & 1.85869000 & -1.59642600 & -0.49060700 \\ \mathrm{H} & 0.22925200 & 0.00000300 & 1.66331500 \\ \mathrm{H} & 1.85874700 & 1.59639500 & -0.49062300 \\ \mathrm{C} & 1.43553500 & 0.65476700 & -0.16066600 \\ \mathrm{C} & 1.43554200 & -0.65476500 & -0.16066800 \\ \mathrm{C} & 0.34002700 & 0.00000000 & 0.57768700 \\ \mathrm{Cl} & -1.36548900 & 0.00000100 & -0.13060000\end{array}$

\section{CBS-QB3:}

$\begin{array}{lrrr}\mathrm{H} & 1.85233900 & -1.58675500 & -0.48545400 \\ \mathrm{H} & 0.23098700 & 0.00000700 & 1.65374500 \\ \mathrm{H} & 1.85243000 & 1.58669400 & -0.48551000 \\ \mathrm{C} & 1.43266500 & 0.65027900 & -0.16058300 \\ \mathrm{C} & 1.43267500 & -0.65028100 & -0.16057700 \\ \mathrm{C} & 0.34018400 & 0.00001100 & 0.57514700 \\ \mathrm{Cl} & -1.36287700 & 0.00000000 & -0.12980600\end{array}$




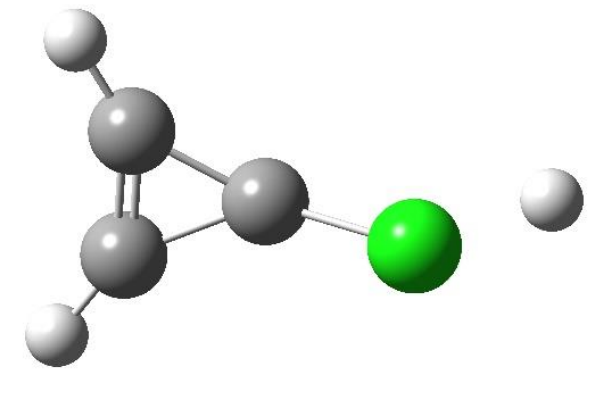

Transition state 3

Z-matrix:

DFT-WB97xD/cc-pvDz

$\begin{array}{lrrr}\mathrm{H} & -2.54715400 & -0.86054800 & 0.33458300 \\ \mathrm{H} & 1.92848700 & -0.49885500 & 2.25159200 \\ \mathrm{H} & -2.75611200 & 0.55901900 & 3.13212200 \\ \mathrm{C} & -2.12702500 & 0.03604900 & 2.42112400 \\ \mathrm{C} & -2.03908000 & -0.56120500 & 1.24395900 \\ \mathrm{C} & -0.80518400 & -0.45452500 & 2.02538300 \\ \mathrm{Cl} & 0.45464400 & -1.31978700 & 2.55834000\end{array}$

CBS-QB3:

$\begin{array}{lrrr}\mathrm{H} & 2.08527400 & -1.56847400 & -0.17933600 \\ \mathrm{H} & -2.34421800 & 0.00000000 & 1.24650000 \\ \mathrm{H} & 2.08527500 & 1.56847400 & -0.17933500 \\ \mathrm{C} & 1.52945900 & 0.65811100 & -0.02582400 \\ \mathrm{C} & 1.52945900 & -0.65811100 & -0.02582400 \\ \mathrm{C} & 0.28286800 & 0.00000000 & 0.36101400 \\ \mathrm{Cl} & -1.28688500 & 0.00000000 & -0.16141300\end{array}$




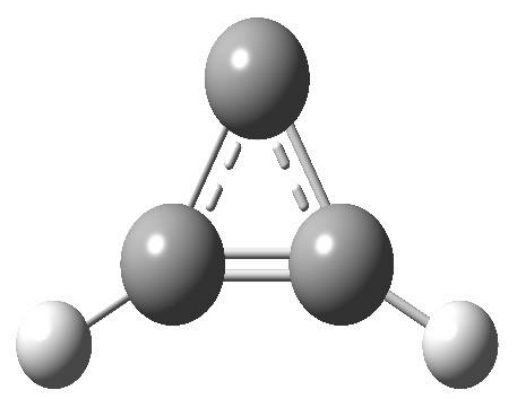

Z-matrix:

\author{
Cyclic $\mathrm{C}_{3} \mathrm{H}_{2}$
}

DFT-WB97xD/cc-pvDz

$\begin{array}{lrrr}\mathrm{C} & -0.66501000 & -0.31962400 & -0.00000100 \\ \mathrm{C} & 0.66501000 & -0.31962400 & 0.00000300 \\ \mathrm{C} & 0.00000000 & 0.93885000 & -0.00000400 \\ \mathrm{H} & -1.60452800 & -0.86385300 & -0.00000100 \\ \mathrm{H} & 1.60452800 & -0.86385300 & 0.00000800\end{array}$

CBS-QB3:

$\begin{array}{lrrc}\mathrm{C} & -0.66131300 & -0.32400600 & -0.00000100 \\ \mathrm{C} & 0.66131300 & -0.32400600 & 0.00000300 \\ \mathrm{C} & 0.00000000 & 0.93579800 & -0.00000400 \\ \mathrm{H} & -1.59649300 & -0.86335700 & -0.00000100 \\ \mathrm{H} & 1.59649300 & -0.86335700 & 0.00000800\end{array}$


Pathway 3:

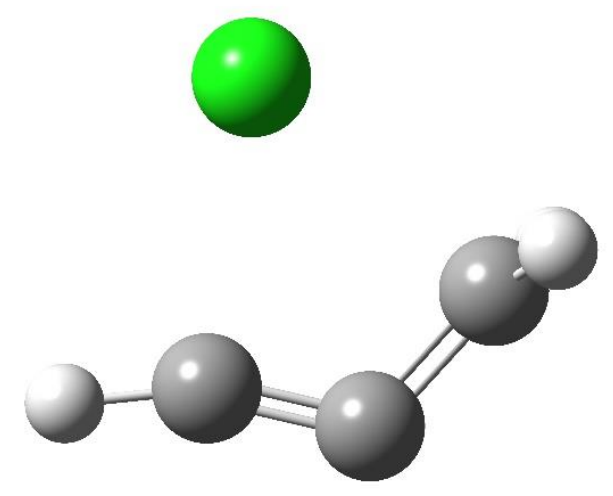

Transition state 1

Z-matrix:

DFT-WB97xD/cc-pvDz

$\begin{array}{lrrr}\mathrm{H} & 1.40735400 & -0.02910800 & -0.03186000 \\ \mathrm{H} & 5.09517000 & 1.15161800 & -0.99786100 \\ \mathrm{H} & 5.12151700 & 1.12259200 & 0.86542300 \\ \mathrm{C} & 2.47811400 & 0.08424200 & -0.04526400 \\ \mathrm{C} & 3.70606300 & -0.20038300 & -0.06720500 \\ \mathrm{C} & 4.63915800 & 0.81679200 & -0.06457500 \\ \mathrm{Cl} & 2.81427700 & 2.41978600 & -0.01380600\end{array}$

CBS-QB3:

$\begin{array}{lrrr}\mathrm{H} & 0.59972900 & 2.31456000 & 0.00000000 \\ \mathrm{H} & 1.02663200 & -1.68737400 & 0.92923800 \\ \mathrm{H} & 1.02663300 & -1.68737400 & -0.92923800 \\ \mathrm{C} & 0.87148000 & 1.28348500 & 0.00000000 \\ \mathrm{C} & 1.43172000 & 0.18092400 & 0.00000000 \\ \mathrm{C} & 1.09649200 & -1.13256500 & 0.00000000 \\ \mathrm{Cl} & -1.35595000 & -0.05475700 & 0.00000000\end{array}$




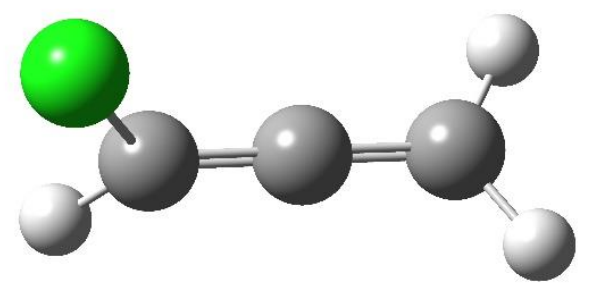

Z-matrix:

Allene chloride

DFT-WB97xD/cc-pvDz

$\begin{array}{lrrr}\mathrm{H} & -0.51881100 & -0.98888200 & -0.00099000 \\ \mathrm{H} & 3.33130500 & -1.38711200 & -0.99640200 \\ \mathrm{H} & 3.35726500 & -1.41727400 & 0.87337800 \\ \mathrm{C} & 0.35300400 & -0.33839800 & -0.00261000 \\ \mathrm{C} & 1.58534300 & -0.76879400 & -0.02683400 \\ \mathrm{C} & 2.81545400 & -1.21234800 & -0.05110700 \\ \mathrm{Cl} & -0.03999600 & 1.37062900 & 0.03051000\end{array}$

\section{CBS-QB3:}

$\begin{array}{lrrr}\mathrm{H} & -0.25785000 & 1.78081200 & 0.00042000 \\ \mathrm{H} & 2.85294000 & -0.52831300 & 0.92747700 \\ \mathrm{H} & 2.85333100 & -0.52733000 & -0.92750600 \\ \mathrm{C} & -0.05647000 & 0.71864200 & 0.00000600 \\ \mathrm{C} & 1.13303700 & 0.19814100 & 0.00000500 \\ \mathrm{C} & 2.33243200 & -0.30767800 & -0.00000700 \\ \mathrm{Cl} & -1.52367200 & -0.25763500 & -0.00002400\end{array}$




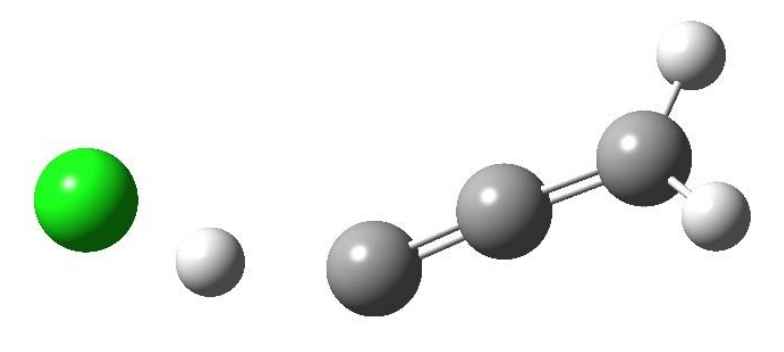

Z-matrix:

Transition state 2

DFT-WB97xD/cc-pvDz

$\begin{array}{lrrr}\mathrm{H} & -1.29895500 & -3.04765600 & -0.17010800 \\ \mathrm{H} & 2.91524500 & -1.51757300 & -0.76720200 \\ \mathrm{H} & 2.88257300 & -1.85515700 & 1.07345100 \\ \mathrm{C} & 0.14310100 & -3.21138000 & -0.17500300 \\ \mathrm{C} & 1.22603300 & -2.54833000 & -0.03459100 \\ \mathrm{C} & 2.40030100 & -1.94403300 & 0.09701200 \\ \mathrm{Cl} & -2.44715400 & -2.19938600 & -0.03443000\end{array}$

CBS-QB3:

$\begin{array}{lrrr}\mathrm{H} & 0.99611800 & 0.53172100 & -0.00000200 \\ \mathrm{H} & -3.44069600 & -0.59030200 & -0.92835000 \\ \mathrm{H} & -3.44070400 & -0.59027700 & 0.92835400 \\ \mathrm{C} & -0.58293300 & 0.76290800 & -0.00000300 \\ \mathrm{C} & -1.71486400 & 0.18351700 & -0.00000100 \\ \mathrm{C} & -2.92338500 & -0.35778500 & 0.00000100 \\ \mathrm{Cl} & 2.18896300 & -0.16958700 & 0.00000100\end{array}$


IRC pathway (for roaming transition state):

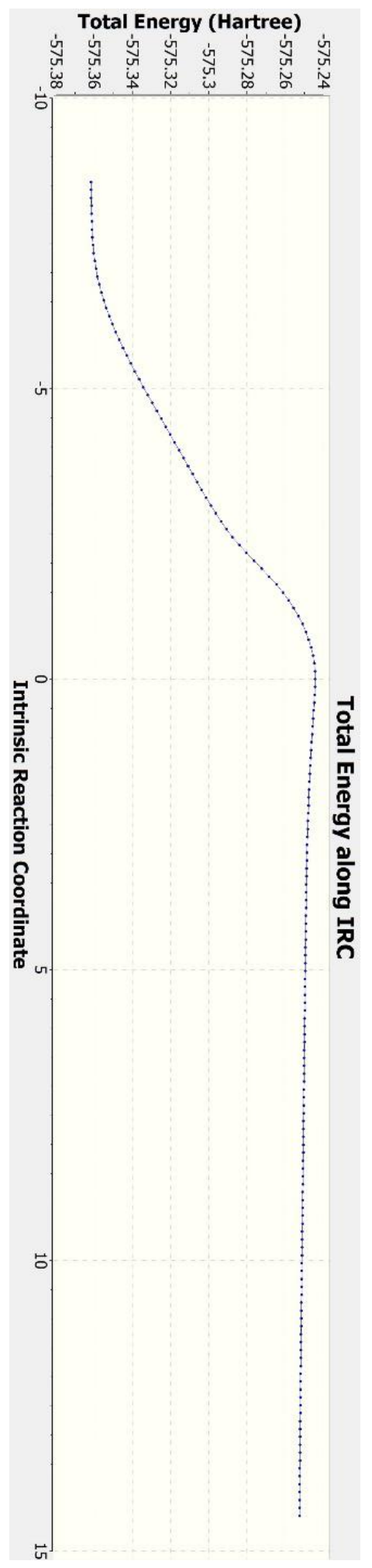




\section{APPENDIX C}

DC slice images for the different transitions used to exited formaldehyde are included here, with the rotational line indicated at the top of the page. The jco imaged is also indicated. 
$2^{1} 6^{1}$

${ }^{\mathrm{r}} \mathrm{R}_{0,0}(0) \rightarrow 1_{11}$

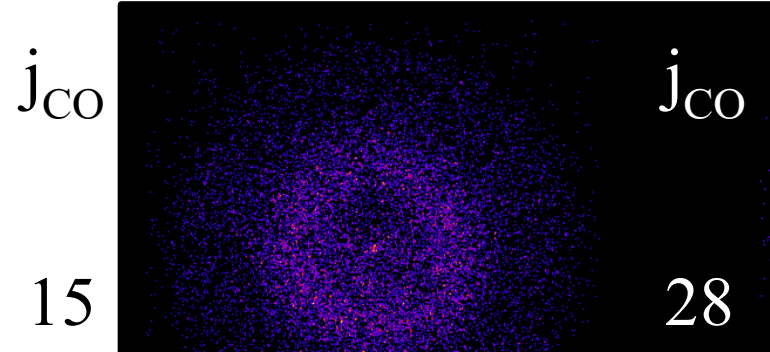

16

32

20

37

23

44 
$2^{1} 6^{1}$

${ }^{\mathrm{r}} \mathrm{R}_{0,0}(0) \rightarrow 1_{11}$

$j_{\text {CO }}$
45

46

50

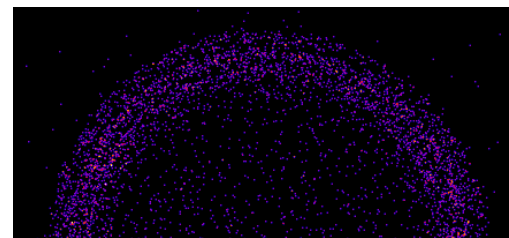

51 
$2^{1} 6^{1}$

${ }^{\mathrm{r}} \mathrm{Q}_{0,1}(1) \rightarrow 1_{10}$

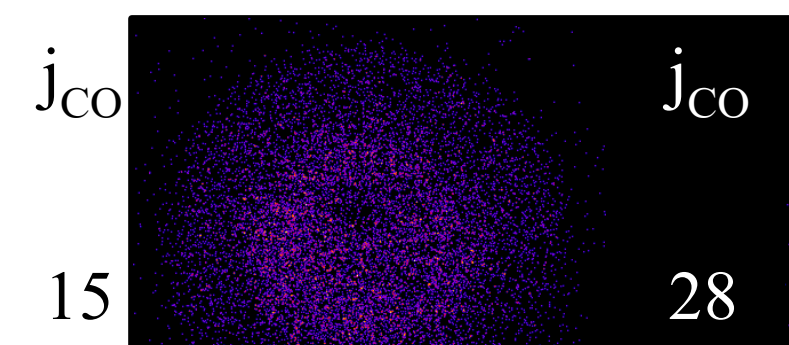

16

32

20

37

23

44 
$2^{1} 6^{1}$

${ }^{\mathrm{r}} \mathrm{Q}_{0,1}(1) \rightarrow 1_{10}$

$\mathrm{j}_{\mathrm{CO}}$
45

46

50

51

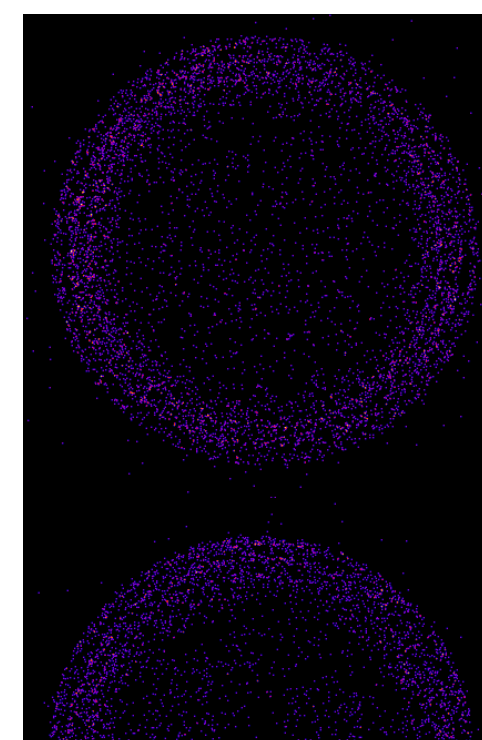

46 
$2^{1} 6^{1}$

${ }^{\mathrm{r}} \mathrm{Q}_{0,2}(2) \rightarrow 2_{11}$

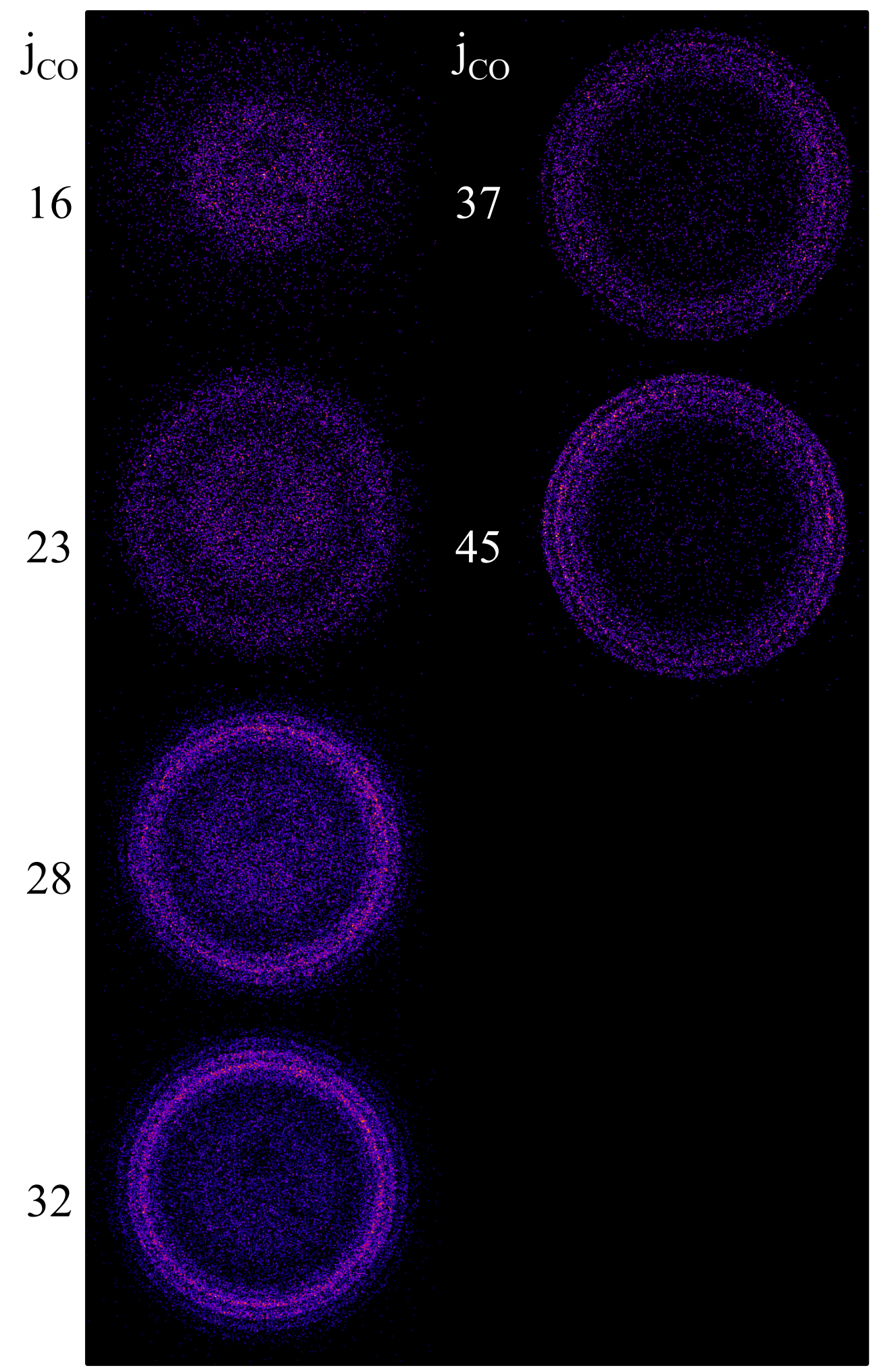


$2^{1} 6^{1} \quad{ }^{\mathrm{r}} \mathrm{R}_{0,1}(1) \rightarrow 2_{12}$

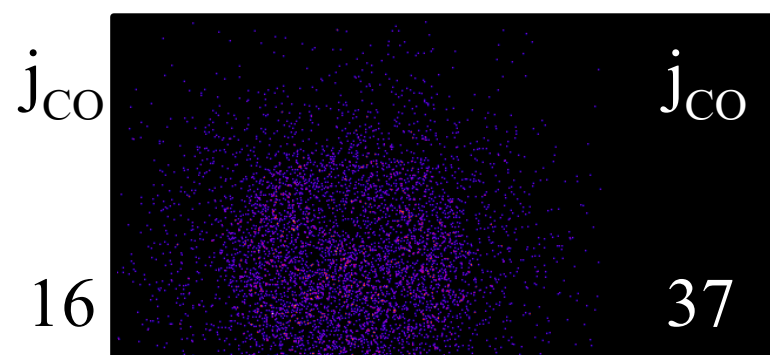

23

45 
$2^{1} 4^{3}$

${ }^{\mathrm{r}} \mathrm{R}_{0,0}(0) \rightarrow 1_{11}$

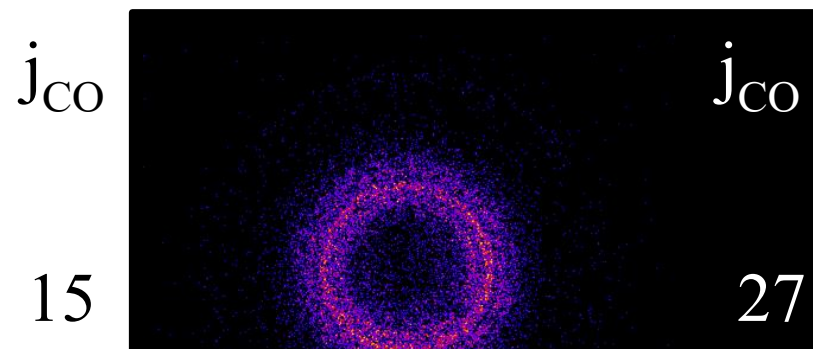

16

28

22

29

23

32 
$2^{1} 4^{3}$

${ }^{\mathrm{r}} \mathrm{R}_{0,0}(0) \rightarrow 1_{11}$

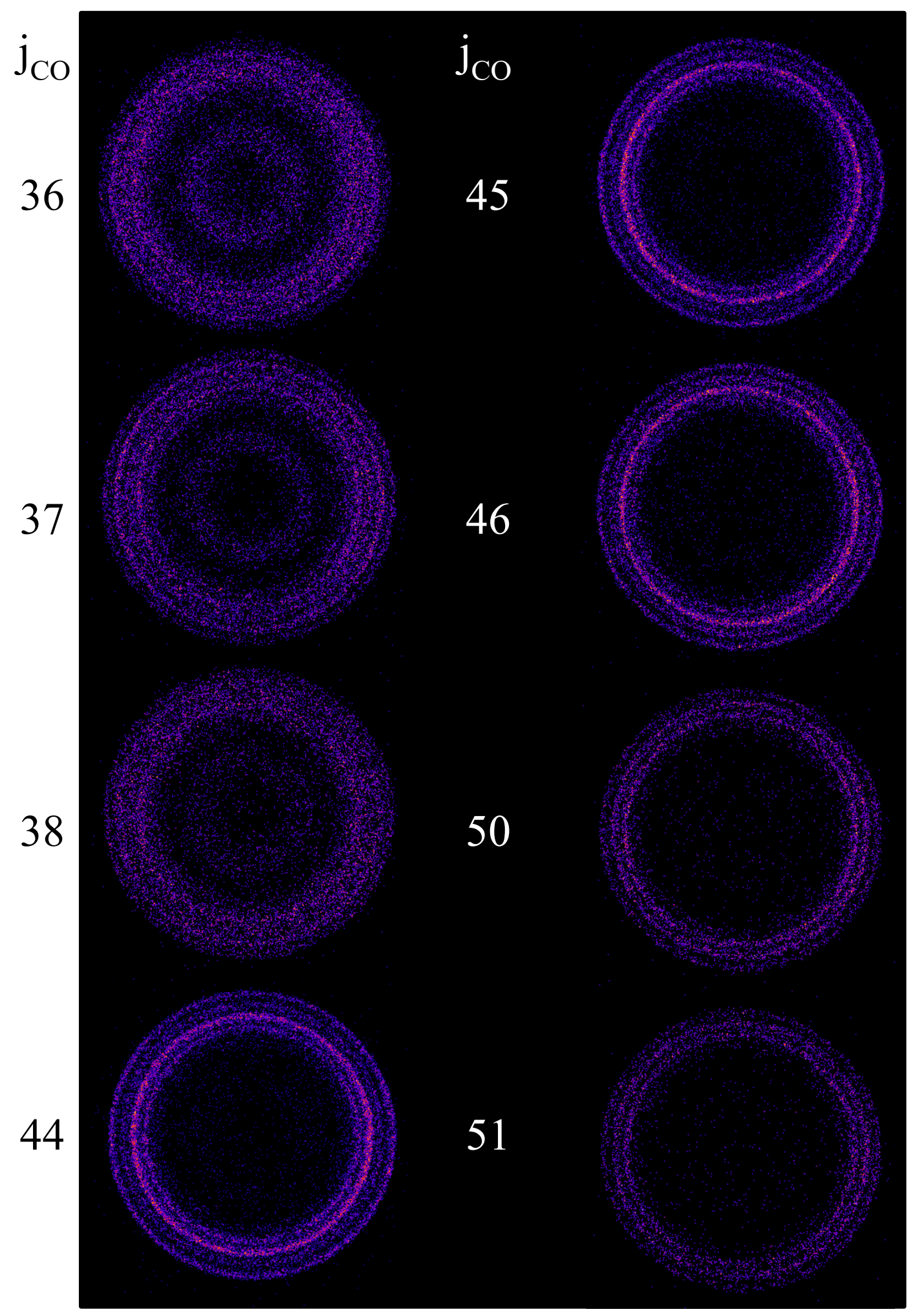


$2^{1} 4^{3}$

${ }^{\mathrm{r}} \mathrm{R}_{0,0}(0) \rightarrow 1_{11}$

54

55

60

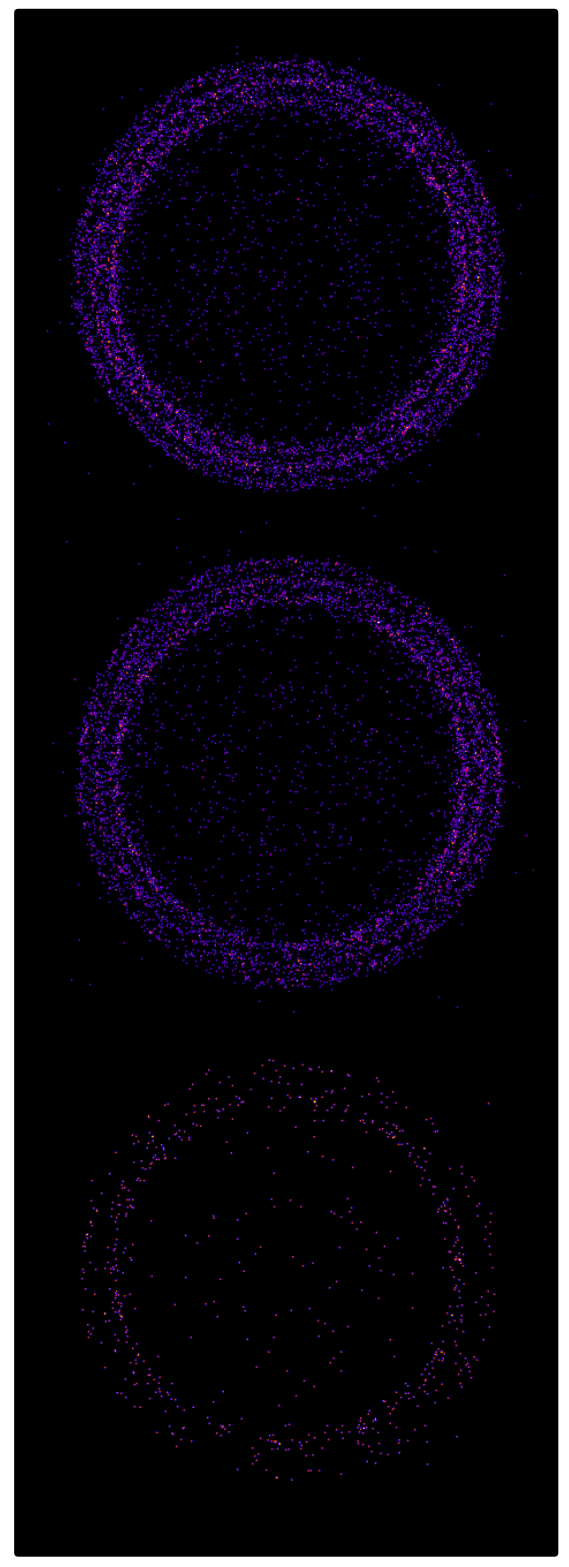


$2^{1} 4^{3}$

${ }^{\mathrm{r}} \mathrm{Q}_{0,1}(1) \rightarrow 1_{10}$

$\mathrm{j}_{\mathrm{CO}}$

15

27

16

28

22

29

23

32 
$2^{1} 4^{3}$

${ }^{\mathrm{r}} \mathrm{Q}_{0,1}(1) \rightarrow 1_{10}$

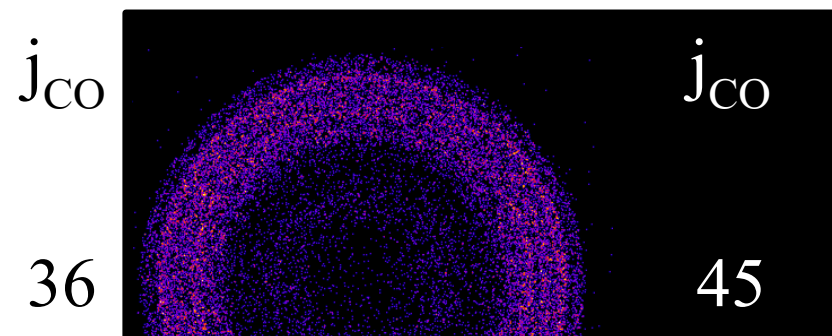

37

46

38

50

44

51 
$2^{1} 4^{3}$

${ }^{\mathrm{r}} \mathrm{Q}_{0,1}(1) \rightarrow 1_{10}$

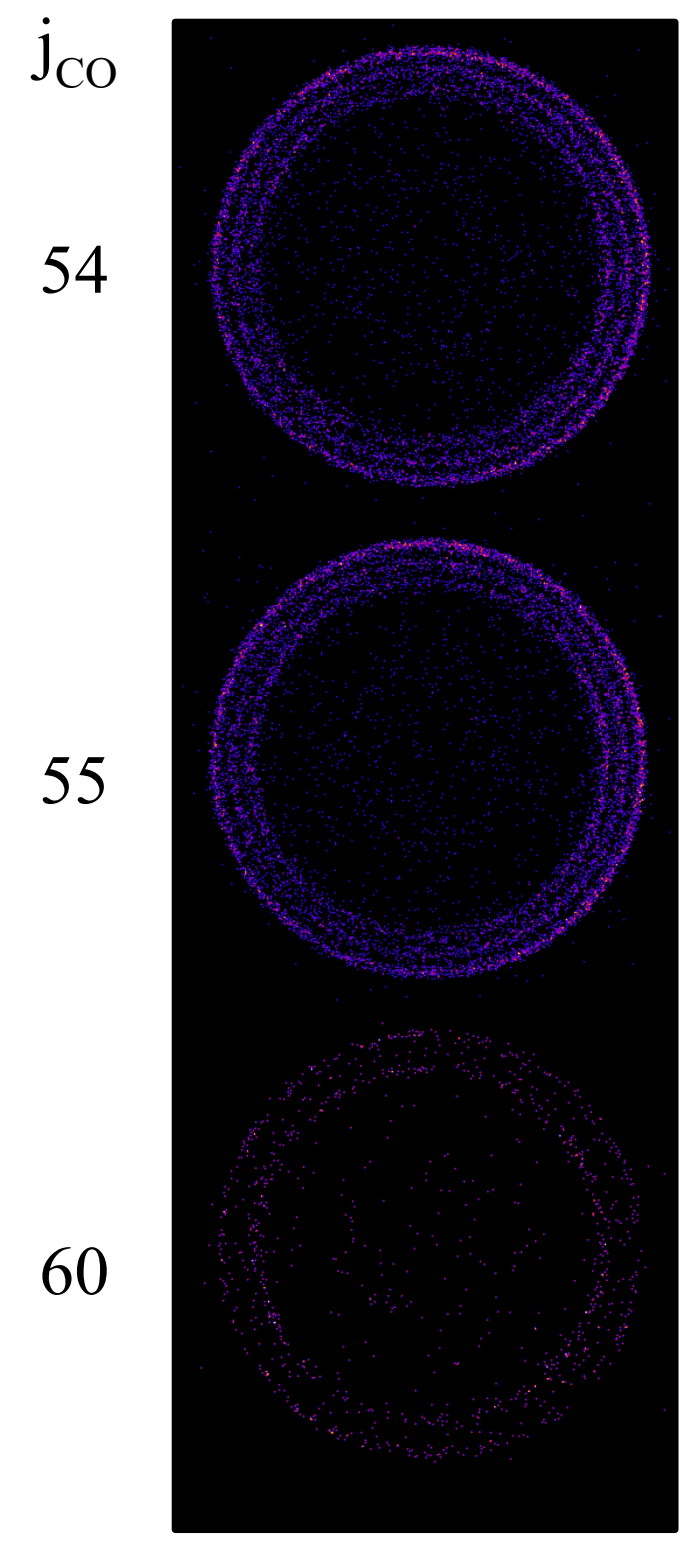


$2^{1} 4^{3}$

${ }^{\mathrm{r}} \mathrm{Q}_{0,2}(2) \rightarrow 2_{11}$

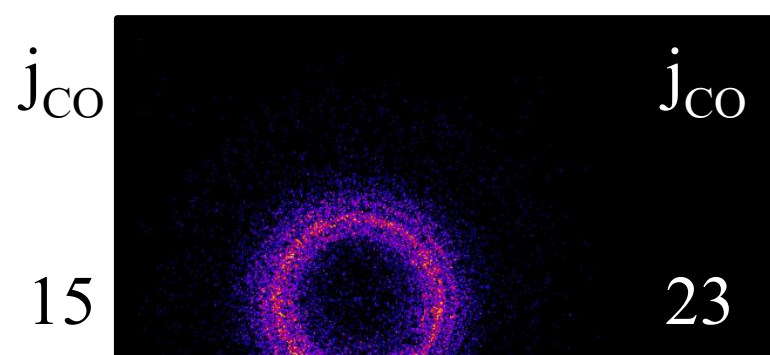

16

24

20

27

22

28 
$2^{1} 4^{3} \quad{ }^{\mathrm{r}} \mathrm{Q}_{0,2}(2) \rightarrow 2_{11}$

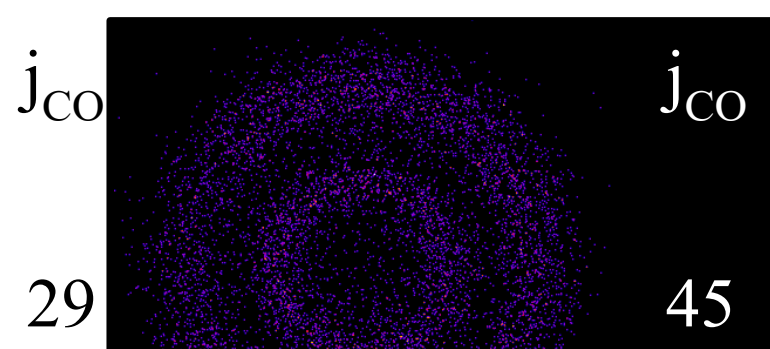

32

50

33

55

37

60 
$2^{1} 4^{3} \quad{ }^{\mathrm{r}} \mathrm{R}_{0,1}(1) \rightarrow 2_{12}$

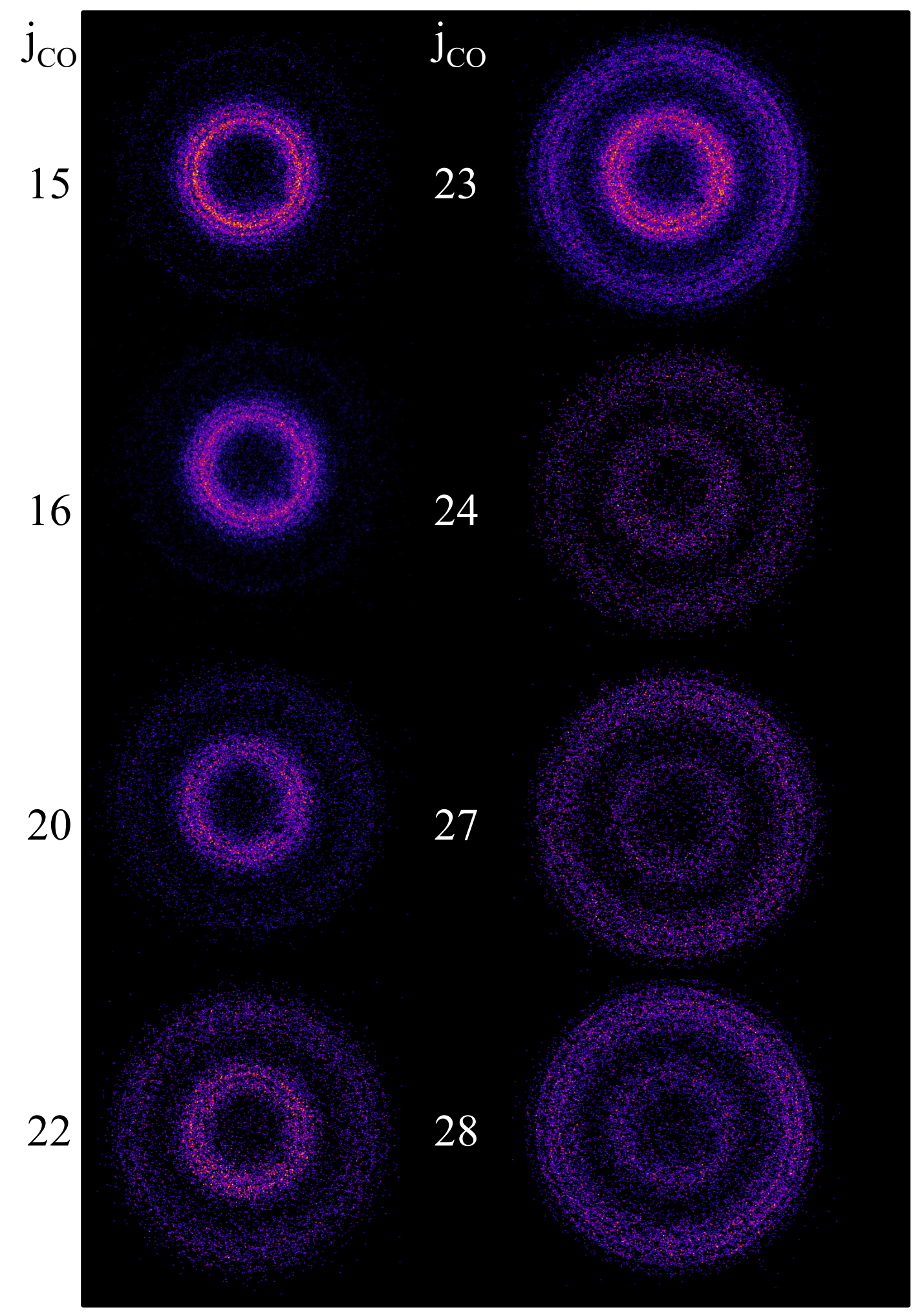


$2^{1} 4^{3} \quad{ }^{\mathrm{r}} \mathrm{R}_{0,1}(1) \rightarrow 2_{12}$

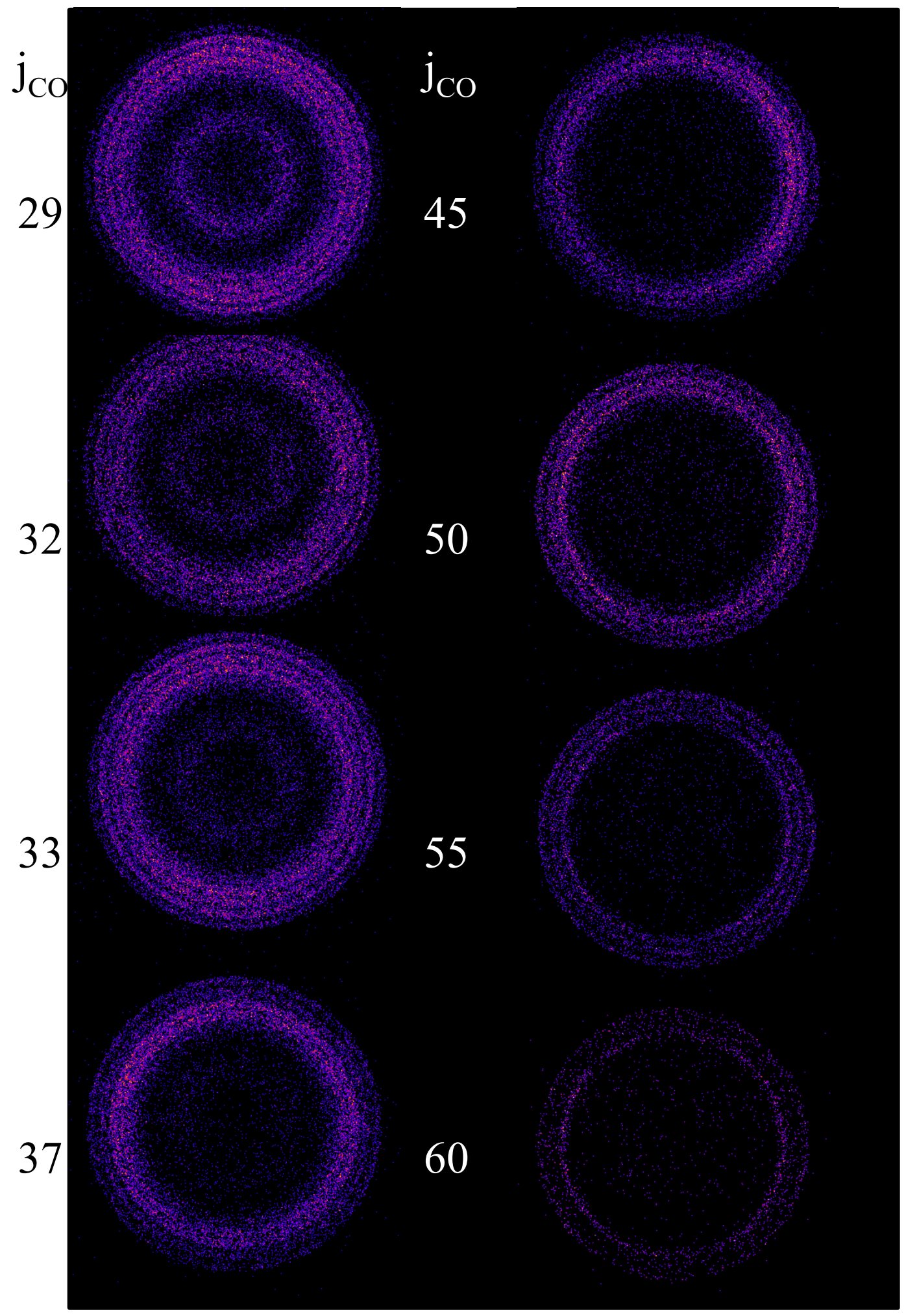


$2^{1} 4^{3}$

${ }^{\mathrm{p}} \mathrm{P}_{1,1}(1) \rightarrow 0_{00}$

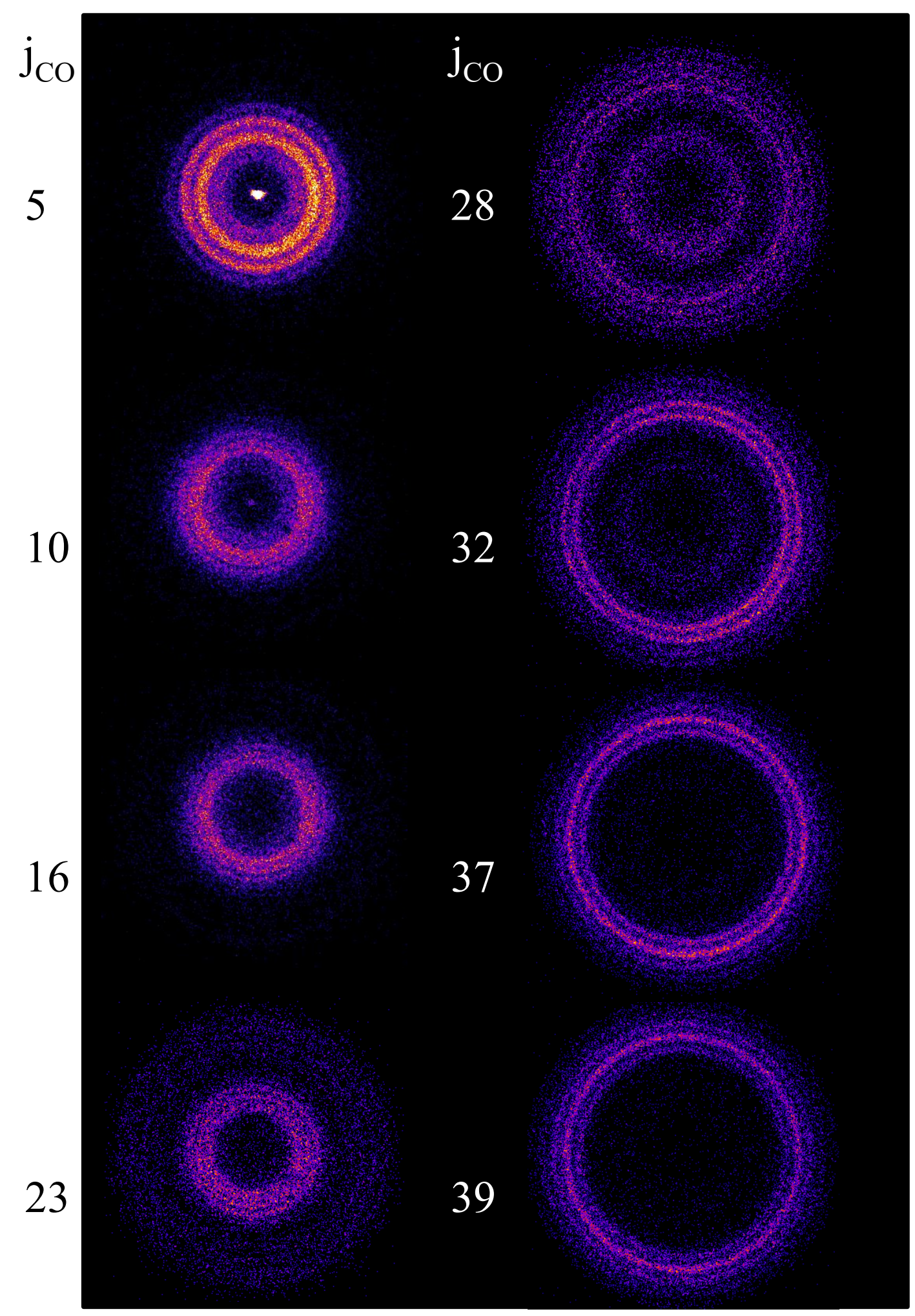




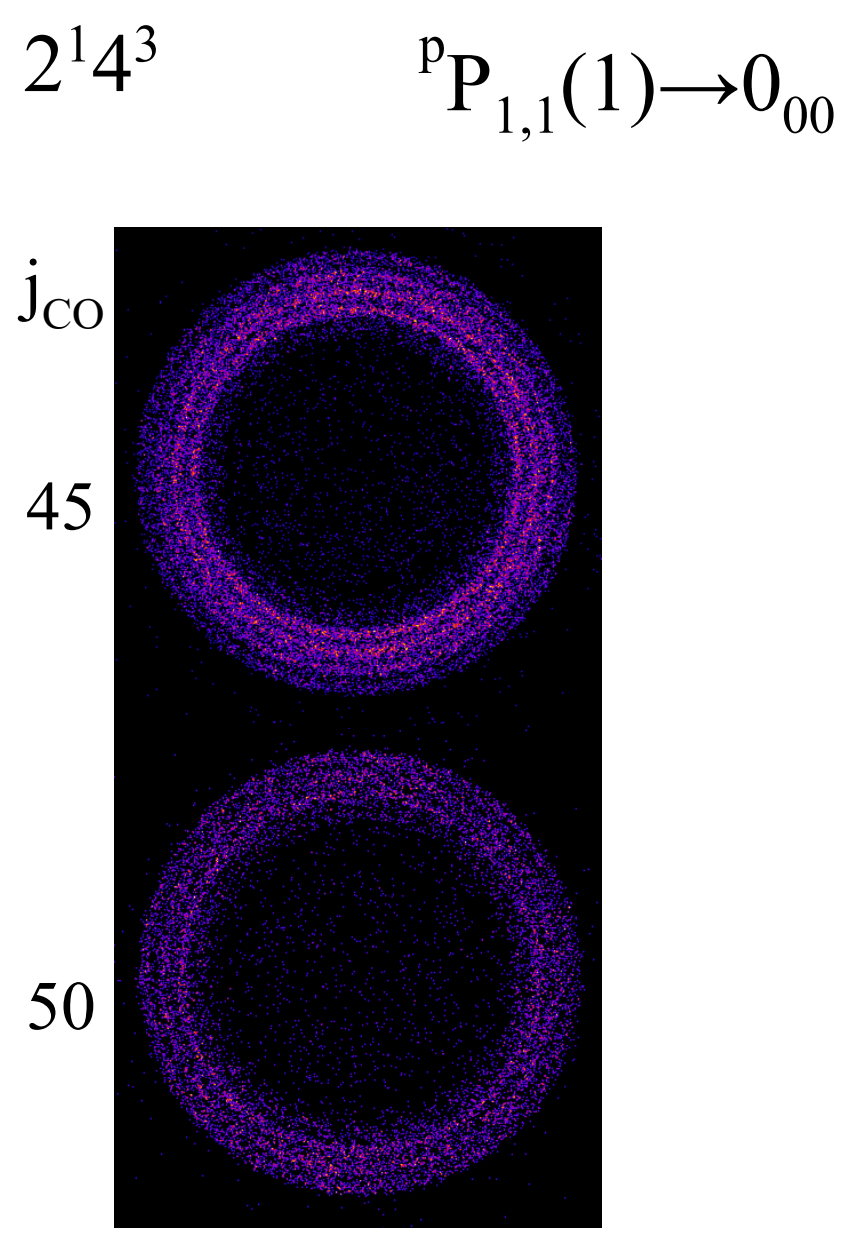


$2^{1} 4^{3}$

$$
{ }^{\mathrm{p}} \mathrm{Q}_{1,0}(1) \rightarrow 1_{01}
$$

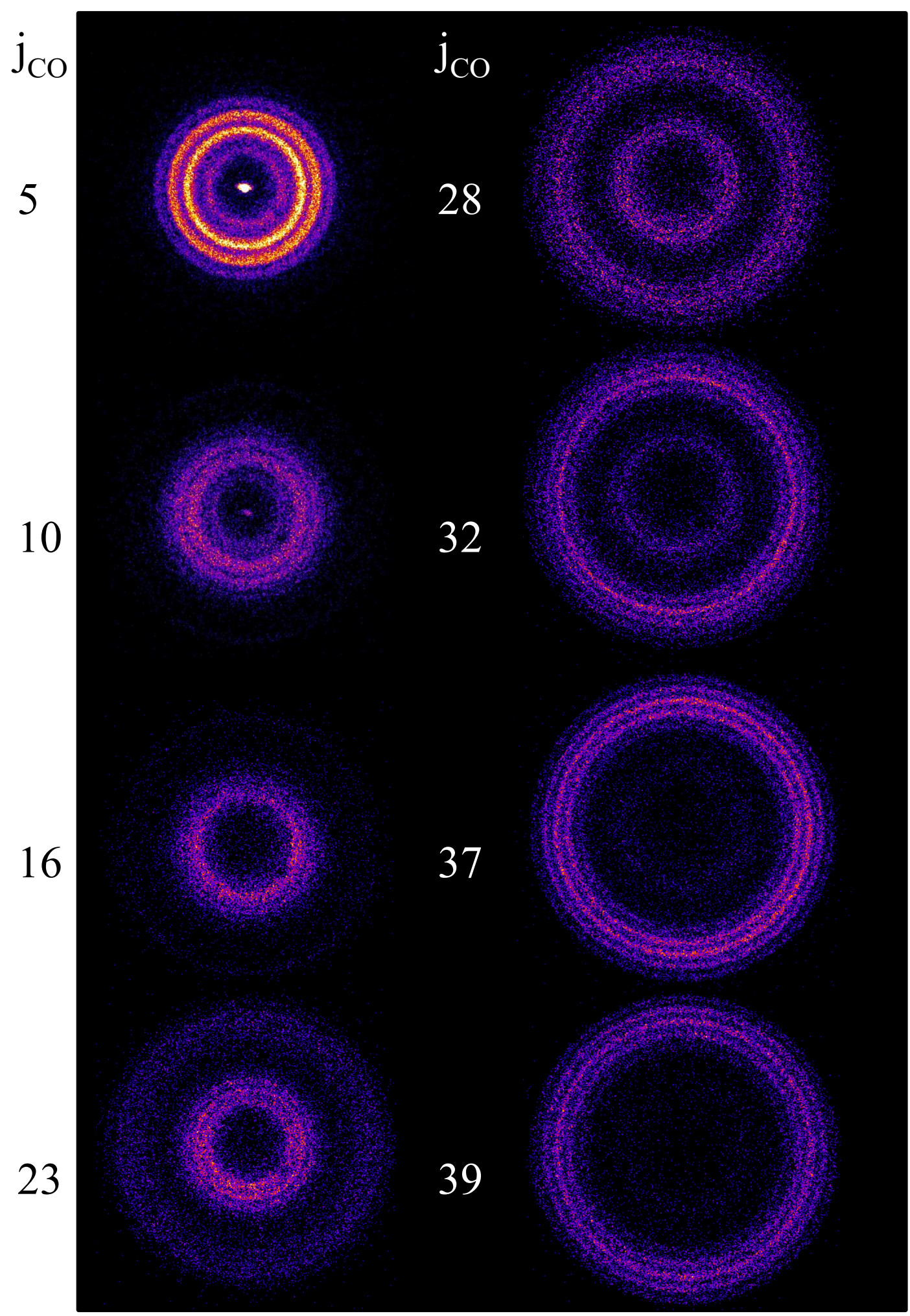


$2^{1} 4^{3}$

${ }^{\mathrm{p}} \mathrm{Q}_{1,0}(1) \rightarrow 1_{01}$

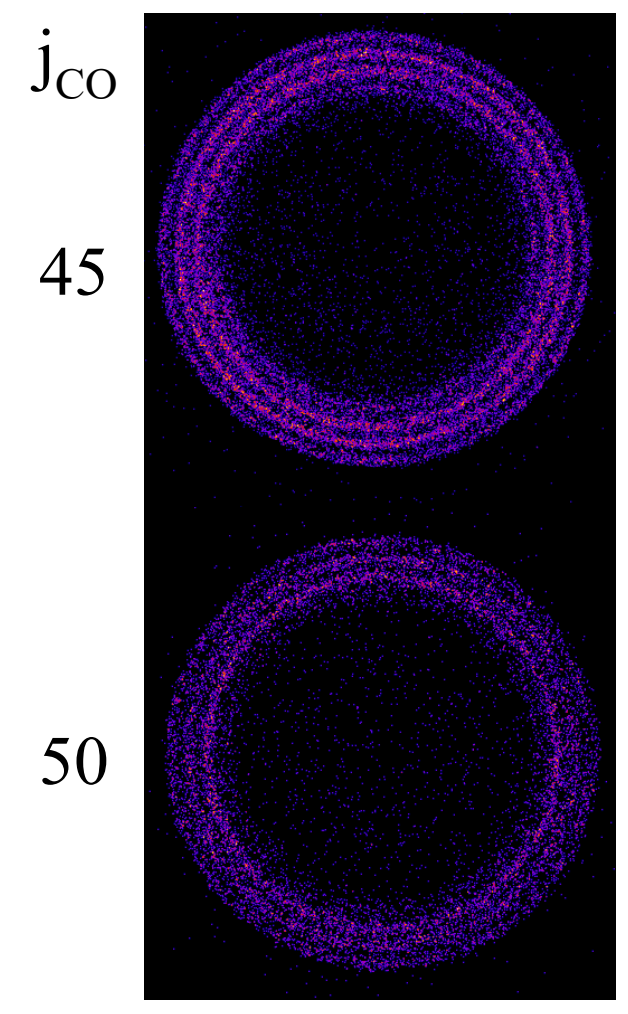


$2^{1} 4^{3}$

${ }^{\mathrm{p}} \mathrm{Q}_{1,1}(2) \rightarrow 2_{02}$

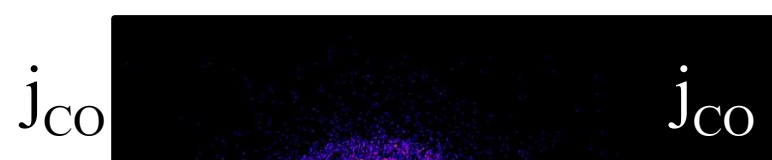

5

28

10

32

16

37

23

39 
$2^{1} 4^{3}$

${ }^{\mathrm{p}} \mathrm{Q}_{1,1}(2) \rightarrow 2_{02}$

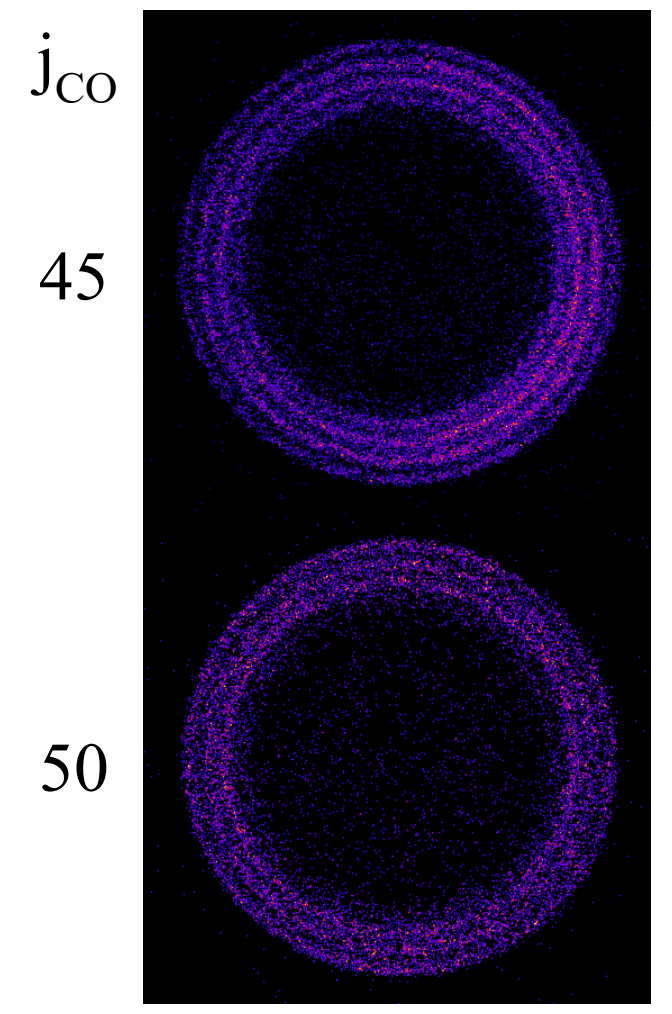


$2^{1} 4^{3}$

$$
{ }^{\mathrm{p}} \mathrm{R}_{1,2}(2) \rightarrow 3_{03}
$$

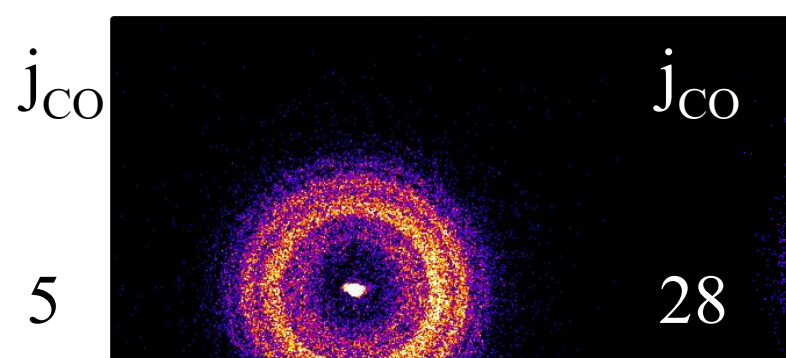

10

32

16

37

23

39 


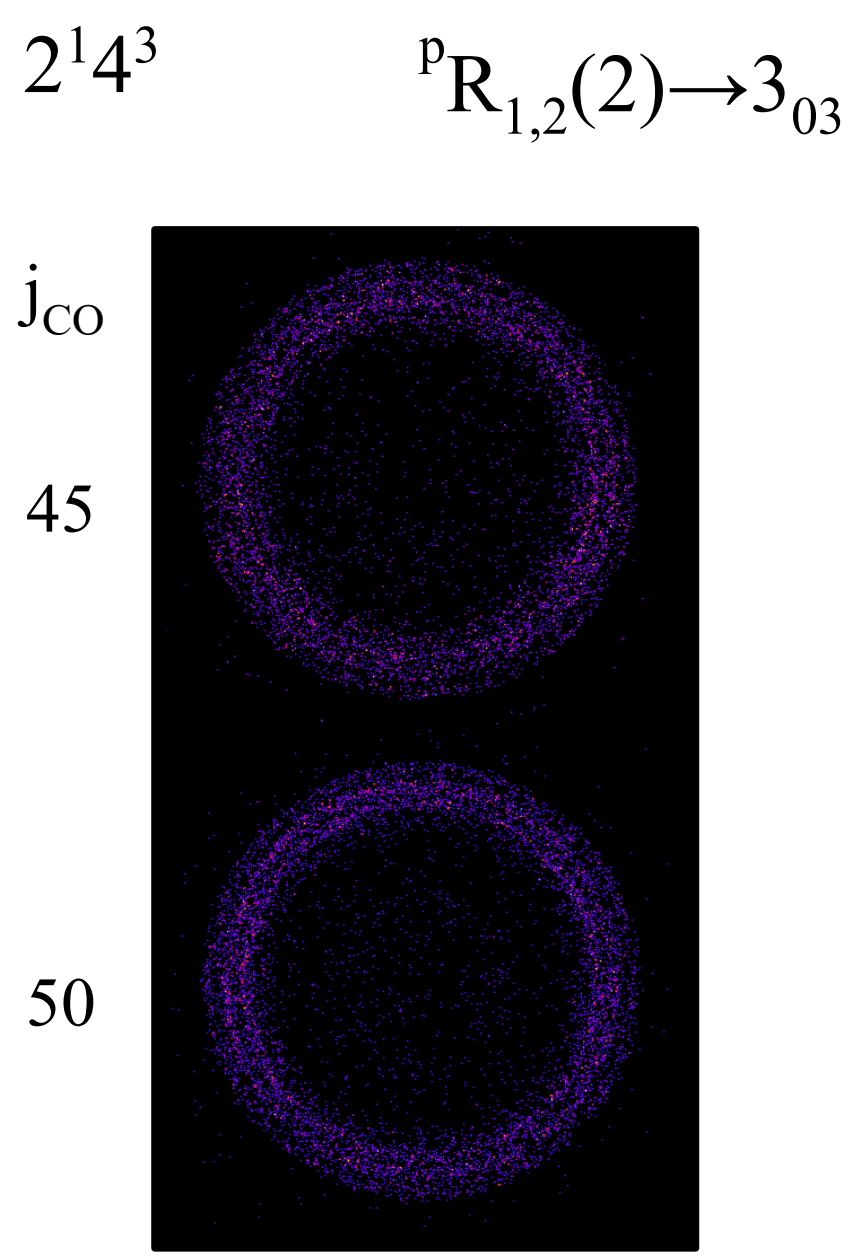


$2^{1} 4^{3}$

${ }^{\mathrm{p}} \mathrm{P}_{1,5}(5) \rightarrow 4_{04}$

$\mathrm{j}_{\mathrm{CO}}$
$\mathrm{j}_{\mathrm{CO}}$
10
45

16

23

28 
$2^{2} 4^{1}$

${ }^{\mathrm{r}} \mathrm{R}_{0,0}(0) \rightarrow 1_{11}$

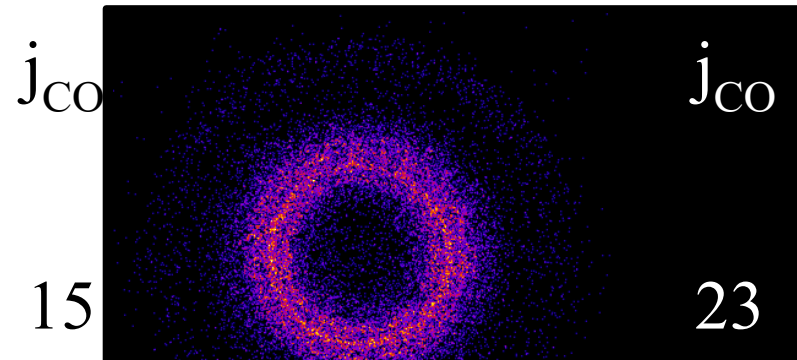

16

28

20

32

21

37 
$2^{2} 4^{1}$

${ }^{\mathrm{r}} \mathrm{R}_{0,0}(0) \rightarrow 1_{11}$

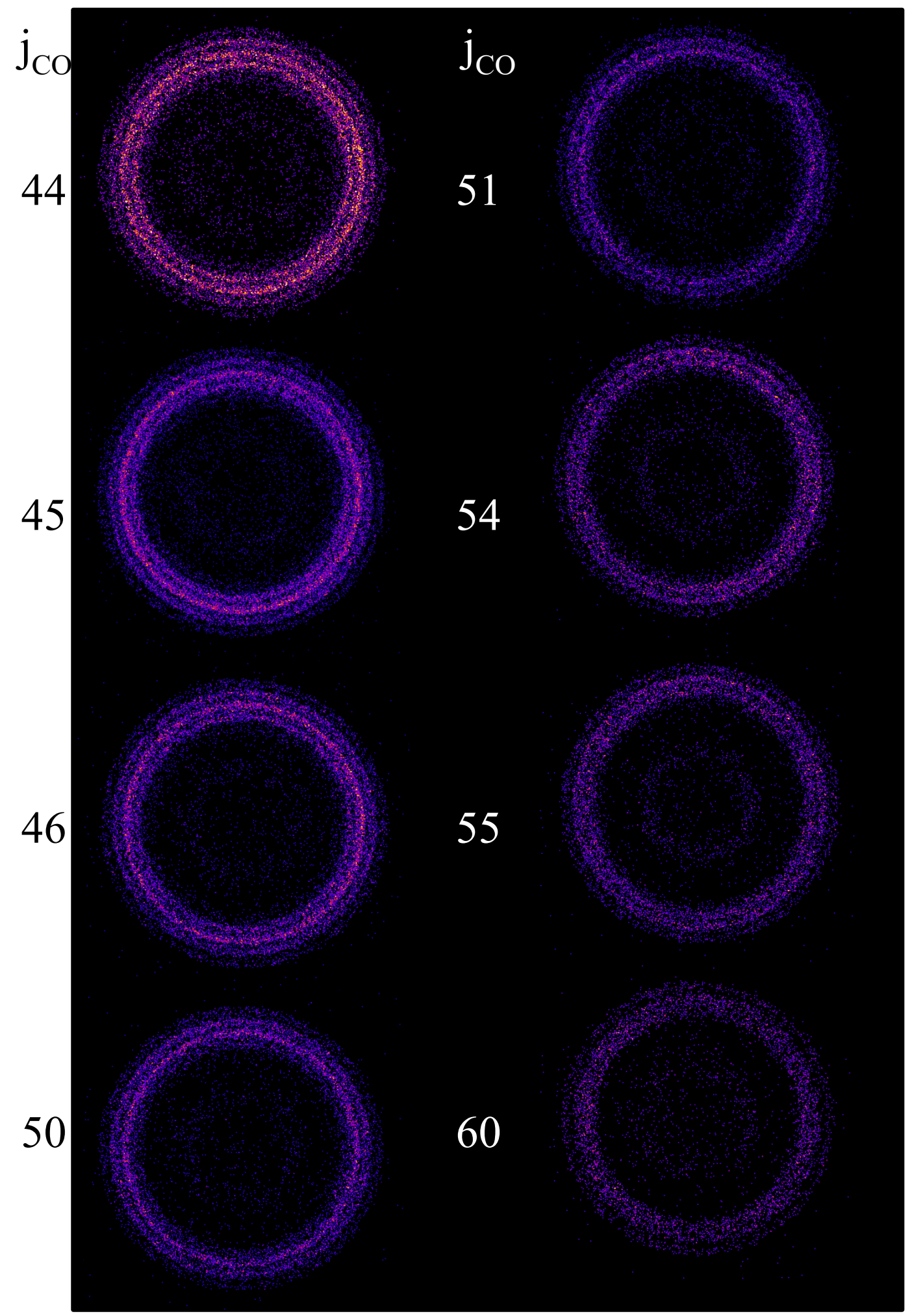


$2^{2} 4^{1}$

${ }^{\mathrm{r}} \mathrm{Q}_{0,1}(1) \rightarrow 1_{10}$

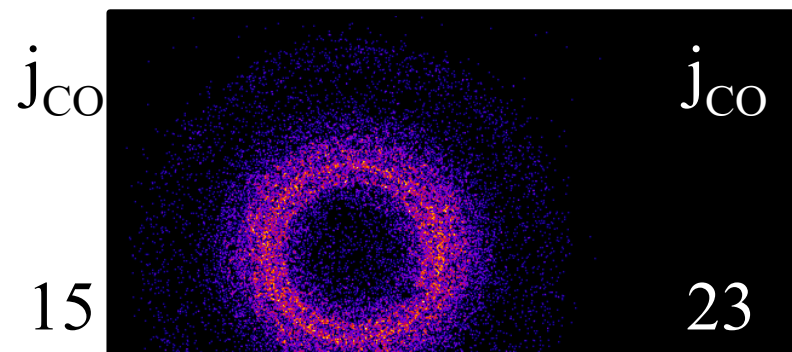

16

28

20

32

21

37 
$2^{2} 4^{1}$

${ }^{\mathrm{r}} \mathrm{Q}_{0,1}(1) \rightarrow 1_{10}$

$\begin{array}{cc}\mathrm{j}_{\mathrm{CO}} & \mathrm{j}_{\mathrm{CO}} \\ 44 & 51\end{array}$

45

54

46

55

50

60 
$2^{2} 4^{1} \quad{ }^{\mathrm{r}} \mathrm{Q}_{0,2}(2) \rightarrow 2_{11}$

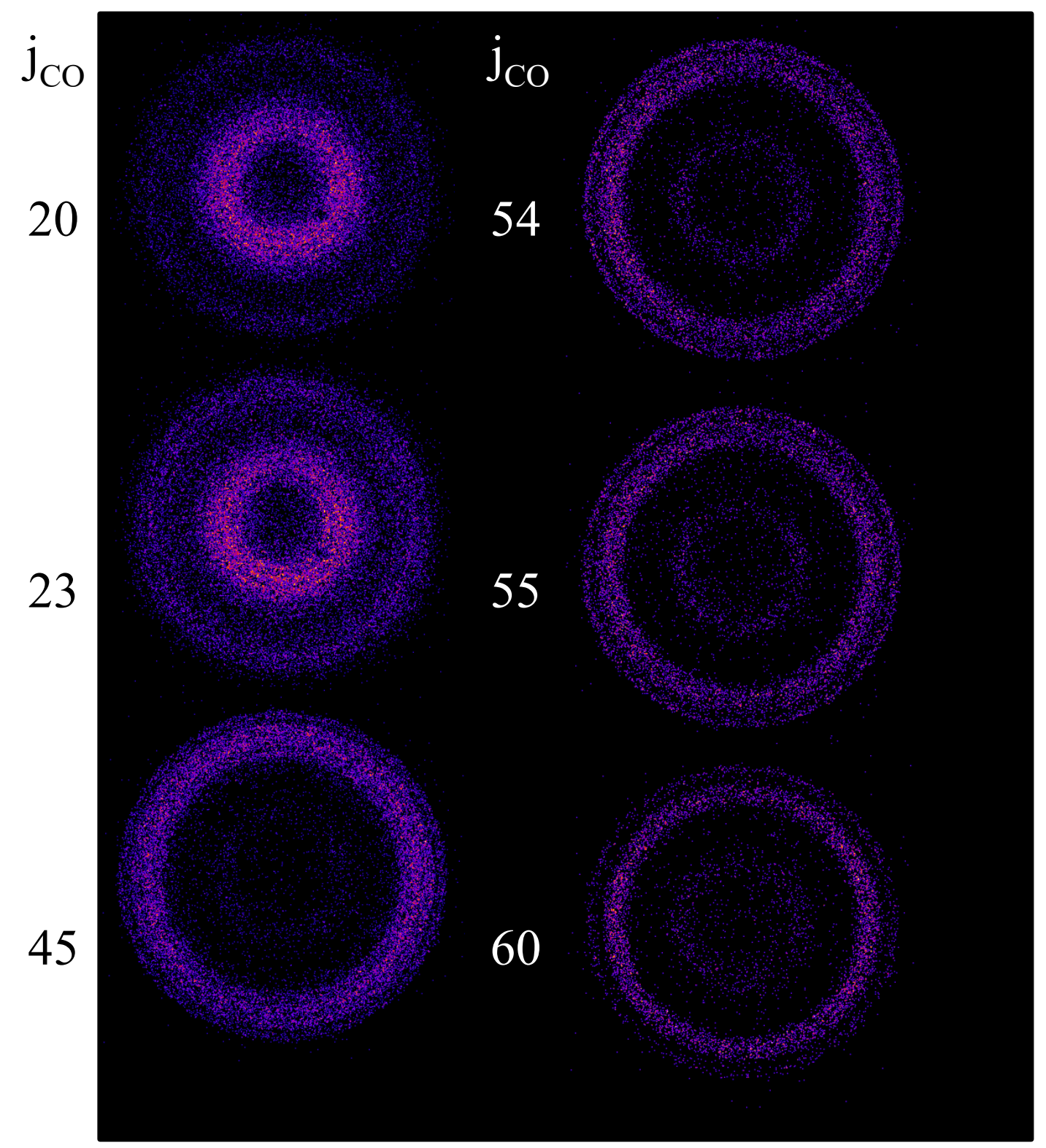


$2^{2} 4^{1} \quad{ }^{\mathrm{r}} \mathrm{R}_{0,1}(1) \rightarrow 2_{12}$

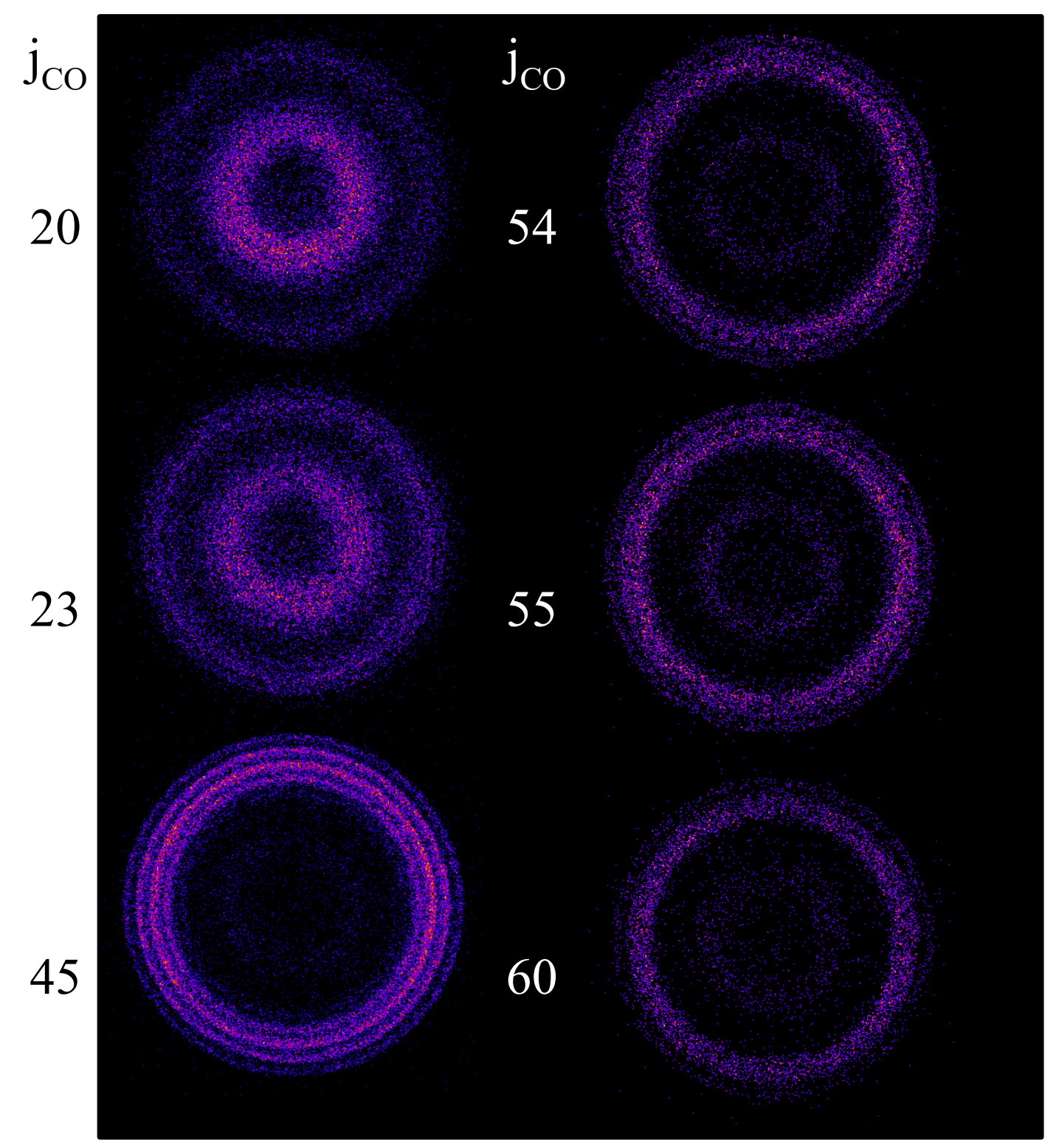


$2^{2} 4^{3}$

${ }^{\mathrm{r}} \mathrm{R}_{0,0}(0) \rightarrow 1_{11}$

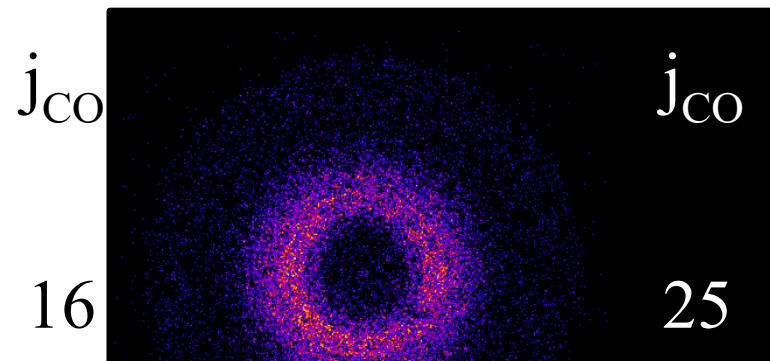

20

28

22

32

23

37 
$2^{2} 4^{3}$

${ }^{\mathrm{r}} \mathrm{R}_{0,0}(0) \rightarrow 1_{11}$

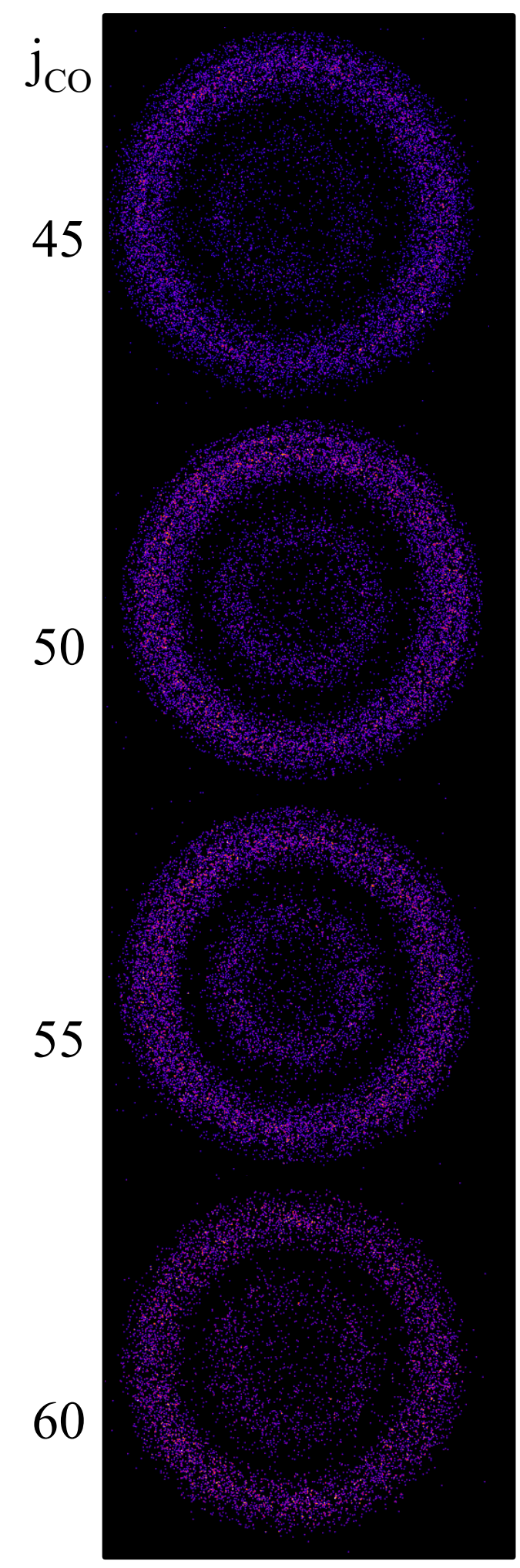


$2^{2} 4^{3}$

${ }^{\mathrm{r}} \mathrm{Q}_{0,1}(1) \rightarrow 1_{10}$

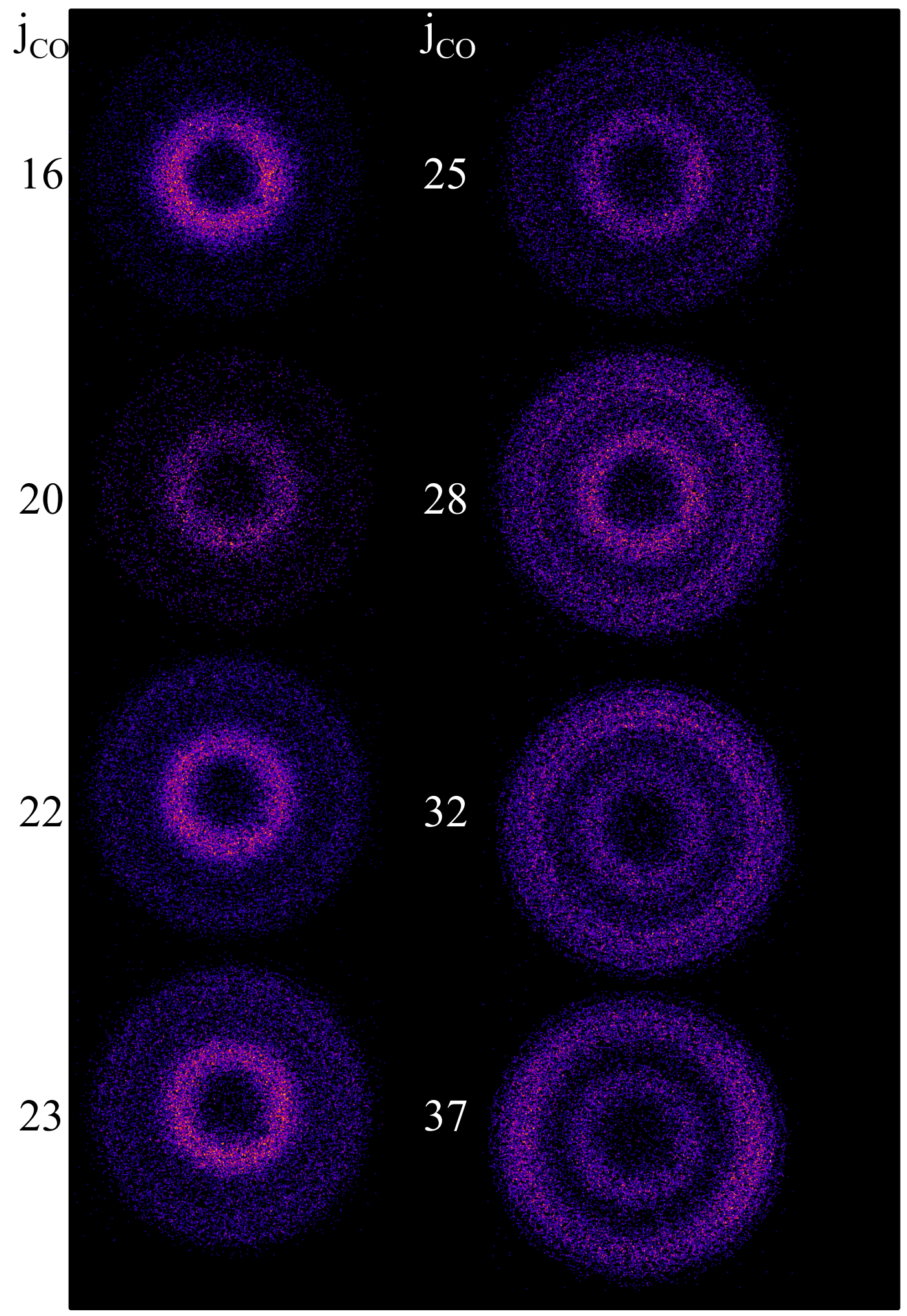


$2^{2} 4^{3}$

${ }^{\mathrm{r}} \mathrm{Q}_{0,1}(1) \rightarrow 1_{10}$

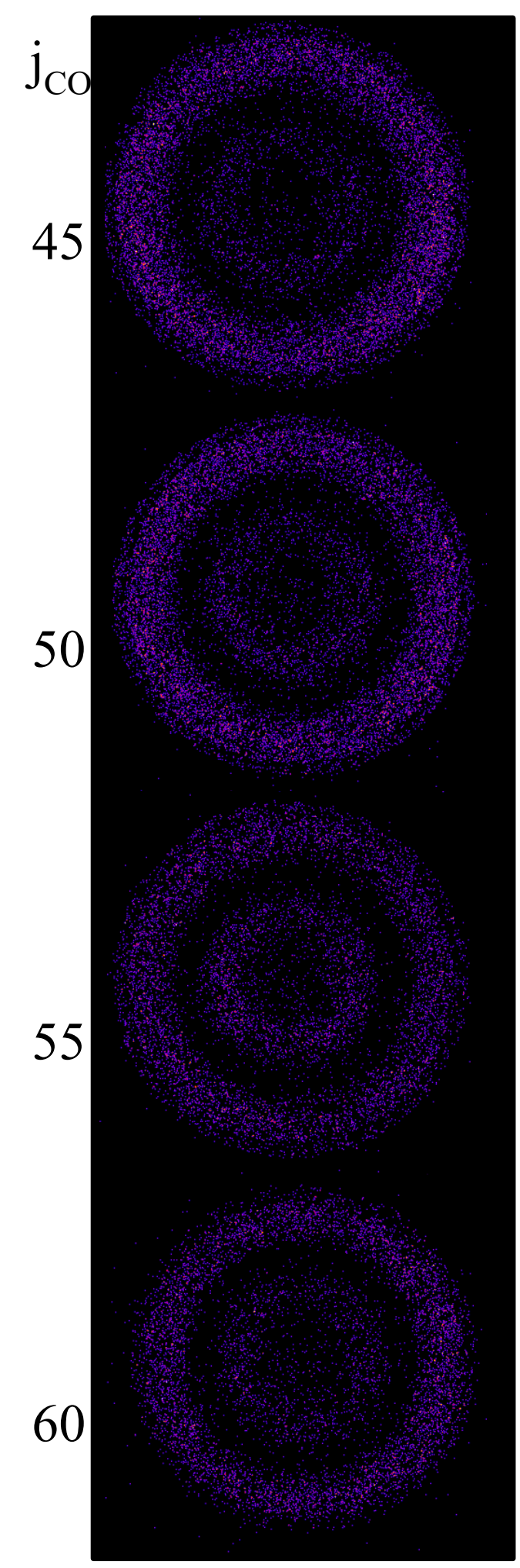


$2^{2} 4^{3}$

${ }^{\mathrm{r}} \mathrm{Q}_{0,2}(2) \rightarrow 2_{11}$

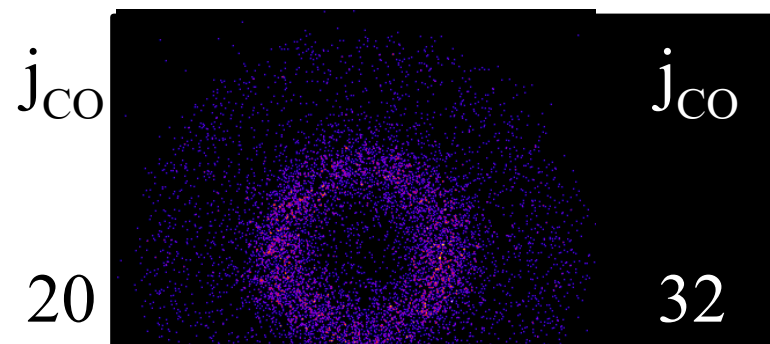

23

37

25

45

28 
$2^{2} 4^{3} \quad{ }^{\mathrm{r}} \mathrm{R}_{0,1}(1) \rightarrow 2_{12}$

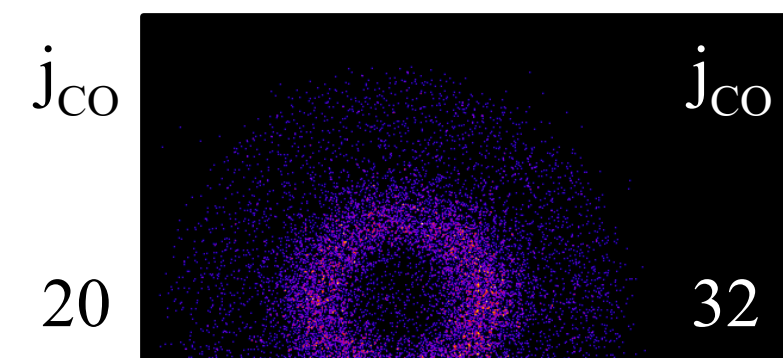

23

37

25

45

28 


\section{APPENDIX D}

Translational energy distributions of jco DC slice images for the different transitions used to exited formaldehyde are included here, with the rotational line indicated at the top of the page. 

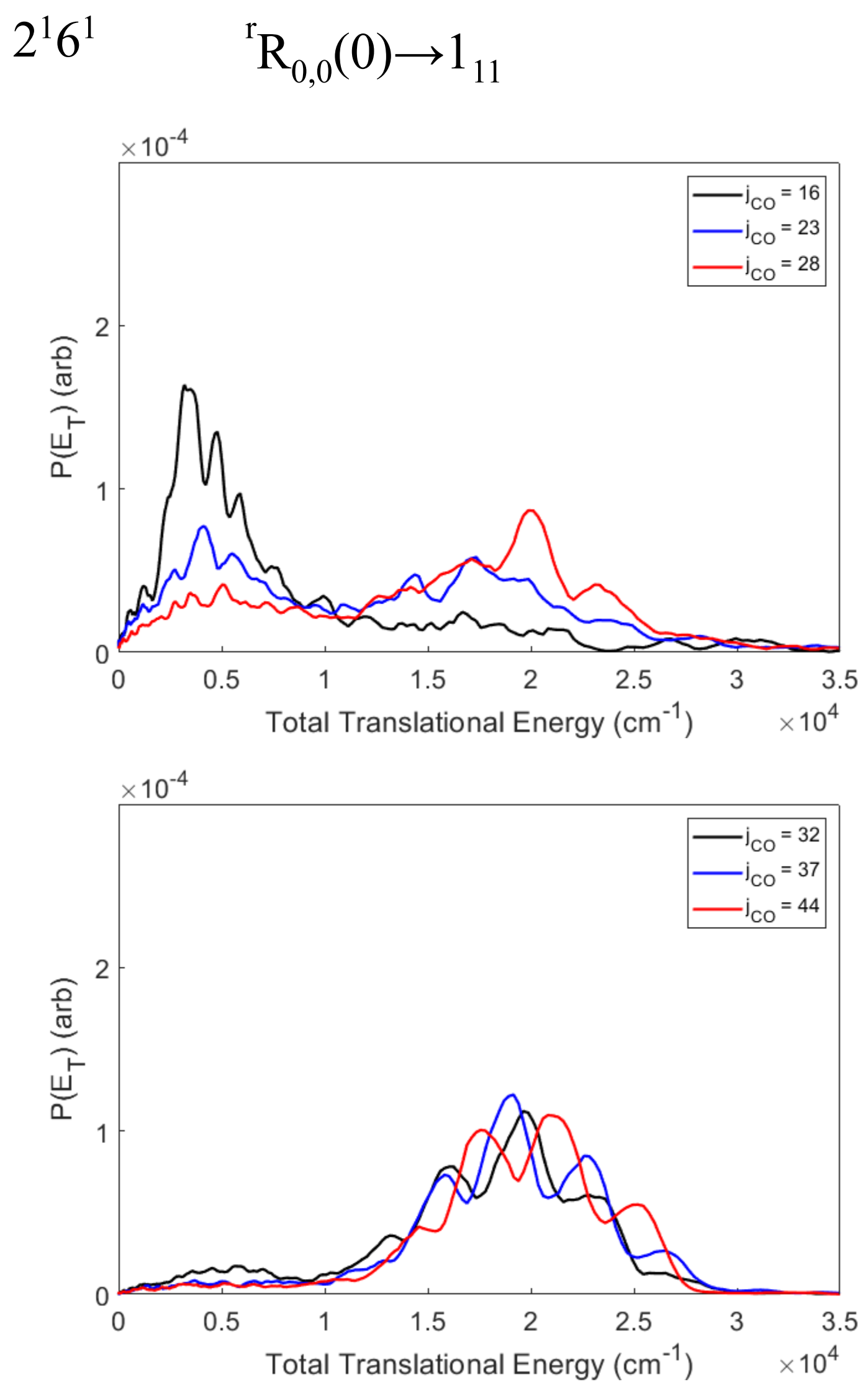


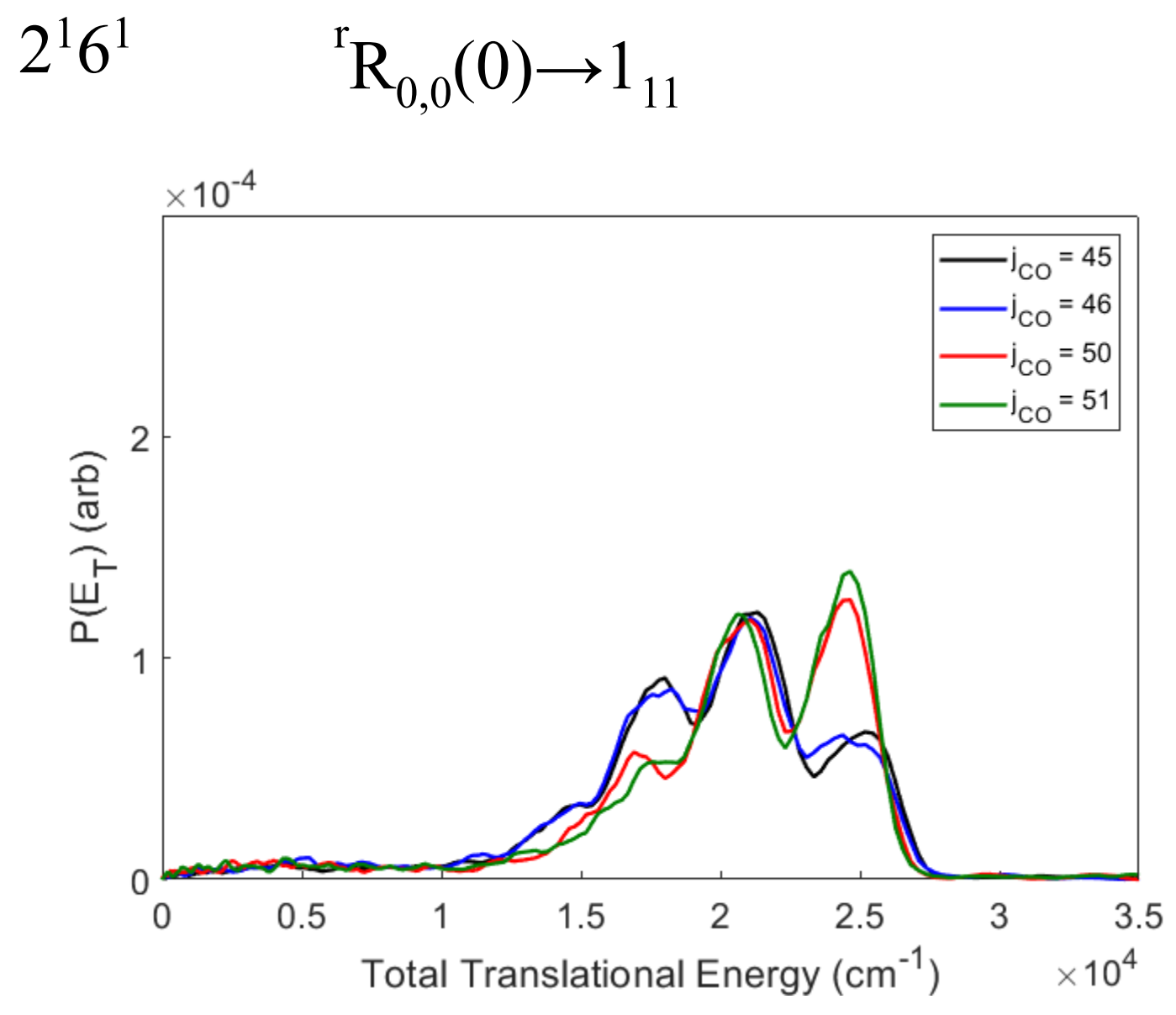



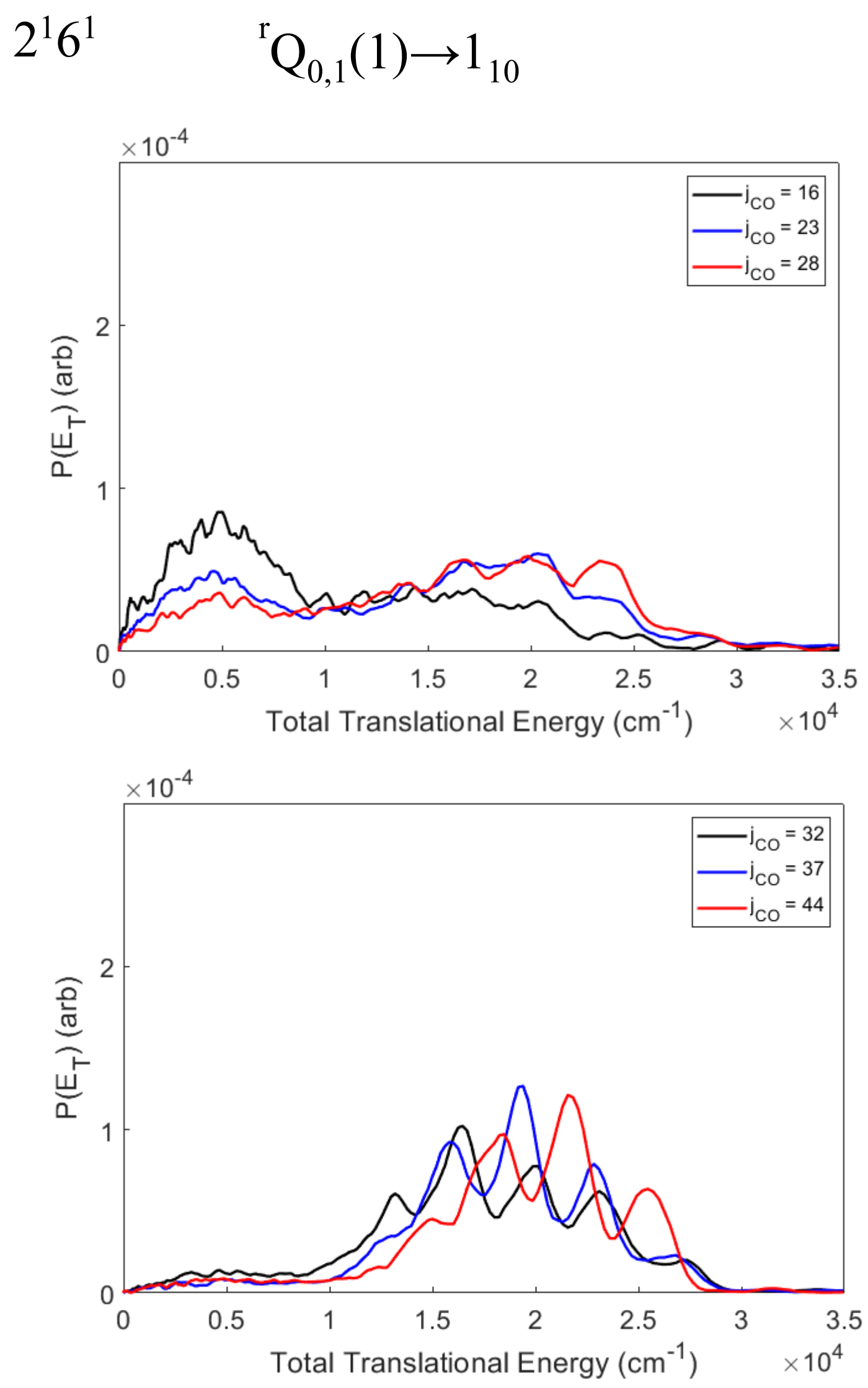


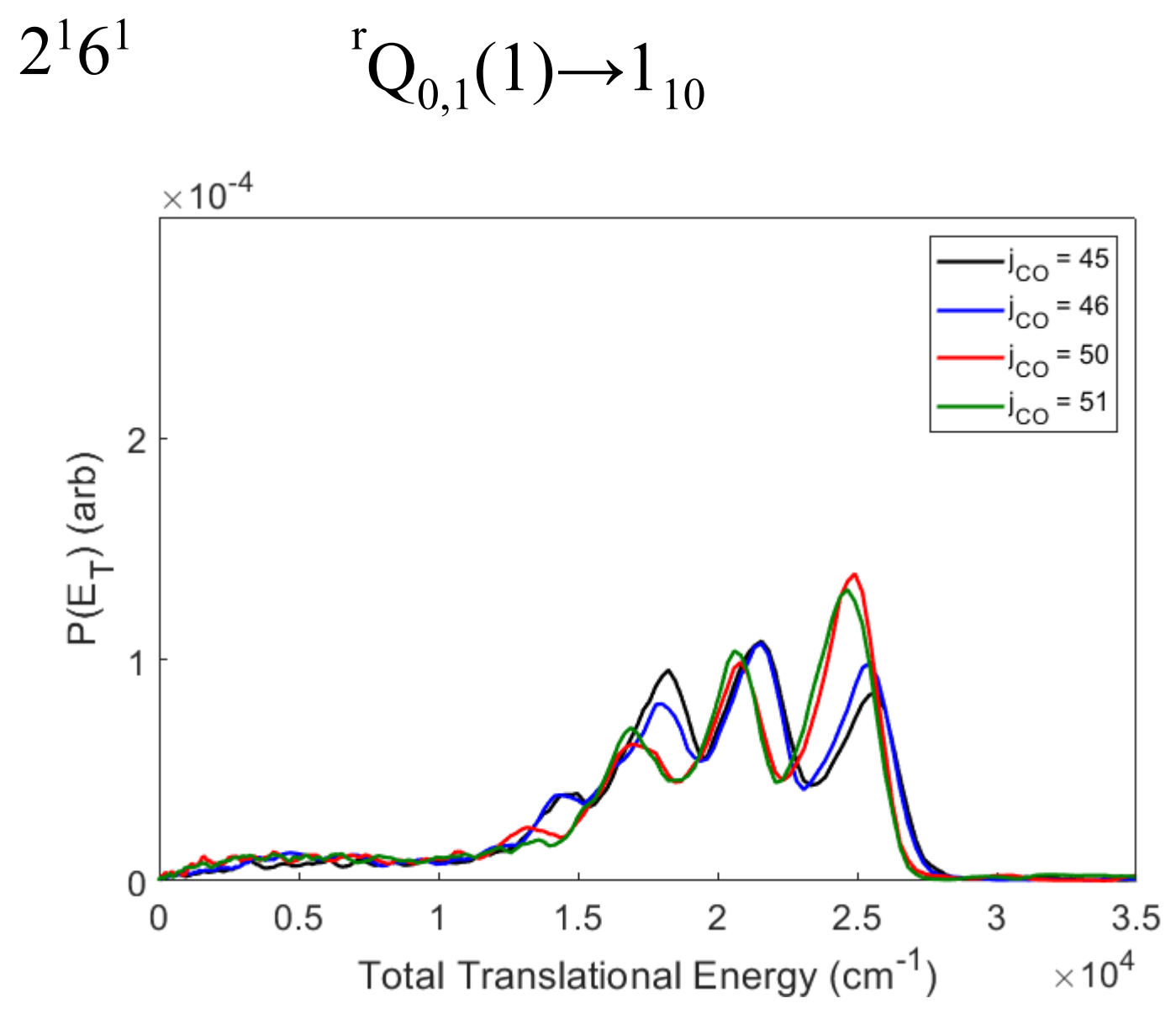



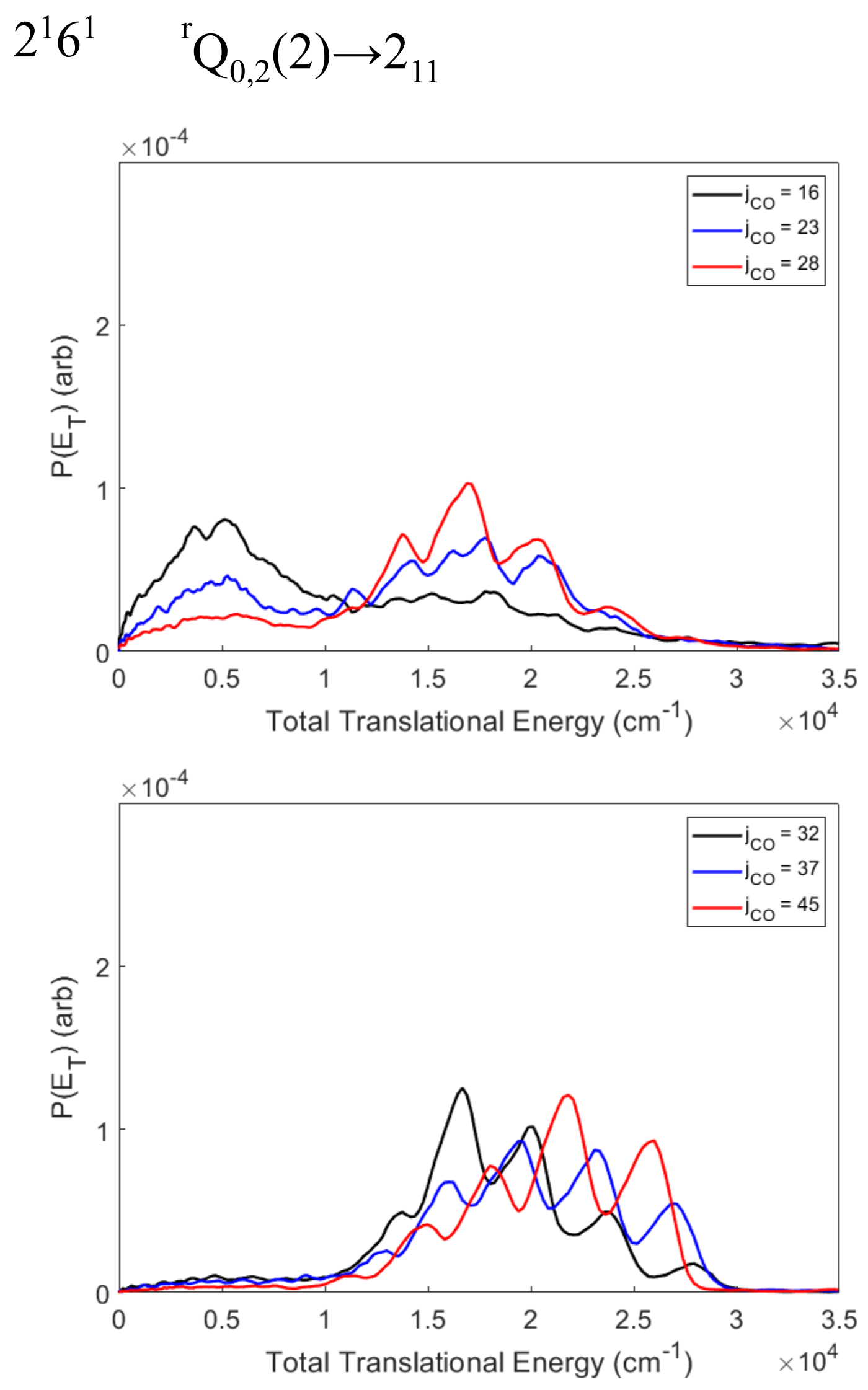

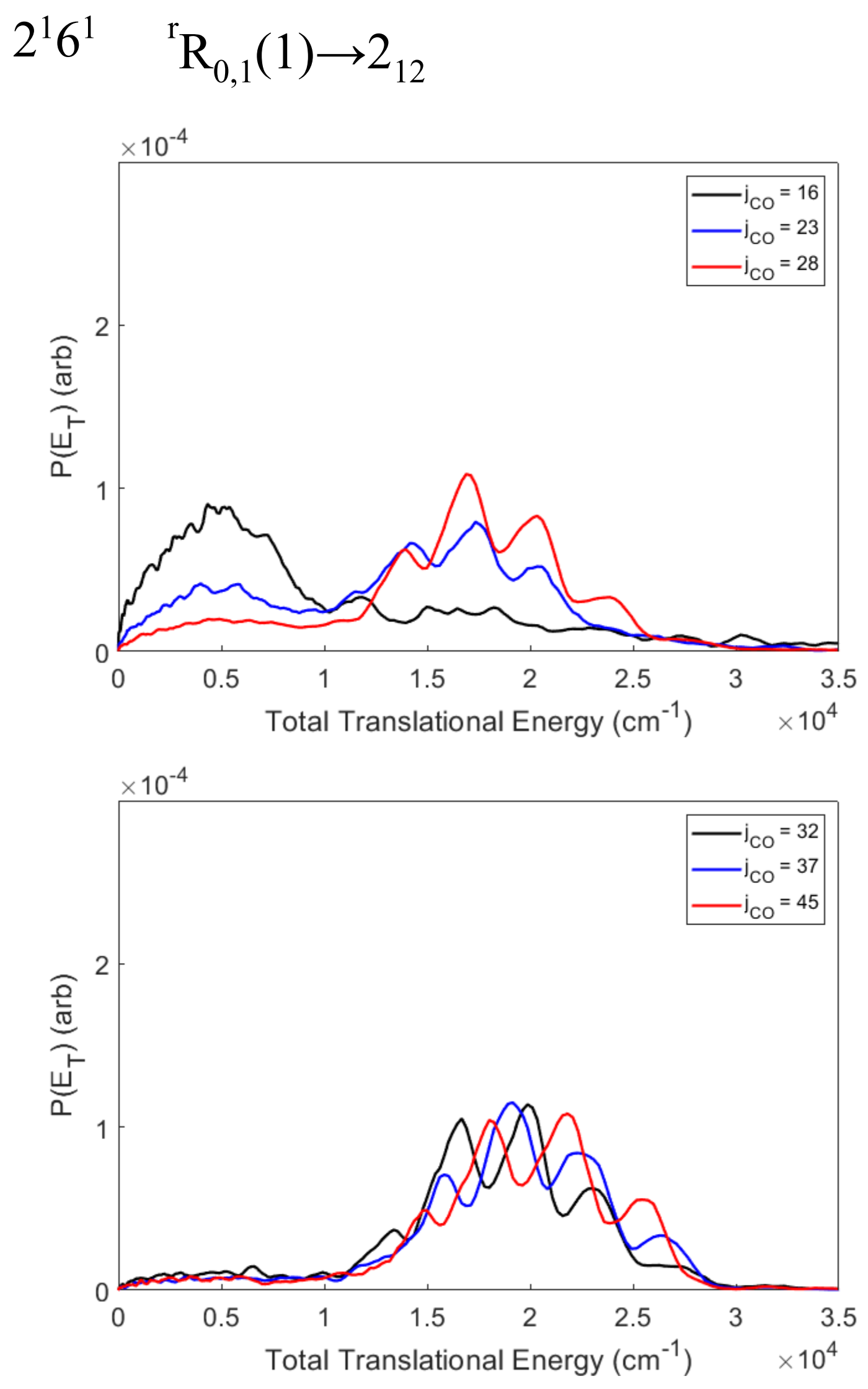

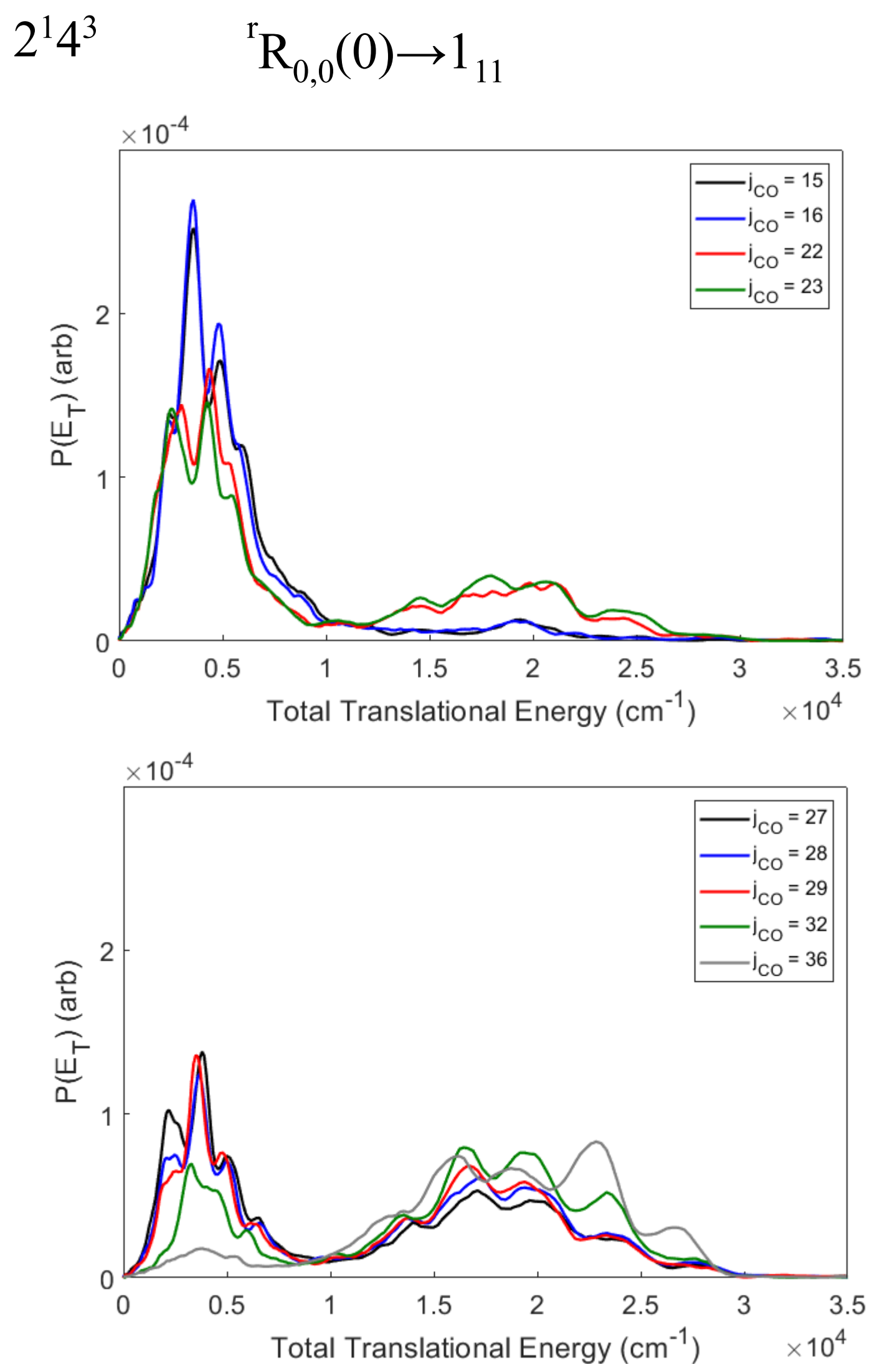

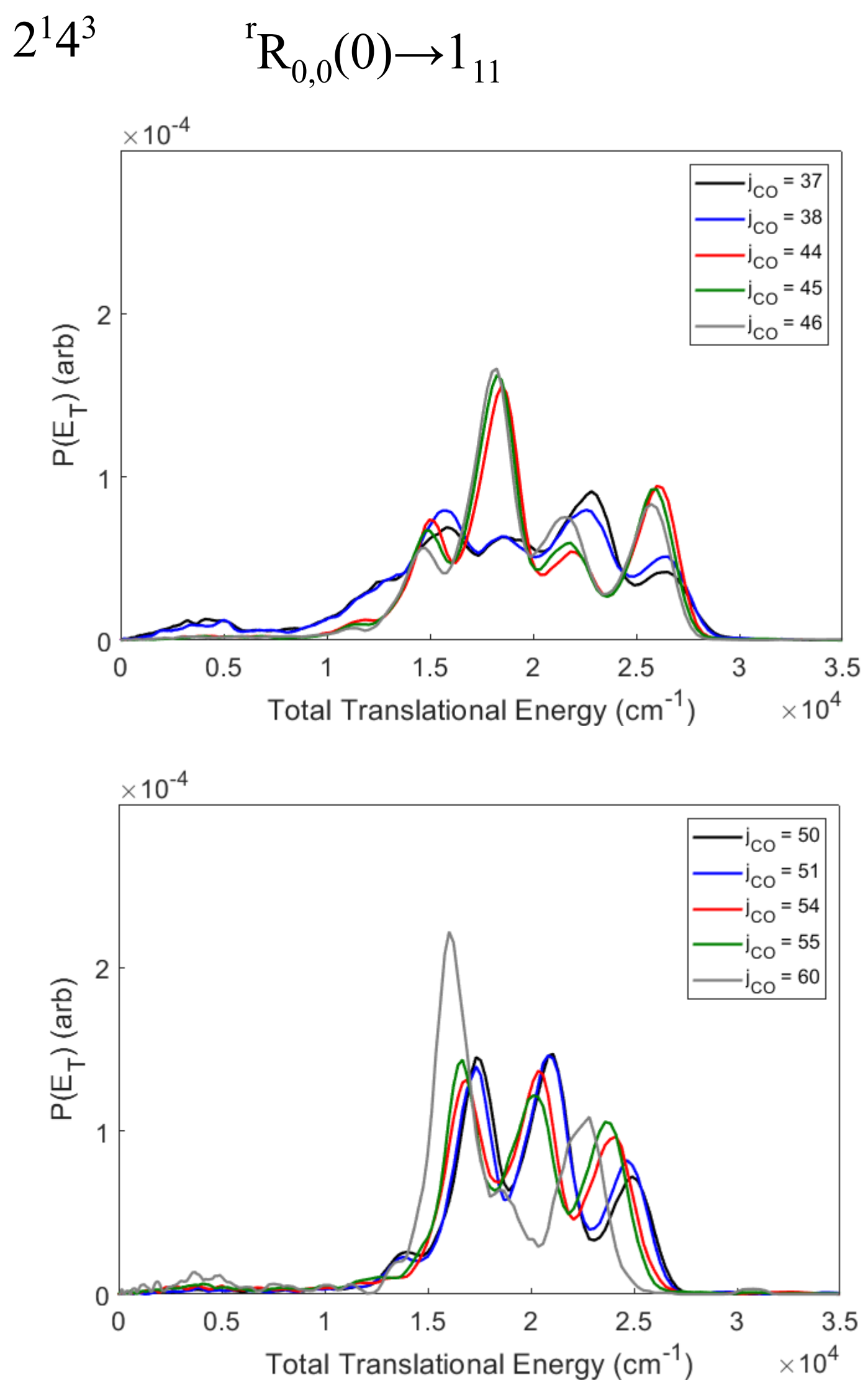

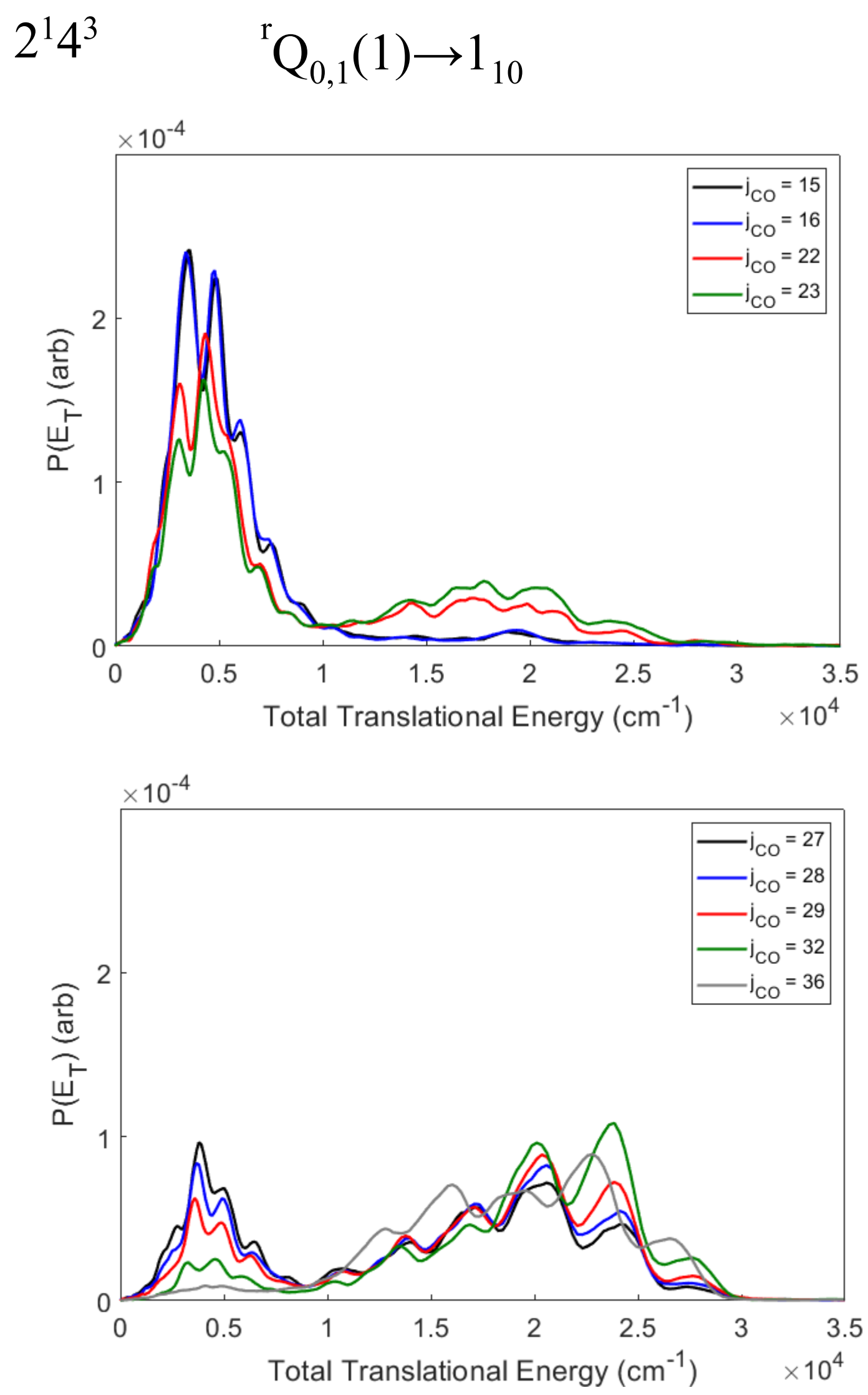

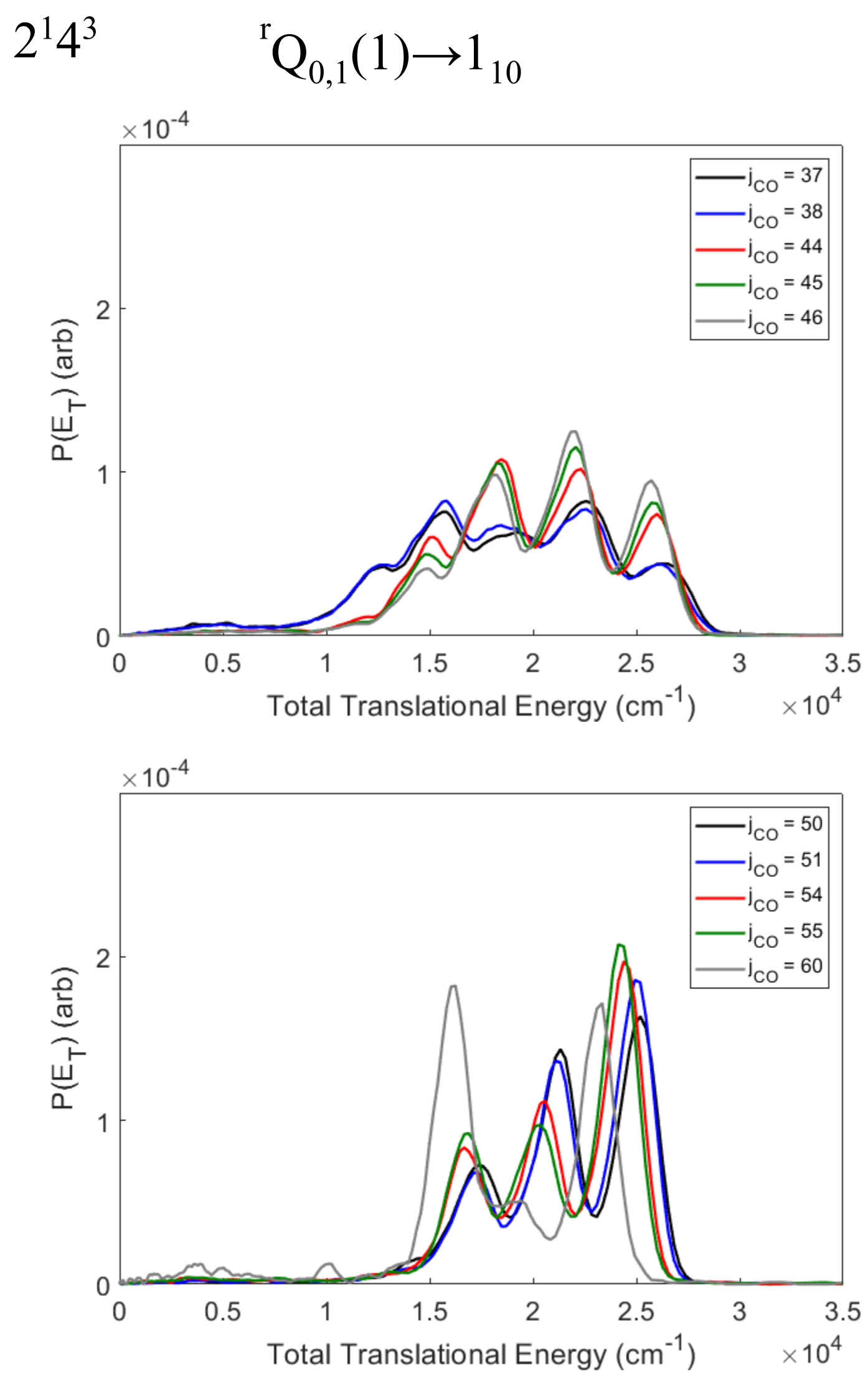

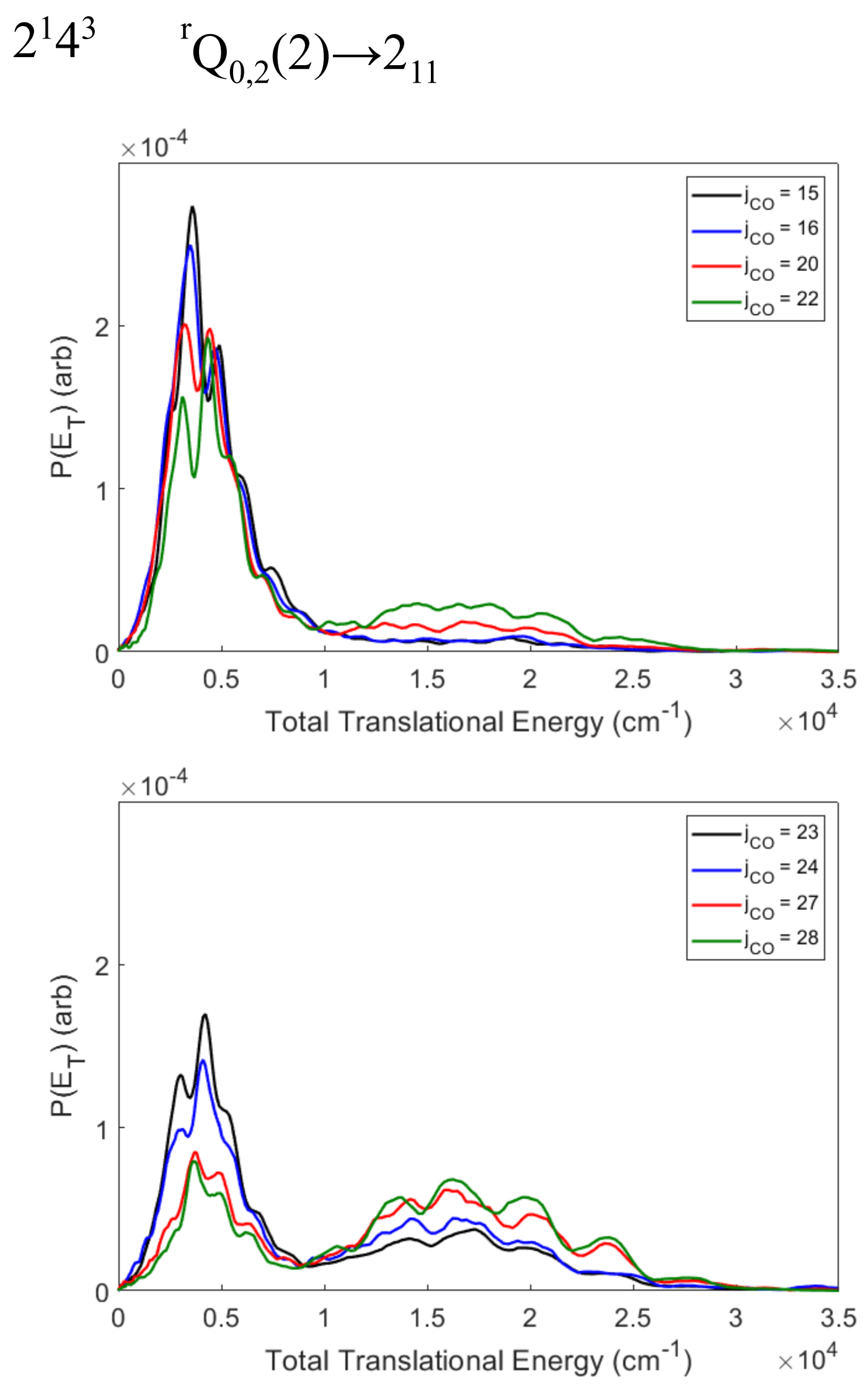

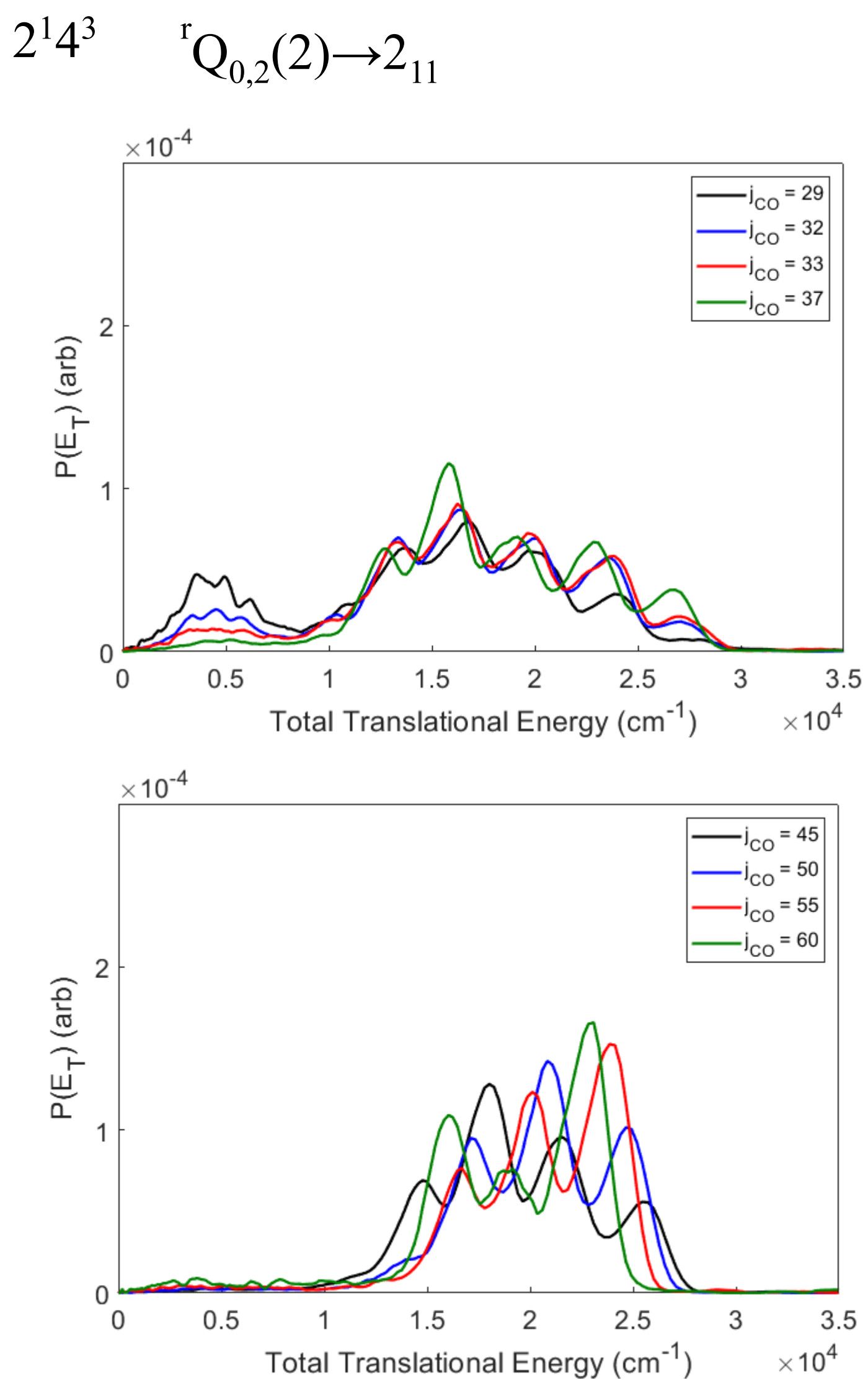


$$
2^{1} 4^{3} \quad{ }^{\mathrm{r}} \mathrm{R}_{0,1}(1) \rightarrow 2_{12}
$$
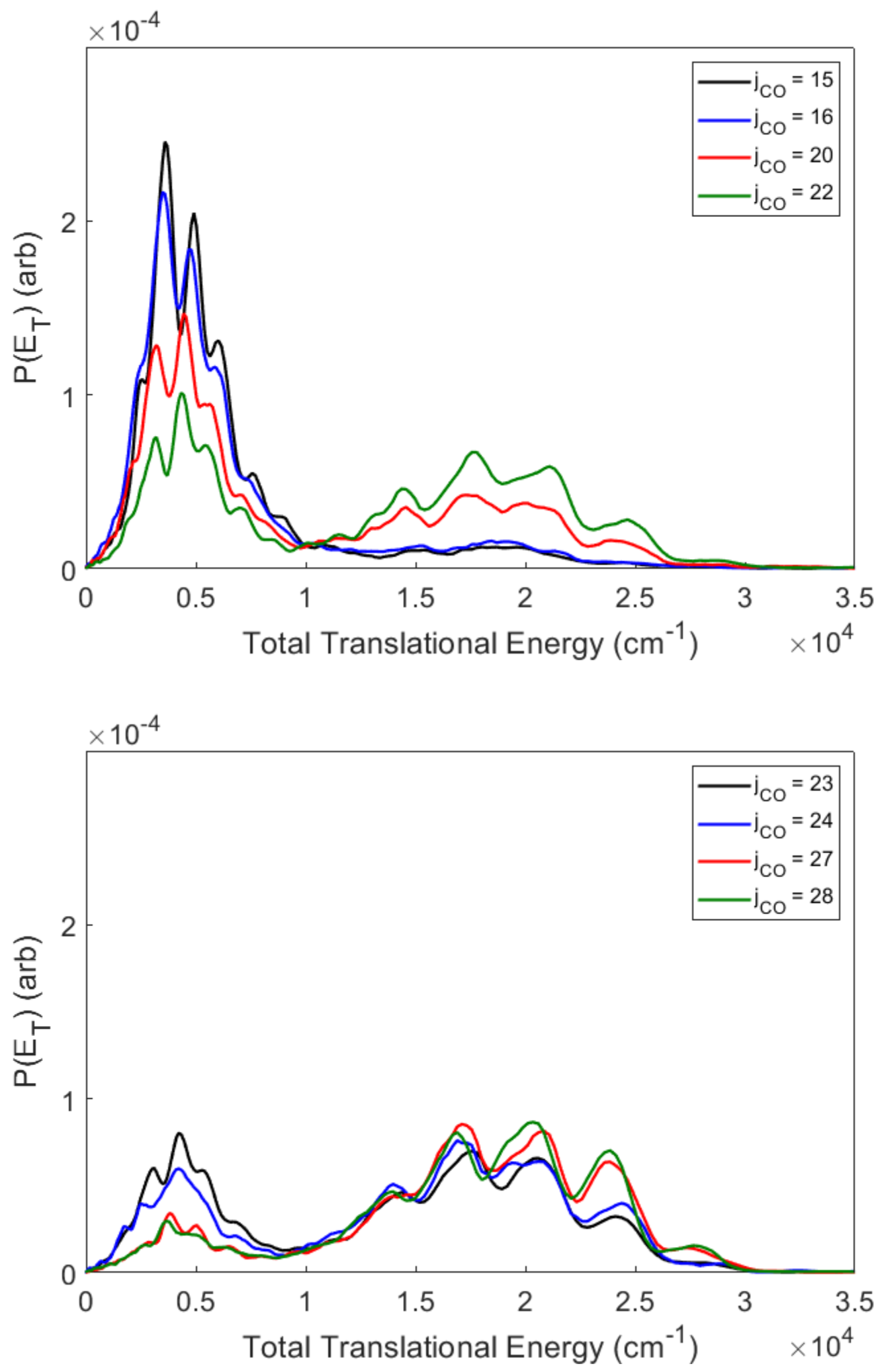

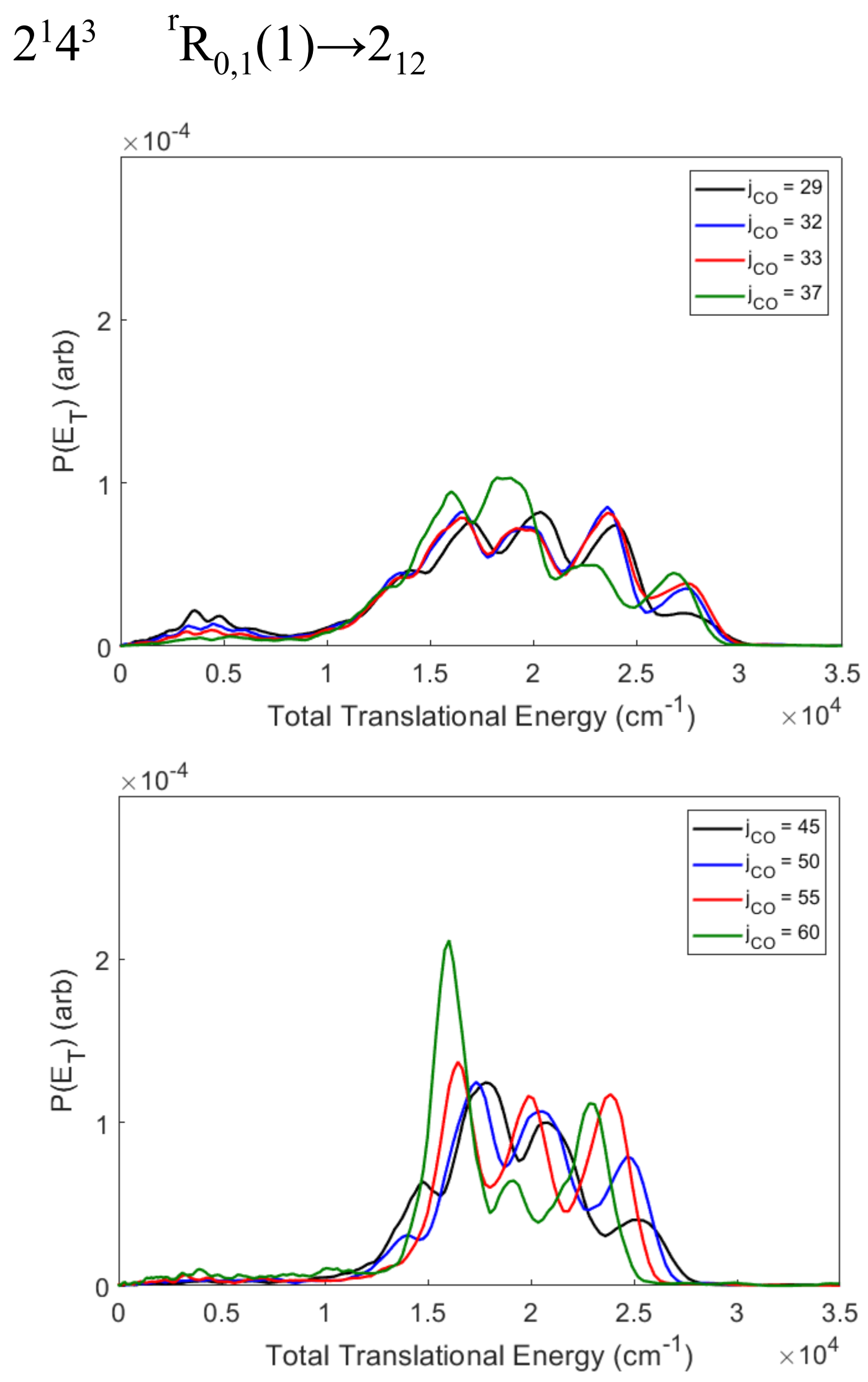

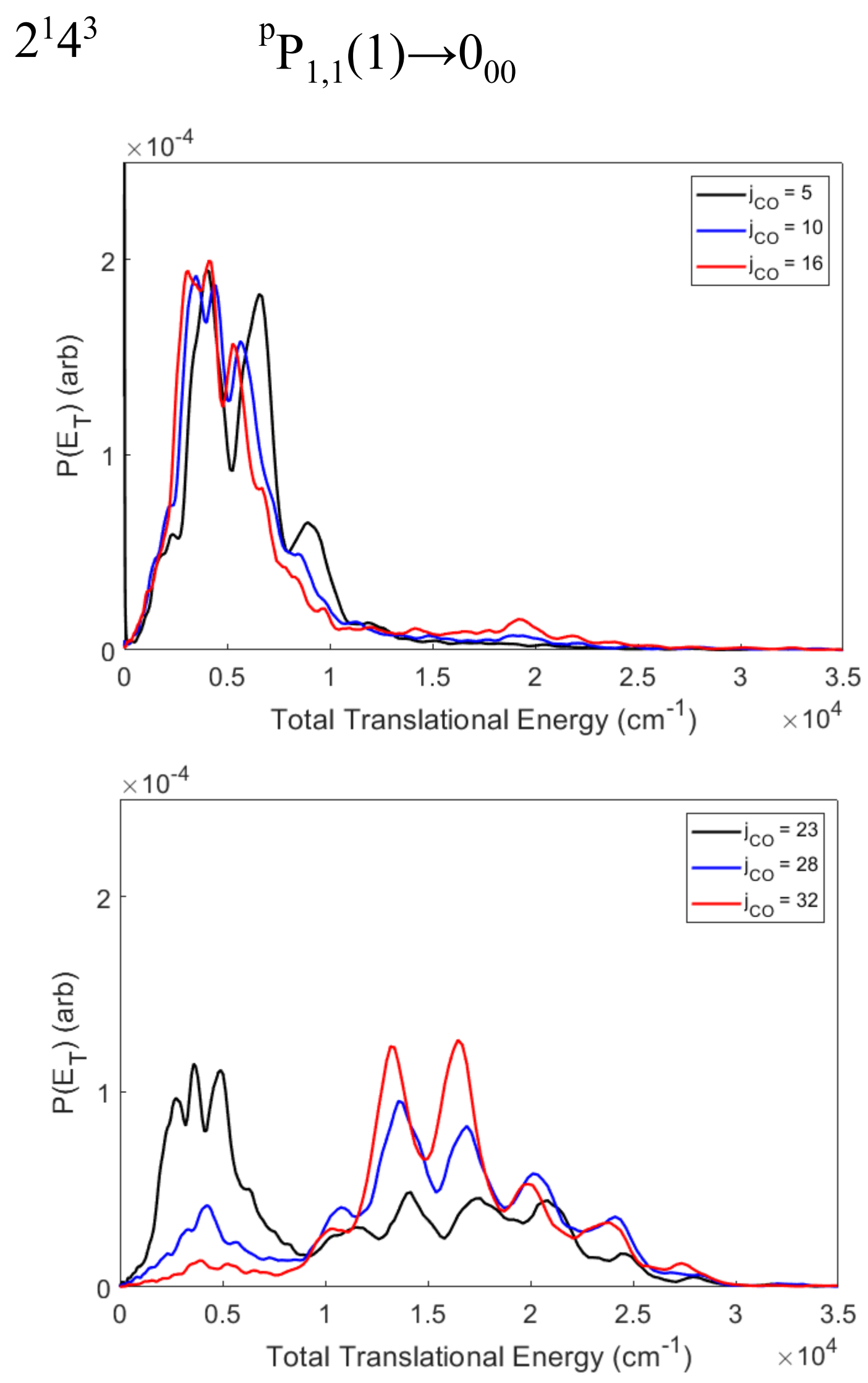


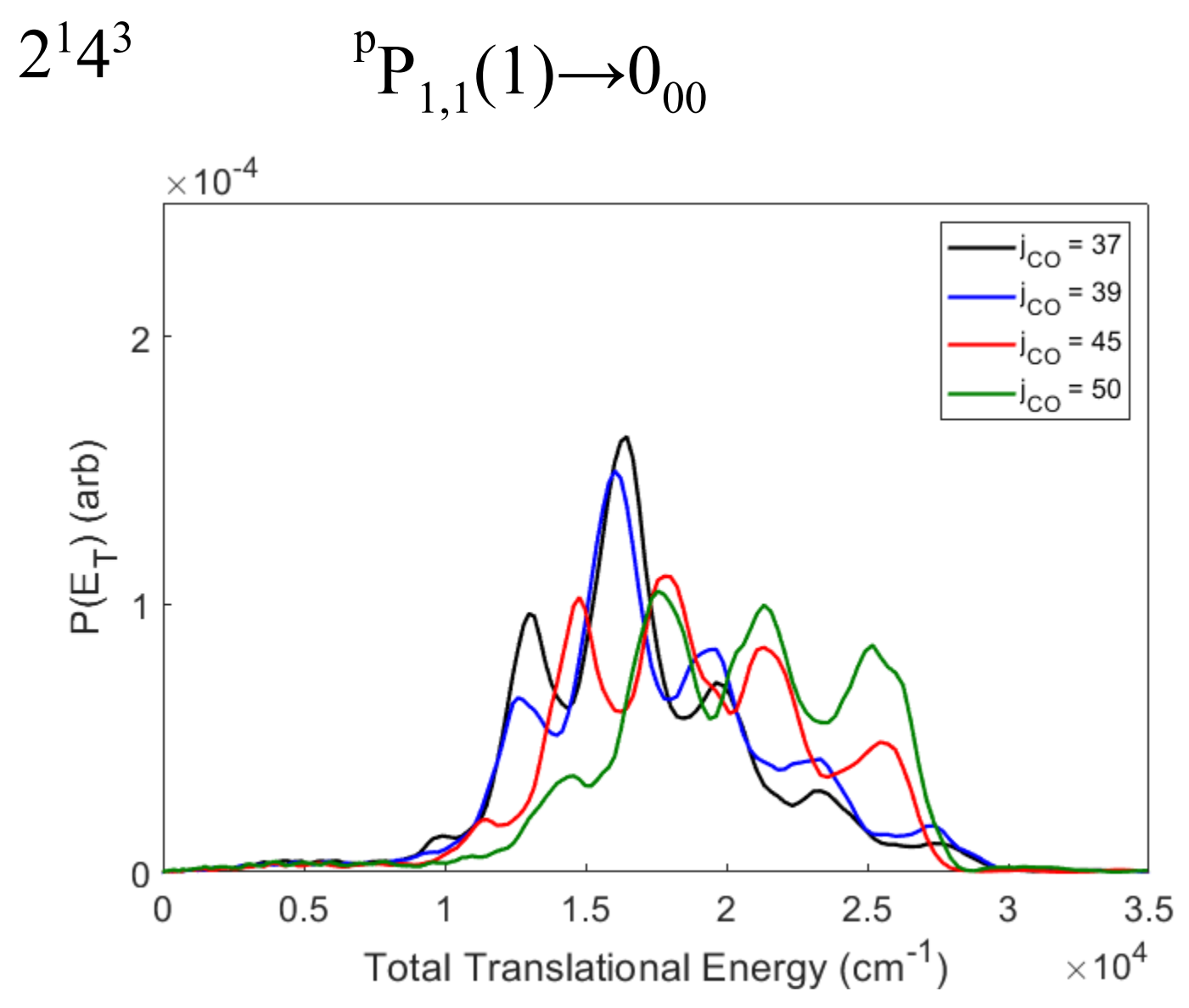



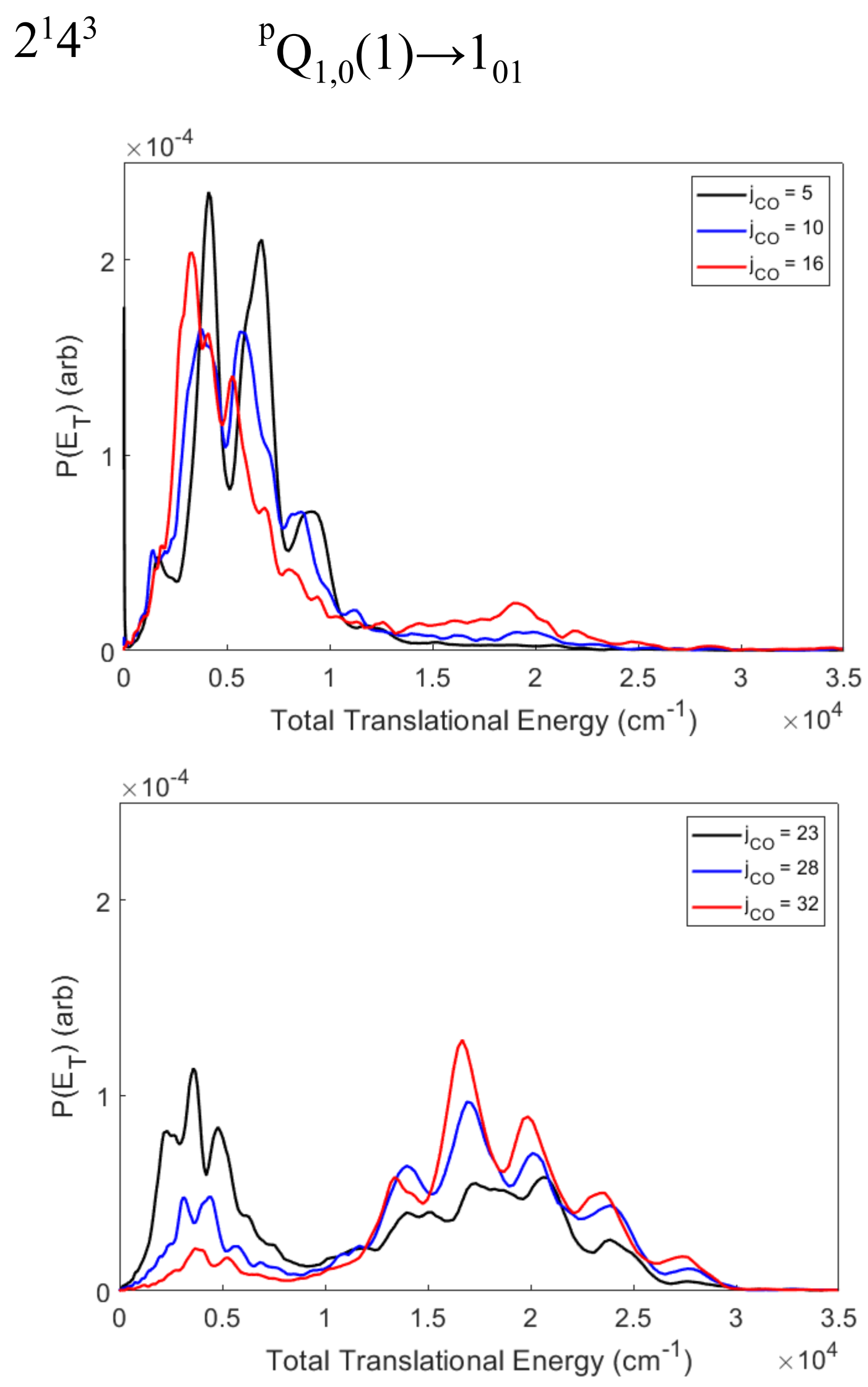
$2^{1} 4^{3}$

${ }^{\mathrm{p}} \mathrm{Q}_{1,0}(1) \rightarrow 1_{01}$

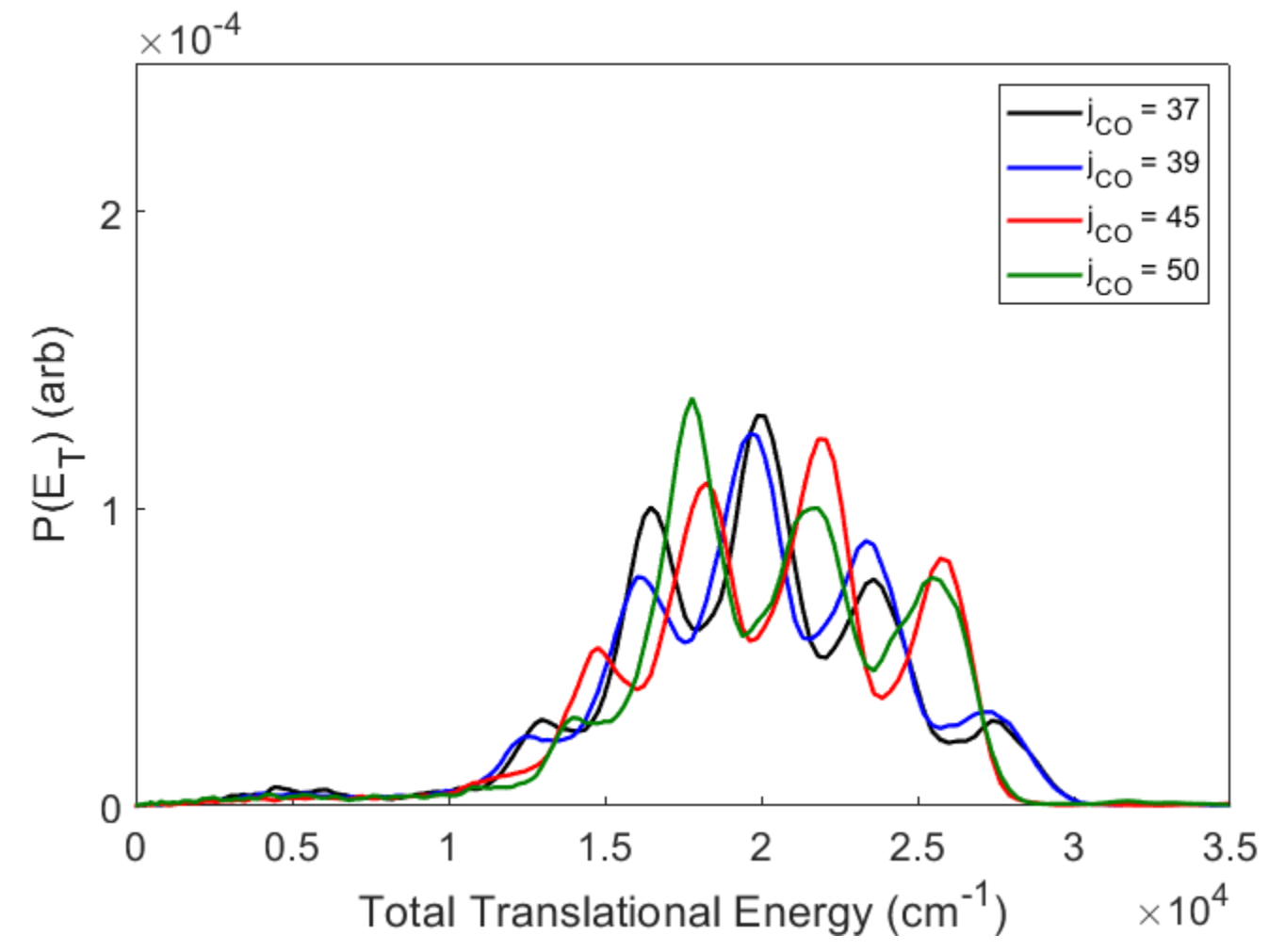



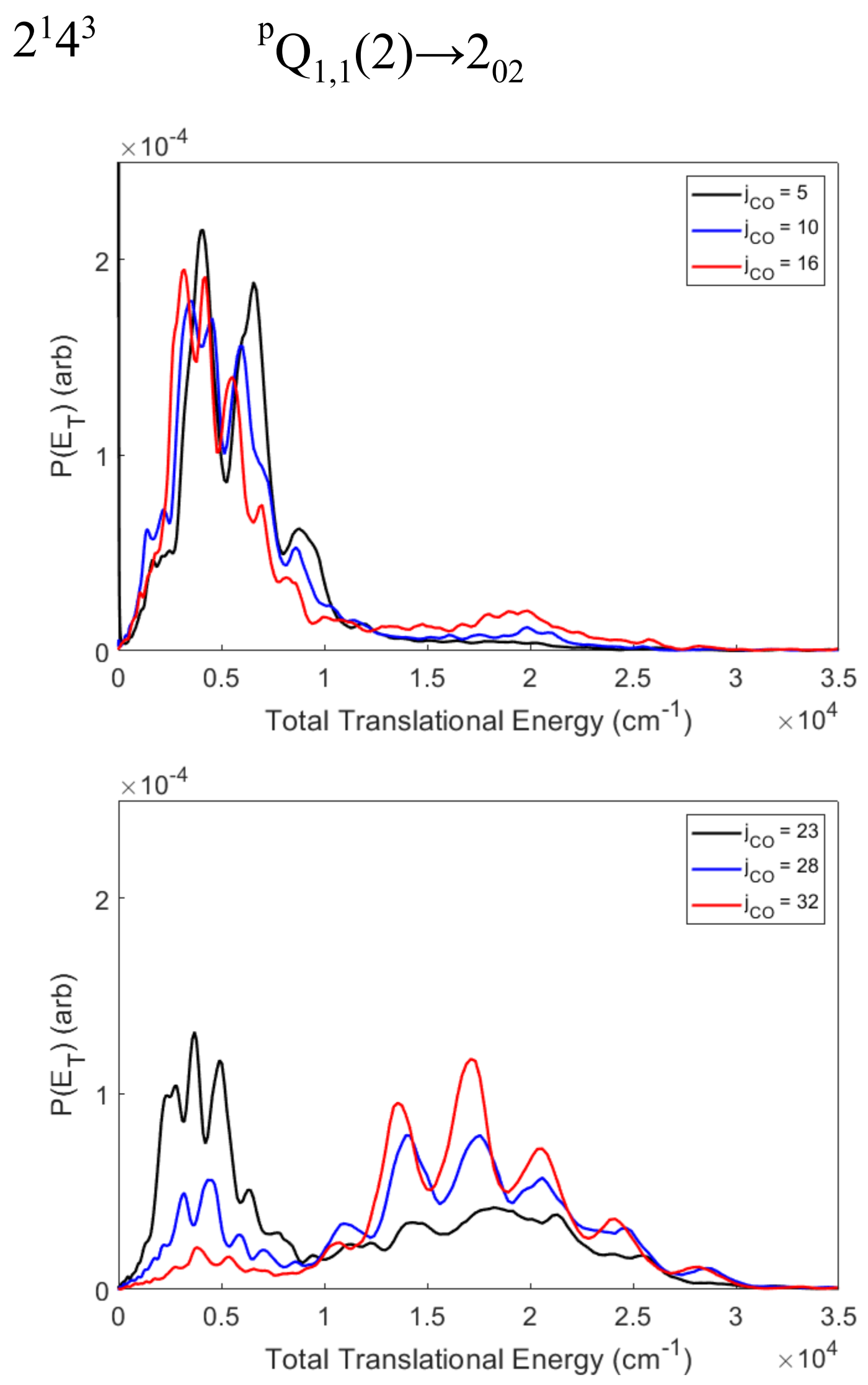
$2^{1} 4^{3}$

${ }^{\mathrm{p}} \mathrm{Q}_{1,1}(2) \rightarrow 2_{02}$

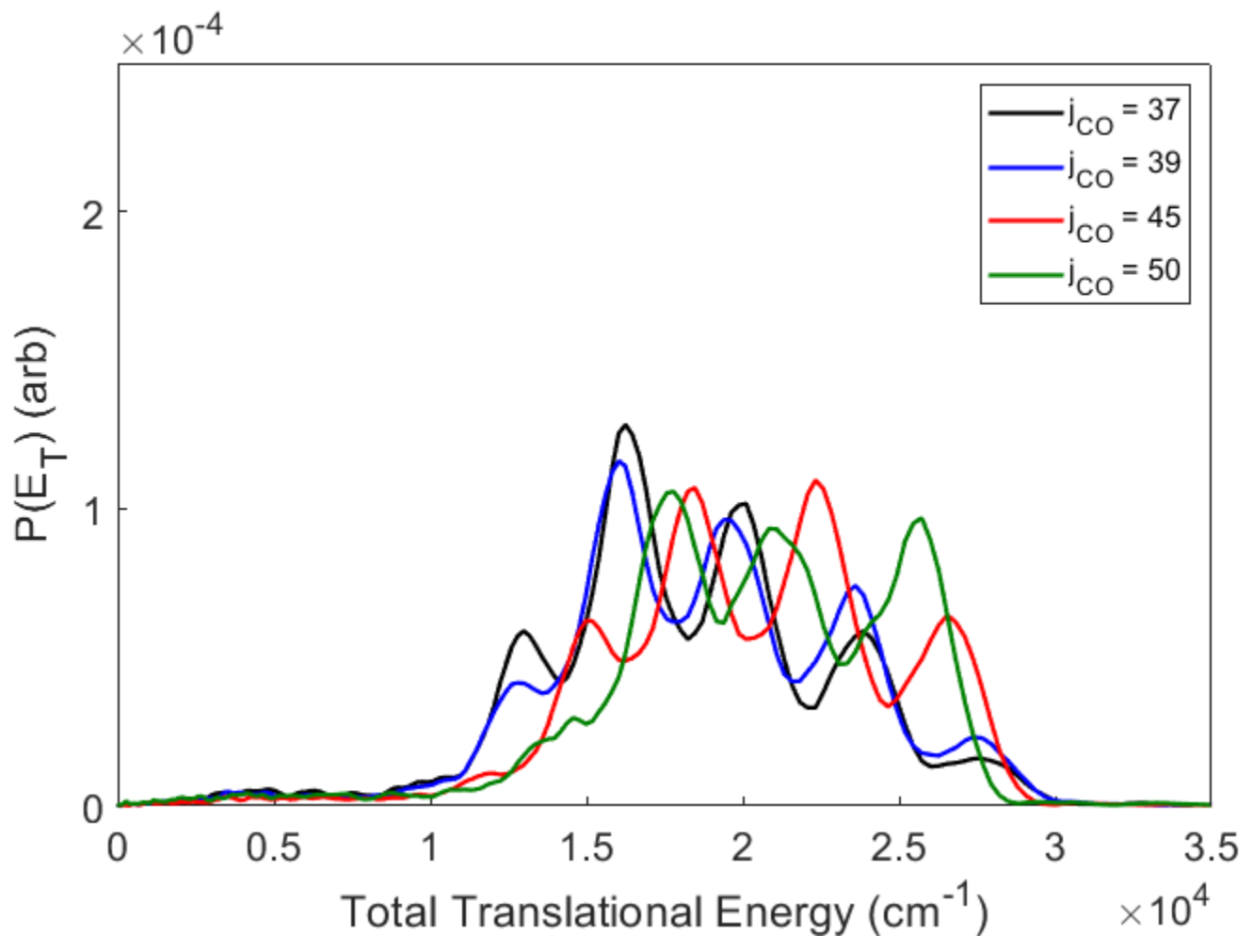



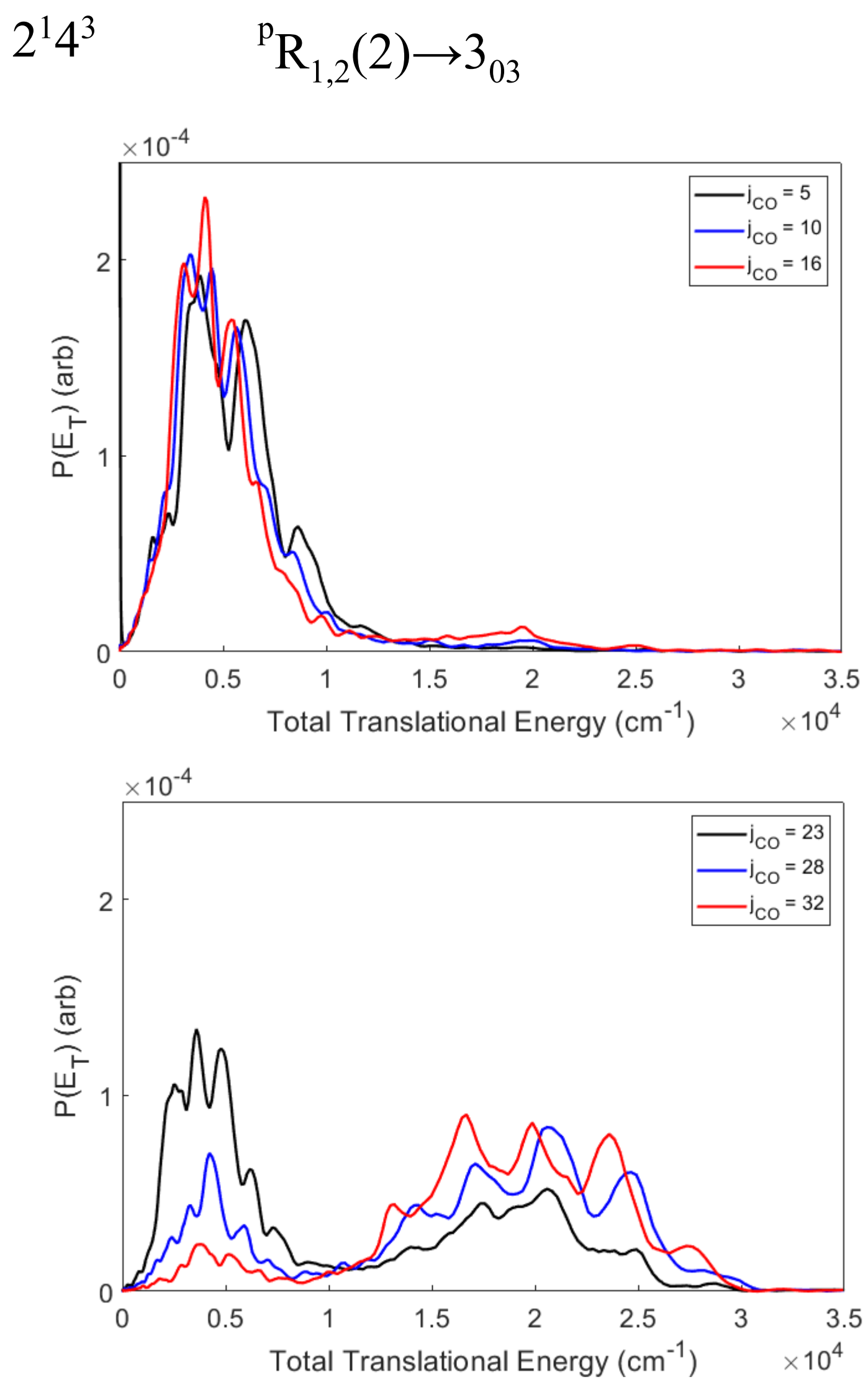
$2^{1} 4^{3}$

${ }^{\mathrm{p}} \mathrm{R}_{1,2}(2) \rightarrow 3_{03}$

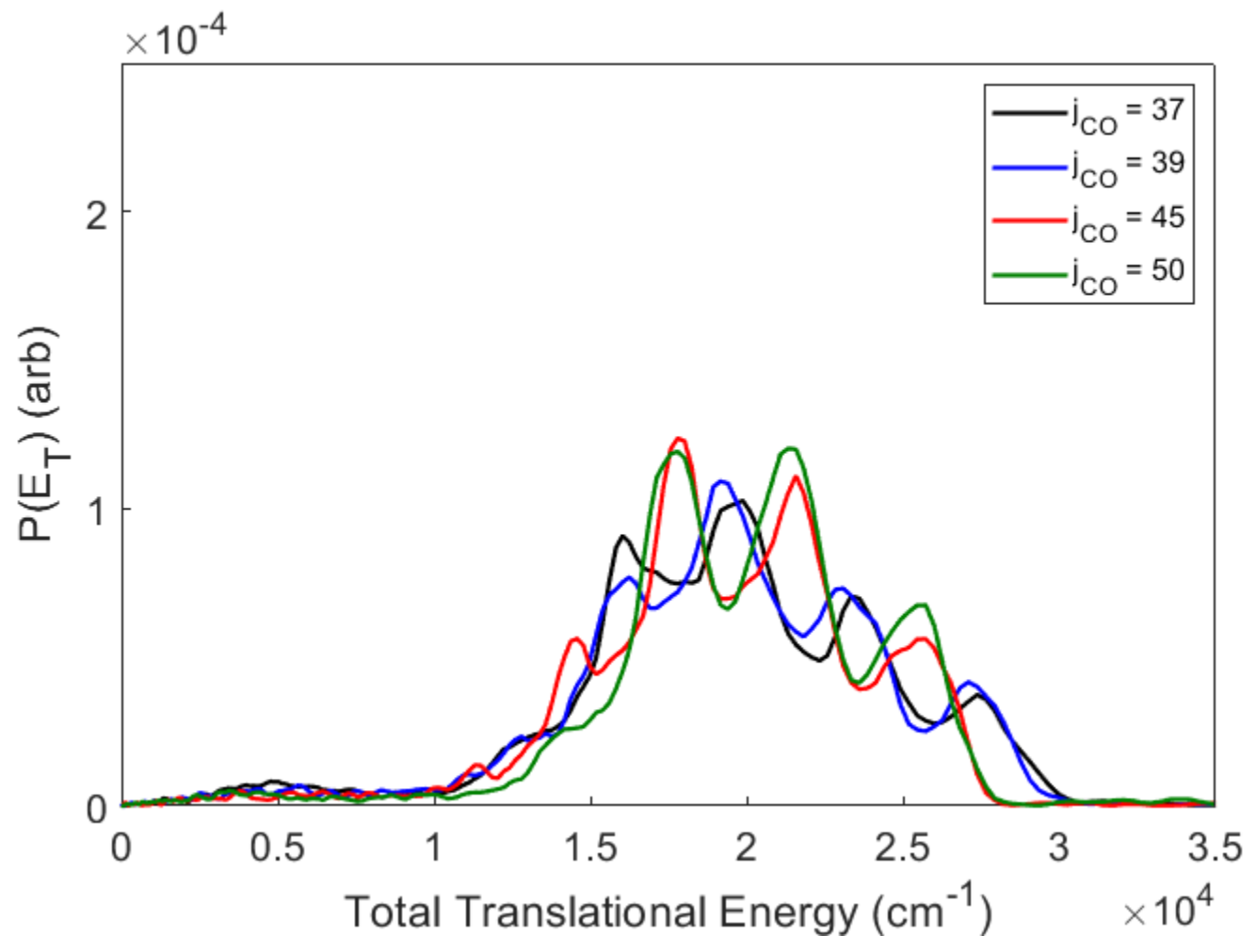


$2^{1} 4^{3} \quad{ }^{\mathrm{p}} \mathrm{P}_{1,5}(5) \rightarrow 4_{04}$

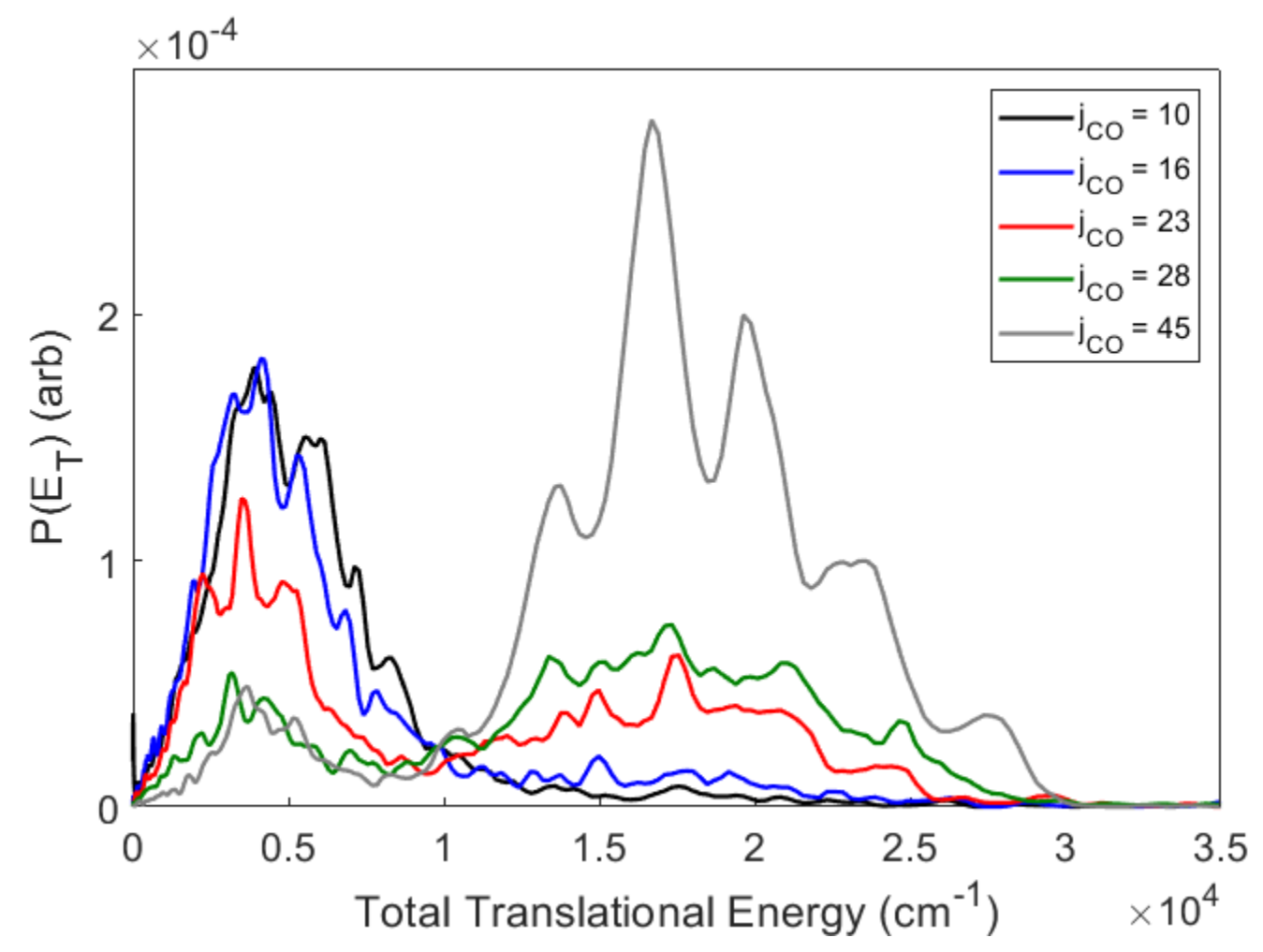



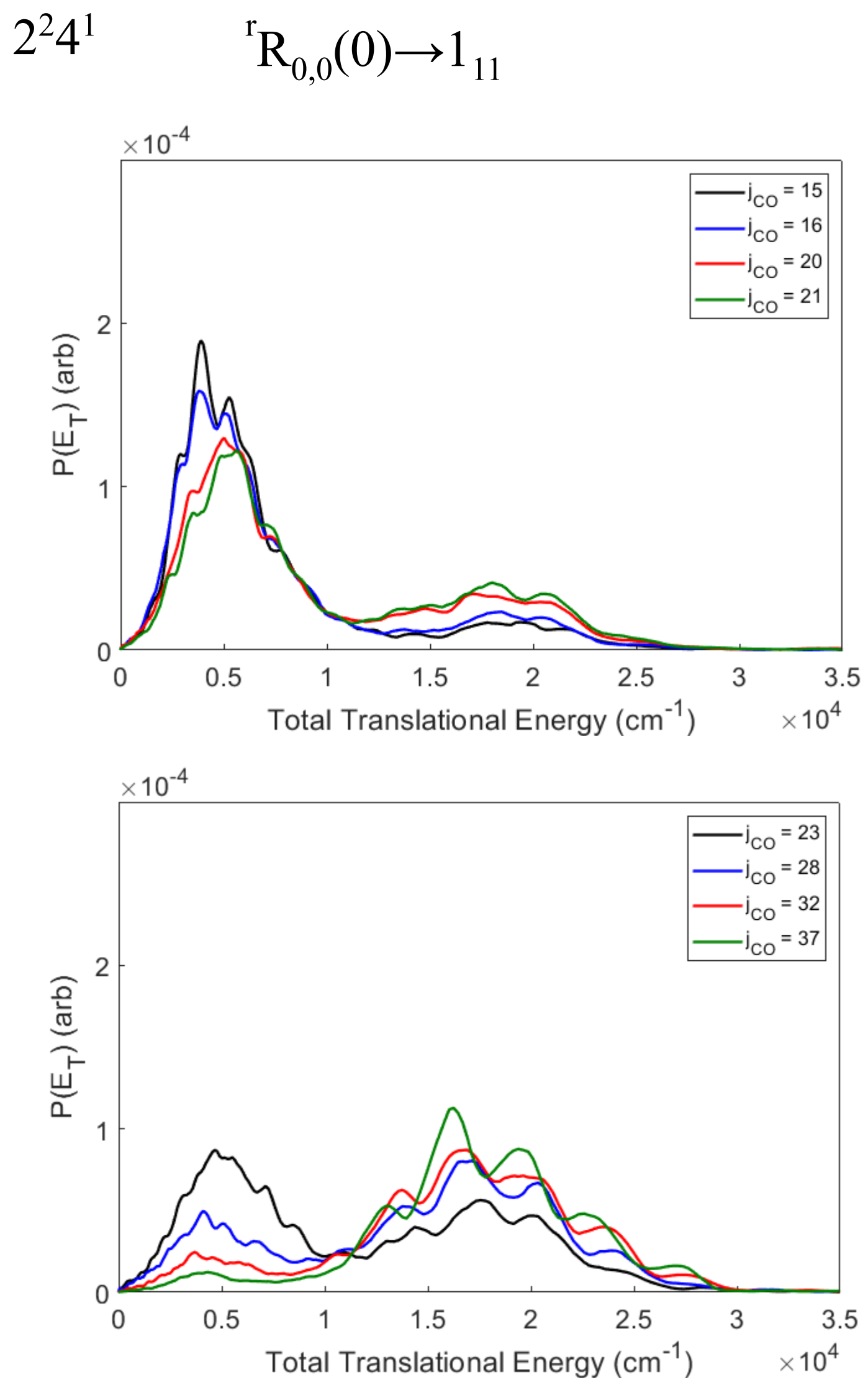

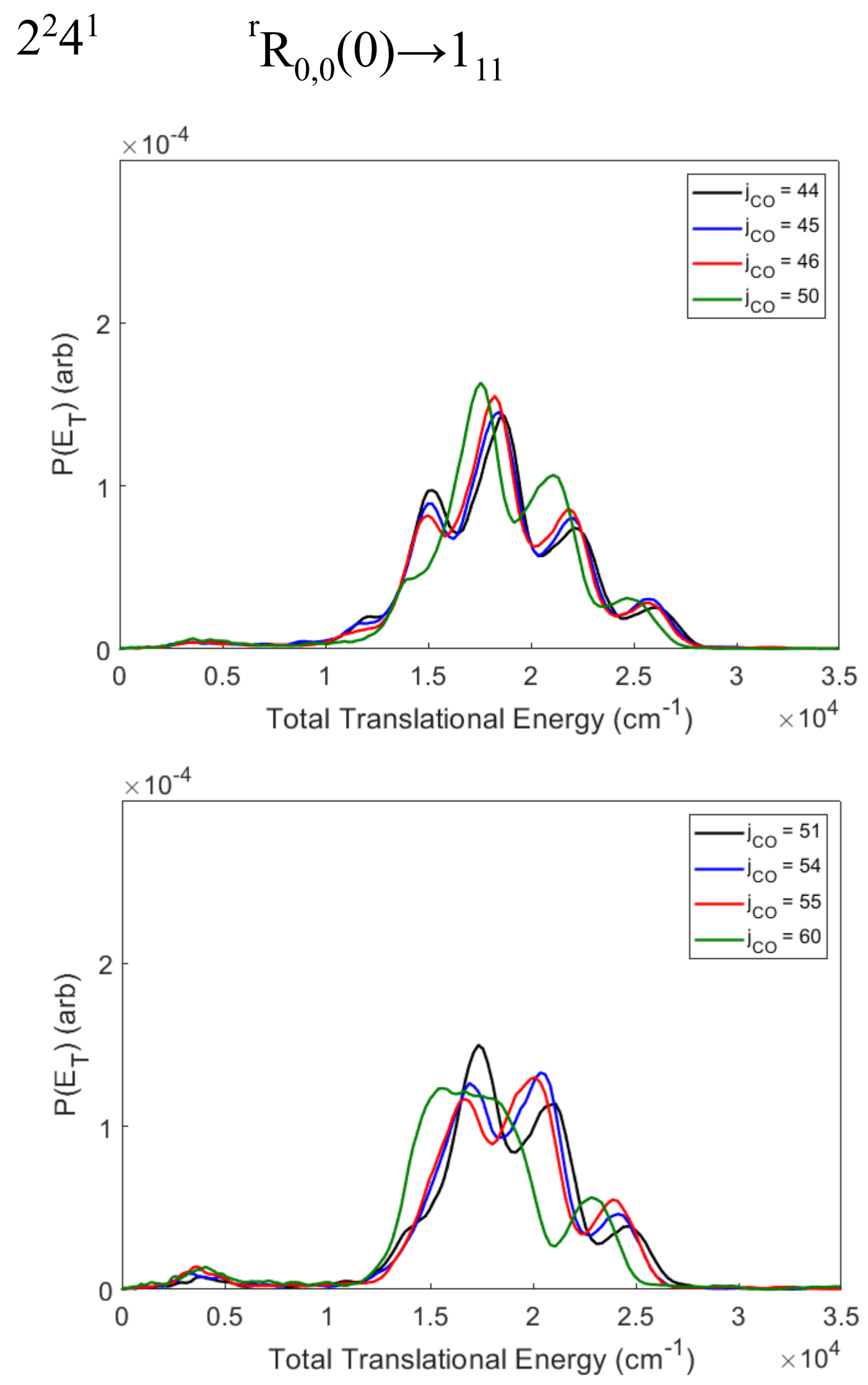

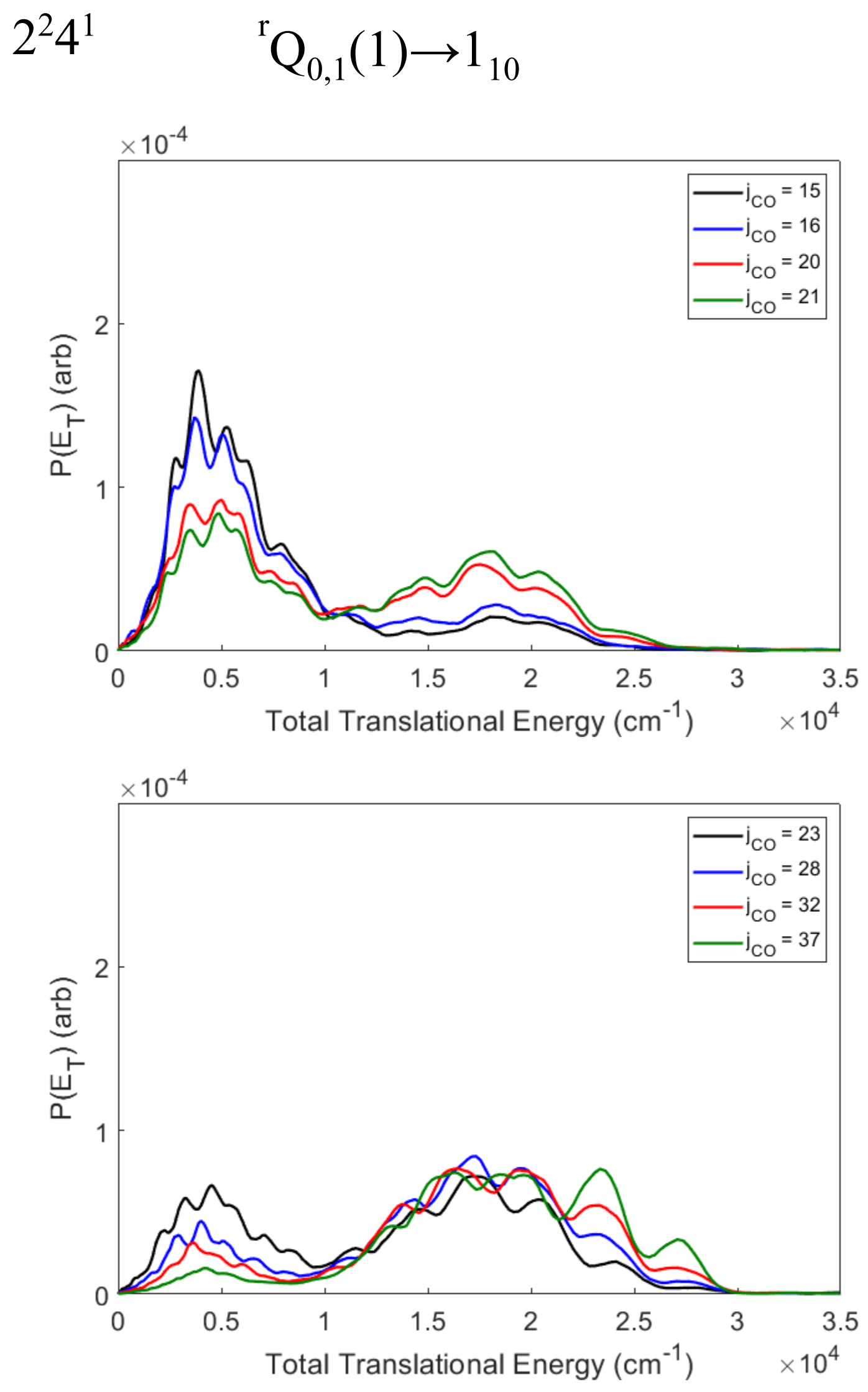

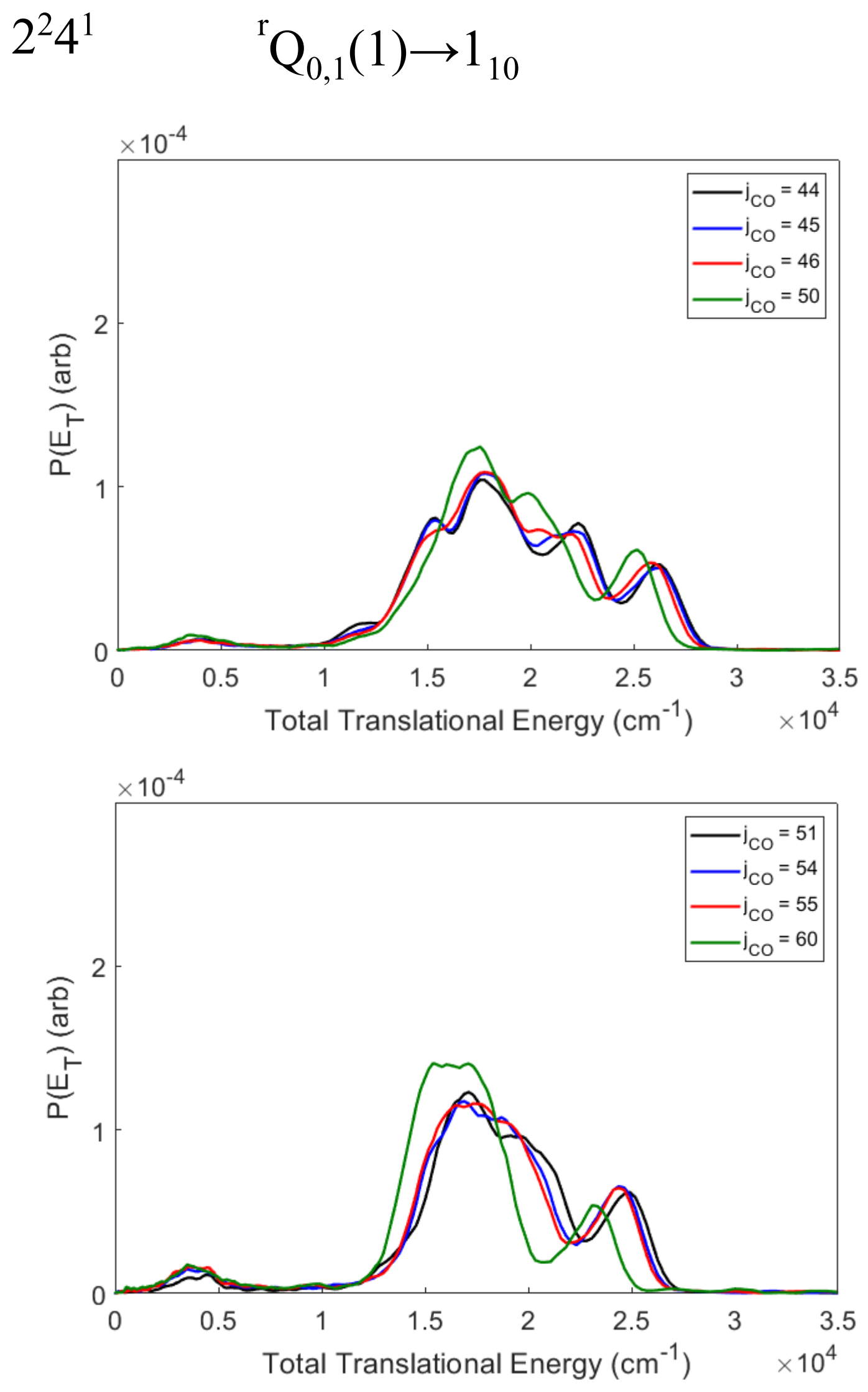

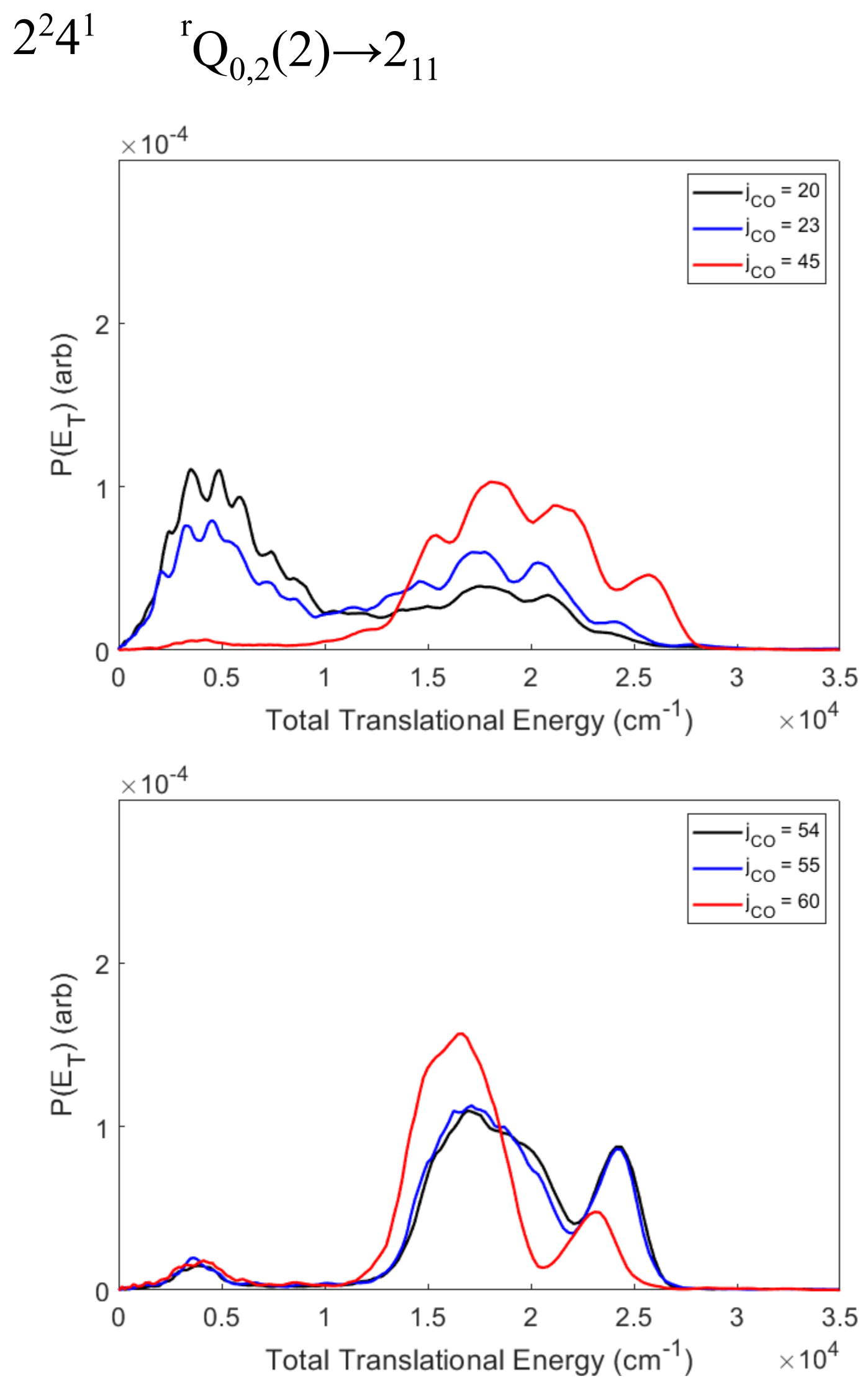

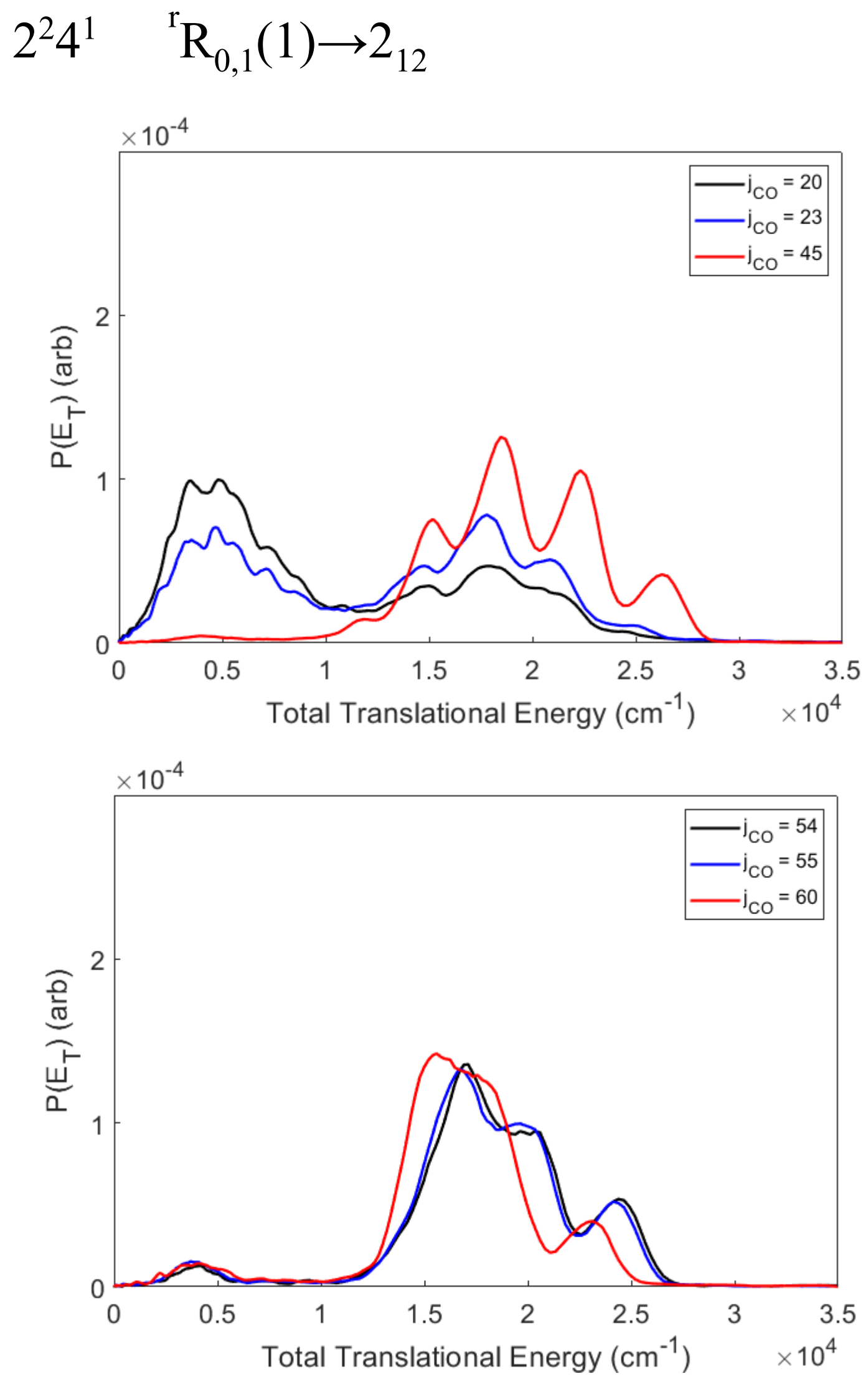

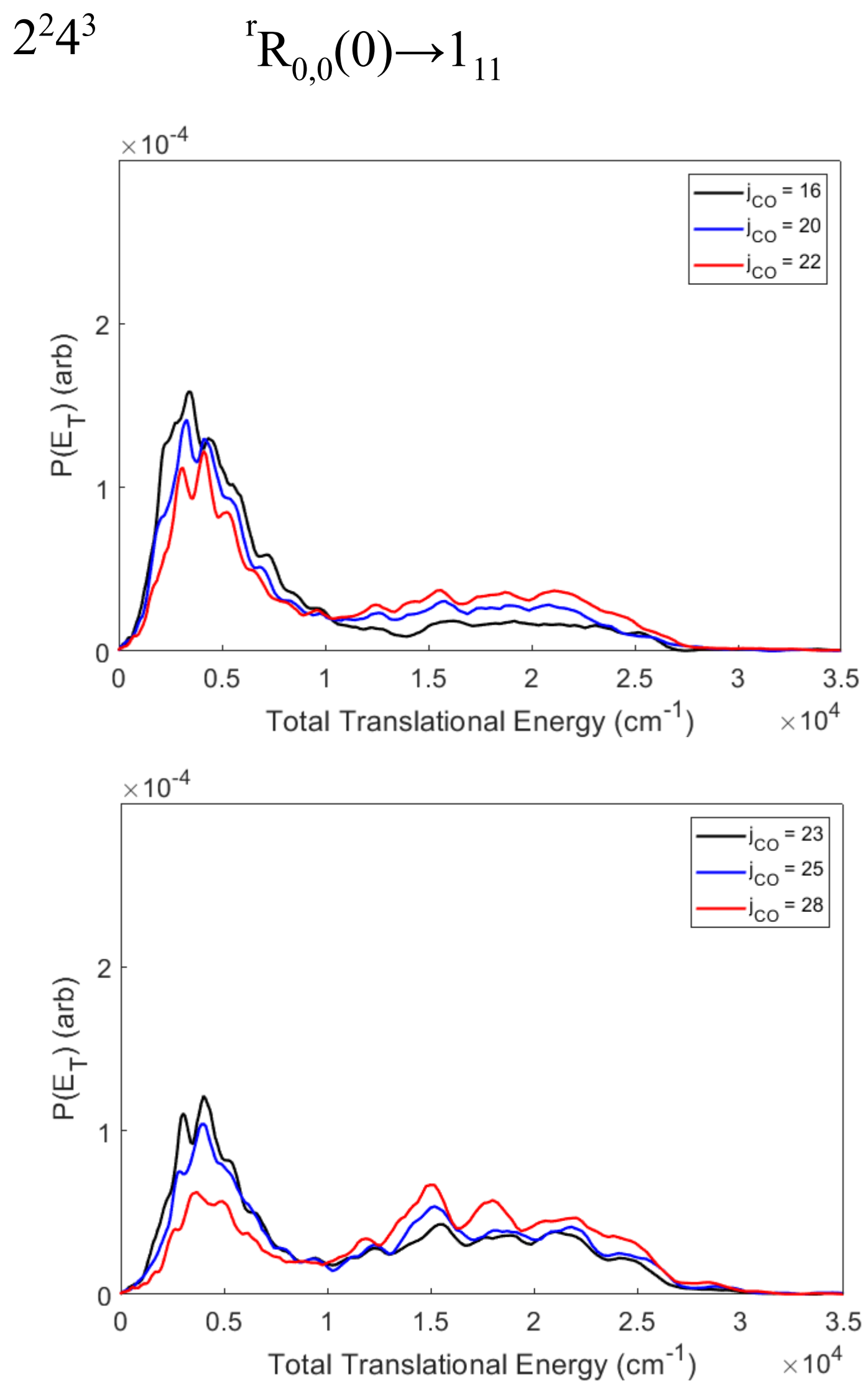

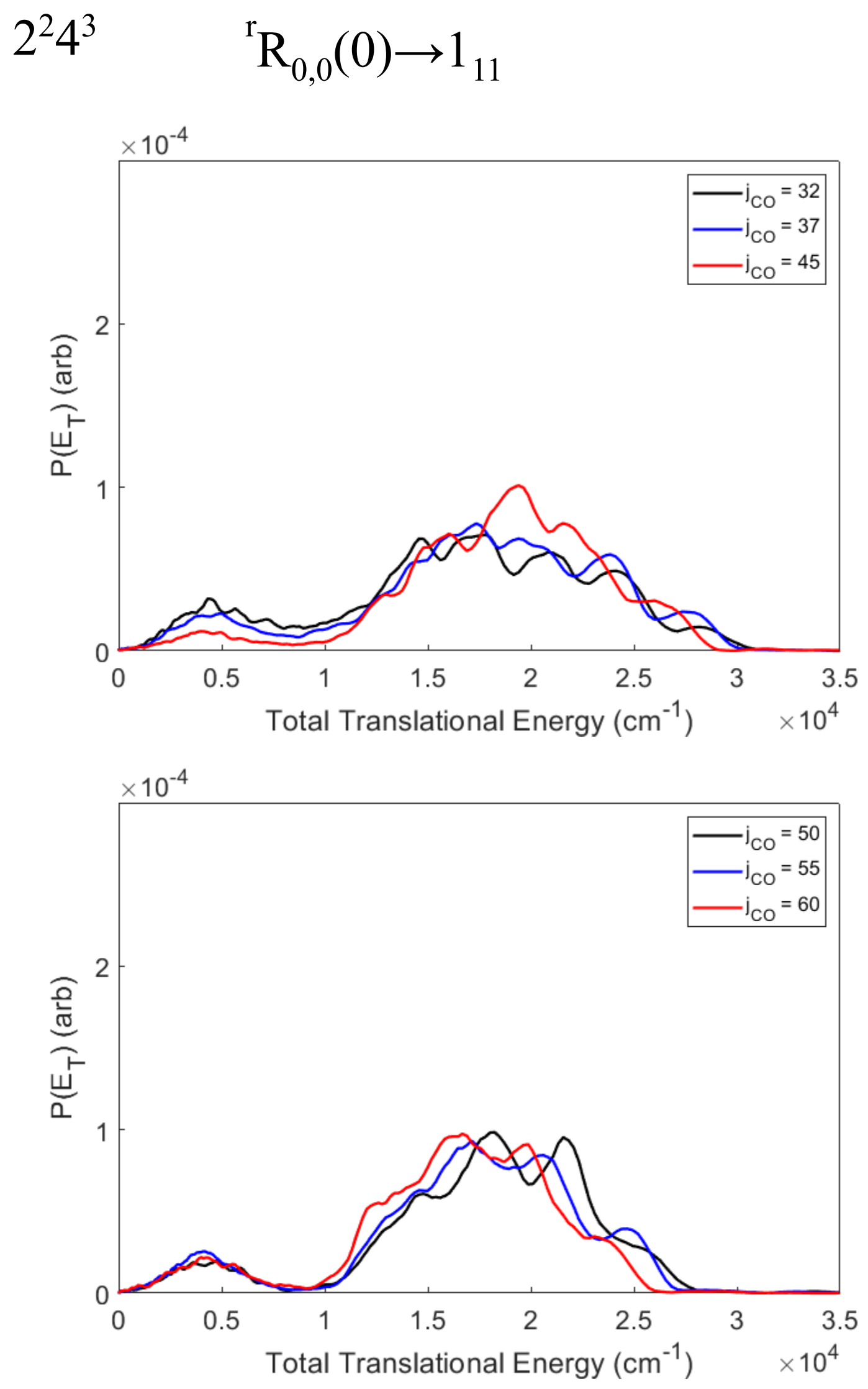

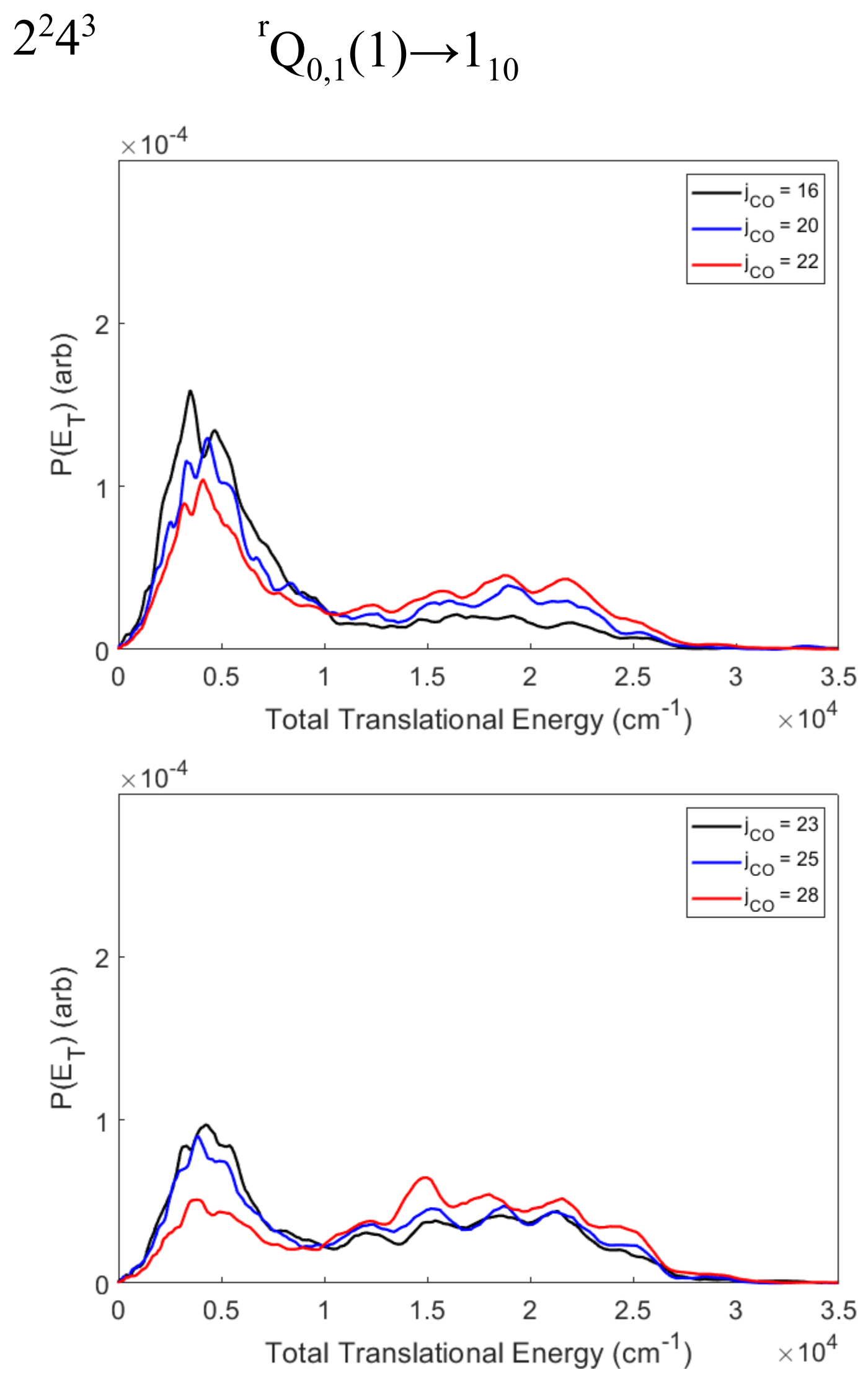

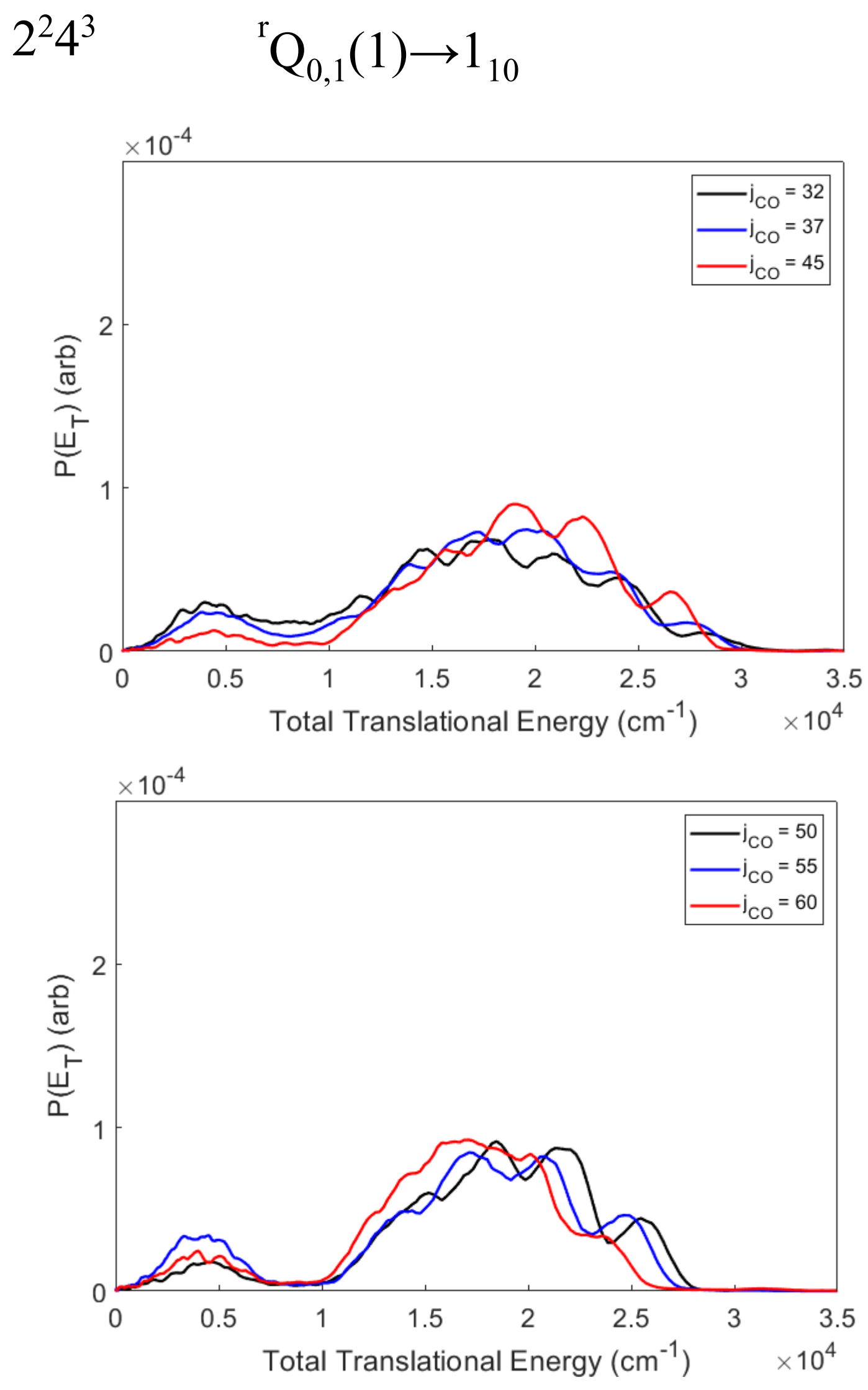

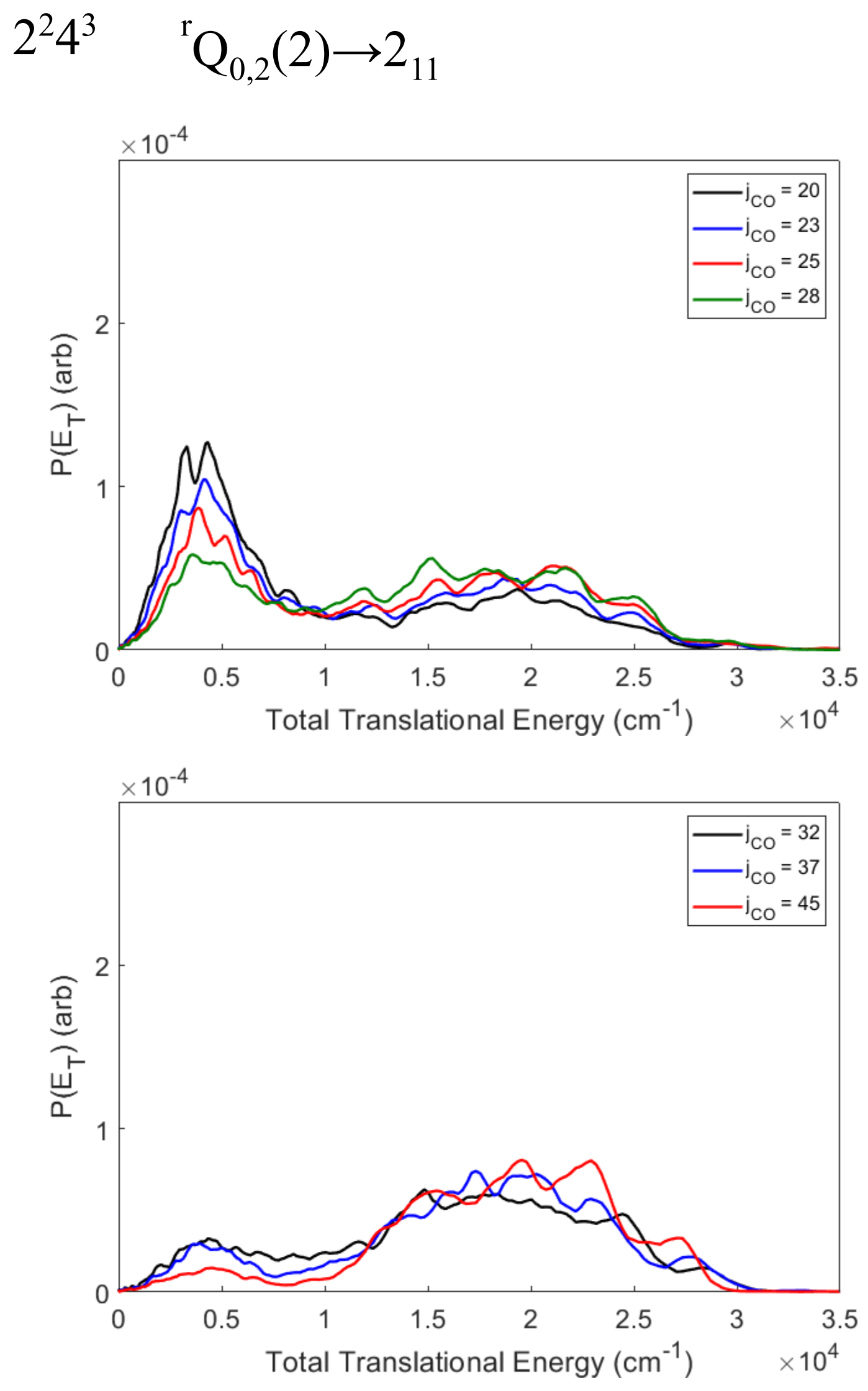

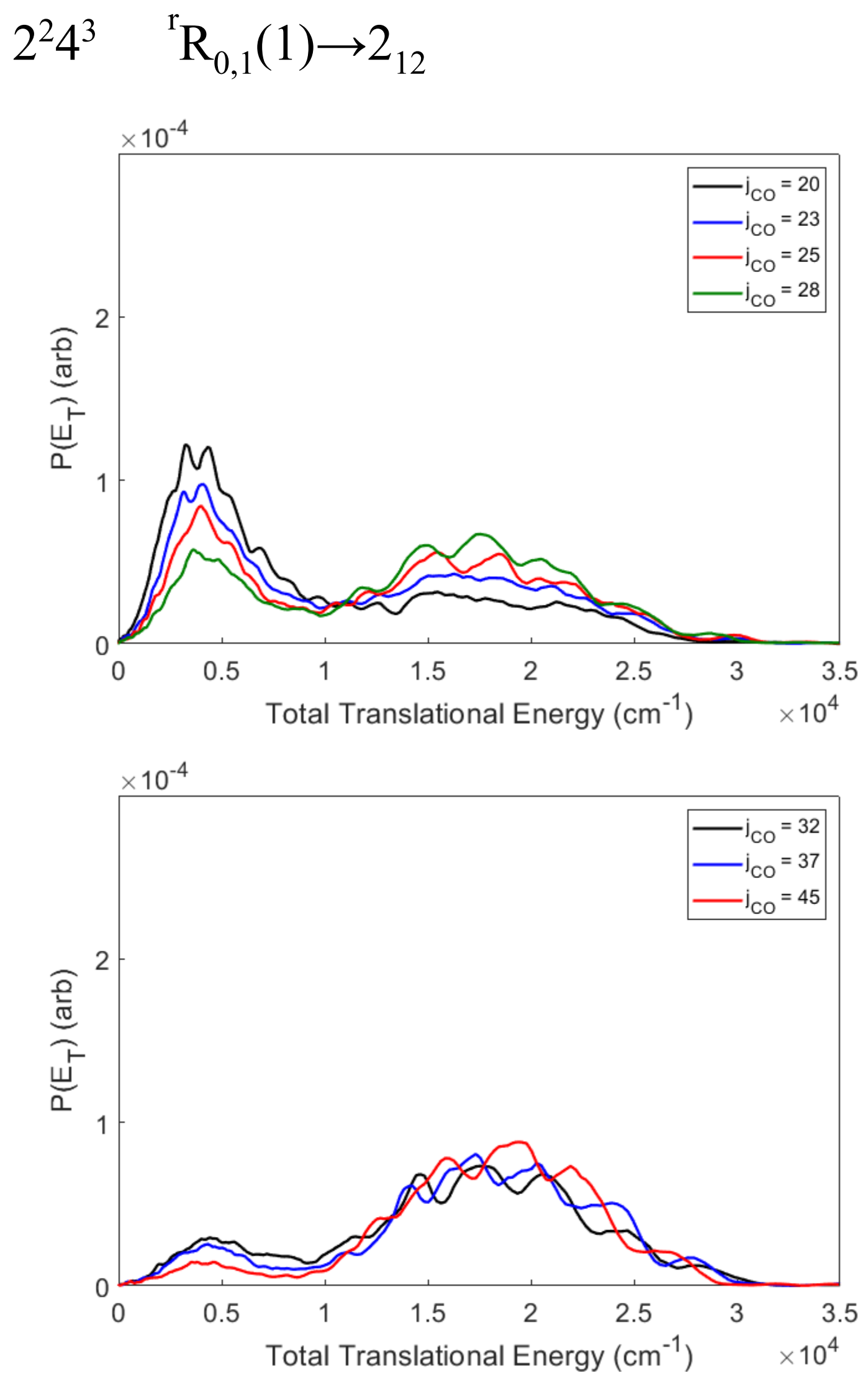


\section{APPENDIX E}

\section{$\mathrm{H}_{2}$ CO PHOFEX spectrum, $\mathrm{J}=44 \mathrm{CO}$}

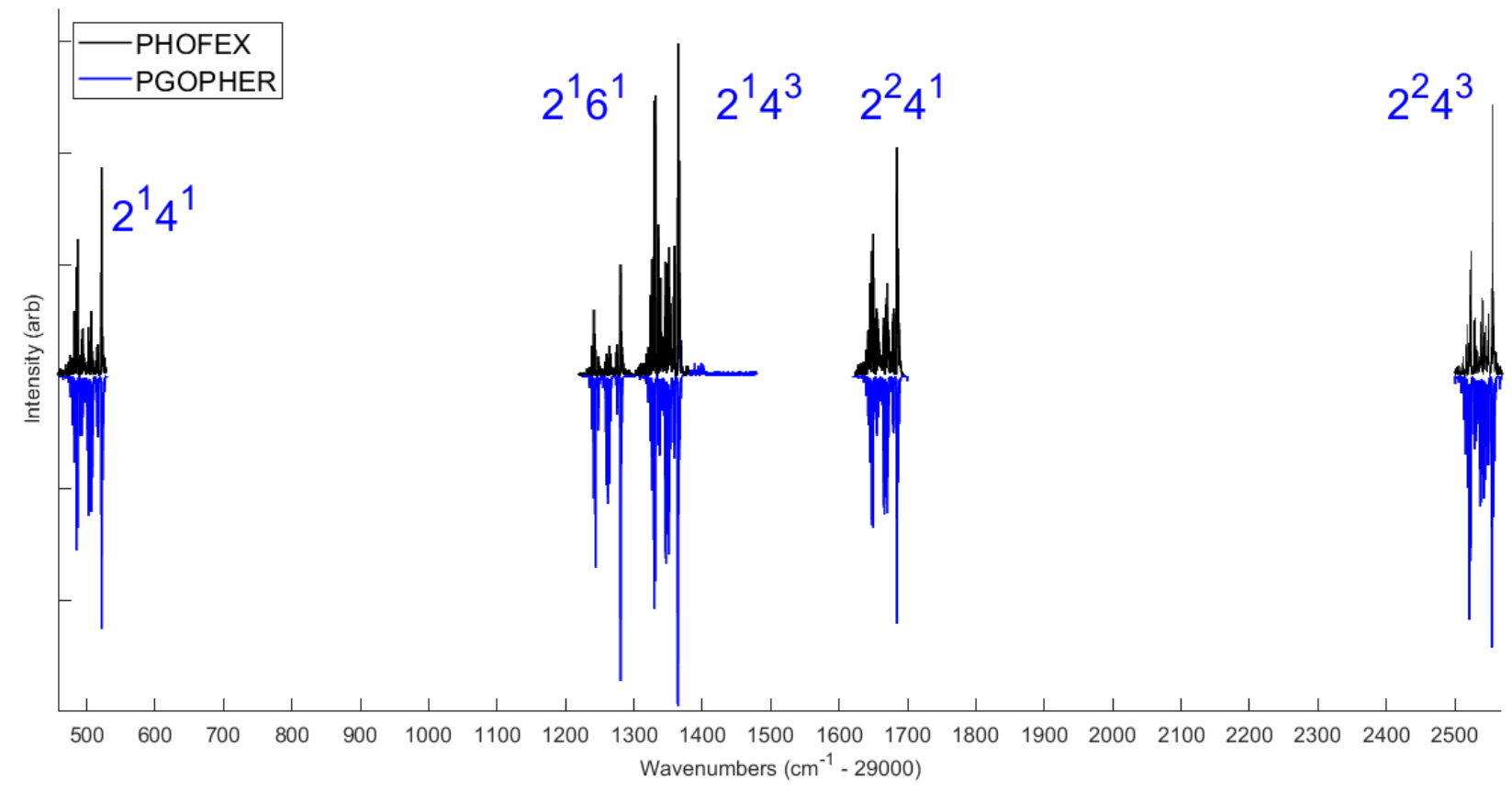

PHOFEX spectrum showing range of energy investigated.

$\mathrm{H}_{2}$ CO PHOFEX spectrum, $\mathrm{J}=44 \mathrm{CO}$

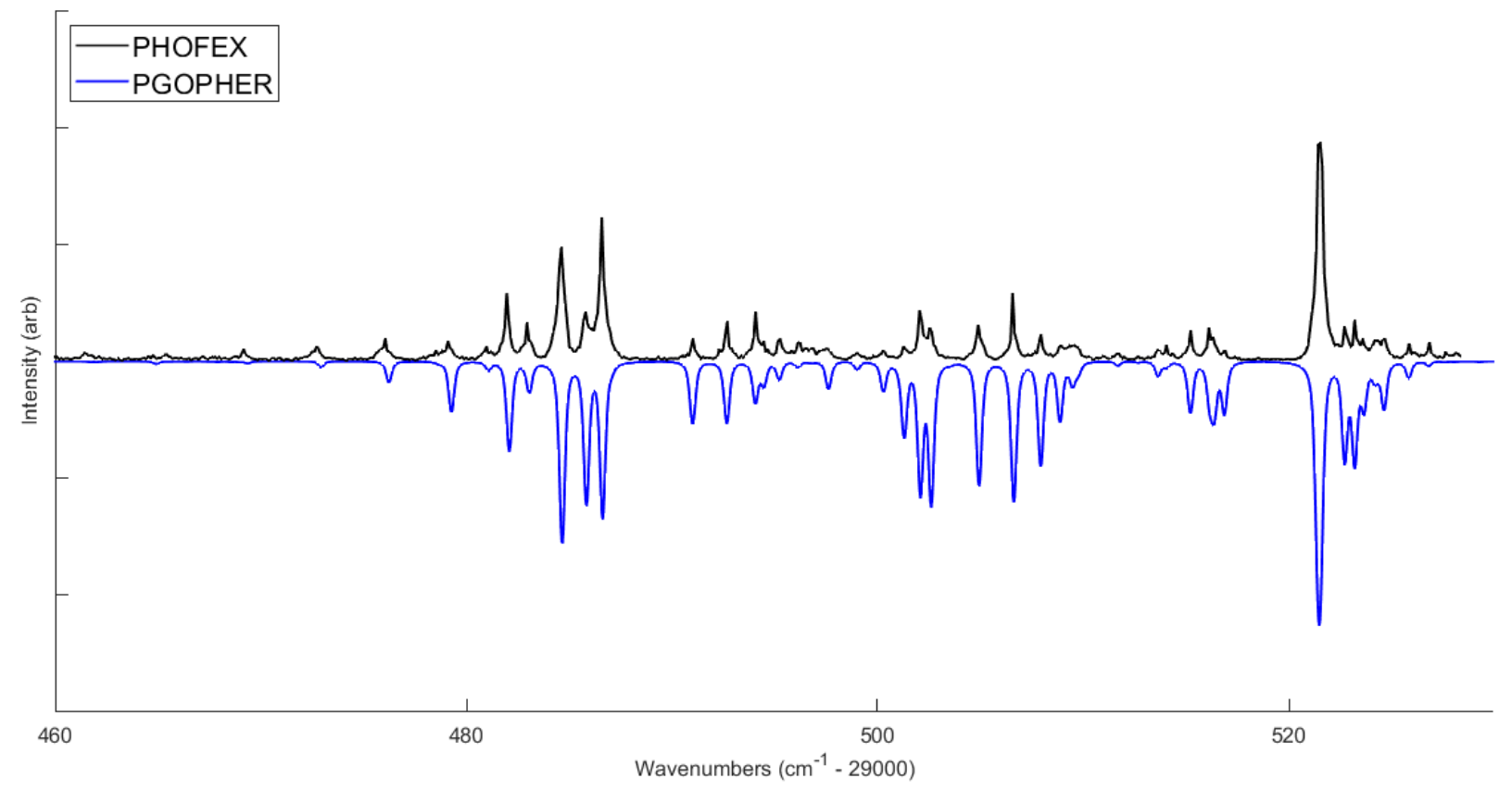

$2^{1} 4^{1}$ PHOFEX spectrum. 
$\mathrm{H}_{2} \mathrm{CO}$ PHOFEX spectrum, $\mathrm{J}=44 \mathrm{CO}$

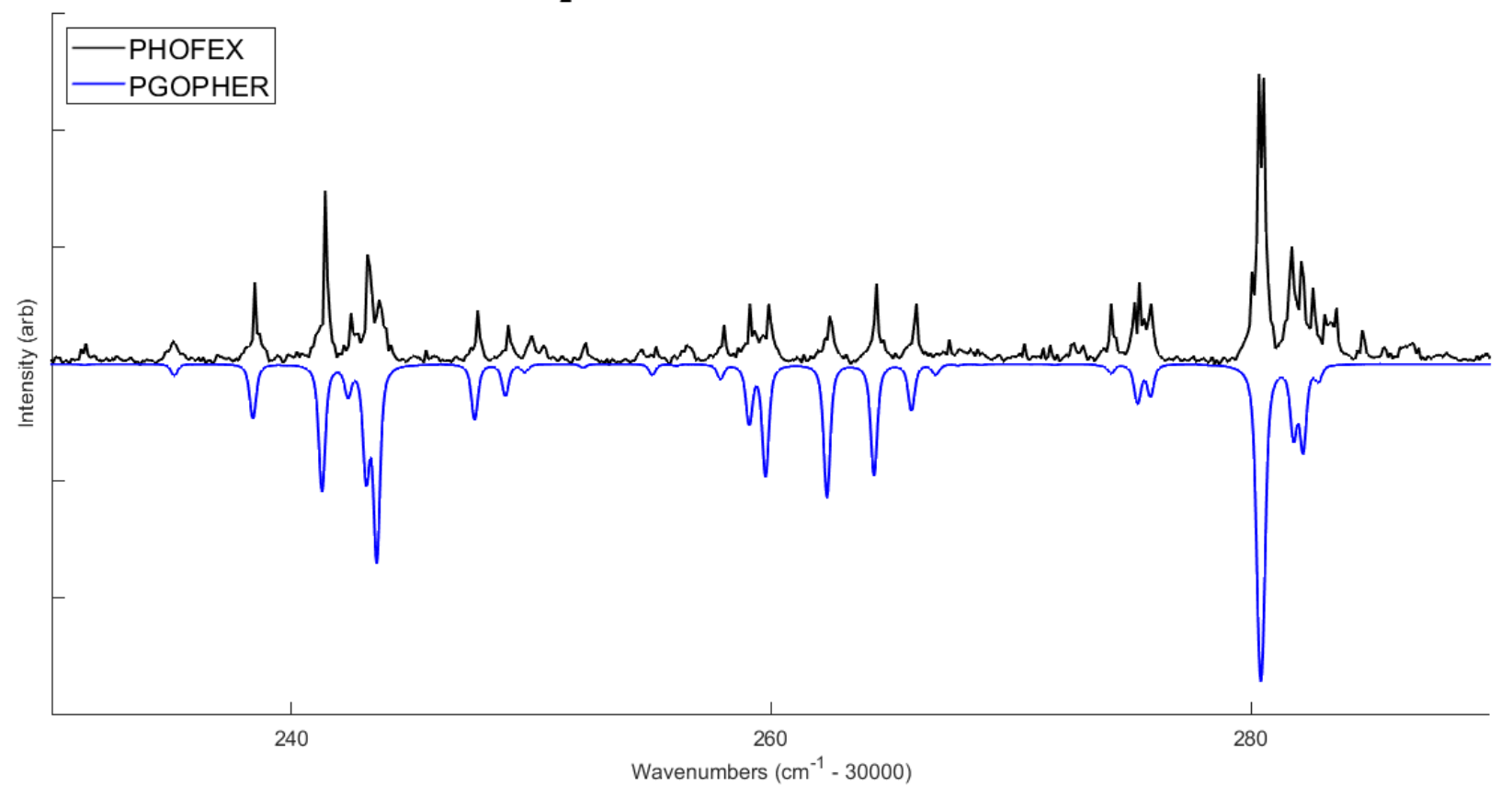

$2^{1} 6^{1}$ PHOFEX spectrum.

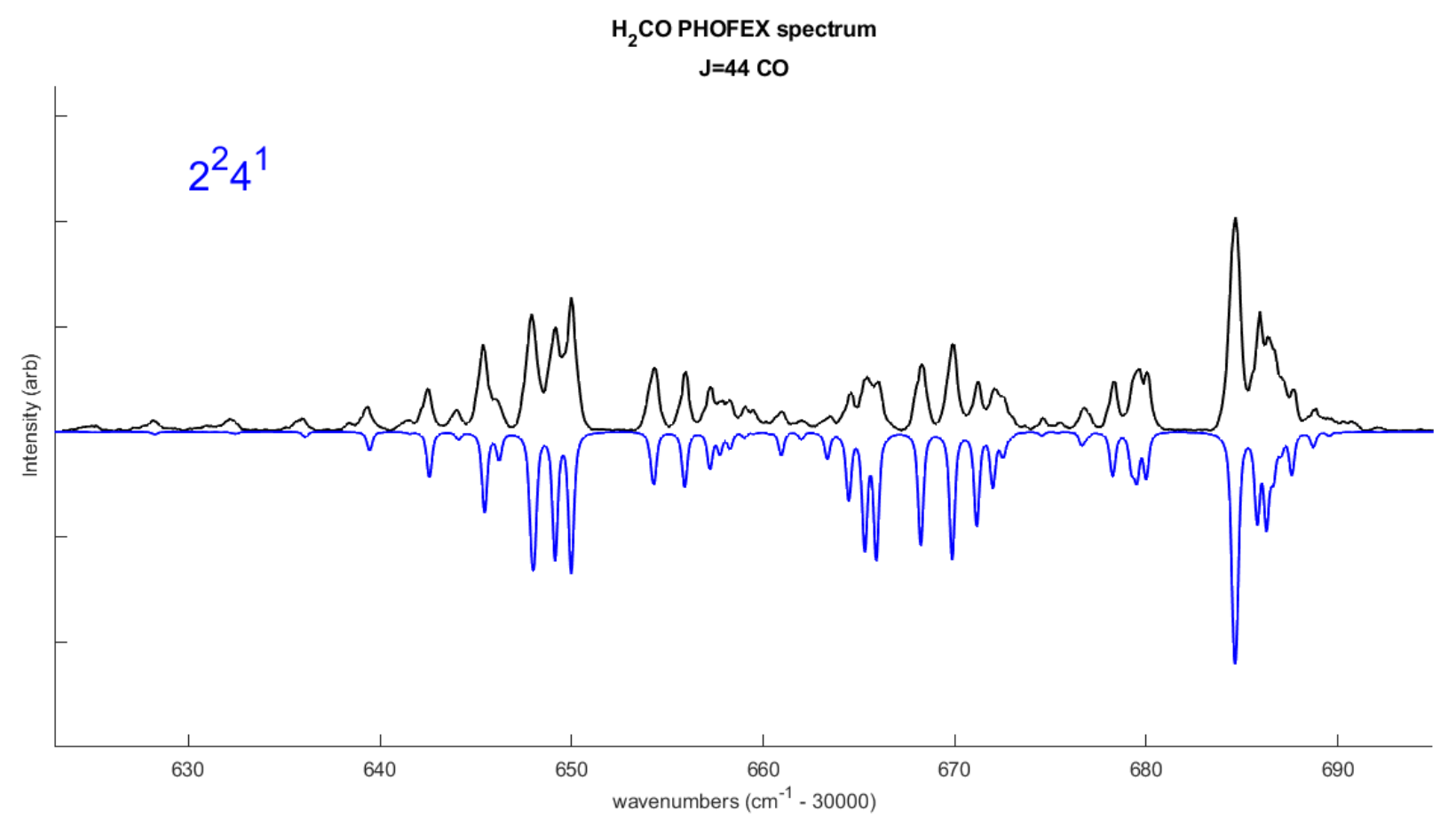

$2^{2} 4^{1}$ PHOFEX spectrum. 


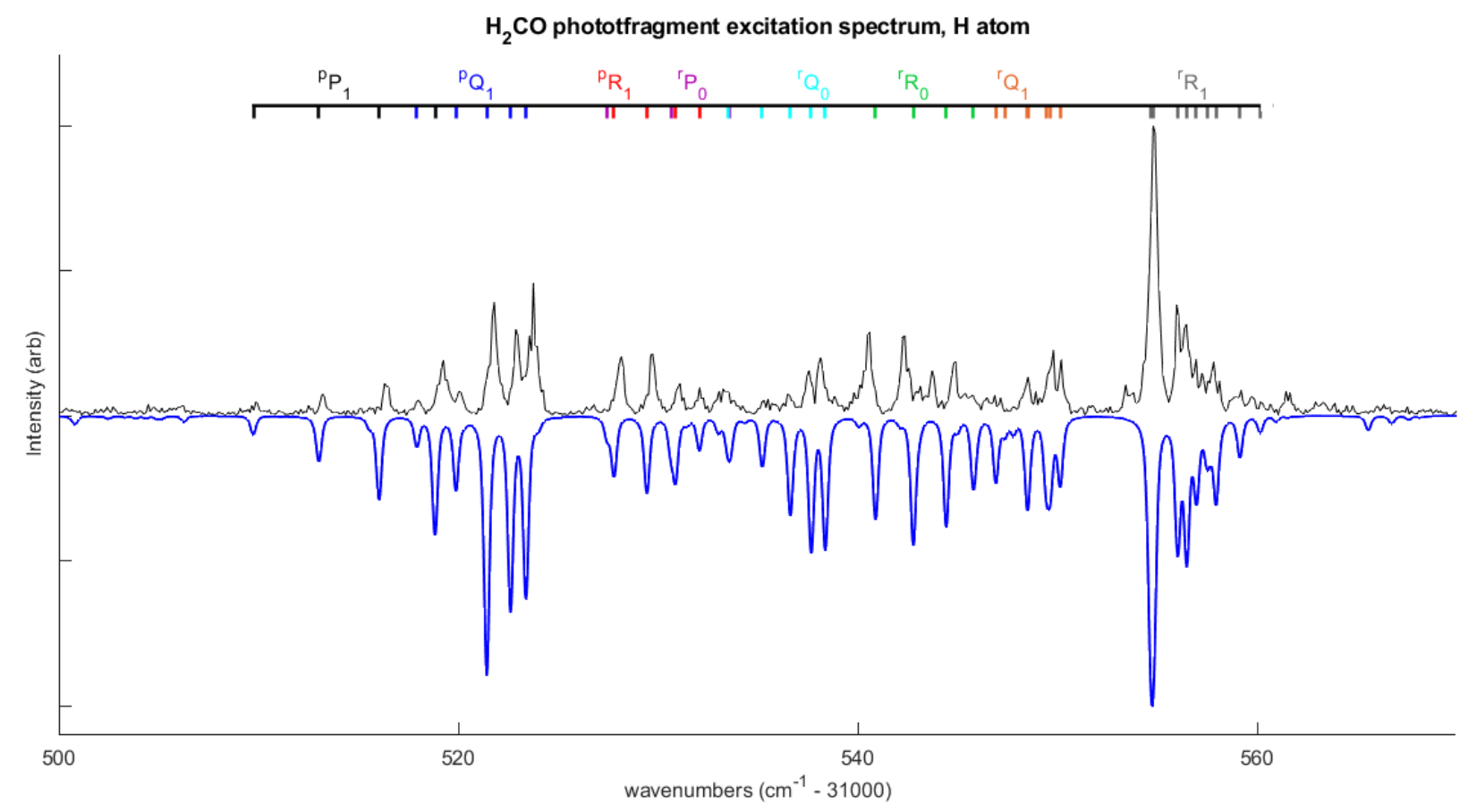

$2^{2} 4^{3}$ PHOFEX spectrum. 


\section{BIBLIOGRAPHY}

[1] B. Ruscic and D. Bross, Active Thermochemical Tables (ATcT) based on ver. 1.122p of the Thermochemical Network (2020). 〈ATcT.anl.gov>

[2] B. Ruscic, R. E. Pinzon, M. L. Morton, G. von Laszevski, S. J. Bittner, S. G. Nijsure, K. A. Amin, M. Minkoff and A. F. Wagner, J. Phys. Chem. A 108, 9979 (2004).

[3] A. Kramida, Yu. Ralchenko, J. Reader and N. A. Team, (\{NIST Atomic Spectra Database (ver. 5.8), [Online]. Available: $\{\backslash \operatorname{tt}\{$ https://physics.nist.gov/asd $\}\}$ [2020, Nov 9]. National Institute of Standards and Technology, Gaithersburg, MD.\}, 2020).

[4] H. Pickett, R. Poynter, E. Cohen, M. Delitsky, J. Pearson and H. Müller, Journal of Quantitative Spectroscopy and Radiative Transfer 60, 883 (1998).

[5] I. E. Gordon, L. S. Rothman, C. Hill, R. V. Kochanov, Y. Tan, P. F. Bernath, M. Birk, V. Boudon, A. Campargue and K. Chance, Journal of Quantitative Spectroscopy and Radiative Transfer 203, 3 (2017).

[6] H. Keller-Rudek, G. Moortgat, R. Sander and R. Sörensen, Earth System Science Data Discussions 6 (2013).

[7] P. Linstrom and W. Mallard (National Institute of Standards and Technology, Gaithersburg MD, 20899, 2020).

[8] C. M. Western, J. Quant. Spectrosc. Ra. 186, 221 (2017).

[9] D. R. Herschbach, Angewandte Chemie International Edition in English 26, 1221 (1987).

[10] Y. T. Lee, Angewandte Chemie International Edition in English 26, 939 (1987).

[11] J. C. Polanyi, Angewandte Chemie International Edition in English 26, 952 (1987).

[12] O. K. Rice and H. C. Ramsperger, J. Am. Chem. Soc. 49, 1617 (1927).

[13] O. K. Rice and H. C. Ramsperger, J. Am. Chem. Soc. 50, 617 (1928).

[14] L. Kassel, J. Phys. Chem. 32, 1065 (2002).

[15] L. S. Kassel, J. Phys. Chem. 32, 225 (2002).

[16] G. M. Wieder and R. Marcus, J. Chem. Phys. 37, 1835 (1962).

[17] D. G. Truhlar, B. C. Garrett and S. J. Klippenstein, J. Phys. Chem. 100, 12771 (1996).

[18] S. R. Vande Linde and W. L. Hase, Journal of Physical Chemistry 94, 6148 (1990).

[19] D. L. Bunker and W.-L. Hase, J. Chem. Phys. 59, 4621 (1973). 
[20] D. H. Semmes, J. S. Baskin and A. H. Zewail, J. Am. Chem. Soc. 109, 4104 (1987).

[21] A. Li, J. Li and H. Guo, J. Phys. Chem. A 117, 5052 (2013).

[22] F. d. r. A. Mauguière, P. Collins, S. Stamatiadis, A. Li, G. S. Ezra, S. C. Farantos, Z. C. Kramer, B. K. Carpenter, S. Wiggins and H. Guo, J. Phys. Chem. A 120, 5145 (2016).

[23] J. M. Bowman and P. L. Houston, Chemical Society Reviews 46, 7615 (2017).

[24] D. Townsend, S. Lahankar, S. Lee, S. Chambreau, A. Suits, X. Zhang, J. Rheinecker, L. Harding and J. Bowman, Science 306, 1158 (2004).

[25] S. A. Lahankar, S. D. Chambreau, X. Zhang, J. M. Bowman and A. G. Suits, J. Chem. Phys. 126, 044314 (2007).

[26] Z. Homayoon and J. M. Bowman, J. Phys. Chem. A 117, 11665 (2013).

[27] X. Zhang, J. L. Rheinecker and J. M. Bowman, J. Chem. Phys. 122, 114313 (2005).

[28] L. B. Harding and S. J. Klippenstein, J. Phys. Chem. Lett. 1, 3016 (2010).

[29] L. B. Harding, S. J. Klippenstein and A. W. Jasper, J. Phys. Chem. A 116, 6967 (2012).

[30] L. B. Harding, S. J. Klippenstein and A. W. Jasper, Phys. Chem. Chem. Phys. 9, 4055 (2007).

[31] S. J. Klippenstein, Y. Georgievskii and L. B. Harding, J. Phys. Chem. A 115, 14370 (2011).

[32] F. A. Mauguière, P. Collins, G. S. Ezra, S. C. Farantos and S. Wiggins, J. Chem. Phys. 140, 134112 (2014).

[33] F. A. Mauguière, P. Collins, Z. C. Kramer, B. K. Carpenter, G. S. Ezra, S. C. Farantos and S. Wiggins, J. Chem. Phys. 144, 054107 (2016).

[34] F. A. Mauguière, P. Collins, Z. C. Kramer, B. K. Carpenter, G. S. Ezra, S. C. Farantos and S. Wiggins, Ann. Rev. Phys. Chem. 68, 499 (2017).

[35] D. U. Andrews, S. H. Kable and M. J. Jordan, J. Phys. Chem. A 117, 7631 (2013).

[36] T. Takayanagi and T. Tanaka, Chem. Phys. Lett. 504, 130 (2011).

[37] R. Fernando, A. Dey, B. M. Broderick, B. Fu, Z. Homayoon, J. M. Bowman and A. G. Suits, J. Phys. Chem. A 119, 7163 (2014).

[38] M. P. Grubb, M. L. Warter, A. G. Suits and S. W. North, J. Phys. Chem. Lett. 1, 2455 (2010).

[39] M. P. Grubb, M. L. Warter, H. Xiao, S. Maeda, K. Morokuma and S. W. North, Science 335, 1075 (2012). 
[40] C. D. Foley, B. Joalland, S. T. Alavi and A. G. Suits, Phys. Chem. Chem. Phys. 20, 27474 (2018).

[41] C. D. Foley, S. T. Alavi, B. Joalland, B. M. Broderick, N. Dias and A. G. Suits, Phys. Chem. Chem. Phys. 21, 1528 (2019).

[42] C. Foley, G. Cooper, J. Tu, M. Harmata and A. Suits, Molecular Physics, 1 (2020).

[43] A. G. Suits, Acc. Chem. Res 41, 873 (2008).

[44] A. G. Suits, Ann. Rev. Phys. Chem. 71, 77 (2020).

[45] R. G. Norrish, Transactions of the Faraday Society 30, 103 (1934).

[46] F. Mohammad, V. R. Morris, W. H. Fink and W. M. Jackson, J. Phys. Chem. 97, 11590 (1993).

[47] T. P. Marcy, R. R. Díaz, D. Heard, S. R. Leone, L. B. Harding and S. J. Klippenstein, J. Phys. Chem. A 105, 8361 (2001).

[48] L. Sun, K. Song and W. L. Hase, Science 296, 875 (2002).

[49] J. G. López, G. Vayner, U. Lourderaj, S. V. Addepalli, S. Kato, W. A. deJong, T. L. Windus and W. L. Hase, J. Am. Chem. Soc. 129, 9976 (2007).

[50] H. E. Audier and T. H. Morton, Organic mass spectrometry 28, 1218 (1993).

[51] R. D. van Zee, M. F. Foltz and C. B. Moore, J. Chem. Phys. 99, 1664 (1993).

[52] S. Lahankar, V. Goncharov, F. Suits, J. Farnum, J. Bowman and A. G. Suits, Chem. Phys. 347, 288 (2008).

[53] M. S. Quinn, D. U. Andrews, K. Nauta, M. J. Jordan and S. H. Kable, J. Chem. Phys. 147, 013935 (2017).

[54] P. L. Houston, R. Conte and J. M. Bowman, J. Phys. Chem. A 120, 5103 (2016).

[55] P. L. Houston and S. H. Kable, P. Natl. Acad. Sci. 103, 16079 (2006).

[56] P.-Y. Tsai, K.-C. Hung, H.-K. Li and K.-C. Lin, J. Phys. Chem. Lett. 5, 190 (2014).

[57] V. Goncharov, N. Herath and A. G. Suits, J. Phys. Chem. A 112, 9423 (2008).

[58] B. Fu, J. M. Bowman, H. Xiao, S. Maeda and K. Morokuma, Journal of chemical theory and computation 9, 893 (2013).

[59] R. Zhu and M. Lin, Chem. Phys. Lett. 478, 11 (2009).

[60] R. Zhu, P. Raghunath and M. Lin, J. Phys. Chem. A 117, 7308 (2013). 
[61] R. Sivaramakrishnan, J. Michael, L. Harding and S. Klippenstein, J. Phys. Chem. A 116, 5981 (2012).

[62] R. Sivaramakrishnan, J. V. Michael, A. F. Wagner, R. Dawes, A. W. Jasper, L. B. Harding, Y. Georgievskii and S. J. Klippenstein, Combustion and Flame 158, 618 (2011).

[63] R. Sivaramakrishnan, M.-C. Su, J. Michael, S. Klippenstein, L. Harding and B. Ruscic, J. Phys. Chem. A 115, 3366 (2011).

[64] A. Vandeputte, L. Harding, Y. Georgievskii and S. Klippenstein, (2013).

[65] R. Sivaramakrishnan, J. Michael and S. Klippenstein, J. Phys. Chem. A 114, 755 (2010).

[66] E. J. K. Nilsson, V. F. Andersen, H. Skov and M. S. Johnson, Atmospheric Chemistry \& Physics 10 (2010).

[67] R. Asatryan, Y. Pal, J. Hachmann and E. Ruckenstein, J. Phys. Chem. A 122, 9738 (2018).

[68] M. L. Hause, N. Herath, R. Zhu, M. Lin and A. G. Suits, Nat. Chem. 3, 932 (2011).

[69] M. Isegawa, F. Liu, S. Maeda and K. Morokuma, J. Chem. Phys. 140, 244310 (2014).

[70] A. Kantrowitz and J. Grey, Rev. Sci. Instrum. 22, 328 (1951).

[71] R. E. Smalley, L. Wharton and D. H. Levy, Accounts of Chemical Research 10, 139 (1977).

[72] D. H. Levy, Science, 263 (1981).

[73] J. Bucher, D. Douglass, P. Xia and L. Bloomfield, Rev. Sci. Instrum. 61, 2374 (1990).

[74] N. Abuaf, J. Anderson, R. Andres, J. Fenn and D. Marsden, Science 155, 997 (1967).

[75] J. Anderson, R. Andres and J. Fenn, Molecular beams. Ross J.(ed.), 275 (1966).

[76] J. Anderson and J. Fenn, The physics of fluids 8, 780 (1965).

[77] B. Friedrich and D. Herschbach, Physics Today 56, 53 (2003).

[78] W. Gerlach and O. Stern, Zeitschrift für Physik 9, 349 (1922).

[79] G. Herzberg, Proceedings of the Royal Society of London. Series A. Mathematical and Physical Sciences 262, 291 (1961).

[80] J. Solomon, J. Chem. Phys. 47, 889 (1967).

[81] J. Solomon, C. Jonah, P. Chandra and R. Bersohn, J. Chem. Phys. 55, 1908 (1971). 
[82] M. N. Ashfold, N. H. Nahler, A. J. Orr-Ewing, O. P. Vieuxmaire, R. L. Toomes, T. N. Kitsopoulos, I. A. Garcia, D. A. Chestakov, S.-M. Wu and D. H. Parker, Phys. Chem. Chem. Phys. 8, 26 (2006).

[83] G. E. Busch, J. F. Cornelius, R. T. Mahoney, R. I. Morse, D. W. Schlosser and K. R. Wilson, Rev. Sci. Instrum. 41, 1066 (1970).

[84] G. E. Busch and K. R. Wilson, J. Chem. Phys. 56, 3626 (1972).

[85] G. E. Busch and K. R. Wilson, J. Chem. Phys. 56, 3638 (1972).

[86] G. E. Busch and K. R. Wilson, J. Chem. Phys. 56, 3655 (1972).

[87] J. Hepburn, R. Buss, L. Butler and Y. Lee, J. Phys. Chem. 87, 3638 (1983).

[88] E. J. Hintsa, X. Zhao and Y. T. Lee, J. Chem. Phys. 92, 2280 (1990).

[89] A. Yokoyama, X. Zhao, E. Hintsa, R. Continetti and Y. T. Lee, J. Chem. Phys. 92, 4222 (1990).

[90] R. Vasudev, R. Zare and R. Dixon, J. Chem. Phys. 80, 4863 (1984).

[91] R. Vasudev, R. Zare and R. Dixon, Chem. Phys. Lett. 96, 399 (1983).

[92] D. Eres, M. Gurnick and J. McDonald, J. Chem. Phys. 81, 5552 (1984).

[93] D. De Bruijn and J. Los, Rev. Sci. Instrum. 53, 1020 (1982).

[94] D. De Bruijn, J. Neuteboom and J. Los, Chem. Phys. 85, 233 (1984).

[95] D.-P. de Bruijn, J. Neuteboom, V. Sidis and J. Los, Chem. Phys. 85, 215 (1984).

[96] H. Helm, D. De Bruijn and J. Los, Physical review letters 53, 1642 (1984).

[97] W. Van der Zande, D. De Bruijn, J. Los, P. Kistemaker and S. McLuckey, International journal of mass spectrometry and ion processes 67, 161 (1985).

[98] W. Van der Zande, W. Koot, J. Peterson and J. Los, Chem. Phys. Lett. 140, 175 (1987).

[99] D. W. Chandler and P. L. Houston, J. Chem. Phys. 87, 1445 (1987).

[100] D. W. Chandler, J. W. Thoman Jr, M. H. Janssen and D. H. Parker, Chem. Phys. Lett. 156, 151 (1989).

[101] A. e. Eppink, T. J. B. and D. H. Parker, Rev. Sci. Instrum. 68, 3477 (1997).

[102] C. R. Gebhardt, T. P. Rakitzis, P. C. Samartzis, V. Ladopoulos and T. N. Kitsopoulos, Rev. Sci. Instrum. 72, 3848 (2001). 
[103] D. Townsend, M. P. Minitti and A. G. Suits, Rev. Sci. Instrum. 74, 2530 (2003).

[104] T. P. Rakitzis and R. N. Zare, J. Chem. Phys. 110, 3341 (1999).

[105] R. N. Zare and D. R. Herschbach, Proceedings of the IEEE 51, 173 (1963).

[106] G. Loge, J. Tiee and F. Wampler, J. Chem. Phys. 79, 196 (1983).

[107] D. S. Green, G. A. Bickel and S. C. Wallace, J. Mol. Spectrosc. 150, 303 (1991).

[108] D. S. Green and S. C. Wallace, J. Chem. Phys. 96, 5857 (1992).

[109] D. H. Parker, R. F. Delmdahl, B. B. Bakker and H. P. Loock, Journal of the Chinese Chemical Society 48, 327 (2001).

[110] R. Toomes, P. Samartzis, T. Rakitzis and T. Kitsopoulos, Chem. Phys. 301, 209 (2004).

[111] J. O. F. Thompson, C. Amarasinghe, C. D. Foley, N. Rombes, Z. Gao, S. N. Vogels, S. Y. T. van de Meerakker and A. G. Suits, J. Chem. Phys. 147, 074201 (2017).

[112] J. O. F. Thompson, C. Amarasinghe, C. D. Foley and A. G. Suits, J. Chem. Phys. 147, 013913 (2017).

[113] Y. Sato, Y. Matsumi, M. Kawasaki, K. Tsukiyama and R. Bersohn, J. Phys. Chem. 99, 16307 (1995).

[114] N. Sivakumar, G. Hall, P. Houston, J. Hepburn and I. Burak, J. Chem. Phys. 88, 3692 (1988).

[115] D. B. Kokh, H.-P. Liebermann and R. J. Buenker, J. Chem. Phys. 132, 074707 (2010).

[116] A. Kalume, L. George, P. Z. El-Khoury, A. N. Tarnovsky and S. A. Reid, J. Phys. Chem. A 114, 9919 (2010).

[117] G. Karras, S. Danakas and C. Kosmidis, J. Phys. Chem. A 115, 4186 (2011).

[118] C. P. Anderson, K. G. Spears, K. R. Wilson and R. J. Sension, J. Chem. Phys. 139, 194307 (2013).

[119] Habartovl'a, Alena, A. Obisesan, B. Minofar and Roeselovl'a, Martina, Theor. Chem. Acc. 133, 1455 (2014).

[120] A. R. Attar, A. Bhattacherjee and S. R. Leone, J. Phys. Chem. Lett. 6, 5072 (2015).

[121] W. G. Merrill, F. F. Crim and A. S. Case, Phys. Chem. Chem. Phys. 18, 32999 (2016).

[122] G. Baum, P. Felder and J. R. Huber, J. Chem. Phys. 98, 1999 (1993).

[123] G. Baum and J. R. Huber, Chem. Phys. Lett. 203, 261 (1993). 
[124] G. Baum and J. R. Huber, Chem. Phys. Lett. 213, 427 (1993).

[125] P. Felder and C. Demuth, Chem. Phys. Lett. 208, 21 (1993).

[126] F. S. Rowland, C. C. Chou and P. Angelberger, J. Phys. Chem. 75, 2536 (1971).

[127] J. J. Lin, Y. Chen, Y. Y. Lee, Y. T. Lee and X. Yang, Chem. Phys. Lett. 361, 374 (2002).

[128] A. Shastri, P. J. Singh, S. Krishnakumar, A. K. Das and B. N. R. Sekhar, Phys. Chem. Chem. Phys. 19, 6454 (2017).

[129] K. Suto, Y. Sato, C. L. Reed, V. Skorokhodov, Y. Matsumi and M. Kawasaki, J. Phys. Chem. A 101, 1222 (1997).

[130] P. Zou, W. S. McGivern, O. Sorkhabi, A. G. Suits and S. W. North, J. Chem. Phys. 113, 7149 (2000).

[131] G.-J. Wang, R.-S. Zhu, H. Zhang, K.-L. Han, G.-Z. He and N.-Q. Lou, Chem. Phys. Lett. 288, 429 (1998).

[132] Y. Liu and L. J. Butler, J. Chem. Phys. 121, 11016 (2004).

[133] P. W. Browning, D. C. Kitchen, M. F. Arendt and L. J. Butler, J. Phys. Chem. 100, 7765 (1996).

[134] M. L. Morton, L. J. Butler, T. A. Stephenson and F. Qi, J. Chem. Phys. 116, 2763 (2002).

[135] M. Kawasaki, K. Kasatani, H. Sato, H. Shinohara and N. Nishi, Chem. Phys. 88, 135 (1984).

[136] Y.-R. Lee and S.-M. Lin, J. Chem. Phys. 108, 134 (1998).

[137] L. R. McCunn, D. I. G. Bennett, L. J. Butler, H. Fan, F. Aguirre and S. T. Pratt, J. Phys. Chem. A 110, 843 (2006).

[138] S. Harper, P. Calandra and S. D. Price, Phys. Chem. Chem. Phys. 3, 741 (2001).

[139] J. C. Robinson, N. E. Sveum and D. M. Neumark, J. Chem. Phys. 119, 5311 (2003).

[140] H. Fan and S. Pratt, J. Chem. Phys. 124, 144313 (2006).

[141] H. Shen, L. Hua, Z. Cao, C. Hu and B. Zhang, Opt. Commun. 282, 387 (2009).

[142] L. J. Radziemski and V. Kaufman, J. Opt. Soc. Am. 59, 424 (1969).

[143] A. Kramida, Yu. Ralchenko, J. Reader and N. A. Team, (\{NIST Atomic Spectra Database (ver. 5.5.6), [Online]. Available: $\{\backslash \mathrm{tt}\{\mathrm{https://physics.nist.gov/asd}\}$ \} [2017, April 9]. National Institute of Standards and Technology, Gaithersburg, MD.\}, 2018). 
[144] T. H. Dunning Jr, J. Chem. Phys. 90, 1007 (1989).

[145] T. H. Dunning Jr, K. A. Peterson and A. K. Wilson, J. Chem. Phys. 114, 9244 (2001).

[146] D. E. Woon and T. H. Dunning Jr, J. Chem. Phys. 98, 1358 (1993).

[147] P. J. Knowles and H.-J. Werner, Chem. Phys. Lett. 115, 259 (1985).

[148] H.-J. Werner and P. J. Knowles, J. Chem. Phys. 82, 5053 (1985).

[149] T. Shiozaki, Gy\Ho, rffy, Werner, P. Celani and H.-J. Werner, J. Chem. Phys. 135, 081106 (2011).

[150] T. Shiozaki, G. Knizia and H.-J. Werner, J. Chem. Phys. 134, 034113 (2011).

[151] W. Eisfeld, J. Phys. Chem. A 110, 3903 (2006).

[152] 1. M\o, Chr and M. S. Plesset, Phys. Rev. 46, 618 (1934).

[153] H.-J. Werner, P. J. Knowles, G. Knizia, F. R. Manby and t. Sch $\ " u$, Martin, WIRES Comput. Mol. Sci. 2, 242 (2012).

[154] A. Fahr, P. Hassanzadeh, B. Laszlo and R. E. Huie, Chem. Phys. 215, 59 (1997).

[155] J. L. Holmes and F. P. Lossing, Can. J. Chem. 57, 249 (1979).

[156] A. Burcat and B. Ruscic, Third millenium ideal gas and condensed phase thermochemical database for combustion with updates from active thermochemical tables. (Argonne National Laboratory Argonne, IL, 2005).

[157] A. J. Beaulieu, Appl. Phys. Lett. 16, 504 (1970).

[158] O. R. Wood, R. L. Abrams and T. J. Bridges, Appl. Phys. Lett. 17, 376 (1970).

[159] J. A. Beaulieu, P. IEEE 59, 667 (1971).

[160] R. L. Abrams and O. R. Wood, Appl. Phys. Lett. 19, 518 (1971).

[161] Y.-L. Pan, A. F. Bernhardt and J. R. Simpson, Rev. Sci. Instrum. 43, 662 (1972).

[162] F. M. Lussier and J. I. Steinfeld, Chem. Phys. Lett. 50, 175 (1977).

[163] P. A. Schulz, A. S. Sudbcent, D. J. Krajnovich, H. S. Kwok, Y. R. Shen and Y. T. Lee, Ann. Rev. Phys. Chem. 30, 379 (1979).

[164] E. J. Hintsa, A. M. Wodtke and Y. T. Lee, J. Phys. Chem. 92, 5379 (1988).

[165] A. M. Wodtke, E. J. Hintsa and Y. T. Lee, J. Phys. Chem. 90, 3549 (1986). 
[166] G. Hancock and K. G. McKendrick, J. Phys. Chem. 92, 1839 (1988).

[167] A. S. Sudbo/, P. A. Schulz, E. R. Grant, Y. R. Shen and Y.-T. Lee, J. Chem. Phys. 70, 912 (1979).

[168] A. M. Wodtke, E. J. Hintsa and Y. T. Lee, J. Chem. Phys. 84, 1044 (1986).

[169] N. Herath and A. G. Suits, Ann. Rev. Phys. Chem. 2, 642 (2011).

[170] J. M. Bowman and B. C. Shepler, Ann. Rev. Phys. Chem. 62, 531 (2011).

[171] E. R. Grant, M. J. Coggiola, Y. T. Lee, P. A. Schulz, A. S. Sudbo and Y. R. Shen, Chem. Phys. Lett. 52, 595 (1977).

[172] A. S. Sudbo/, P. A. Schulz, Y. R. Shen and Y. T. Lee, J. Chem. Phys. 69, 2312 (1978).

[173] F. Huisken, D. Krajnovich, Z. Zhang, Y. R. Shen and Y.-T. Lee, J. Chem. Phys. 78, 3806 (1983).

[174] X. Zhao, E. J. Hintsa and Y. T. Lee, J. Chem. Phys. 88, 801 (1988).

[175] C. A. Longfellow and Y. T. Lee, J. Phys. Chem. 99, 15532 (1995).

[176] A. Dey, R. Fernando, C. Abeysekera, Z. Homayoon, J. M. Bowman and A. G. Suits, J. Chem. Phys. 140, 054305 (2014).

[177] R. Fernando, N. M. Ariyasingha and A. G. Suits, Chem. Phys. Lett. 645, 76 (2016).

[178] R. Fernando, C. Qu, J. M. Bowman, R. W. Field and A. G. Suits, J. Phys. Chem. Lett. 6, 2457 (2015).

[179] T. Shimanouchi, Tables of molecular vibrational frequencies. (US Government Printing Office, 1973).

[180] C. Abeysekera, L. N. Zack, G. B. Park, B. Joalland, J. M. Oldham, K. Prozument, N. M. Ariyasingha, I. R. Sims, R. W. Field and A. G. Suits, J. Chem. Phys. 141, 214203 (2014).

[181] J. M. Oldham, C. Abeysekera, B. Joalland, L. N. Zack, K. Prozument, I. R. Sims, G. B. Park, R. W. Field and A. G. Suits, J. Chem. Phys. 141, 154202 (2014).

[182] K. Prozument, G. B. Park, R. G. Shaver, A. K. Vasiliou, J. M. Oldham, D. E. David, J. S. Muenter, J. F. Stanton, A. G. Suits and G. B. Ellison, Phys. Chem. Chem. Phys. 16, 15739 (2014).

[183] B. M. Broderick, N. Suas-David, N. Dias and A. G. Suits, Phys. Chem. Chem. Phys. 20, 5517 (2018).

[184] J.-D. Chai and M. Head-Gordon, Phys. Chem. Chem. Phys. 10, 6615 (2008). 
[185] J. A. Montgomery Jr, M. J. Frisch, J. W. Ochterski and G. A. Petersson, J. Chem. Phys. 110, 2822 (1999).

[186] J. W. Ochterski, G. A. Petersson and J. A. Montgomery Jr, J. Chem. Phys. 104, 2598 (1996).

[187] G. Petersson and M. A. Al-Laham, J. Chem. Phys. 94, 6081 (1991).

[188] M. J. Frisch, G. W. Trucks, H. B. Schlegel, G. E. Scuseria, M. A. Robb, J. R. Cheeseman, G. Scalmani, V. Barone, G. A. Petersson, H. Nakatsuji, X. Li, M. Caricato, A. V. Marenich, J. Bloino, B. G. Janesko, R. Gomperts, B. Mennucci, H. P. Hratchian, J. V. Ortiz, A. F. Izmaylov, J. L. Sonnenberg, Williams, F. Ding, F. Lipparini, F. Egidi, J. Goings, B. Peng, A. Petrone, T. Henderson, D. Ranasinghe, V. G. Zakrzewski, J. Gao, N. Rega, G. Zheng, W. Liang, M. Hada, M. Ehara, K. Toyota, R. Fukuda, J. Hasegawa, M. Ishida, T. Nakajima, Y. Honda, O. Kitao, H. Nakai, T. Vreven, K. Throssell, J. A. Montgomery Jr., J. E. Peralta, F. Ogliaro, M. J. Bearpark, J. J. Heyd, E. N. Brothers, K. N. Kudin, V. N. Staroverov, T. A. Keith, R. Kobayashi, J. Normand, K. Raghavachari, A. P. Rendell, J. C. Burant, S. S. Iyengar, J. Tomasi, M. Cossi, J. M. Millam, M. Klene, C. Adamo, R. Cammi, J. W. Ochterski, R. L. Martin, K. Morokuma, O. Farkas, J. B. Foresman and D. J. Fox, (Wallingford, CT, 2016).

[189] C. Western, University of Bristol 7, 108 (2010).

[190] B. Joalland, Y. Shi, A. Kamasah, A. G. Suits and A. M. Mebel, Nat. Commun. 5, 4064 (2014).

[191] B. Joalland, R. Van Camp, Y. Shi, N. Patel and A. G. Suits, J. Phys. Chem. A 117, 7589 (2013).

[192] W. H. Miller, J. Am. Chem. Soc. 105, 216 (1983).

[193] W. H. Miller, R. Hernandez, C. B. Moore and W. F. Polik, J. Chem. Phys. 93, 5657 (1990).

[194] W. F. Polik, D. R. Guyer, W. H. Miller and C. B. Moore, J. Chem. Phys. 92, 3471 (1990).

[195] S. A. Lahankar, S. D. Chambreau, D. Townsend, F. Suits, J. Farnum, X. Zhang, J. M. Bowman and A. G. Suits, J. Chem. Phys. 125, 044303 (2006).

[196] J. M. Bowman and X. Zhang, Phys. Chem. Chem. Phys. 8, 321 (2006).

[197] D. Clouthier and D. Ramsay, Ann. Rev. Phys. Chem. 34, 31 (1983).

[198] G. Herzberg, Molecular Spectra and Molecular Structure. Vol. 3: Electronic Spectra and Electronic Structure of Polyatomic Molecules. (1966).

[199] G. Dieke and G. Kistiakowsky, Phys. Rev. 45, 4 (1934).

[200] D. Moule and A. Walsh, Chem. Rev. 75, 67 (1975).

[201] C. B. Moore and J. C. Weisshaar, Ann. Rev. Phys. Chem. 34, 525 (1983). 
[202] P. L. Houston, X. Wang, A. Ghosh, J. M. Bowman, M. S. Quinn and S. H. Kable, J. Chem. Phys. 147, 013936 (2017).

[203] T. J. Butenhoff, K. L. Carleton, R. D. van Zee and C. B. Moore, J. Chem. Phys. 94, 1947 (1991).

[204] R. D. van Zee, C. D. Pibel, T. J. Butenhoff and C. B. Moore, J. Chem. Phys. 97, 3235 (1992).

[205] T. J. Butenhoff, K. L. Carleton, M.-C. Chuang and C. B. Moore, J. Chem. Soc. Farad. T. 2 85, 1155 (1989).

[206] S. Rashev and D. Moule, Open Chem. 11, 1 (2013).

[207] S. Rashev and D. C. Moule, J. Mol. Spectrosc. 286, 21 (2013).

[208] R. Bocquet, J. Demaison, J. Cosléou, A. Friedrich, L. Margules, S. Macholl, H. Mäder, M. Beaky and G. Winnewisser, J. Mol. Spectrosc. 195, 345 (1999).

[209] M. Allegrini, J. Johns and A. McKellar, Can. J. Phys. 56, 859 (1978).

[210] J. Johns and A. McKellar, J. Mol. Spectrosc. 64, 327 (1977).

[211] D. Davidson, B. Stoicheff and H. Bernstein, J. Chem. Phys. 22, 289 (1954).

[212] K. K. Ellsworth, B. D. Lajiness, J. P. Lajiness and W. F. Polik, J. Mol. Spectrosc. 252, 205 (2008).

[213] O. Zakharenko, R. A. Motiyenko, L. Margulès and T. R. Huet, J. Mol. Spectrosc. 317, 41 (2015).

[214] B. J. Orr and G. F. Nutt, J. Mol. Spectrosc. 84, 272 (1980).

[215] V. Goncharov, S. A. Lahankar, J. D. Farnum, J. M. Bowman and A. G. Suits, J. Phys. Chem. A 113, 15315 (2009).

[216] V. Job, V. Sethuraman and K. Innes, J. Mol. Spectrosc. 30, 365 (1969).

[217] V. Sethuraman, V. Job and K. Innes, J. Mol. Spectrosc. 33, 189 (1970).

[218] D. J. Bamford, S. V. Filseth, M. F. Foltz, J. W. Hepburn and C. B. Moore, J. Chem. Phys. 82, 3032 (1985).

[219] R. G. Miller and E. K. Lee, J. Chem. Phys. 68, 4448 (1978).

[220] M. Kang, M. Choi, A. M. Stutz and K. i. Tatematsu, The Astrophysical Journal 814, 31 (2015).

[221] R. B. Loren and A. Wootten, The Astrophysical Journal 299, 947 (1985). 
[222] S. Zahorecz, I. Jimenez-Serra, L. Testi, K. Immer, F. Fontani, P. Caselli, K. Wang and L. Toth, Astron. Astrophys. 602, L3 (2017).

[223] F. Fontani, G. Busquet, A. Palau, P. Caselli, Á. Sánchez-Monge, J. Tan and M. Audard, Astron. Astrophys. 575, A87 (2015).

[224] C. Codella, C. Ceccarelli, B. Lefloch, F. Fontani, G. Busquet, P. Caselli, C. Kahane, D. Lis, V. Taquet and M. Vasta, The Astrophysical Journal Letters 757, L9 (2012).

[225] L. Gomez, A. Perrin and G. Mellau, J. Mol. Spectrosc. 256, 28 (2009).

[226] D. Clouthier, A. Craig and D. Ramsay, Can. J. Phys. 61, 1073 (1983).

[227] D. Clouthier, A. Craig and F. Birss, Can. J. Phys. 62, 973 (1984).

[228] D. E. Reisner, R. W. Field, J. L. Kinsey and H. L. Dai, J. Chem. Phys. 80, 5968 (1984).

[229] R. G. Miller and E. K. Lee, Chem. Phys. Lett. 41, 52 (1976).

[230] L. T. Molina, K. Y. Tang, J. R. Sodeau and E. K. Lee, J. Phys. Chem. 82, 2575 (1978).

[231] R. Schinke and V. Engel, Faraday Discuss. 82, 111 (1986).

[232] M. C. Chuang, M. F. Foltz and C. B. Moore, J. Chem. Phys. 87, 3855 (1987).

[233] A. C. Terentis, S. E. Waugh, G. F. Metha and S. H. Kable, J. Chem. Phys. 108, 3187 (1998).

[234] J. G. Ouzounian and F. A. Anet, J. Labelled Compd. Rad. 23, 401 (1986).

[235] G. C. Bjorklund, C. Ausschnitt, R. R. Freeman and R. H. Storz, Applied Physics Letters 33, 54 (1978).

[236] G. A. Bickel and G. A. McRae, Spectrochimica Acta Part B: Atomic Spectroscopy 55, 57 (2000).

[237] J. Brown and D. Ramsay, Can. J. Phys. 53, 2232 (1975).

[238] D. E. Milligan and M. E. Jacox, J. Chem. Phys. 51, 277 (1969).

[239] G. Herzberg and D. A. Ramsay, Proceedings of the Royal Society of London. Series A. Mathematical and Physical Sciences 233, 34 (1955).

[240] J. Johns, S. Priddle and D. Ramsay, Discussions of the Faraday Society 35, 90 (1963).

[241] C. Stöck, X. Li, H.-M. Keller, R. Schinke and F. Temps, J. Chem. Phys. 106, 5333 (1997).

[242] G. Rumbles, E. K. Lee and J. J. Valentini, J. Chem. Soc. Farad. T. 86, 3837 (1990).

[243] H.-M. Yin, K. Nauta and S. H. Kable, J. Chem. Phys. 122, 194312 (2005). 
[244] K. L. K. Lee, M. S. Quinn, S. J. Kolmann, S. H. Kable and M. J. Jordan, J. Chem. Phys. 148, 194113 (2018).

[245] Y. Guo, D. L. Thompson and T. D. Sewell, J. Chem. Phys. 104, 576 (1996).

[246] W. H. Miller, W. L. Hase and C. L. Darling, J. Chem. Phys. 91, 2863 (1989).

[247] W. Scott Hopkins, H.-P. Loock, B. Cronin, M. G. Nix, A. L. Devine, R. N. Dixon and M. N. Ashfold, J. Chem. Phys. 127, 064301 (2007).

[248] M. S. Quinn, K. Nauta, M. J. Jordan, J. M. Bowman, P. L. Houston and S. H. Kable, Science 369, 1592 (2020).

[249] B. Schramm, D. Bamford and C. B. Moore, Chem. Phys. Lett. 98, 305 (1983).

[250] M. S. Quinn, K. Nauta and S. H. Kable, Molecular Physics, e1836412 (2020).

[251] B. Fu, B. C. Shepler and J. M. Bowman, J. Am. Chem. Soc. 133, 7957 (2011).

[252] J. D. Farnum, X. Zhang and J. M. Bowman, J. Chem. Phys. 126, 134305 (2007).

[253] B. C. Shepler, E. Epifanovsky, P. Zhang, J. M. Bowman, A. I. Krylov and K. Morokuma, J. Phys. Chem. A 112, 13267 (2008).

[254] W. F. Polik, D. R. Guyer and C. B. Moore, J. Chem. Phys. 92, 3453 (1990).

[255] H. Dai, C. Korpa, J. Kinsey and R. Field, J. Chem. Phys. 82, 1688 (1985).

[256] D. J. Nesbitt and R. W. Field, J. Phys. Chem. 100, 12735 (1996).

[257] T. Uzer and W. Miller, Physics reports 199, 73 (1991).

[258] D. W. Chandler, J. Chem. Phys. 132, 110901 (2010).

[259] C. M. Lovejoy and D. J. Nesbitt, J. Chem. Phys. 93, 5387 (1990).

[260] B. C. Shepler, Y. Han and J. M. Bowman, J. Phys. Chem. Lett. 2, 834 (2011).

[261] T. J. Butenhoff, K. L. Carleton and C. B. Moore, J. Chem. Phys. 92, 377 (1990).

[262] T. Tanaka and Y. Morino, J. Mol. Spectrosc. 33, 538 (1970).

[263] H. S. Müller, E. A. Cohen and D. Christen, J. Mol. Spectrosc. 216, 335 (2002).

[264] B. R. Heazlewood, M. J. Jordan, S. H. Kable, T. M. Selby, D. L. Osborn, B. C. Shepler, B. J. Braams and J. M. Bowman, Proceedings of the National Academy of Sciences 105, 12719 (2008). 
[265] P. Houston and S. Kable, Proceedings of the National Academy of Sciences 103, 16079 (2006).

[266] L. Rubio-Lago, G. Amaral, A. Arregui, J. Gonzalez-Vazquez and L. Banares, Phys. Chem. Chem. Phys. 14, 6067 (2012).

[267] C.-H. Yang, S. Bhattacharyya, L. Liu, W.-h. Fang and K. Liu, Chemical Science (2020). 


\section{VITA}

Casey D. Foley was born in Saginaw, MI and grew up in Bay City, MI. He obtained a B.S. in Biochemistry from Saginaw Valley State University in May 2013. His Ph.D. candidacy was obtained under Professor Sarah Trimpin at Wayne State University in Detroit, where he began studying in August 2013. Upon moving to Columbia, MO in May 2016 to finish his Ph.D. under Professor Arthur Suits at the University of Missouri, Casey began participating in a variety of chemical dynamics experiments. The bulk of his experience in the Suits Group has come from traditional pump-probe unimolecular photodissociation experiments. In the summer of 2018, he

characterized energetic nanoporous silicon at the Army Research Lab under the guidance of Philip Guerieri and Wayne Churaman. His Ph.D. in Physical Chemistry was completed in December 2020. 